\title{
Conformal supergravity in five dimensions: new approach and applications
}

\author{
Daniel Butter, ${ }^{a}$ Sergei M. Kuzenko, ${ }^{b}$ Joseph Novak $^{b}$ and \\ Gabriele Tartaglino-Mazzucchelli ${ }^{b}$ \\ ${ }^{a}$ Nikhef Theory Group, \\ Science Park 105, 1098 XG Amsterdam, The Netherlands \\ ${ }^{b}$ School of Physics M013, The University of Western Australia, \\ 35 Stirling Highway, Crawley W.A. 6009, Australia \\ E-mail: dbutter@nikhef.nl, sergei.kuzenko@uwa.edu.au, \\ joseph.novak@uwa.edu.au, gabriele.tartaglino-mazzucchelli@uwa.edu.au
}

ABstract: We develop a new off-shell formulation for five-dimensional (5D) conformal supergravity obtained by gauging the 5D superconformal algebra in superspace. An important property of the conformal superspace introduced is that it reduces to the superconformal tensor calculus (formulated in the early 2000's) upon gauging away a number of superfluous fields. On the other hand, a different gauge fixing reduces our formulation to the $\mathrm{SU}(2)$ superspace of arXiv:0802.3953, which is suitable to describe the most general off-shell supergravity-matter couplings. Using the conformal superspace approach, we show how to reproduce practically all off-shell constructions derived so far, including the supersymmetric extensions of $R^{2}$ terms, thus demonstrating the power of our formulation. Furthermore, we construct for the first time a supersymmetric completion of the Ricci tensor squared term using the standard Weyl multiplet coupled to an off-shell vector multiplet. In addition, we present several procedures to generate higher-order off-shell invariants in supergravity, including higher-derivative ones. The covariant projective multiplets proposed in arXiv:0802.3953 are lifted to conformal superspace, and a manifestly superconformal action principle is given. We also introduce unconstrained prepotentials for the vector multiplet, the $\mathcal{O}(2)$ multiplet (i.e., the linear multiplet without central charge) and $\mathcal{O}(4+n)$ multiplets, with $n=0,1, \ldots$ Superform formulations are given for the BF action and the non-abelian Chern-Simons action. Finally, we describe locally supersymmetric theories with gauged central charge in conformal superspace.

Keywords: Extended Supersymmetry, Superspaces, Chern-Simons Theories, Supergravity Models

ARXiv EPRINT: 1410.8682 


\section{Contents}

1 Introduction 1

2 Conformal superspace in five dimensions 4

2.1 The superconformal algebra 4

2.2 Gauging the superconformal algebra 5

2.3 Conformal supergravity 8

$\begin{array}{lll}2.4 & \text { Full superspace actions } & 11\end{array}$

\begin{tabular}{ll}
2.5 & Gravitational composite $\mathcal{O}(2)$ multiplet \\
\hline
\end{tabular}

2.6 Turning on the Yang-Mills multiplet 12

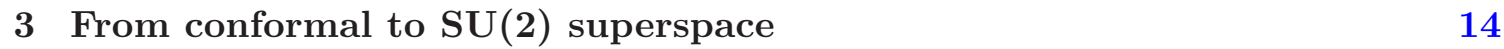

$\begin{array}{lll}3.1 & \text { Degauging to } \mathrm{SU}(2) \text { superspace } & 14\end{array}$

$\begin{array}{ll}3.2 \text { The degauged special conformal connection } & 15\end{array}$

$\begin{array}{lll}3.3 & \text { The conformal origin of the super Weyl transformations } & 18\end{array}$

4 The Weyl multiplet $\quad 19$

4.1 Component fields and curvatures from superspace 20

4.2 Analysis of the curvature constraints 22

4.3 Supersymmetry transformations of the fundamental fields 24

4.4 A new choice for component constraints 24

5 The covariant projective multiplets in conformal superspace $\quad 27$

$\begin{array}{ll}5.1 \text { Covariant projective multiplets } & 28\end{array}$

5.2 Analytic projection operator 31

$\begin{array}{ll}5.3 & \text { The action principle } \\ \end{array}$

6 Prepotentials for the vector multiplet $\quad 35$

$\begin{array}{lll}6.1 & \text { Tropical prepotential } & 35\end{array}$

$\begin{array}{lll}6.2 & \text { Polar hypermultiplets } & 36\end{array}$

6.3 Arctic and antarctic representations 37

6.4 Abelian field strength: contour integral representation 39

$\begin{array}{lll}6.5 & \text { Mezincescu's prepotential } & 40\end{array}$

$\begin{array}{lll}6.6 & \text { Composite } \mathcal{O}(2) \text { multiplet } & 40\end{array}$

$\begin{array}{lll}7 & \text { The } \mathcal{O}(2) & \text { multiplet in conformal superspace }\end{array}$

7.1 Prepotential formulation for the $\mathcal{O}(2)$ multiplet 42

$\begin{array}{lll}7.2 & \text { Composite vector multiplet } & 43\end{array}$

$\begin{array}{lll}7.3 & \text { BF coupling } & 43\end{array}$

7.4 Gauge invariance 44

7.5 Universality of the BF action 44

$\begin{array}{lll}7.6 & \text { Full superspace invariants } & 45\end{array}$ 
8 Superform formulation for the BF action 46

8.1 Superform geometry of the $\mathcal{O}(2)$ multiplet 46

$\begin{array}{lll}8.2 & \text { Superform action for the } \mathcal{O}(2) \text { multiplet } & 47\end{array}$

$\begin{array}{lll}\text { 8.2.1 General setup } & 47\end{array}$

8.2.2 Superform action for the $\mathcal{O}(2)$ multiplet 48

8.2.3 Component BF action 49

9 Abelian Chern-Simons theory $\quad 50$

10 Non-abelian Chern-Simons theory

10.1 The Chern-Simons five-form 54

10.2 The curvature-induced five-form $\quad 54$

10.3 The non-abelian Chern-Simons action $\quad 57$

11 Supermultiplets with gauged central charge $\quad 58$

$\begin{array}{ll}\text { 11.1 Gauging a central charge in conformal superspace } & 59\end{array}$

$\begin{array}{ll}\text { 11.2 The linear multiplet with central charge } & 59\end{array}$

11.2.1 Superform formulation for the linear multiplet $\quad 60$

$\begin{array}{ll}\text { 11.2.2 Action principle } & 62\end{array}$

11.3 Gauge two-form multiplet $\quad 62$

$\begin{array}{ll}11.4 \text { Large tensor multiplet } & 64\end{array}$

12 Off-shell (gauged) supergravity $\quad 66$

$\begin{array}{ll}12.1 \text { Superspace formulation } & 66\end{array}$

$\begin{array}{ll}12.2 \text { Component formulation } & 69\end{array}$

13 Dilaton Weyl multiplets and superforms $\quad 70$

$\begin{array}{ll}13.1 \text { The dilaton Weyl multiplet } & 71\end{array}$

13.2 The deformed dilaton Weyl multiplet $\quad 73$

13.3 The deformed dilaton Weyl multiplet with Chern-Simons couplings $\quad 74$

14 Higher derivative couplings $\quad \mathbf{7 5}$

$\begin{array}{ll}\text { 14.1 Composite primary multiplets and invariants } & 75\end{array}$

$\begin{array}{ll}14.2 \text { Composite vector multiplets } & 77\end{array}$

$\begin{array}{ll}14.3 \text { Ricci squared } \mathcal{O}(2) \text { multiplet } & 79\end{array}$

14.4 Supersymmetric $R^{2}$ invariants $\quad 81$

14.5 The supersymmetric Ricci squared term and the dilaton Weyl multiplet 82

15 Concluding remarks $\quad 84$

$\begin{array}{lr}\text { A Notation and conventions } & 87\end{array}$

B The conformal Killing supervector fields of $\mathbb{R}^{5 \mid 8} \quad 90$ 
$\begin{array}{ll}\text { D Conventions for 5D conformal supergravity } & 94\end{array}$

E The $\mathcal{O}(2)$ multiplet prepotential from harmonic superspace $\quad 96$

$\begin{array}{lll}\text { F } & \text { Gauge freedom for the } \mathcal{O}(2) \text { multiplet } & 97\end{array}$

G Prepotentials for $\mathcal{O}(4+n)$ multiplets, $n=0,1, \ldots$, from harmonic superspace

\section{Introduction}

Minimal supergravity in five spacetime dimensions ${ }^{1}(5 \mathrm{D})$ was introduced more than three decades ago by Cremmer [4] and independently by Chamseddine and Nicolai [5]. A year later, an off-shell formulation for this theory was sketched by Howe [6] (building on the supercurrent multiplet constructed by him and Lindström [7]), who used superspace techniques and provided a $5 \mathrm{D}$ extension of the so-called $\mathcal{N}=2$ minimal supergravity multiplet in four dimensions $[8,9]$. Since then, 5D minimal supergravity and its matter couplings have extensively been studied at the component level, both in on-shell [10-13] and off-shell [1423 ] settings. The superspace approach to general off-shell 5D $\mathcal{N}=1$ supergravity-matter systems has been developed in $[24-26]^{2}$

Off-shell formulations for supergravity make the supersymmetry transformation laws of fields model-independent and, in principle, offer a tensor calculus to generate arbitrary supergravity-matter couplings. A non-conformal tensor calculus for $5 \mathrm{D} \mathcal{N}=1$ supergravity was developed by Zucker $[14,15]$ (see also [16] for a review and applications). By making use of Howe's minimal supergravity multiplet [6] and the supercurrent multiplet [7] (both carefully reduced to components), he extended to five dimensions various off-shell techniques developed for $4 \mathrm{D} \mathcal{N}=2$ matter-coupled supergravity (see, e.g., [28] for a review). A more complete approach is the 5D superconformal tensor calculus developed independently by two groups: Fujita, Kugo, and Ohashi ${ }^{3}$ [17-20] and Bergshoeff et al. [21-23]. Among the most interesting off-shell constructions obtained by applying the 5D superconformal calculus are (i) the non-abelian Chern-Simons action coupled to conformal supergravity [18], (ii) the massive tensor multiplet models [20], and (iii) the supersymmetric completions of $R^{2}$ terms [29-32].

\footnotetext{
${ }^{1}$ Historically, different authors use different notations, $\mathcal{N}=1$ or $\mathcal{N}=2$, for $5 \mathrm{D}$ supersymmetric theories with eight supercharges. We choose to use $\mathcal{N}=1$ following, e.g., [1-3].

${ }^{2}$ Refs. [24, 25] made use of Howe's minimal supergravity multiplet [6]. Ref. [26] developed a superspace formulation for conformal supergravity, which in this paper will be referred to as $\mathrm{SU}(2)$ superspace. In five dimensions, there is only one superconformal algebra, $\mathrm{F}^{2}(4)$ [27], and it corresponds to the choice $\mathcal{N}=1$. This is why one can simply speak of $5 \mathrm{D}$ conformal supergravity.

${ }^{3}$ Actually refs. $[17,18]$ presented the $5 \mathrm{D}$ tensor calculus in which some of the superconformal symmetries $(S$ and $K)$ are gauge fixed.
} 
Within the component approaches of [14-23], hypermultiplets are either on-shell or involve a gauged central charge. As is well known, such hypermultiplet realizations cannot be used to provide an off-shell formulation for the most general locally supersymmetric sigma model. It is also known that such a sigma model formulation, if it exists, requires the use of off-shell hypermultiplets possessing an infinite number of auxiliary fields. The latter feature of the off-shell hypermultiplets makes them extremely difficult to work with at the component level. This problem was solved within the superspace approach to $5 \mathrm{D} \mathcal{N}=1$ supergravity-matter systems [24-26] by putting forward the novel concept of covariant projective multiplets. These supermultiplets are a curved-superspace extension of the 4D $\mathcal{N}=2$ and $5 \mathrm{D} \mathcal{N}=1$ superconformal projective multiplets [33, 34]. The latter reduce to the off-shell projective multiplets pioneered by Lindström and Roček [35-37] in the 4D $\mathcal{N}=2$ super-Poincaré case and generalized to the cases of $5 \mathrm{D} \mathcal{N}=1$ Poincaré and anti-de Sitter supersymmetries in [2] and [3], respectively. Among the most interesting covariant projective multiplets are polar ones that have infinitely many auxiliary fields and indeed are suitable to realize the most general locally supersymmetric sigma model. These have never appeared within the component settings of [14-23].

This paper is devoted to new applications of the superspace approach to $5 \mathrm{D} \mathcal{N}=1$ matter-coupled supergravity [24-26]. In order to make a better transition to the superconformal calculus of [17-23], we present an extension of the superspace formulation for 5D conformal supergravity given in [26]. Such an extension is based on the concept of conformal superspace [38-40].

Conformal superspace is an off-shell formulation for conformal supergravity based on gauging the superconformal algebra in superspace. It was originally developed for $\mathcal{N}=1$ and $\mathcal{N}=2$ supergravity theories in four dimensions [38, 39] and more recently for $\mathcal{N}$ extended conformal supergravity in three dimensions [40]. ${ }^{4}$ For example, one may think of the $4 \mathrm{D} \mathcal{N}=1$ or $\mathcal{N}=2$ conformal superspace as a superspace analogue of the corresponding superconformal multiplet calculus developed many years earlier in the component setting, see e.g. [28] for a pedagogical review, since both approaches are gauge theories of the superconformal group. From a technical point of view, conformal superspace is a more general setting, since the gauge superfields contain more component fields and the gauge group is much larger than in the superconformal calculus. However, it turns out that the former formulation reduces to the latter upon gauging away a number of superfluous component fields. On the other hand, a different gauge fixing allows one to reduce conformal superspace to more traditional superspace settings. For instance, in the $4 \mathrm{D} \mathcal{N}=2$ case a certain gauge fixing reduces the conformal superspace of [39] to the so-called $\mathrm{U}(2)$ superspace [46], which has been used to construct the most general off-shell supergravity-matter couplings [47]. Thus conformal superspace provides a bridge between the component superconformal calculus and more traditional superspace formulations for conformal supergravity.

\footnotetext{
${ }^{4}$ In the physics literature, the name "conformal space" has been used since the 1930s. It was Dirac [41] who, following Veblen [42], introduced it for the conformal compactification of 4D Minkowski space, on which the conformal group acts transitively. Since the 1980s, the name "conformal superspace" has also been used for supersymmetric extensions of this construction [43, 44] (see also [33, 45] for more recent presentations). We hope no confusion may occur in our usage.
} 
Recent applications of the conformal superspace approach have involved constructing (i) the $\mathcal{N}$-extended conformal supergravity actions in three dimensions for $3 \leq \mathcal{N} \leq$ $6[48,49]$, and (ii) new higher-derivative invariants in $4 \mathrm{D} \mathcal{N}=2$ supergravity, including the Gauss-Bonnet term [50]. This paper is the first to explore applications of conformal superspace in five dimensions. In particular, we will demonstrate that the formalism of conformal superspace provides new tools to construct various composite primary multiplets that can be used to generate higher-order off-shell invariants in supergravity, including higher-derivative ones.

This paper is organized as follows. Section 2 describes the geometry of conformal superspace in five dimensions. In particular, we present the procedure in which the superconformal algebra is gauged in superspace and show how to constrain the resulting geometry to describe conformal supergravity, thus deriving a new off-shell formulation. We also describe the Yang-Mills multiplet in conformal superspace. In section 3 we show how the superspace formulation for conformal supergravity proposed in [26] may be viewed as a gauge-fixed version of conformal superspace. Section 4 is devoted to uncovering the component structure of conformal superspace and comparing it to the existing superconformal tensor calculus [19-23]. In section 5 we lift the covariant projective multiplets of [24-26] to conformal superspace. A general procedure to generate such multiplets is given. We also present a universal locally supersymmetric action principle. Section 6 presents prepotential formulations for the vector multiplet in conformal superspace. In section 7 we develop a prepotential formulation for the $\mathcal{O}(2)$ multiplet and discuss its universal role in generating actions. We also provide a prepotential formulation for $\mathcal{O}(4+n)$ multiplets. Sections 8, 9 and 10 are devoted to superform formulations of the BF, abelian and non-abelian Chern-Simons actions, respectively. In section 11 we describe multiplets with gauged central charge in conformal superspace by giving their superform formulations. In particular, the linear multiplet with central charge, two-form multiplet and large tensor multiplet are discussed. Section 13 is devoted to a description of the dilaton Weyl multiplet and its variants with the use of superforms. In section 14 we present several procedures to generate higher-order off-shell invariants in supergravity, including higher derivative ones. Concluding comments are given in section 15 .

We have included a number of technical appendices. In appendix A we include a summary of our notation and conventions. In appendix B we derive the superconformal algebra from the algebra of conformal Killing supervector fields of $5 \mathrm{D} \mathcal{N}=1$ Minkowski superspace. In appendix $\mathrm{C}$ we give an alternative covariant derivative algebra based on a new vector covariant derivative with a deformed $S$-supersymmetry transformation. Appendix D describes how our component field conventions relate to those of superconformal tensor calculus. In appendix $\mathrm{E}$ we give the $\mathcal{O}(2)$ multiplet prepotential formulation in harmonic superspace. Appendix F discusses the gauge freedom for the $\mathcal{O}(2)$ multiplet. Finally, in appendix $\mathrm{G}$ we derive prepotentials for the $\mathcal{O}(4+n)$ multiplets in harmonic superspace. 


\section{Conformal superspace in five dimensions}

Conformal superspace in four [38, 39] and three [40] dimensions possesses the following key properties: (i) it gauges the entire superconformal algebra; (ii) the curvature and torsion tensors may be expressed in terms of a single primary superfield; and (iii) the algebra obeys the same basic constraints as those of super Yang-Mills theory. In this section we will show how these properties may be used to develop conformal superspace in five dimensions. We will present the superconformal algebra and the geometric setup for conformal superspace based on gauging the entire algebra. We then show how to constrain the geometry to describe superconformal gravity by constraining its covariant derivative algebra to be expressed in terms of a single primary superfield, the super Weyl tensor. We conclude the section by discussing an application and turning on a Yang-Mills multiplet in the conformal superspace setting.

\subsection{The superconformal algebra}

The bosonic generators of the 5D superconformal algebra $\mathrm{F}^{2}(4)[27]$ include the translation $\left(P_{\hat{a}}\right)$, Lorentz $\left(M_{\hat{a} \hat{b}}\right)$, special conformal $\left(K_{\hat{a}}\right)$, dilatation $(\mathbb{D})$ and $\mathrm{SU}(2)$ generators $\left(J_{i j}\right)$, where $\hat{a}, \hat{b}=0,1,2,3,5$ and $i, j=\underline{1}, \underline{2}$. Their algebra is

$$
\begin{aligned}
{\left[M_{\hat{a} \hat{b}}, M_{\hat{c} \hat{d}}\right] } & =2 \eta_{\hat{c}[\hat{a}} M_{\hat{b}] \hat{d}}-2 \eta_{\hat{d}[\hat{a}} M_{\hat{b}] \hat{c}}, & & \\
{\left[M_{\hat{a} \hat{b}}, P_{\hat{c}}\right] } & =2 \eta_{\hat{c}[\hat{a}} P_{\hat{b}]}, & & {\left[\mathbb{D}, P_{\hat{a}}\right]=P_{\hat{a}}, } \\
{\left[M_{\hat{a} \hat{b}}, K_{\hat{c}}\right] } & =2 \eta_{\hat{c}[\hat{a}} K_{\hat{b}]}, & & {\left[\mathbb{D}, K_{\hat{a}}\right]=-K_{\hat{a}}, } \\
{\left[K_{\hat{a}}, P_{\hat{b}}\right] } & =2 \eta_{\hat{a} \hat{b}} \mathbb{D}+2 M_{\hat{a} \hat{b}}, & & \\
{\left[J^{i j}, J^{k l}\right] } & =\varepsilon^{k(i} J^{j) l}+\varepsilon^{l(i} J^{j) k}, & &
\end{aligned}
$$

with all other commutators vanishing. The superconformal algebra is obtained by extending the translation generator to $P_{\hat{A}}=\left(P_{\hat{a}}, Q_{\hat{\alpha}}^{i}\right)$ and the special conformal generator to $K_{\hat{A}}=\left(K_{\hat{a}}, S_{\hat{\alpha} i}\right)$, where $Q_{\hat{\alpha}}^{i}$ and $S_{\hat{\alpha}}^{i}$ are an imaginary and a real pseudo-Majorana spinor, respectively (see appendix A). ${ }^{5}$ The fermionic generator $Q_{\hat{\alpha}}^{i}$ obeys the algebra

$$
\begin{aligned}
& \left\{Q_{\hat{\alpha}}^{i}, Q_{\hat{\beta}}^{j}\right\}=-2 \mathrm{i} \varepsilon^{i j}\left(\Gamma^{\hat{c}}\right)_{\hat{\alpha} \hat{\beta}} P_{\hat{c}}, \quad\left[Q_{\hat{\alpha}}^{i}, P_{\hat{a}}\right]=0, \quad\left[\mathbb{D}, Q_{\hat{\alpha}}^{i}\right]=\frac{1}{2} Q_{\hat{\alpha}}^{i}, \\
& {\left[M_{\hat{\alpha} \hat{\beta}}, Q_{\hat{\gamma}}^{i}\right]=\varepsilon_{\hat{\gamma}(\hat{\alpha}} Q_{\hat{\beta})}^{i}, \quad\left[J^{i j}, Q_{\hat{\alpha}}^{k}\right]=\varepsilon^{k(i} Q_{\hat{\alpha}}^{j)},}
\end{aligned}
$$

while the generator $S_{\hat{\alpha}}^{i}$ obeys the algebra

$$
\begin{aligned}
& \left\{S_{\hat{\alpha}}^{i}, S_{\hat{\beta}}^{j}\right\}=-2 \mathrm{i} \varepsilon^{i j}\left(\Gamma^{\hat{c}}\right)_{\hat{\alpha} \hat{\beta}} K_{\hat{c}}, \quad\left[S_{\hat{\alpha} i}, K_{\hat{a}}\right]=0, \quad\left[\mathbb{D}, S_{\hat{\alpha} i}\right]=-\frac{1}{2} S_{\hat{\alpha} i},
\end{aligned}
$$

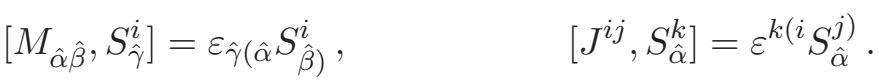

\footnotetext{
${ }^{5}$ Our convention for $S_{\hat{\alpha}}^{i}$ is chosen to match the $4 \mathrm{D}$ convention [39] upon dimensional reduction. This means, for example, that contractions between $K_{\hat{A}}$ and the corresponding gauge parameters, connections, and curvatures must be interpreted with care: for example, $\Lambda^{\hat{A}} K_{\hat{A}}$ should be understood as $\eta^{\hat{\alpha} i} S_{\hat{\alpha} i}+\Lambda_{K}^{\hat{a}} K_{\hat{a}}$ with $\Lambda^{\hat{A}}=\left(\eta^{\hat{\alpha} i}, \Lambda_{K}^{\hat{a}}\right)$, while $\xi^{\hat{A}} P_{\hat{A}}=\xi_{i}^{\hat{\alpha}} Q_{\hat{\alpha}}^{i}+\xi^{\hat{\alpha}} P_{\hat{a}}$ with $\xi^{\hat{A}}=\left(\xi_{i}^{\hat{\alpha}}, \xi^{\hat{a}}\right)$.
} 
Finally, the (anti-)commutators of $K_{\hat{A}}$ with $P_{\hat{A}}$ are

$$
\begin{aligned}
{\left[K_{\hat{a}}, Q_{\hat{\alpha}}^{i}\right] } & =\mathrm{i}\left(\Gamma_{\hat{a}}\right)_{\hat{\alpha}}{ }^{\hat{\beta}} S_{\hat{\beta}}^{i}, \\
\left\{S_{\hat{\alpha} i}, Q_{\hat{\beta}}^{j}\right\} & =2 \varepsilon_{\hat{\alpha} \hat{\beta}} \delta_{i}^{j} \mathbb{D}-4 \delta_{i}^{j} M_{\hat{\alpha} \hat{\beta}}+6 \varepsilon_{\hat{\alpha} \hat{\beta}} J_{i}^{j} .
\end{aligned}
$$

One may explicitly check that the (anti-)commutation relations (2.1) are consistent with the Jacobi identities and thus define a superalgebra. A shorter way to convince oneself of the algebraic structure required is to notice that the (anti-)commutation relations (2.1) follow from the algebra of conformal Killing supervector fields of $5 \mathrm{D} \mathcal{N}=1$ Minkowski superspace [33], see appendix B for the technical details.

\subsection{Gauging the superconformal algebra}

To perform our gauging procedure, we begin with a curved $5 \mathrm{D} \mathcal{N}=1$ superspace $\mathcal{M}^{5 / 8}$ parametrized by local bosonic $(x)$ and fermionic coordinates $\left(\theta_{i}\right)$ :

$$
z^{\hat{M}}=\left(x^{\hat{m}}, \theta_{i}^{\hat{\mu}}\right)
$$

where $\hat{m}=0,1,2,3,5, \hat{\mu}=1, \cdots, 4$ and $i=\underline{1}, \underline{2}$. In order to describe supergravity it is necessary to introduce a vielbein and appropriate connections. However the gauging of the superconformal algebra is made non-trivial due to the fact that the graded commutator of $K_{\hat{A}}$ with $P_{\hat{A}}$ contains generators other than $P_{\hat{A}}$. This requires some of the connections to transform under $K_{\hat{A}}$ into the vielbein. To perform the gauging we will follow closely the approach given in [38-40].

We denote by $X_{\underline{a}}$ the closed subset of generators that do not contain the $P_{\hat{A}}$ generators. The superconformal algebra takes the form of a semidirect product algebra

$$
\begin{aligned}
& {\left[X_{\underline{a}}, X_{\underline{b}}\right\}=-f_{\underline{a} \underline{\underline{c}}}^{\underline{c}} X_{\underline{c}},} \\
& {\left[X_{\underline{a}}, P_{\hat{B}}\right\}=-f_{\underline{a} \hat{B}} \underline{\underline{c}} X_{\underline{c}}-f_{\underline{a} \hat{B}} \hat{C} P_{\hat{C}},} \\
& {\left[P_{\hat{A}}, P_{\hat{B}}\right\}=-f_{\hat{A} \hat{B}} \hat{C} P_{\hat{C}},}
\end{aligned}
$$

where $f_{\hat{A} \hat{B}}^{\hat{C}}$ contains only the constant torsion tensor $f_{\hat{\alpha} \hat{\beta}}^{i j \hat{c}}=\mathscr{T}_{\hat{\alpha}}^{i j \hat{\beta}}=2 \mathrm{i} \varepsilon^{i j}\left(\Gamma^{\hat{c}}\right)_{\hat{\alpha} \hat{\beta}}$. The gauge group associated with the superalgebra generated by $X_{\underline{a}}$ will be denoted $\mathcal{H}$. Now we associate with each generator $X_{\underline{a}}=\left(M_{\hat{a} \hat{b}}, J_{i j}, \mathbb{D}, S_{\hat{\alpha} i}, K_{\hat{a}}\right)$ a connection one-form $\omega^{\underline{a}}=$ $\left(\Omega^{\hat{a} \hat{b}}, \Phi^{i j}, B, \mathfrak{F}^{\hat{\alpha} i}, \mathfrak{F}^{\hat{a}}\right)=\mathrm{d} z^{\hat{M}} \omega_{\hat{M}} \underline{a}$ and with $P_{\hat{A}}$ the vielbein $E^{\hat{A}}=\left(E_{i}^{\hat{\alpha}}, E^{\hat{a}}\right)=\mathrm{d} z^{\hat{M}} E_{\hat{M}}{ }^{\hat{A}}$. Their $\mathcal{H}$-gauge transformations are postulated to be

$$
\begin{aligned}
& \delta_{\mathcal{H}} E^{\hat{A}}=E^{\hat{B}} \Lambda^{\underline{c}} f_{\underline{\underline{c}} \hat{B}}^{\hat{A}}, \\
& \delta_{\mathcal{H}} \omega^{\underline{a}}=\mathrm{d} \Lambda^{\underline{a}}+E^{\hat{B}} \Lambda^{\underline{c}} f_{\underline{\underline{c}} \hat{B}}^{\underline{a}}+\omega^{\underline{b}} \Lambda^{\underline{c}} f_{\underline{c} \underline{\underline{a}}},
\end{aligned}
$$

with $\Lambda^{\underline{a}}$ the gauge parameters.

A superfield $\Phi$ is said to be covariant if it transforms under $\mathcal{H}$ with no derivatives on the parameter $\Lambda^{\underline{a}}$

$$
\delta_{\mathcal{H}} \Phi=\Lambda \Phi:=\Lambda^{\underline{a}} X_{\underline{a}} \Phi .
$$


A superfield $\Phi$ is said to be primary if it is annihilated by the special conformal generators, $K_{\hat{A}} \Phi=0$. From the algebra (2.1), we see that if a superfield is annihilated by $S$-supersymmetry, then it is necessarily primary.

Given a covariant superfield $\Phi$, it is obvious that $\partial_{\hat{M}} \Phi$ is not itself covariant. We are led to introduce the covariant derivative

$$
\nabla=\mathrm{d}-\omega^{\underline{a}} X_{\underline{a}}, \quad \nabla=E^{\hat{A}} \nabla_{\hat{A}}
$$

Its transformation is found to be

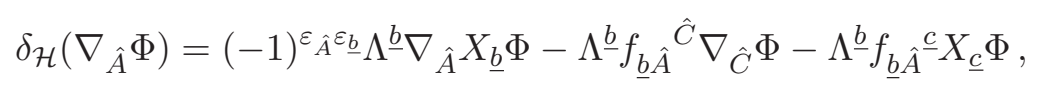

with no derivatives on the gauge parameter $\Lambda^{\underline{a}}$. Rewriting this as $\delta_{\mathcal{H}}\left(\nabla_{\hat{A}} \Phi\right)=\Lambda_{\underline{b}}^{\underline{b}} X_{\underline{b}} \nabla_{\hat{A}} \Phi$, we immediately derive the operator relation

$$
\left[X_{\underline{b}}, \nabla_{\hat{A}}\right\}=-f_{\underline{b} \hat{A}}^{\hat{C}} \nabla_{\hat{C}}-f_{\underline{b} \hat{A}} \hat{c}_{\underline{c}} .
$$

The torsion and curvature tensors appear in the commutator of two covariant derivatives,

$$
\left[\nabla_{\hat{A}}, \nabla_{\hat{B}}\right\}=-\mathscr{T}_{\hat{A} \hat{B}}^{\hat{C}} \nabla_{\hat{C}}-\mathscr{R}_{\hat{A} \hat{B}}^{\underline{c}} X_{\underline{c}}
$$

where the torsion and curvature tensors are defined, respectively, by

$$
\begin{aligned}
\mathscr{T}^{\hat{A}} & :=\frac{1}{2} E^{\hat{C}} \wedge E^{\hat{B}} \mathscr{T}_{\hat{B} \hat{C}} \hat{A}=\mathrm{d} E^{\hat{A}}-E^{\hat{C}} \wedge \omega^{\underline{b}} f_{\underline{b} \hat{C}} \hat{A} \\
\mathscr{R}^{\underline{a}} & :=\frac{1}{2} E^{\hat{C}} \wedge E^{\hat{B} \mathscr{R}_{\hat{B} \hat{C}}} \underline{\underline{a}}=\mathrm{d} \omega^{\underline{a}}-E^{\hat{C}} \wedge \omega^{\underline{b}} f_{\underline{b} \hat{C}} \underline{\underline{a}}-\frac{1}{2} \omega^{\underline{c}} \wedge \omega^{\underline{b}} f_{\underline{\underline{b}}} \underline{\underline{a}} .
\end{aligned}
$$

Using the definition of curvature and torsion (2.10) together with the vielbein and connection transformation rules (2.4), we find

$$
\begin{aligned}
\delta_{\mathcal{H}} \mathscr{T}^{\hat{A}} & =\mathscr{T}^{\hat{C}} \Lambda^{\underline{b}} f_{\underline{b} \hat{C}} \hat{A}-E^{\hat{C}} \wedge E^{\hat{B}} \Lambda^{\underline{a}} f_{\underline{a}} \hat{B}^{-} f_{\underline{f} \hat{C}} \hat{A}, \\
\delta_{\mathcal{H}} \mathscr{R}^{\underline{a}} & =\mathscr{R}^{\underline{c}} \Lambda^{\underline{b}} f_{\underline{b c}}^{\underline{a}}+\mathscr{T}^{\hat{C}} \Lambda^{\underline{b}} f_{\underline{b} \hat{C}^{\underline{a}}}-E^{\hat{D}} \wedge E^{\hat{C}} \Lambda^{\underline{b}} f_{\underline{b} \hat{C}^{-}} f_{\underline{f} \hat{D}^{\underline{a}}},
\end{aligned}
$$

indicating that the torsion and curvature superfields are covariant. Writing the transformation rules as $\delta_{\mathcal{H}} \mathscr{T}^{\hat{A}}=\Lambda^{\underline{a}} X_{\underline{a}} \mathscr{T}^{\hat{A}}, \delta_{\mathcal{H}} \mathscr{R}^{\hat{A}}=\Lambda^{\underline{a}} X_{\underline{a}} \mathscr{R}^{\hat{A}}$ and $\delta_{\mathcal{H}} E^{\hat{A}}=\Lambda^{\underline{b}} X_{\underline{b}} E^{\hat{A}}$ leads to the action of $X_{\underline{a}}$ on the torsion and curvature:

$$
\begin{aligned}
& X_{\underline{a}} \mathscr{T}_{\hat{B} \hat{C}} \hat{D}=-(-1)^{\varepsilon_{a}}\left(\varepsilon_{\hat{B}}+\varepsilon_{\hat{C}}\right) \mathscr{T}_{\hat{B} \hat{C}}{ }^{\hat{E}} f_{\hat{E} \underline{a}} \hat{D}-2 f_{\underline{a}[\hat{B}} \hat{E} \mathscr{T}_{|\hat{E}| \hat{C}\}} \hat{D}-2 f_{\underline{a}[\hat{B}} f_{|\underline{e}| \hat{C}\}} \hat{D}, \\
& X_{\underline{a}} \mathscr{R}_{\hat{B} \hat{C}} \underline{d}=-(-1)^{\varepsilon_{a}\left(\varepsilon_{\hat{B}}+\varepsilon_{\hat{C}}\right)}\left(\mathscr{T}_{\hat{B} \hat{C}}^{\hat{E}} f_{\hat{E} \underline{\underline{a}}}^{\underline{d}}+\mathscr{R}_{\hat{B} \hat{C}} \underline{e}^{\underline{e a}} \underline{f}^{\underline{d}}\right)-2 f_{\underline{a}[\hat{B}} \hat{E} \mathscr{R}_{|\hat{E}| \hat{C}\}} \underline{d} \\
& -2 f_{\underline{a}[\hat{B}} \underline{\underline{e}} f_{|\underline{e}| \hat{C}\}} \underline{d} \text {. }
\end{aligned}
$$

One can show that the above results are the necessary conditions for the Jacobi identity involving two $\nabla$ 's

$$
0=\left[X_{\underline{a}},\left[\nabla_{\hat{B}}, \nabla_{\hat{C}}\right\}\right\}+(\text { graded cyclic permutations })
$$


to be identically satisfied. The Bianchi identities

$$
0=\left[\nabla_{\hat{A}},\left[\nabla_{\hat{B}}, \nabla_{\hat{C}}\right\}\right\}+\text { (graded cyclic permutations) }
$$

can also be shown to be satisfied identically. Therefore, we have a consistent algebraic structure

$$
\begin{aligned}
{\left[X_{\underline{a}}, X_{\underline{b}}\right\} } & =-f_{\underline{a b}} \underline{\underline{c}} X_{\underline{c}}, \\
{\left[X_{\underline{a}}, \nabla_{\hat{B}}\right\} } & =-f_{\underline{a} \hat{B}}^{\hat{C}} \nabla_{\hat{C}}-f_{\underline{a} \hat{B}} \underline{\underline{c}} X_{\underline{c}}, \\
{\left[\nabla_{\hat{A}}, \nabla_{\hat{B}}\right\} } & =-\mathscr{T}_{\hat{A} \hat{B}} \hat{C} \nabla_{\hat{C}}-\mathscr{R}_{\hat{A} \hat{B}} \underline{c} X_{\underline{c}},
\end{aligned}
$$

which satisfies all the Jacobi identities. In the flat space limit the curvature vanishes and the torsion becomes the usual constant torsion, so that the algebra (2.15) exactly matches the superconformal algebra that we started with, in which $P_{\hat{A}}$ is replaced with $\nabla_{\hat{A}}$. The curved case involves a so-called soft algebra, where some of the structure constants have been replaced by structure functions, corresponding to the introduction of torsion and curvature. The superconformal algebra is then said to be "gauged" in this sense.

The full set of operators $\left(\nabla_{\hat{A}}, X_{\underline{a}}\right)$ generates the conformal supergravity gauge group $\mathcal{G}$. The form of the covariant derivative suggests that we should extend the usual diffeomorphisms $\delta_{\text {gct }}$ into covariant diffeomorphisms

$$
\delta_{\text {cgct }}\left(\xi^{\hat{A}}\right):=\delta_{\text {gct }}\left(\xi^{\hat{A}} E_{\hat{A}}^{\hat{M}}\right)-\delta_{\mathcal{H}}\left(\xi^{\hat{A}} \omega_{\hat{A}}^{\underline{a}}\right)
$$

where $\delta_{\text {gct }}\left(\xi^{\hat{M}}\right)$ acts on scalars under diffeomorphisms as

$$
\delta_{\mathrm{gct}} \Phi=\xi^{\hat{M}} \partial_{\hat{M}} \Phi
$$

The full conformal supergravity gauge group $\mathcal{G}$ is then generated by

$$
\mathcal{K}=\xi^{\hat{C}} \nabla_{\hat{C}}+\Lambda^{\underline{a}} X_{\underline{a}} .
$$

If a superfield $\Phi$ is a scalar under diffeomorphisms and covariant under the group $\mathcal{H}$, then its transformation under the full supergravity gauge group $\mathcal{G}$ is

$$
\delta_{\mathcal{G}} \Phi=\mathcal{K} \Phi=\xi^{\hat{C}} \nabla_{\hat{C}} \Phi+\Lambda^{\underline{a}} X_{\underline{a}} \Phi .
$$

It is a straightforward exercise to show that the vielbein and connection one-forms transform as

$$
\begin{aligned}
& \delta_{\mathcal{G}} E^{\hat{A}}=\mathrm{d} \xi^{\hat{A}}+E^{\hat{B}} \Lambda^{\underline{c}} f_{\underline{c} \hat{B}} \hat{A}+\omega^{\underline{b}} \underline{\xi^{\hat{C}}} f_{\hat{C} \underline{b}}{ }^{\hat{A}}+E^{\hat{B}} \xi^{\hat{C}} \mathscr{T}_{\hat{C} \hat{B}} \hat{A}, \\
& \delta_{\mathcal{G}} \omega^{\underline{a}}=\mathrm{d} \Lambda^{\underline{a}}+\omega^{\underline{b}} \Lambda^{\underline{c}} f_{\underline{c} \underline{\underline{b}}}^{\underline{a}}+\omega^{\underline{b}} \xi^{\hat{C}} f_{\hat{C} \underline{b}}^{\underline{a}}+E^{\hat{B}} \Lambda^{\underline{c}} f_{\underline{c} \hat{B}}^{\underline{a}}+E^{\hat{B}} \xi^{\hat{C}} R_{\hat{C} \hat{B}} \underline{a} .
\end{aligned}
$$

From this definition, one can check that the covariant derivative transforms as

$$
\delta_{\mathcal{G}} \nabla_{\hat{A}}=\left[\mathcal{K}, \nabla_{\hat{A}}\right]
$$


provided we interpret

$$
\begin{aligned}
& \nabla_{\hat{A}} \xi^{\hat{B}}:=E_{\hat{A}} \xi^{\hat{B}}+\omega_{\hat{A}}^{\underline{c} \xi^{\hat{D}}} f_{\hat{D} \underline{c}}^{\hat{B}}, \\
& \nabla_{\hat{A}} \Lambda^{\underline{b}}:=E_{\hat{A}} \Lambda^{\underline{b}}+\omega_{\hat{A}^{\underline{c}}} \xi^{\hat{D}} f_{\hat{D}_{\underline{c}}^{\underline{b}}}+\omega_{\hat{A}}{ }^{\underline{c}} \Lambda^{\underline{d}} f_{\underline{d c}}^{\underline{b}} .
\end{aligned}
$$

We can summarize the superspace geometry of conformal supergravity as follows. The covariant derivatives have the form

$$
\nabla_{\hat{A}}=E_{\hat{A}}-\omega_{\hat{A}} X_{\underline{b}}=E_{\hat{A}}-\frac{1}{2} \Omega_{\hat{A}}^{\hat{A} \hat{b}} M_{\hat{a} \hat{b}}-\Phi_{\hat{A}}^{k l} J_{k l}-B_{\hat{A}} \mathbb{D}-\mathfrak{F}_{\hat{A}} \hat{B}_{\hat{B}} .
$$

The action of the generators on the covariant derivatives, eq. (2.15b), resembles that for the $P_{\hat{A}}$ generators given in (2.1). The supergravity gauge group is generated by local transformations of the form (2.21) where

$$
\mathcal{K}=\xi^{\hat{C}} \nabla_{\hat{C}}+\frac{1}{2} \Lambda^{\hat{c} \hat{d}} M_{\hat{c} \hat{d}}+\Lambda^{k l} J_{k l}+\sigma \mathbb{D}+\Lambda^{\hat{A}} K_{\hat{A}}
$$

and the gauge parameters satisfy natural reality conditions. The covariant derivatives satisfy the (anti-)commutation relations

$$
\begin{aligned}
{\left[\nabla_{\hat{A}}, \nabla_{\hat{B}}\right\}=} & -\mathscr{T}_{\hat{A} \hat{B}}{ }^{\hat{C}} \nabla_{\hat{C}}-\frac{1}{2} \mathscr{R}(M)_{\hat{A} \hat{B}}{ }^{\hat{c}} M_{\hat{c} \hat{d}}-\mathscr{R}(J)_{\hat{A} \hat{B}} k l J_{k l} \\
& -\mathscr{R}(\mathbb{D})_{\hat{A} \hat{B}} \mathbb{D}-\mathscr{R}(S)_{\hat{A} \hat{B}}^{\hat{\gamma} k} S_{\hat{\gamma} k}-\mathscr{R}(K)_{\hat{A} \hat{B}}{ }^{\hat{c}} K_{\hat{c}}
\end{aligned}
$$

where the torsion and curvature tensors are given by

$$
\begin{aligned}
\mathscr{T}^{\hat{a}} & =\mathrm{d} E^{\hat{a}}+E^{\hat{b}} \wedge \Omega_{\hat{b}}^{\hat{a}}+E^{\hat{a}} \wedge B \\
\mathscr{T}_{i}^{\hat{\alpha}} & =\mathrm{d} E_{i}^{\hat{\alpha}}+2 E_{i}^{\hat{\beta}} \wedge \Omega_{\hat{\beta}^{\hat{\alpha}}}+\frac{1}{2} E_{i}^{\hat{\alpha}} \wedge B-E^{\hat{\alpha} j} \wedge \Phi_{j i}-\mathrm{i} E^{\hat{c}} \wedge \mathfrak{F}_{i}^{\hat{\beta}}\left(\Gamma_{\hat{c}}\right)_{\hat{\beta}}^{\hat{\alpha}}, \\
\mathscr{R}(\mathbb{D}) & =\mathrm{d} B+2 E^{\hat{a}} \wedge \mathfrak{F}_{\hat{a}}-2 E_{i}^{\hat{\alpha}} \wedge \mathfrak{F}_{\hat{\alpha}}^{i}, \\
\mathscr{R}(M)^{\hat{a} \hat{b}} & =\mathrm{d} \Omega^{\hat{a} \hat{b}}+\Omega^{\hat{a} \hat{c}} \wedge \Omega_{\hat{c}}^{\hat{b}}-4 E^{[\hat{a}} \wedge \mathfrak{F}^{\hat{b}]}-4 E_{j}^{\hat{\alpha}} \wedge \mathfrak{F}^{\hat{\beta} j}\left(\Sigma^{\hat{a} \hat{b}}\right)_{\hat{\alpha} \hat{\beta}}, \\
\mathscr{R}(J)^{i j} & =\mathrm{d} \Phi^{i j}-\Phi^{k(i} \wedge \Phi^{j)}{ }_{k}+6 E^{\hat{\alpha}(i} \wedge \mathfrak{F}_{\hat{\alpha}}^{j)} \\
\mathscr{R}(K)^{\hat{a}} & =\mathrm{d} \mathfrak{F}^{\hat{a}}+\mathfrak{F}^{\hat{b}} \wedge \Omega_{\hat{b}}^{\hat{a}}-\mathfrak{F}^{\hat{a}} \wedge B-\mathrm{i} \mathfrak{F}^{\hat{\alpha} k} \wedge \mathfrak{F}_{\hat{\beta} k}\left(\Gamma^{\hat{a}}\right)_{\hat{\alpha}}{ }^{\hat{\beta}}, \\
\mathscr{R}(S)^{\hat{\alpha} i} & =\mathrm{d} \mathfrak{F}^{\hat{\alpha} i}+2 \mathfrak{F}^{\hat{\beta} i} \wedge \Omega_{\hat{\beta}}^{\hat{\alpha}}-\frac{1}{2} \mathfrak{F}^{\hat{\alpha} i} \wedge B-\mathfrak{F}^{\hat{\alpha} j} \wedge \Phi_{j}{ }^{i}-\mathrm{i} E^{\hat{\beta} i} \wedge \mathfrak{F}^{\hat{c}}\left(\Gamma_{\hat{c}}\right)_{\hat{\beta}}{ }^{\hat{\alpha}} .
\end{aligned}
$$

\subsection{Conformal supergravity}

In the conformal superspace approach to supergravity in four [38, 39] and three [40] dimensions, the entire covariant derivative algebra may be expressed in terms of a single primary superfield: the super Weyl tensor for $D=4$ and the super Cotton tensor for $D=3$. We will seek a similar solution in $D=5$ in terms of a single primary superfield, the super Weyl tensor $W_{\hat{\alpha} \hat{\beta}}=W_{\hat{\beta} \hat{\alpha}}[26]$.

In the three- and four-dimensional cases the second ingredient to describe conformal supergravity was to realize that the right constraints for the covariant derivative were such that their algebra obeyed the same constraints as super Yang-Mills theory. Guided by 
the structure of $5 \mathrm{D} \mathcal{N}=1$ super Yang-Mills theory [2, 7, 51], we impose the constraint $\left\{\nabla_{\hat{\alpha}}^{(i}, \nabla_{\hat{\beta}}^{j)}\right\}=0$, which is equivalent to the spinor derivative anti-commutation relation

$$
\left\{\nabla_{\hat{\alpha}}^{i}, \nabla_{\hat{\beta}}^{j}\right\}=-2 \mathrm{i} \varepsilon^{i j}\left(\Gamma^{\hat{c}}\right)_{\hat{\alpha} \hat{\beta}} \nabla_{\hat{c}}-2 \mathrm{i} \varepsilon^{i j} \varepsilon_{\hat{\alpha} \hat{\beta}} \mathscr{W}
$$

where $\mathscr{W}$ is some operator taking values in the superconformal algebra. The Bianchi identities give the other commutators

$$
\begin{aligned}
& {\left[\nabla_{\hat{a}}, \nabla_{\hat{\beta}}^{j}\right]=\left(\Gamma_{\hat{a}}\right)_{\hat{\beta}}^{\hat{\gamma}}\left[\nabla_{\hat{\gamma}}^{j}, \mathscr{W}\right],} \\
& {\left[\nabla_{\hat{a}}, \nabla_{\hat{b}}\right]=-\mathscr{F}_{\hat{a} \hat{b}}=\frac{\mathrm{i}}{4}\left(\Sigma_{\hat{a} \hat{b}}\right)^{\hat{\alpha} \hat{\beta}}\left\{\nabla_{\hat{\alpha}}^{k},\left[\nabla_{\hat{\beta} k}, \mathscr{W}\right]\right\}}
\end{aligned}
$$

and the additional constraint

$$
\left\{\nabla_{\hat{\alpha}}^{(i},\left[\nabla_{\hat{\beta}}^{j)}, \mathscr{W}\right]\right\}=\frac{1}{4} \varepsilon_{\hat{\alpha} \hat{\beta}}\left\{\nabla^{\gamma(i},\left[\nabla_{\gamma}^{j)}, \mathscr{W}\right]\right\}
$$

In analogy to conformal superspace in four dimensions [38, 39], we constrain the form of the operator $\mathscr{W}$ to be

$$
\mathscr{W}=W^{\hat{\alpha} \hat{\beta}} M_{\hat{\alpha} \hat{\beta}}+W(S)^{\hat{\alpha} i} S_{\hat{\alpha} i}+W(K)^{\hat{b}} K_{\hat{b}},
$$

where $W_{\hat{\alpha} \hat{\beta}}$ is a symmetric dimension- 1 primary superfield. One can show that the Bianchi identity (2.28) is identically satisfied for

$$
\mathscr{W}=W^{\hat{\alpha} \hat{\beta}} M_{\hat{\alpha} \hat{\beta}}-\frac{1}{10}\left(\nabla_{\hat{\beta}}^{i} W^{\hat{\alpha} \hat{\beta}}\right) S_{\hat{\alpha} i}-\frac{1}{4}\left(\nabla^{\hat{a}} W_{\hat{a} \hat{b}}\right) K^{\hat{b}},
$$

provided $W_{\hat{\alpha} \hat{\beta}}$ satisfies

$$
\nabla_{\hat{\gamma}}^{k} W_{\hat{\alpha} \hat{\beta}}=\nabla_{(\hat{\alpha}}^{k} W_{\hat{\beta} \hat{\gamma})}+\frac{2}{5} \varepsilon_{\hat{\gamma}(\hat{\alpha}} \nabla^{\hat{\delta} k} W_{\hat{\beta}) \hat{\delta}} .
$$

It is convenient to introduce higher dimension descendant superfields constructed from spinor derivatives of $W_{\hat{\alpha} \hat{\beta}}$. At dimension-3/2, we introduce

$$
W_{\hat{\alpha} \hat{\beta} \hat{\gamma}}^{k}:=\nabla_{(\hat{\alpha}}^{k} W_{\hat{\beta} \hat{\gamma})}, \quad X_{\hat{\alpha}}^{i}:=\frac{2}{5} \nabla^{\hat{\beta} i} W_{\hat{\beta} \hat{\alpha}},
$$

and at dimension-2, we choose

$$
\begin{aligned}
W_{\hat{\alpha} \hat{\beta} \hat{\gamma} \hat{\delta}} & :=\nabla_{(\hat{\alpha}}^{k} W_{\hat{\beta} \hat{\gamma} \hat{\delta}) k}, \\
Y & :=\mathrm{i} \nabla^{\hat{\gamma} k} X_{\hat{\gamma} k} .
\end{aligned}
$$

One can check that only these superfields and their vector derivatives appear upon taking successive spinor derivatives of $W_{\hat{\alpha} \hat{\beta}}$. Specific relations we will need later are given below:

$$
\begin{aligned}
\nabla_{\hat{\gamma}}^{k} W_{\hat{\alpha} \hat{\beta}} & =W_{\hat{\alpha} \hat{\beta} \hat{\gamma}}^{k}+\varepsilon_{\hat{\gamma}(\hat{\alpha}} X_{\hat{\beta})}^{k}, \\
\nabla_{\hat{\alpha}}^{i} X_{\hat{\beta}}^{j} & =X_{\hat{\alpha} \hat{\beta}}^{i j}+\frac{\mathrm{i}}{8} \varepsilon^{i j}\left(\varepsilon_{\hat{\alpha} \hat{\beta}} Y+4 \varepsilon^{\hat{a} \hat{b} \hat{c} \hat{d} \hat{e}}\left(\Sigma_{\hat{a} \hat{b}}\right)_{\hat{\alpha} \hat{\beta}} \nabla_{\hat{c}} W_{\hat{d} \hat{e}}-4\left(\Gamma^{\hat{b}}\right)_{\hat{\alpha} \hat{\beta}} \nabla^{\hat{a}} W_{\hat{a} \hat{b}}\right)
\end{aligned}
$$




$$
\begin{aligned}
& \nabla_{\hat{\alpha}}^{i} W_{\hat{\beta} \hat{\gamma} \hat{\delta}}^{j}=-\frac{1}{2} \varepsilon^{i j}\left(W_{\hat{\alpha} \hat{\beta} \hat{\gamma} \hat{\delta}}+3 \mathrm{i} \nabla_{\hat{\alpha}(\hat{\beta}} W_{\hat{\gamma} \hat{\delta})}-\frac{3 \mathrm{i}}{4} \varepsilon^{\hat{a} \hat{b} \hat{c} \hat{d} \hat{e}} \varepsilon_{\hat{\alpha}(\hat{\beta}}\left(\Sigma_{\hat{a} \hat{b}}\right)_{\hat{\gamma} \hat{\delta})} \nabla_{\hat{c}} W_{\hat{d} \hat{e}}\right) \\
& -\frac{3}{2} \varepsilon_{\hat{\alpha}(\hat{\beta}} X_{\hat{\gamma} \hat{\delta})}{ }^{i j} \\
& \nabla_{\hat{\alpha}}^{i} W_{\hat{\beta} \hat{\gamma} \hat{\delta} \hat{\rho}}=-4 \mathrm{i} \nabla_{\hat{\alpha}(\hat{\beta}} W_{\hat{\gamma} \hat{\delta} \hat{\rho})}^{i}-12 \mathrm{i} \varepsilon_{\hat{\alpha}(\hat{\beta}}\left(\nabla_{\hat{\gamma}}^{\hat{\tau}} W_{\hat{\delta} \hat{\rho}) \hat{\tau}}{ }^{i}+W_{\hat{\gamma} \hat{\delta}} X_{\hat{\rho})}^{i}-2 W_{\hat{\gamma}}^{\hat{\tau}} W_{\hat{\delta} \hat{\rho}) \hat{\tau}}{ }^{i}\right), \\
& \nabla_{\hat{\alpha}}^{i} X_{\hat{\beta} \hat{\gamma}}{ }^{j k}=\varepsilon^{i(j}\left(2 \mathrm{i} \nabla_{\hat{\alpha}}^{\hat{\delta}} W_{\hat{\beta} \hat{\gamma} \hat{\delta}}^{k)}+2 \mathrm{i} \nabla_{(\hat{\beta}}^{\hat{\delta}} W_{\hat{\gamma}) \hat{\alpha} \hat{\delta}}^{k)}-\mathrm{i} \nabla_{\hat{\alpha}(\hat{\beta}} X_{\hat{\gamma})}^{k)}-\mathrm{i} \varepsilon_{\hat{\alpha}(\hat{\beta}} \nabla_{\hat{\gamma})}{ }^{\hat{\delta}} X_{\hat{\delta}}^{k)}\right. \\
& \left.+6 \mathrm{i} W_{(\hat{\alpha} \hat{\beta}} X_{\hat{\gamma})}^{k)}-12 \mathrm{i} W_{(\hat{\alpha}}^{\hat{\delta}} W_{\hat{\beta} \hat{\gamma}) \hat{\delta}}^{k)}\right), \\
& \nabla_{\hat{\alpha}}^{i} Y=8 \nabla_{\hat{\alpha}}^{\hat{\gamma}} X_{\hat{\gamma}}^{i} \text {. }
\end{aligned}
$$

These descendant superfields transform under $S$-supersymmetry as

$$
\begin{array}{rlrl}
S_{\hat{\alpha} i} W_{\hat{\beta} \hat{\gamma} \hat{\delta}}^{j} & =6 \delta_{i}^{j} \varepsilon_{\hat{\alpha}(\hat{\beta}} W_{\hat{\gamma} \hat{\delta})}, & S_{\hat{\alpha} i} X_{\hat{\beta}}^{j}=4 \delta_{i}^{j} W_{\hat{\alpha} \hat{\beta}}, \\
S_{\hat{\alpha} i} W_{\hat{\beta} \hat{\gamma} \hat{\delta} \hat{\rho}}=24 \varepsilon_{\hat{\alpha}(\hat{\beta}} W_{\hat{\gamma} \hat{\delta} \hat{\rho})^{i}}, & S_{\hat{\alpha} i} Y=8 \mathrm{i} X_{\hat{\alpha} i}, \\
S_{\hat{\alpha} i} X_{\hat{\beta} \hat{\gamma}}{ }^{j k}=-4 \delta_{i}^{(j} W_{\hat{\alpha} \hat{\beta} \hat{\gamma}}^{k)}+4 \delta_{i}^{(j} \varepsilon_{\hat{\alpha}(\hat{\beta}} X_{\hat{\gamma})}^{k)} . &
\end{array}
$$

In terms of these superfields, we can now construct the algebra of covariant derivatives for $5 \mathrm{D}$ conformal supergravity:

$$
\begin{aligned}
& \left\{\nabla_{\hat{\alpha}}^{i}, \nabla_{\hat{\beta}}^{j}\right\}=-2 \mathrm{i} \varepsilon^{i j}\left(\Gamma^{\hat{c}}\right)_{\hat{\alpha} \hat{\beta}} \nabla_{\hat{c}}-2 \mathrm{i} \varepsilon^{i j} \varepsilon_{\hat{\alpha} \hat{\beta}} W^{\hat{\gamma} \hat{\delta}} M_{\hat{\gamma} \hat{\delta}}-\frac{\mathrm{i}}{2} \varepsilon^{i j} \varepsilon_{\hat{\alpha} \hat{\beta}} X^{\hat{\gamma} k} S_{\hat{\gamma} k} \\
& +\frac{\mathrm{i}}{2} \varepsilon^{i j} \varepsilon_{\hat{\alpha} \hat{\beta}}\left(\nabla^{\hat{a}} W_{\hat{a} \hat{b}}\right) K^{\hat{b}}, \\
& {\left[\nabla_{\hat{a}}, \nabla_{\hat{\beta}}^{j}\right]=\left(\Gamma_{\hat{a}}\right)_{\hat{\beta}}^{\hat{\gamma}}\left(W_{\hat{\gamma} \hat{\delta}} \nabla^{\hat{\delta} j}+\frac{1}{2} X_{\hat{\gamma}}^{j} \mathbb{D}+W_{\hat{\gamma} \hat{\delta} \hat{\rho}}^{j} M^{\hat{\delta} \hat{\rho}}+\frac{3}{2} X_{\hat{\gamma}}^{k} J_{k}^{j}\right.} \\
& \left.-\frac{1}{4}\left(\nabla_{\hat{\gamma}}^{j} X_{k}^{\hat{\delta}}\right) S_{\hat{\delta}}^{k}+\frac{\mathrm{i}}{4}\left(\Gamma^{\hat{c}}\right)_{\hat{\gamma}}^{\hat{\delta}}\left(\nabla^{\hat{b}} W_{\hat{b} \hat{c}}\right) S_{\hat{\delta}}^{j}-\frac{1}{4}\left(\nabla_{\hat{\gamma}}^{j} \nabla^{\hat{c}} W_{\hat{c} \hat{b}}\right) K^{\hat{b}}\right) \text {, } \\
& {\left[\nabla_{\hat{a}}, \nabla_{\hat{b}}\right]=-\mathscr{T}_{\hat{a} \hat{b}}^{\hat{c}} \nabla_{\hat{c}}-\mathscr{T}_{\hat{a} \hat{b} k}^{\hat{\gamma}} \nabla_{\hat{\gamma}}^{k}-\frac{1}{2} \mathscr{R}(M)_{\hat{a} \hat{b}}^{\hat{c} \hat{d}} M_{\hat{c} \hat{d}}-\mathscr{R}(J)_{\hat{a} \hat{b}}^{i j} J_{i j}-\mathscr{R}(\mathbb{D})_{\hat{a} \hat{b}} \mathbb{D}} \\
& -\mathscr{R}(S)_{\hat{a} \hat{b}}{ }^{\hat{\gamma} k} S_{\hat{\gamma} k}-\mathscr{R}(K)_{\hat{a} \hat{b}}{ }^{\hat{c}} K_{\hat{c}}
\end{aligned}
$$

where

$$
\begin{aligned}
& \mathscr{T}_{\hat{a} \hat{b}}{ }^{\hat{c}}=-\frac{1}{2} \varepsilon_{\hat{a} \hat{b}} \hat{c} \hat{d} \hat{e} W_{\hat{d} \hat{e}}, \\
& \mathscr{T}_{\hat{a} \hat{b} k}^{\hat{\gamma}}=-\frac{\mathrm{i}}{2} \nabla_{k}^{\hat{\gamma}} W_{\hat{a} \hat{b}}=-\frac{\mathrm{i}}{2}\left(\Sigma_{\hat{a} \hat{b}}\right)_{\hat{\alpha} \hat{\beta}} W^{\hat{\alpha} \hat{\beta} \hat{\gamma}} k-\frac{\mathrm{i}}{2}\left(\Sigma_{\hat{a} \hat{b}}\right)^{\hat{\beta} \hat{\gamma}} X_{\hat{\beta} k}, \\
& \mathscr{R}(M)_{\hat{a} \hat{b}} \hat{\hat{b}} \hat{d}=-\frac{\mathrm{i}}{4}\left(\Sigma_{\hat{a} \hat{b}}\right)^{\hat{\alpha} \hat{\beta}}\left(\Sigma^{\hat{c} \hat{d}}\right)^{\hat{\gamma} \hat{\delta}} \nabla_{\hat{\alpha}}^{k} \nabla_{\hat{\beta} k} W_{\hat{\gamma} \hat{\delta}}+\frac{\mathrm{i}}{10} \nabla^{\hat{\gamma} k} \nabla_{k}^{\hat{\delta}} W_{\hat{\gamma} \hat{\delta}} \delta_{[\hat{a}}^{\hat{c}} \delta_{\hat{b}]}^{\hat{d}}
\end{aligned}
$$

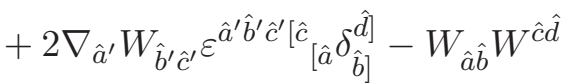

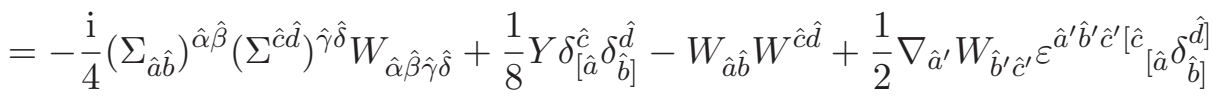

$$
\begin{aligned}
& +\frac{1}{4} \varepsilon_{\hat{a} \hat{b}} \hat{c} \hat{e} \hat{e} \nabla^{\hat{f}} W_{\hat{f} \hat{e}}+\frac{1}{4} \varepsilon^{\hat{c} \hat{d} \hat{e} \hat{f}}{ }_{[\hat{a}} \nabla_{\hat{b}]} W_{\hat{e} \hat{f}}-\frac{1}{2} \nabla^{\hat{e}} W^{\hat{f}[\hat{c}} \varepsilon^{\hat{d}]}{ }_{\hat{a} \hat{b} \hat{e} \hat{f}}, \\
& \mathscr{R}(J)_{\hat{a} \hat{b}}^{k l}=-\frac{3 \mathrm{i}}{4}\left(\Sigma_{\hat{a} \hat{b}}\right)^{\hat{\alpha} \hat{\beta}} X_{\hat{\alpha} \hat{\beta}}^{k l}=\frac{3 \mathrm{i}}{16}\left(\Sigma_{\hat{a} \hat{b}}\right)^{\hat{\alpha} \hat{\beta}} \nabla^{\hat{\gamma}(k} \nabla_{\hat{\gamma}}^{l)} W_{\hat{\alpha} \hat{\beta}},
\end{aligned}
$$




$$
\begin{aligned}
\mathscr{R}(\mathbb{D})_{\hat{a} \hat{b}}= & -\frac{\mathrm{i}}{4}\left(\Sigma_{\hat{a} \hat{b}}\right)^{\hat{\alpha} \hat{\beta}} \nabla_{\hat{\alpha}}^{k} X_{\hat{\beta} k}=-\frac{1}{2} \varepsilon_{\hat{a} \hat{b} \hat{c} \hat{d} \hat{e}} \nabla^{\hat{c}} W^{\hat{d} \hat{e}}, \\
\mathscr{R}(S)_{\hat{a} \hat{b}} \hat{\gamma} k= & -\frac{\mathrm{i}}{16}\left(\Sigma_{\hat{a} \hat{b}}\right)^{\hat{\alpha} \hat{\beta}}\left(\nabla_{\hat{\alpha}}^{j} \nabla_{\hat{\beta} j} X^{\hat{\gamma} k}+2 \mathrm{i}\left(\Gamma^{\hat{c}}\right)_{\hat{\alpha}}^{\hat{\gamma}} \nabla^{\hat{d}} \nabla_{\hat{\beta}}^{k} W_{\hat{d} \hat{c}}-4 \mathrm{i} W_{\hat{\alpha} \hat{\beta}} X^{\hat{\gamma} k}\right) \\
= & -\frac{1}{16} \varepsilon^{\hat{\gamma} \hat{\rho}}\left(\Sigma_{\hat{a} \hat{b}}\right)^{\hat{\alpha} \hat{\beta}}\left(2 \nabla_{\hat{\alpha}}^{\hat{\delta}} W_{\hat{\beta} \hat{\delta} \hat{\rho}}^{k}+6 \nabla_{\hat{\rho}}^{\hat{\delta}} W_{\hat{\alpha} \hat{\beta} \hat{\delta}}^{k}+\varepsilon_{\hat{\rho} \hat{\alpha}} \nabla_{\hat{\beta}}^{\hat{\delta}} X_{\hat{\delta}}^{k}+3 \nabla_{\hat{\rho} \hat{\alpha}} X_{\hat{\beta}}^{k}\right. \\
& \left.-12 W_{(\hat{\rho}}^{\hat{\delta}} W_{\hat{\alpha} \hat{\beta}) \hat{\delta}}^{k}+6 W_{(\hat{\alpha} \hat{\beta}} X_{\hat{\rho})}^{k}\right)-\frac{1}{4} W_{\hat{a} \hat{b}} X^{\hat{\gamma} k} \\
\mathscr{R}(K)_{\hat{a} \hat{b}} \hat{c}= & \frac{\mathrm{i}}{16}\left(\Sigma_{\hat{a} \hat{b}}\right)^{\hat{\alpha} \hat{\beta}}\left(\nabla_{(\hat{\alpha}}^{k} \nabla_{\hat{\beta}) k} \nabla_{\hat{d}} W^{\hat{d} \hat{c}}-4 \mathrm{i} W_{\hat{\alpha} \hat{\beta}} \nabla_{\hat{d}} W^{\hat{d} \hat{c}}\right) \\
= & \frac{\mathrm{i}}{16}\left(\sum_{\hat{a} \hat{b}}\right)^{\hat{\alpha} \hat{\beta}}\left(\nabla_{\hat{d}} \nabla_{(\hat{\alpha}}^{k} \nabla_{\hat{\beta}) k} W^{\hat{d} \hat{c}}+\frac{\mathrm{i}}{8} \varepsilon^{\hat{c} \hat{e} \hat{f} \hat{g} \hat{h}}\left(\Sigma_{\hat{\hat{e}} \hat{f}}\right)_{\hat{\alpha} \hat{\beta}} Y W_{\hat{g} \hat{h}}\right. \\
& -\left(\Gamma^{\hat{c}}\right)_{\hat{\delta}}^{\hat{\rho}} W^{\hat{\gamma} \hat{\delta}} \nabla_{\hat{\gamma}}^{k} \nabla_{\hat{\alpha} k} W_{\hat{\beta} \hat{\rho}}+\left(\Gamma^{\hat{c}}\right)_{\hat{\alpha}}^{\hat{\rho}} W^{\hat{\gamma} \hat{\delta}} \nabla_{\hat{\gamma}}^{k} \nabla_{\hat{\beta} k} W_{\hat{\delta} \hat{\rho}} \\
& +2 \mathrm{i} W_{\hat{\alpha} \hat{\beta}} \nabla_{\hat{e}} W^{\hat{e} \hat{c}}-\mathrm{i}\left(\Sigma^{\hat{e} \hat{f}}\right)_{\hat{\alpha} \hat{\beta}} W_{\hat{f}}^{\hat{c}} \nabla^{\hat{g}} W_{\hat{g} \hat{e}}+3 \mathrm{i}\left(\Sigma^{\hat{c} \hat{e}}\right)_{\hat{\alpha} \hat{\beta}} W_{\hat{e}}^{\hat{g}} \nabla^{\hat{f}} W_{\hat{f} \hat{g}} \\
& -6 \mathrm{i}\left(\Sigma^{\hat{e} \hat{f}}\right)_{\hat{\alpha} \hat{\beta}} W^{\hat{g} \hat{c}} \nabla_{[\hat{e}} W_{\hat{f} \hat{g}]}-3\left(\Gamma^{\hat{c}}\right)^{\hat{\gamma} \hat{\delta}} X_{\hat{\gamma}}^{k} W_{\hat{\alpha} \hat{\beta} \hat{\delta} k}-3\left(\Gamma^{\hat{c}}\right)_{\hat{\alpha}}^{\hat{\delta}} X^{\hat{\gamma} k} W_{\hat{\beta} \hat{\gamma} \hat{\delta} k} \\
& \left.-2\left(\Gamma^{\hat{c}}\right)^{\hat{\delta} \hat{\rho}} W_{\hat{\delta}(\hat{\alpha}}^{\hat{\gamma} k} W_{\hat{\beta}) \hat{\rho} \hat{\gamma} k}-2\left(\Gamma^{\hat{c}}\right)_{\hat{\alpha}}^{\hat{\rho}} W_{\hat{\beta}}^{\hat{\gamma} \hat{\gamma} k} W_{\hat{\gamma} \hat{\delta} \hat{\rho} k}-4 \mathrm{i} W_{\hat{\alpha} \hat{\beta}} \nabla_{\hat{d}} W^{\hat{d} \hat{c}}\right) .
\end{aligned}
$$

Despite possessing a larger structure group, the covariant derivative algebra is more compact than that of $\mathrm{SU}(2)$ superspace [26]. This provides a significant advantage in performing superspace calculations.

\subsection{Full superspace actions}

Given the geometry we have described, it is immediately apparent that one may construct an action principle involving a full superspace integral

$$
S[\mathcal{L}]=\int \mathrm{d}^{5 \mid 8} z E \mathcal{L}, \quad \mathrm{d}^{5 \mid 8} z:=\mathrm{d}^{5} x \mathrm{~d}^{8} \theta, \quad E:=\operatorname{Ber}\left(E_{\hat{M}}^{\hat{A}}\right)
$$

where $\mathcal{L}$ is a primary superspace Lagrangian of dimension +1 .

For later applications, it will be important to know the rule for integrating by parts in full superspace. It is given by

$$
\begin{aligned}
\int \mathrm{d}^{5 \mid 8} z E(-1)^{\varepsilon_{\hat{A}}} \nabla_{\hat{A}} V^{\hat{A}}=\int \mathrm{d}^{5 \mid 8} z E\{ & -(-1)^{\varepsilon_{\hat{A}}}\left(\mathfrak{F}_{\hat{A}}^{\hat{b}} K_{\hat{b}} V^{\hat{A}}+\mathfrak{F}_{\hat{A}}^{\hat{\beta} k} S_{\hat{\beta} k} V^{\hat{A}}\right) \\
& \left.+\mathrm{i} \mathfrak{F}_{\hat{\gamma} k}{ }^{\hat{\beta} k} V^{\hat{a}}\left(\Gamma_{\hat{a}}\right)_{\hat{\beta}}^{\hat{\gamma}}\right\}
\end{aligned}
$$

where $V^{\hat{A}}$ transforms as a Lorentz and $\mathrm{SU}(2)$ tensor with $\mathbb{D} V^{\hat{a}}=0$ and $\mathbb{D} V_{i}^{\hat{\alpha}}=\frac{1}{2} V_{i}^{\hat{\alpha}}$.

In the special case where $V^{\hat{A}}$ corresponds to an $S$-invariant vector field $V=V^{\hat{A}} E_{\hat{A}}=$ $V^{\hat{A}} E_{\hat{A}}^{M} \partial_{\hat{M}}$, which requires

$$
S_{\hat{\beta}}^{i} V_{j}^{\hat{\alpha}}=-\mathrm{i} \delta_{j}^{i} V^{\hat{a}}\left(\Gamma_{\hat{a}}\right)_{\hat{\beta}}^{\hat{\alpha}}, \quad S_{\hat{\beta}}^{j} V^{\hat{a}}=0,
$$

we have the simple integration rule

$$
\int \mathrm{d}^{5 \mid 8} z E(-1)^{\varepsilon_{\hat{A}}} \nabla_{\hat{A}} V^{\hat{A}}=0 .
$$




\subsection{Gravitational composite $\mathcal{O}(2)$ multiplet}

As an application of the formalism introduced, we will construct a composite superfield that may be used to generate a supersymmetric completion of an $R^{2}$ term. This composite superfield is constructed in terms of the super Weyl tensor as follows:

$$
H_{\text {Weyl }}^{i j}:=-\frac{\mathrm{i}}{2} W^{\hat{\alpha} \hat{\beta} \hat{\gamma} i} W_{\hat{\alpha} \hat{\beta} \hat{\gamma}}^{j}+\frac{3 \mathrm{i}}{2} W^{\hat{\alpha} \hat{\beta}} X_{\hat{\alpha} \hat{\beta}}^{i j}-\frac{3 \mathrm{i}}{4} X^{\hat{\alpha} i} X_{\hat{\alpha}}^{j}=H_{\text {Weyl }}^{j i},
$$

where we have used the definitions (2.32). This superfield is real in the sense that $\overline{H_{\text {Weyl }}^{i j}}=$ $\varepsilon_{i k} \varepsilon_{j l} H_{\mathrm{Weyl}}^{k l}$. One can check that $H_{\mathrm{Weyl}}^{i j}$ is primary and obeys the constraint

$$
\nabla_{\hat{\alpha}}^{(i} H_{\mathrm{Weyl}}^{j k)}=0 \text {. }
$$

It corresponds exactly to the composite multiplet $L^{i j}\left[\mathbf{W}^{2}\right]$ constructed by Hanaki, Ohashi, and Tachikawa [29].

This is an example of a covariant real $\mathcal{O}(2)$ multiplet, which will be introduced in section 5. The structure of (2.41) is completely analogous to that of the composite $\mathcal{O}(2)$ multiplet associated with the Yang-Mills multiplet given in [2], see the next subsection. The supersymmetric $R^{2}$-invariant of [29] may be constructed straightforwardly in superspace using (2.41) and the BF action.

\subsection{Turning on the Yang-Mills multiplet}

Let us conclude this subsection by presenting a Yang-Mills multiplet in conformal superspace. To describe such a non-abelian vector multiplet, the covariant derivative $\nabla=E^{\hat{A}} \nabla_{\hat{A}}$ has to be replaced with a gauge covariant one,

$$
\boldsymbol{\nabla}=E^{\hat{A}} \nabla_{\hat{A}}, \quad \nabla_{\hat{A}}:=\nabla_{\hat{A}}-\mathrm{i} \boldsymbol{V}_{\hat{A}} .
$$

Here the gauge connection one-form $\boldsymbol{V}=E^{\hat{A}} \boldsymbol{V}_{\hat{A}}$ takes its values in the Lie algebra of the Yang-Mills gauge group, $G_{\mathrm{YM}}$, with its (Hermitian) generators commuting with all the generators of the superconformal algebra. The gauge covariant derivative algebra is

$$
\begin{aligned}
{\left[\nabla_{\hat{A}}, \nabla_{\hat{B}}\right\}=} & -\mathscr{T}_{\hat{A} \hat{B}}{ }^{\hat{C}} \nabla_{\hat{C}}-\frac{1}{2} \mathscr{R}(M)_{\hat{A} \hat{B}}{ }^{\hat{c} \hat{d}} M_{\hat{c} \hat{d}}-\mathscr{R}(J)_{\hat{A} \hat{B}}{ }^{k l} J_{k l}-\mathscr{R}(\mathbb{D})_{\hat{A} \hat{B}} \mathbb{D} \\
& -\mathscr{R}(S)_{\hat{A} \hat{B}}^{\hat{\gamma} k} S_{\hat{\gamma} k}-\mathscr{R}(K)_{\hat{A} \hat{B}}{ }^{\hat{c}} K_{\hat{c}}-\mathrm{i} \boldsymbol{F}_{\hat{A} \hat{B}},
\end{aligned}
$$

where the torsion and curvatures are those of conformal superspace but with $\boldsymbol{F}_{\hat{A} \hat{B}}$ corresponding to the gauge covariant field strength two-form $\boldsymbol{F}=\frac{1}{2} E^{\hat{B}} \wedge E^{\hat{A}} \boldsymbol{F}_{\hat{A} \hat{B}}$. The field strength $\boldsymbol{F}_{\hat{A} \hat{B}}$ satisfies the Bianchi identity

$$
\boldsymbol{\nabla} \boldsymbol{F}=0 \Longleftrightarrow \boldsymbol{\nabla}_{[\hat{A}} \boldsymbol{F}_{\hat{B} \hat{C}\}}+\mathscr{T}_{[\hat{A} \hat{B}}^{\hat{D}} \boldsymbol{F}_{|\hat{D}| \hat{C}\}}=0 .
$$

The Yang-Mills gauge transformation acts on the gauge covariant derivatives $\nabla_{\hat{A}}$ and a matter superfield $U$ (transforming in some representation of the gauge group) as

$$
\boldsymbol{\nabla}_{\hat{A}} \rightarrow \mathrm{e}^{\mathrm{i} \boldsymbol{\tau}} \boldsymbol{\nabla}_{\hat{A}} \mathrm{e}^{-\mathrm{i} \boldsymbol{\tau}}, \quad U \rightarrow U^{\prime}=\mathrm{e}^{\mathrm{i} \boldsymbol{\tau}} U, \quad \boldsymbol{\tau}^{\dagger}=\boldsymbol{\tau},
$$


where the Hermitian gauge parameter $\boldsymbol{\tau}(z)$ takes its values in the Lie algebra of $G_{\mathrm{YM}}$. This implies that the gauge one-form and the field strength transform as follows:

$$
\boldsymbol{V} \rightarrow \mathrm{e}^{\mathrm{i} \boldsymbol{\tau}} \boldsymbol{V} \mathrm{e}^{-\mathrm{i} \boldsymbol{\tau}}+\mathrm{i} \mathrm{e}^{\mathrm{i} \boldsymbol{\tau}} \mathrm{de}^{-\mathrm{i} \boldsymbol{\tau}}, \quad \boldsymbol{F} \rightarrow \mathrm{e}^{\mathrm{i} \boldsymbol{\tau}} \boldsymbol{F} \mathrm{e}^{-\mathrm{i} \boldsymbol{\tau}} .
$$

As in the flat case [7] (see also $[2,51]$ ), some components of the field strength have to be constrained in order to describe an irreducible multiplet. In conformal superspace the right constraint is

$$
\boldsymbol{F}_{\hat{\alpha} \hat{\beta}}^{i j}=2 \mathrm{i} \varepsilon^{i j} \varepsilon_{\hat{\alpha} \hat{\beta}} \boldsymbol{W},
$$

which fixes the remaining components of the field strengths to be

$$
\begin{aligned}
\boldsymbol{F}_{\hat{a} \hat{\beta}}^{j} & =-\left(\Gamma_{\hat{a}}\right)_{\hat{\beta}}^{\hat{\gamma}} \nabla_{\hat{\gamma}}^{j} \boldsymbol{W}, \\
\boldsymbol{F}_{\hat{a} \hat{b}} & =-\frac{\mathrm{i}}{4}\left(\Sigma_{\hat{a} \hat{b}}\right)^{\hat{\alpha} \hat{\beta}}\left(\nabla_{(\hat{\alpha}}^{k} \nabla_{\hat{\beta}) k}-4 \mathrm{i} W_{\hat{\alpha} \hat{\beta}}\right) \boldsymbol{W},
\end{aligned}
$$

where the superfield $\boldsymbol{W}$ is Hermitian, $\boldsymbol{W}^{\dagger}=\boldsymbol{W}$, and obeys the Bianchi identity

$$
\boldsymbol{\nabla}_{\hat{\alpha}}^{(i} \nabla_{\hat{\beta}}^{j)} \boldsymbol{W}=\frac{1}{4} \varepsilon_{\hat{\alpha} \hat{\beta}} \nabla^{\hat{\gamma}(i} \nabla_{\hat{\gamma}}^{j)} \boldsymbol{W}
$$

Moreover, $\boldsymbol{W}$ is a conformal primary of dimension $1, S_{\hat{\alpha}}^{i} \boldsymbol{W}=0$ and $\mathbb{D} \boldsymbol{W}=\boldsymbol{W}$.

Now let $T_{I}$ be the Hermitian generators of the gauge group $G_{\mathrm{YM}}$. The gauge connection $\boldsymbol{V}_{\hat{A}}$ and the field strengths $\boldsymbol{F}_{\hat{A} \hat{B}}$ and $\boldsymbol{W}$ can be decomposed as $\boldsymbol{V}_{\hat{A}}=V_{\hat{A}}^{I} T_{I}, \boldsymbol{F}_{\hat{A} \hat{B}}=$ $F_{\hat{A} \hat{B}}{ }^{I} T_{I}$ and $\boldsymbol{W}=W^{I} T_{I}$. For a single abelian vector multiplet, we will use $V_{\hat{A}}, F_{\hat{A} \hat{B}}$ and $W$.

It is helpful to introduce the following descendant superfields constructed from spinor derivatives of $\boldsymbol{W}$ :

$$
\boldsymbol{\lambda}_{\hat{\alpha}}^{i}:=-\mathrm{i} \boldsymbol{\nabla}_{\hat{\alpha}}^{i} \boldsymbol{W}, \quad \boldsymbol{X}^{i j}:=\frac{\mathrm{i}}{4} \boldsymbol{\nabla}^{\hat{\alpha}(i} \nabla_{\hat{\alpha}}^{j)} \boldsymbol{W}=-\frac{1}{4} \boldsymbol{\nabla}^{\hat{\alpha}(i} \boldsymbol{\lambda}_{\hat{\alpha}}^{j)} .
$$

The above superfields together with

$$
\boldsymbol{F}_{\hat{\alpha} \hat{\beta}}=-\frac{\mathrm{i}}{4} \nabla_{(\hat{\alpha}}^{k} \nabla_{\hat{\beta}) k} \boldsymbol{W}-W_{\hat{\alpha} \hat{\beta}} \boldsymbol{W}=\frac{1}{4} \boldsymbol{\nabla}_{(\hat{\alpha}}^{k} \boldsymbol{\lambda}_{\hat{\beta}) k}-W_{\hat{\alpha} \hat{\beta}} \boldsymbol{W}
$$

satisfy the following useful identities:

$$
\begin{aligned}
\boldsymbol{\nabla}_{\hat{\alpha}}^{i} \boldsymbol{\lambda}_{\hat{\beta}}^{j} & =-2 \varepsilon^{i j}\left(\boldsymbol{F}_{\hat{\alpha} \hat{\beta}}+W_{\hat{\alpha} \hat{\beta}} \boldsymbol{W}\right)-\varepsilon_{\hat{\alpha} \hat{\beta}} \boldsymbol{X}^{i j}-\varepsilon^{i j} \boldsymbol{\nabla}_{\hat{\alpha} \hat{\beta}} \boldsymbol{W}, \\
\boldsymbol{\nabla}_{\hat{\alpha}}^{i} \boldsymbol{F}_{\hat{\beta} \hat{\gamma}} & =-\mathrm{i} \boldsymbol{\nabla}_{\hat{\alpha}(\hat{\beta}} \boldsymbol{\lambda}_{\hat{\gamma})}^{i}-\mathrm{i} \varepsilon_{\hat{\alpha}(\hat{\beta}} \boldsymbol{\nabla}_{\hat{\gamma}}{ }_{\hat{\delta}} \boldsymbol{\lambda}_{\hat{\delta}}^{i}-\mathrm{i} W_{\hat{\beta} \hat{\gamma}} \boldsymbol{\lambda}_{\hat{\alpha}}^{i}-W_{\hat{\alpha} \hat{\beta} \hat{\gamma}}^{i} \boldsymbol{W}-\varepsilon_{\hat{\alpha}(\hat{\beta}} X_{\hat{\gamma})}^{i} \boldsymbol{W}, \\
\boldsymbol{\nabla}_{\hat{\alpha}}^{i} \boldsymbol{X}^{j k} & =2 \mathrm{i} \varepsilon^{i(j}\left(\boldsymbol{\nabla}_{\hat{\alpha}}^{\hat{\beta}} \boldsymbol{\lambda}_{\hat{\beta}}^{k)}+W_{\hat{\alpha} \hat{\beta}} \boldsymbol{\lambda}^{\hat{\beta} k)}-\frac{\mathrm{i}}{2} X_{\hat{\alpha}}^{k)} \boldsymbol{W}-\mathrm{i}\left[\boldsymbol{W}, \boldsymbol{\lambda}_{\hat{\alpha}}^{k)}\right]\right) .
\end{aligned}
$$

The $S$-supersymmetry generator acts on these descendants as

$$
S_{\hat{\alpha}}^{i} \boldsymbol{\lambda}_{\hat{\beta}}^{j}=-2 \mathrm{i} \varepsilon_{\hat{\alpha} \hat{\beta}} \varepsilon^{i j} \boldsymbol{W}, \quad S_{\hat{\alpha}}^{i} \boldsymbol{F}_{\hat{\beta} \hat{\gamma}}=4 \varepsilon_{\hat{\alpha}(\hat{\beta}} \boldsymbol{\lambda}_{\hat{\gamma})}^{i}, \quad S_{\hat{\alpha}}^{i} \boldsymbol{X}^{j k}=-2 \varepsilon^{i(j} \boldsymbol{\lambda}_{\hat{\alpha}}^{k)} .
$$

Now consider a primary composite superfield $\boldsymbol{H}_{\mathrm{YM}}^{i j}$ that is quadratic in the generators of the gauge group and is defined by

$$
\begin{aligned}
\boldsymbol{H}_{\mathrm{YM}}^{i j} & =\mathrm{i}\left(\boldsymbol{\nabla}^{\hat{\alpha}(i} \boldsymbol{W}\right) \boldsymbol{\nabla}_{\hat{\alpha}}^{j)} \boldsymbol{W}+\frac{\mathrm{i}}{4}\left\{\boldsymbol{W}, \boldsymbol{\nabla}^{\hat{\alpha}(i} \nabla_{\hat{\alpha}}^{j)} \boldsymbol{W}\right\} \\
& =\left\{\boldsymbol{W}, \boldsymbol{X}^{i j}\right\}-\mathrm{i} \boldsymbol{\lambda}^{\hat{\alpha}(i} \boldsymbol{\lambda}_{\hat{\alpha}}^{j)} .
\end{aligned}
$$


Its important property is

$$
\boldsymbol{\nabla}_{\hat{\alpha}}^{(i} \boldsymbol{H}_{\mathrm{YM}}^{j k)}=0 .
$$

In the rigid superspace limit, $\boldsymbol{H}_{\mathrm{YM}}^{i j}$ reduces to the composite superfield introduced in [2]. Associated with $\boldsymbol{H}_{\mathrm{YM}}^{i j}$ is the gauge singlet $H_{\mathrm{YM}}^{i j}:=\operatorname{tr} \boldsymbol{H}_{\mathrm{YM}}^{i j}$, which is a primary superfield constrained by $\nabla_{\hat{\alpha}}^{(i} H_{\mathrm{YM}}^{j k)}=0$. This is an example of a covariant $\mathcal{O}(2)$ multiplet defined in section 5 .

\section{From conformal to SU(2) superspace}

The superspace structure we have presented in the previous section involves, as in four and three dimensions [38-40], the gauging of the entire superconformal algebra in order to describe conformal supergravity. Traditionally, however, conformal supergravity has been described in superspace in a different manner: local component scale and special conformal transformations were encoded in super Weyl transformations. This was exactly the approach taken previously in [26] where 5D conformal supergravity was described by gauging $\mathrm{SO}(4,1) \times \mathrm{SU}(2)$, corresponding to the Lorentz and $R$-symmetry groups, with additional super Weyl transformations realized non-linearly. As in the introduction, we refer to the latter formulation of conformal supergravity as $\mathrm{SU}(2)$ superspace.

The relation between these two approaches mirrors the simpler non-supersymmetric situation. Conformal gravity may be described as the gauge theory of the conformal algebra, with a vielbein, Lorentz, dilatation, and special conformal connection. Certain constraints are usually imposed so that the only independent fields are the vielbein and dilatation connection. A special conformal transformation can be made to eliminate the dilatation connection; upon making such a choice, one keeps the vielbein and Lorentz connections in the covariant derivative, while discarding the special conformal connection this is often called "degauging" the special conformal symmetry. The dilatation symmetry survives as the usual Weyl symmetry of the vielbein, and one recovers a formulation of conformal gravity with a vielbein alone.

As alluded to in the introduction, it is possible to "degauge" conformal superspace to recover $\mathrm{SU}(2)$ superspace in a similar way. This is the goal of this section. The procedure follows exactly the path laid out in the four and three dimensional cases [38-40]. In particular, we will show explicitly how to recover the connections and curvatures of SU(2) superspace and derive the form of the super Weyl transformations. The material in this section provides the necessary ingredients to relate results in conformal superspace to those of $\mathrm{SU}(2)$ superspace.

\subsection{Degauging to $\mathrm{SU}(2)$ superspace}

Let us recall that $\mathrm{SU}(2)$ superspace is described by a superspace vielbein, Lorentz connection, and $\mathrm{SU}(2)_{\mathrm{R}}$ connection. Conformal superspace possesses in addition dilatation and special conformal connections; these must be dealt with in a particular way. The first step is to eliminate the dilatation connection. Because the one-form $B=E^{\hat{a}} B_{\hat{a}}+E_{i}^{\hat{\alpha}} B_{\hat{\alpha}}^{i}$ transforms as

$$
\delta_{K}(\Lambda) B=-2 E^{\hat{a}} \Lambda_{\hat{a}}-2 E_{i}^{\hat{\alpha}} \Lambda_{\hat{\alpha}}^{i},
$$


under special conformal transformations, it is straightforward to impose the gauge choice

$$
B_{\hat{A}}=0,
$$

eliminating the dilatation connection entirely. The special conformal connection $\mathfrak{F}^{\hat{A}}$ remains, but its corresponding gauge symmetry has been fixed, so we will extract it from the covariant derivative. The resulting degauged covariant derivatives are given by

$$
\mathcal{D}_{\hat{A}}:=\nabla_{\hat{A}}+\mathfrak{F}_{\hat{A}}^{\hat{B}} K_{\hat{B}}=E_{\hat{A}}-\frac{1}{2} \Omega_{\hat{A}}^{\hat{b} \hat{c}} M_{\hat{b} \hat{c}}-\Phi_{\hat{A}}^{i j} J_{i j},
$$

and possess an $\mathrm{SO}(4,1) \times \mathrm{SU}(2)$ structure group. They satisfy (anti-)commutation relations of the form ${ }^{6}$

$$
\left[\mathcal{D}_{\hat{A}}, \mathcal{D}_{\hat{B}}\right\}=-\widetilde{\mathscr{T}}_{\hat{A} \hat{B}} \hat{C} \mathcal{D}_{\hat{C}}-\frac{1}{2} \widetilde{\mathscr{R}}_{\hat{A} \hat{B}}^{\hat{c} \hat{d}} M_{\hat{c} \hat{d}}-\widetilde{\mathscr{R}}_{\hat{A} \hat{B}}^{k l} J_{k l} .
$$

Because the vielbein, Lorentz, and SU(2) connections are exactly those of conformal superspace, it is easy to give expressions for the new torsion and curvature tensors in terms of the conformal ones using (2.26). For example, one finds for the torsion tensor,

$$
\widetilde{\mathscr{T}}^{\hat{a}}=\mathscr{T}^{\hat{a}}, \quad \widetilde{\mathscr{T}}_{i}^{\hat{\alpha}}=\mathscr{T}_{i}^{\hat{\alpha}}+\mathrm{i} E^{\hat{c}} \wedge \mathfrak{F}_{i}^{\hat{\beta}}\left(\Gamma_{\hat{c}}\right)_{\hat{\beta}}^{\hat{\alpha}} .
$$

The special conformal connections $\mathfrak{F}_{\hat{A}}{ }^{\hat{B}}$ provide new contributions to the superfield torsion and similarly to the other curvatures.

It turns out there is actually a subtlety in this degauging procedure. A careful examination of (3.5) shows that one recovers almost all the same constraints on the torsion tensor as in $\mathrm{SU}(2)$ superspace, except that

$$
\widetilde{\mathscr{T}}_{\hat{a}} \hat{\beta}_{(j k)}^{\hat{\beta}} \neq 0, \quad \widetilde{\mathscr{T}}_{\hat{a} \hat{b}}^{\hat{c}} \neq 0 .
$$

In $\mathrm{SU}(2)$ superspace, both of these combinations are required to vanish. The solution to this is that there is some freedom to redefine the vector components of the Lorentz and $\mathrm{SU}(2)$ connections when we degauge, corresponding to a redefinition of the vector covariant derivative of $\mathrm{SU}(2)$ superspace. This in turn modifies the torsion and curvature tensors. A particular choice sets to zero the combinations (3.6) and exactly reproduces the torsion and curvature tensors of $\mathrm{SU}(2)$ superspace. To elaborate further, we must analyze explicitly the additional superfields introduced by the special conformal connections $\mathfrak{F}_{\hat{A}}{ }_{\hat{B}}$.

\subsection{The degauged special conformal connection}

In the gauge (3.2) the dilatation curvature is given by ${ }^{7}$

$$
\mathscr{R}(\mathbb{D})_{\hat{A} \hat{B}}=2 \mathfrak{F}_{\hat{A} \hat{B}}(-1)^{\varepsilon_{\hat{B}}}-2 \mathfrak{F}_{\hat{B} \hat{A}}(-1)^{\varepsilon_{\hat{A}}+\varepsilon_{\hat{A}} \varepsilon_{\hat{B}}}
$$

The vanishing of the dilatation curvature at dimension- 1 constrains the special conformal connection as ${ }^{8}$

$$
\mathfrak{F}_{\hat{\alpha} \hat{\beta}}^{i j}=-\mathfrak{F}_{\hat{\beta} \hat{\alpha}}^{j i}=\frac{\mathrm{i}}{2} \varepsilon_{\hat{\alpha} \hat{\beta}} S^{i j}-\frac{\mathrm{i}}{4} C_{\hat{\alpha} \hat{\beta}}^{i j}+\mathrm{i} \varepsilon^{i j} Y_{\hat{\alpha} \hat{\beta}},
$$

\footnotetext{
${ }^{6}$ We distinguish the degauged versions of the torsion and curvatures with a tilde.

${ }^{7}$ We have lowered the index on the $K$-connection as $\mathfrak{F}_{\hat{A} \hat{b}}=\eta_{\hat{b} \hat{c}} \mathfrak{F}_{\hat{A}}^{\hat{c}}$ and $\mathfrak{F}_{\hat{A} \hat{\beta}}^{j}=\varepsilon_{\hat{\beta} \hat{\gamma}} \mathfrak{F}_{\hat{A}} \hat{\gamma}^{j}$.

${ }^{8}$ The reason for introducing these superfields via these coefficients will be clear later.
} 
where the superfields $S^{i j}, C_{\hat{\alpha} \hat{\beta}}^{i j}, Y_{\hat{\alpha} \hat{\beta}}$ satisfy the symmetry properties

$$
S^{i j}=S^{j i}, \quad C_{\hat{\alpha} \hat{\beta}}^{i j}=\left(\Gamma^{\hat{a}}\right)_{\hat{\alpha} \hat{\beta}} C_{\hat{a}}^{i j}=C_{\hat{\alpha} \hat{\beta}}^{j i}, \quad Y_{\hat{\alpha} \hat{\beta}}=Y_{\hat{\beta} \hat{\alpha}} .
$$

From here it is possible to derive the degauged covariant derivative algebra by computing $\left[\mathcal{D}_{\hat{A}}, \mathcal{D}_{\hat{B}}\right\}$. An efficient way to do this is to consider a primary superfield $\Phi$ transforming as a tensor in some representation of the remainder of the superconformal algebra (compare with [39]). For example, to determine the anti-commutator of spinor derivatives we consider

$$
\left.\left\{\mathcal{D}_{\hat{\alpha}}^{i}, \mathcal{D}_{\hat{\beta}}^{j}\right\} \Phi=\left\{\nabla_{\hat{\alpha}}^{i}, \nabla_{\hat{\beta}}^{j}\right\} \Phi+\mathfrak{F}_{\hat{\alpha}}^{i \hat{C}}\left[K_{\hat{C}}, \nabla_{\hat{\beta}}^{j}\right\} \Phi+\mathfrak{F}_{\hat{\beta}}^{j} \hat{C}_{\left[K_{\hat{C}}\right.}, \nabla_{\hat{\alpha}}^{i}\right\} \Phi .
$$

Making use of the form of $\mathfrak{F}$ and of the superconformal algebra we find

$$
\begin{aligned}
\left\{\mathcal{D}_{\hat{\alpha}}^{i}, \mathcal{D}_{\hat{\beta}}^{j}\right\}= & -2 \mathrm{i} \varepsilon^{i j} \mathcal{D}_{\hat{\alpha} \hat{\beta}}^{\prime}+3 \mathrm{i} \varepsilon_{\hat{\alpha} \hat{\beta}} \varepsilon^{i j} S^{k l} J_{k l}-\mathrm{i} \varepsilon^{i j} C_{\hat{\alpha} \hat{\beta}}^{k l} J_{k l}-12 \mathrm{i} Y_{\hat{\alpha} \hat{\beta}} J^{i j} \\
& -\mathrm{i} \varepsilon_{\hat{\alpha} \hat{\beta}} \varepsilon^{i j}\left(W^{\hat{c} \hat{d}}+Y^{\hat{c} \hat{d}}\right) M_{\hat{c} \hat{d}}+\frac{\mathrm{i}}{4} \varepsilon^{i j} \varepsilon^{\hat{a} \hat{b} \hat{c} \hat{d} \hat{e}}\left(\Gamma_{\hat{a}}\right)_{\hat{\alpha} \hat{\beta}}\left(2 Y_{\hat{b} \hat{c}}-W_{\hat{b} \hat{c}}\right) M_{\hat{d} \hat{e}} \\
& -\frac{\mathrm{i}}{2} \varepsilon^{\hat{a} \hat{b} \hat{c} \hat{d} \hat{e}}\left(\Sigma_{\hat{a} \hat{b}}\right)_{\hat{\alpha} \hat{\beta}} C_{\hat{c}}^{i j} M_{\hat{d} \hat{e}}+4 \mathrm{i} S^{i j} M_{\hat{\alpha} \hat{\beta}},
\end{aligned}
$$

where we have defined the vector covariant derivative

$$
\mathcal{D}_{\hat{a}}^{\prime}:=\mathcal{D}_{\hat{a}}+\frac{1}{4} C_{\hat{a}}^{k l} J_{k l}-\frac{1}{8} \varepsilon_{\hat{a} \hat{b} \hat{c} \hat{d} \hat{e}} W^{\hat{b} \hat{c}} M^{\hat{d} \hat{e}} .
$$

The remaining algebra of covariant derivatives can be similarly computed directly from degauging. It can be seen that the algebra of $\mathcal{D}_{\hat{A}}^{\prime}=\left(\mathcal{D}_{\hat{a}}^{\prime}, \mathcal{D}_{\hat{\alpha}}^{i}\right)$ exactly matches the one of $\mathrm{SU}(2)$ superspace [26] once we identify the dimension-1 torsion components $X_{\hat{a} \hat{b}}$ and $N_{\hat{a} \hat{b}}$ used in [26] as

$$
X_{\hat{a} \hat{b}}:=W_{\hat{a} \hat{b}}+Y_{\hat{a} \hat{b}}, \quad N_{\hat{a} \hat{b}}:=2 Y_{\hat{a} \hat{b}}-W_{\hat{a} \hat{b}} .
$$

The superfields $S^{i j}$ and $C_{\hat{a}}{ }^{i j}$, which we introduced in (3.8), are equivalent to the ones used in [26]. In particular, it turns out that the covariant derivative algebra for $\mathcal{D}_{\hat{A}}^{\prime}$ does not possess the torsion components (3.6).

The curvature superfields can be shown to satisfy the dimension-3/2 identities:

$$
\begin{aligned}
& \mathcal{D}_{\hat{\gamma}}^{k} W_{\hat{a} \hat{b}}=W_{\hat{a} \hat{b} \hat{\gamma}}{ }^{k}+\left(\Sigma_{\hat{a} \hat{b}}\right) \hat{\gamma}{ }_{\hat{\gamma}}^{\hat{\delta}} X_{\hat{\delta}}^{k}, \\
& \mathcal{D}_{\hat{\gamma}}^{k} Y_{\hat{a} \hat{b}}=2\left(\Gamma_{[\hat{a}}\right) \hat{\gamma}_{\hat{\gamma}}^{\hat{\delta}} \mathcal{Y}_{\hat{b}] \hat{\delta}}^{k}+\left(\Sigma_{\hat{a} \hat{b}}\right) \hat{\gamma}^{\hat{\delta}} \mathcal{Y}_{\hat{\delta}}^{k} \text {, } \\
& \mathcal{D}_{\hat{\gamma}}^{k} C_{\hat{a}}^{i j}=-\frac{1}{2}\left(\Gamma_{\hat{a}}\right)_{\hat{\gamma}}^{\hat{\delta}} \mathcal{C}_{\hat{\delta}}^{i j k}-\frac{2}{3}\left(\mathcal{C}_{\hat{a} \hat{\gamma}}^{(i}-\frac{1}{2}\left(\Gamma_{\hat{a}}\right)_{\hat{\gamma}}^{\hat{\delta}} \mathcal{C}_{\hat{\delta}}^{(i)}\right) \varepsilon^{j) k} \text {, } \\
& \mathcal{D}_{\hat{\gamma}}^{k} S^{i j}=-\frac{1}{4} \mathcal{C}_{\hat{\gamma}}^{i j k}+\left(X_{\hat{\gamma}}^{(i}+\frac{5}{2} \mathcal{Y}_{\hat{\gamma}}^{(i}+\frac{5}{12} \mathcal{C}_{\hat{\gamma}}^{(i}\right) \varepsilon^{j) k},
\end{aligned}
$$

where

$$
\left(\Gamma^{\hat{a}}\right)_{\hat{\alpha}}^{\hat{\beta}} W_{\hat{a} \hat{b} \hat{\beta}}^{i}=0, \quad\left(\Gamma^{\hat{a}}\right)_{\hat{\alpha}}^{\hat{\beta}} \mathcal{Y}_{\hat{a} \hat{\beta}}^{i}=0, \quad\left(\Gamma^{\hat{a}}\right)_{\hat{\alpha}}^{\hat{\beta}} \mathcal{C}_{\hat{a} \hat{\beta}}^{i}=0, \quad \mathcal{C}_{\hat{\alpha}}^{i j k}=\mathcal{C}_{\hat{\alpha}}^{(i j k)}
$$

Note that the dimension- $3 / 2$ torsion is

$$
\widetilde{\mathscr{T}}_{\hat{a} \hat{b} \hat{\gamma}}^{k}=\frac{\mathrm{i}}{2} \mathcal{D}_{\hat{\gamma}}^{k} W_{\hat{a} \hat{b}}+\frac{\mathrm{i}}{2} \mathcal{D}_{\hat{\gamma}}^{k} Y_{\hat{a} \hat{b}}-\frac{\mathrm{i}}{6}\left(\Gamma_{[\hat{a}}\right)_{\hat{\gamma}}^{\hat{\delta}} \mathcal{C}_{\hat{b}] \hat{\delta}}^{k}+\frac{\mathrm{i}}{4}\left(\Sigma_{\hat{a} \hat{b}}\right) \hat{\gamma}_{\hat{\gamma}}^{\hat{\delta}} \mathcal{C}_{\hat{\delta}}^{k}
$$


To degauge results in conformal superspace it is useful to also have the remaining special conformal connection components $\mathfrak{F}_{\hat{a}}{ }^{\hat{\beta} j}$ and $\mathfrak{F}_{\hat{a} \hat{b}}$. They are constrained by the dilatation curvature as follows: ${ }^{9}$

$$
\begin{aligned}
&-\frac{1}{5}\left(\Gamma_{\hat{a}}\right)_{\hat{\beta}} \hat{\gamma} \mathcal{D}^{\hat{\delta} j} W_{\hat{\gamma} \hat{\delta}}=-2 \mathfrak{F}_{\hat{a}}^{j}-2 \mathfrak{F}_{\hat{\beta}}^{j} \hat{a}, \\
&-\frac{1}{2} \varepsilon_{\hat{a} \hat{b} \hat{c} \hat{d} \hat{e}} \mathcal{D}^{\hat{c}} W^{\hat{d} \hat{e}}=4 \mathfrak{F}_{[\hat{a} \hat{b}]} .
\end{aligned}
$$

The explicit expressions for $\mathfrak{F}_{\hat{a}}^{j}{ }_{\hat{\beta}}^{j}$ and $\mathfrak{F}_{\hat{a} \hat{b}}$ may be found by analyzing the special conformal curvatures

$$
\begin{aligned}
& \mathscr{R}(S)_{\hat{A} \hat{B}}^{\hat{\gamma} k}=2 \mathcal{D}_{[\hat{A}} \widetilde{F}_{\hat{B}\}}{ }^{\hat{\gamma} k}+\widetilde{\mathscr{T}}_{\hat{A} \hat{B}} \hat{D}_{\mathfrak{F}_{\hat{D}}}{ }^{\hat{\gamma} k}+\mathrm{i} \delta_{\hat{A}} \hat{\delta}^{\hat{\delta}} \mathfrak{F}_{\hat{B}}{ }^{\hat{c}}\left(\Gamma_{\hat{c}}\right)_{\hat{\delta}} \hat{\gamma}(-1)^{\varepsilon_{\hat{B}}} \\
& -\mathrm{i} \hat{\delta}_{\hat{B}}^{\hat{\delta} k} \mathfrak{F}_{\hat{A}}^{\hat{c}}\left(\Gamma_{\hat{c}}\right)_{\hat{\delta}}^{\hat{\gamma}}(-1)^{\varepsilon_{\hat{B}} \varepsilon_{\hat{A}}+\varepsilon_{\hat{A}}}, \\
& \mathscr{R}(K)_{\hat{A} \hat{B}}^{\hat{c}}=2 \mathcal{D}_{[\hat{A}} \mathfrak{F}_{\hat{B}\}}{ }^{\hat{c}}+\widetilde{\mathscr{T}}_{\hat{A} \hat{B}} \hat{D}_{\mathfrak{F}_{\hat{D}}}^{\hat{c}}+\mathrm{i} \mathfrak{F}_{\hat{A} k} \hat{\gamma} \mathfrak{F}_{\hat{B}}{ }^{\hat{\delta} k}\left(\Gamma^{\hat{c}}\right)_{\hat{\gamma} \hat{\delta}}(-1)^{\varepsilon_{\hat{B}}} \\
& -\mathrm{i} \mathfrak{F}_{\hat{B} k} \hat{\gamma}_{\mathfrak{A}} \mathfrak{F}_{\hat{A}}^{\hat{\delta} k}\left(\Gamma^{\hat{c}}\right)_{\hat{\gamma} \hat{\delta}}(-1)^{\varepsilon_{\hat{B}^{-}} \hat{A}_{\hat{A}}+\varepsilon_{\hat{A}}}
\end{aligned}
$$

which appear in the algebra of the conformal covariant derivatives $\nabla_{\hat{A}}$. The component special conformal connections are given by:

$$
\begin{aligned}
& \mathfrak{F}_{\hat{a}}^{j}{ }_{\hat{\beta}}^{j}=-\frac{1}{10}\left(\Gamma_{\hat{a}}\right)_{\hat{\beta}} \hat{\mathcal{D}}_{\hat{\gamma} k} S^{j k}-\frac{1}{18}\left(\Sigma_{\hat{a} \hat{b}}\right)_{\hat{\beta}}^{\hat{\gamma}} \mathcal{D}_{\hat{\gamma} k} C^{\hat{b} j k}-\frac{1}{24} \varepsilon_{\hat{a} \hat{b} \hat{c} \hat{d} \hat{e}}\left(\Sigma^{\hat{d} \hat{e}}\right)_{\hat{\beta}}{ }^{\hat{\gamma}} \mathcal{D}_{\hat{\gamma}}^{j} Y^{\hat{b} \hat{c}} \\
& -\frac{1}{12}\left(\Gamma^{\hat{b}}\right)_{\hat{\beta}}^{\hat{\gamma}} \mathcal{D}_{\hat{\gamma}}^{j} Y_{\hat{a} \hat{b}}+\frac{1}{18} \mathcal{D}_{\hat{\beta} k} C_{\hat{a}}^{j k}+\frac{1}{30}\left(\Gamma_{\hat{a}}\right)_{\hat{\beta}}{ }^{\hat{\gamma}} \mathcal{D}^{\hat{\delta} j} W_{\hat{\gamma} \hat{\delta}} \\
& =\frac{1}{2} \mathcal{Y}_{\hat{a}}^{j}{ }_{\hat{\beta}}-\frac{1}{12} \mathcal{C}_{\hat{a}}^{j}{ }_{\hat{\beta}}^{j}-\frac{1}{16}\left(\Gamma_{\hat{a}}\right)_{\hat{\beta}} \hat{\mathcal{C}}_{\hat{\gamma}}^{j}-\frac{1}{8}\left(\Gamma_{\hat{a}}\right)_{\hat{\beta}} \hat{\gamma} \mathcal{Y}_{\hat{\gamma}}^{j}, \\
& \mathfrak{F}_{\hat{\beta}}^{j} \hat{a}^{\hat{a}}=-\mathfrak{F}_{\hat{a}_{\hat{\beta}}}^{j}+\frac{1}{10}\left(\Gamma_{\hat{a}}\right)_{\hat{\beta}}{ }_{\hat{\gamma}} \mathcal{D}^{\hat{\delta} j} W_{\hat{\gamma} \hat{\delta}}=-\mathfrak{F}_{\hat{a}_{\hat{\beta}}}^{j}+\frac{1}{4}\left(\Gamma_{\hat{a}}\right)_{\hat{\beta}} \hat{\gamma} X_{\hat{\gamma}}^{j}, \\
& \mathfrak{F}_{\hat{a} \hat{b}}=\frac{\mathrm{i}}{288} \eta_{\hat{a} \hat{b}}\left[\mathcal{D}^{\hat{\alpha} i}, \mathcal{D}_{\hat{\alpha}}^{j}\right] S_{i j}+\frac{\mathrm{i}}{576} \eta_{\hat{a} \hat{b}}\left[\mathcal{D}_{i}^{\hat{\alpha}}, \mathcal{D}_{j}^{\hat{\beta}}\right] C_{\hat{\alpha} \hat{\beta}}{ }^{i j}-\frac{\mathrm{i}}{128}\left(\Gamma_{(\hat{a}}\right)^{\hat{\alpha} \hat{\beta}}\left[\mathcal{D}_{\hat{\alpha}}^{i}, \mathcal{D}_{\hat{\beta}}^{j}\right] C_{\hat{b})^{i j}} \\
& -\frac{\mathrm{i}}{96} \eta_{\hat{a} \hat{b}}\left[\mathcal{D}_{\hat{\alpha}}^{k}, \mathcal{D}_{\hat{\beta} k}\right] Y^{\hat{\alpha} \hat{\beta}}-\frac{\mathrm{i}}{48}\left(\Sigma^{\hat{c}}{ }_{(\hat{a}}\right)^{\hat{\alpha} \hat{\beta}}\left[\mathcal{D}_{\hat{\alpha}}^{k}, \mathcal{D}_{\hat{\beta} k}\right] Y_{\hat{b}) \hat{c}}+\frac{\mathrm{i}}{240} \eta_{\hat{a} \hat{b}}\left[\mathcal{D}_{\hat{\alpha}}^{k}, \mathcal{D}_{\hat{\beta} k}\right] W^{\hat{\alpha} \hat{\beta}} \\
& -\frac{1}{8} \eta_{\hat{a} \hat{b}} S^{k l} S_{k l}+\frac{1}{16} C_{\hat{a}}^{k l} C_{\hat{b} k l}-\frac{1}{32} \eta_{\hat{a} \hat{b}} C^{\hat{c} k l} C_{\hat{c} k l} \\
& +\frac{1}{2} Y_{(\hat{a}}^{\hat{c}} Y_{\hat{b}) \hat{c}}-\frac{1}{8} \eta_{\hat{a} \hat{b}} Y^{\hat{c} \hat{d}} Y_{\hat{c} \hat{d}}-\frac{1}{8} \varepsilon_{\hat{a} \hat{b} \hat{c} \hat{d} \hat{e}} \mathcal{D}^{\hat{c}} W^{\hat{d} \hat{e}} \text {. }
\end{aligned}
$$

The above results provide us with the ingredients needed to degauge conformal superspace to $\mathrm{SU}(2)$ superspace. For example, one finds the commutator

$$
\begin{aligned}
{\left[\mathcal{D}_{\hat{a}}^{\prime}, \mathcal{D}_{\hat{\beta}}^{j}\right]=- } & \frac{1}{2}\left[\left(Y_{\hat{a} \hat{b}}+W_{\hat{a} \hat{b}}\right)\left(\Gamma^{\hat{b}}\right)_{\hat{\beta}}{ }_{\hat{\gamma}} \delta_{k}^{j}+\frac{1}{4} \varepsilon_{\hat{a} \hat{b} \hat{c} \hat{d} \hat{e}}\left(2 Y^{\hat{d} \hat{e}}-W^{\hat{d} \hat{e}}\right)\left(\Sigma^{\hat{b} \hat{c}}\right)_{\hat{\beta}}{ }^{\hat{\gamma}} \delta_{k}^{j}\right. \\
& \left.-\left(\Gamma_{\hat{a}}\right)_{\hat{\beta}}{ }^{\hat{\gamma}} S^{j}{ }_{k}-\left(\Sigma_{\hat{a} \hat{b}}\right)_{\hat{\beta}}^{\hat{\gamma}} C^{\hat{b} j}{ }_{k}\right] \mathcal{D}_{\hat{\gamma}}^{k} \\
+ & \frac{1}{2}\left[\left(\Gamma_{\hat{a}}\right)_{\hat{\beta}}{ }_{\hat{\gamma}} W^{\hat{c} \hat{d} k}{ }_{\hat{\gamma}}+\delta_{\hat{a}}^{[\hat{c}}\left(\frac{1}{3} \mathcal{C}^{\hat{d}]}{ }_{\hat{\beta}}^{j}-2 \mathcal{Y}^{\hat{d}]}{ }_{\hat{\beta}}^{j}+\frac{1}{2}\left(\Gamma^{\hat{d}]}\right)_{\hat{\beta}}^{\hat{\gamma}}\left(\mathcal{C}_{\hat{\gamma}}^{j}+2 \mathcal{Y}_{\hat{\gamma}}^{j}+2 X_{\hat{\gamma}}^{j}\right)\right)\right.
\end{aligned}
$$

\footnotetext{
${ }^{9}$ Here we raise and lower the indices on the special conformal connection using $\varepsilon^{i j}, \varepsilon_{\hat{\alpha} \hat{\beta}}$ and $\eta_{\hat{a} \hat{b}}$ in the usual way.
} 


$$
\begin{aligned}
& \left.+\left(\Sigma^{\hat{c} \hat{d}}\right)_{\hat{\beta}}^{\hat{\gamma}}\left(2 \mathcal{Y}_{\hat{a} \hat{\gamma}}^{j}-\frac{1}{3} \mathcal{C}_{\hat{a} \hat{\gamma}}^{j}\right)+\frac{1}{8} \varepsilon_{\hat{a}}^{\hat{c} \hat{d} \hat{e} \hat{f}}\left(\Sigma_{\hat{e} \hat{f}}\right)_{\hat{\beta}} \hat{\gamma}\left(\mathcal{C}_{\hat{\gamma}}^{j}+2 \mathcal{Y}_{\hat{\gamma}}^{j}\right)\right] M_{\hat{c} \hat{d}} \\
& +\left[3 \mathcal{Y}_{\hat{a}}^{(k} \varepsilon^{\varepsilon l) j}-\frac{1}{3} \mathcal{C}_{\hat{a}}^{(k} \varepsilon^{l) j}+\frac{1}{8}\left(\Gamma_{\hat{a}}\right)_{\hat{\beta}}{ }^{\hat{\gamma}} \mathcal{C}_{\hat{\gamma}}^{j k l}-\frac{11}{24}\left(\Gamma_{\hat{a}}\right)_{\hat{\beta}}{ }^{\hat{\gamma}} \mathcal{C}_{\hat{\gamma}}^{(k} \varepsilon^{l) j}\right. \\
& \left.-\frac{3}{4}\left(\Gamma_{\hat{a}}\right)_{\hat{\beta}}^{\hat{\gamma}}\left(\mathcal{Y}_{\hat{\gamma}}^{(k}+2 X_{\hat{\gamma}}^{(k)}\right) \varepsilon^{l) j}\right] J_{k l},
\end{aligned}
$$

which agrees with [26] up to field redefinitions. One can also derive the $\left[\mathcal{D}_{\hat{a}}^{\prime}, \mathcal{D}_{\hat{b}}^{\prime}\right]$ commutator, which we will not need for this paper.

\subsection{The conformal origin of the super Weyl transformations}

We have just shown that $\mathrm{SU}(2)$ superspace is a degauged version of conformal superspace, in which the dilatation connection is gauged away. Although the dilatations and special conformal transformations are not manifestly realized, the dilatation symmetry has not been fixed. The symmetry remains as additional nonlinear transformations, known as super Weyl transformations. Their presence in $\mathrm{SU}(2)$ superspace ensures that it describes conformal supergravity. Below we show how to recover the super Weyl transformations from the degauging of conformal superspace.

Suppose we have gauge fixed the dilatation connection to vanish by using the special conformal symmetry. If we now perform a dilatation with parameter $\sigma$, we must accompany it with an additional $K_{\hat{A}}$ transformation with $\sigma$-dependent parameters $\Lambda^{\hat{A}}(\sigma)$ to maintain the gauge $B_{\hat{A}}=0$, which requires

$$
\left(\delta_{K}(\Lambda(\sigma))+\delta_{\mathbb{D}}(\sigma)\right) B_{\hat{A}}=0 .
$$

Using the transformation rule $(2.21)$, we find

$$
\Lambda^{\hat{a}}(\sigma)=\frac{1}{2} \mathcal{D}^{\hat{a}} \sigma, \quad \Lambda^{\hat{\alpha} i}(\sigma)=-\frac{1}{2} \mathcal{D}^{\hat{\alpha} i} \sigma .
$$

Note that all primary superfields $\Phi$ transform homogeneously

$$
\delta_{K}(\Lambda(\sigma)) \Phi+\delta_{\mathbb{D}}(\sigma) \Phi=\delta_{\mathbb{D}}(\sigma) \Phi=w \sigma \Phi,
$$

where $w$ is the dimension of $\Phi, \mathbb{D} \Phi=w \Phi$. For example, the super Weyl tensor transforms as

$$
\delta_{\sigma} W_{\hat{\alpha} \hat{\beta}}=\sigma W_{\hat{\alpha} \hat{\beta}} .
$$

The super Weyl transformations of the degauged covariant derivatives $\mathcal{D}_{\hat{A}}$ and the special conformal connection can be read from

$$
\delta_{\sigma} \nabla_{\hat{A}}=\delta_{\sigma} \mathcal{D}_{\hat{A}}-\delta_{\sigma} \mathfrak{F}_{\hat{A}}^{\hat{B}} K_{\hat{B}}=\delta_{K}(\Lambda(\sigma)) \nabla_{\hat{A}}+\delta_{\mathbb{D}}(\sigma) \nabla_{\hat{A}},
$$

implying that the super Weyl transformations of $\mathcal{D}_{\hat{A}}$ are

$$
\begin{aligned}
& \delta_{\sigma} \mathcal{D}_{\hat{\alpha}}^{i}=\frac{1}{2} \sigma \mathcal{D}_{\hat{\alpha}}^{i}+2\left(\mathcal{D}^{\hat{\gamma} i} \sigma\right) M_{\hat{\gamma} \hat{\alpha}}-3\left(\mathcal{D}_{\hat{\alpha} k} \sigma\right) J^{k i}, \\
& \delta_{\sigma} \mathcal{D}_{\hat{a}}=\sigma \mathcal{D}_{\hat{a}}+\frac{\mathrm{i}}{2}\left(\Gamma_{\hat{a}}\right)^{\hat{\gamma} \hat{\delta}}\left(\mathcal{D}_{\hat{\gamma}}^{k} \sigma\right) \mathcal{D}_{\hat{\delta} k}-\left(\mathcal{D}^{\hat{b}} \sigma\right) M_{\hat{a} \hat{b}},
\end{aligned}
$$


while the super Weyl transformation of, for example, $\mathfrak{F}_{\hat{\alpha}}^{i \hat{\beta} j}$ is

$$
\delta_{\sigma} \mathfrak{F}_{\hat{\alpha}}^{i \hat{\beta} j}=\sigma \mathfrak{F}_{\hat{\alpha}}^{i \hat{\beta} j}-\frac{1}{2} \mathcal{D}_{\hat{\alpha}}^{i} \mathcal{D}^{\hat{\beta} j} \sigma+\frac{\mathrm{i}}{2} \varepsilon^{i j} \mathcal{D}_{\hat{\alpha}}^{\hat{\beta}} \sigma=\sigma \mathfrak{F}_{\hat{\alpha}}^{i \hat{\beta} j}-\frac{1}{4}\left[\mathcal{D}_{\hat{\alpha}}^{i}, \mathcal{D}^{\hat{\beta} j}\right] \sigma .
$$

Equation (3.27) implies

$$
\begin{aligned}
\delta_{\sigma} S^{i j} & =\sigma S^{i j}+\frac{\mathrm{i}}{4} \mathcal{D}^{\hat{\alpha}(i} \mathcal{D}_{\hat{\alpha}}^{j)} \sigma, \\
\delta_{\sigma} C_{\hat{a}}^{i j} & =\sigma C_{\hat{a}}^{i j}+\frac{\mathrm{i}}{2}\left(\Gamma_{\hat{a}}\right)^{\hat{\gamma} \hat{\delta}} \mathcal{D}_{\hat{\gamma}}^{(i} \mathcal{D}_{\hat{\delta}}^{j)} \sigma, \\
\delta_{\sigma} Y_{\hat{a} \hat{b}} & =\sigma Y_{\hat{a} \hat{b}}-\frac{\mathrm{i}}{4}\left(\Sigma_{\hat{a} \hat{b}}\right)^{\hat{\alpha} \hat{\beta}} \mathcal{D}_{\hat{\alpha}}^{k} \mathcal{D}_{\hat{\beta} k} \sigma .
\end{aligned}
$$

\section{The Weyl multiplet}

The 5D Weyl multiplet, constructed independently by two groups [19, 20] and [21, 22], consists of the following matter content: four fundamental one-forms - the vielbein $e_{\hat{m}}{ }^{a}$, the gravitini $\psi_{\hat{m} \hat{\alpha}}^{i}$, an $\mathrm{SU}(2)$ gauge field $\mathcal{V}_{\hat{m}}{ }^{i j}$, and a dilatation gauge field $b_{\hat{m}}$; and three covariant auxiliary fields - a real antisymmetric tensor $w_{\hat{a} \hat{b}}$, a fermion $\chi_{\hat{\alpha}}^{i}$, and a real auxiliary scalar $D$. In addition, there are three composite one-forms - the spin connection $\omega_{\hat{m}}^{\hat{a} \hat{b}}$, the $S$-supersymmetry connection $\phi_{\hat{m}_{\hat{\alpha}}}{ }^{i}$, and the special conformal connection $\mathfrak{f}_{\hat{m}}{ }^{\hat{a}}$ which are algebraically determined in terms of the other fields by imposing constraints on some of the curvature tensors.

In a standard component analysis, one begins by interpreting the seven one-forms appearing above as connections for the $5 \mathrm{D}$ superconformal algebra $\mathrm{F}^{2}(4)$. Associated with each connection is a two-form field strength, constructed in the usual manner from the superalgebra $\mathrm{F}^{2}(4)$. One wishes to algebraically constrain the spin, $S$-supersymmetry, and special conformal connections in terms of the other quantities: this can be accomplished by constraining respectively the vielbein curvature $R(P)_{\hat{m} \hat{n}}{ }^{\hat{a}}$, the gravitino curvature $R(Q)_{\hat{m} \hat{n} \hat{\alpha}}^{i}$, and the conformal Lorentz curvature $R(M)_{\hat{m} \hat{n}} \hat{a} \hat{b}$. However, the remaining one-forms cannot furnish an off-shell representation of a conformal supersymmetry algebra as the bosonic and fermionic degrees of freedom do not match, so one is led to introduce the additional covariant fields $w_{\hat{a} \hat{b}}$ (denoted $T_{\hat{a} \hat{b}}$ in $[21,22]$ and $v_{\hat{a} \hat{b}}$ in $[19,20]$ ), $\chi_{\hat{\alpha}}{ }^{i}$, and $D$. At this stage, one must determine how the presence of the auxiliary fields deforms the supersymmetry algebra, the curvatures, and the constraints imposed on the curvatures in a self-consistent way. In general, there is no unique solution, and indeed, the two original groups, as well as the recent work [52], each use different definitions for supersymmetry and for the curvatures.

In contrast, the technical advantage of a superspace approach is that once the supergeometry is completely specified and the Bianchi identities solved, one must only specify definitions for the component fields - their supersymmetry transformations and the corresponding curvatures are then completely determined. Our goal in this section is to demonstrate precisely how this occurs for the 5D Weyl multiplet. 


\subsection{Component fields and curvatures from superspace}

We begin by identifying the various component fields of the Weyl multiplet. Let us start with the vielbein and gravitino. These appear as the coefficients of $\mathrm{d} x^{\hat{m}}$ of the supervielbein $E^{\hat{A}}=\left(E^{\hat{a}}, E_{i}^{\hat{\alpha}}\right)=\mathrm{d} z^{\hat{M}} E_{\hat{M}} \hat{A}$. It is convenient to introduce the so-called double bar projection $[53,54]$, denoted by $E^{\hat{A}} \|$, that restricts to $\theta=\mathrm{d} \theta=0$, corresponding to the bosonic part $T^{*} \mathcal{M}^{5}$ of the cotangent bundle $T^{*} \mathcal{M}^{5 \mid 8}$, where $\mathcal{M}^{5}$ is the bosonic body of the curved superspace $\mathcal{M}^{5 \mid 8}$. Then we can define ${ }^{10}$

$$
e^{\hat{a}}=\mathrm{d} x^{\hat{m}} e_{\hat{m}}^{\hat{a}}:=E^{\hat{a}}\left\|, \quad \psi_{\hat{\alpha}}^{i}=\mathrm{d} x^{\hat{m}} \psi_{\hat{m} \hat{\alpha}}^{i}:=2 E_{\hat{\alpha}}^{i}\right\| .
$$

This is equivalent to defining $e_{\hat{m}}^{\hat{a}}=E_{\hat{m}}^{\hat{a}} \mid$ and $\psi_{\hat{m}_{\hat{\alpha}}^{i}}^{i}=2 E_{\hat{m}}{ }_{\hat{\alpha}} \mid$ where the single vertical bar denotes the usual component projection to $\theta=0$, i.e. $V(z)|:=V(z)|_{\theta=0}$ for any superfield $V(z)$. In like fashion the remaining fundamental and composite one-forms are found by taking the projections of the corresponding superforms,

$$
\mathcal{V}^{i j}:=\Phi^{i j}\|, \quad b:=B\|, \quad \omega^{\hat{a} \hat{b}}:=\Omega^{\hat{a} \hat{b}}\left\|, \quad \phi_{\hat{\alpha}}^{i}:=2 \mathfrak{F}_{\hat{\alpha}}^{i}\right\|, \quad f^{\hat{a}}:=\mathfrak{F}^{\hat{a}} \| .
$$

The additional auxiliary fields are contained within the curvature superfield $W_{\hat{\alpha} \hat{\beta}}$,

$$
w_{\hat{\alpha} \hat{\beta}}:=W_{\hat{\alpha} \hat{\beta}}\left|, \quad \chi_{\hat{\alpha}}^{i}:=\frac{3 \mathrm{i}}{32} X_{\hat{\alpha}}^{i}\right|, \quad D:=-\frac{3}{128} Y \mid .
$$

The normalizations we have chosen for $\chi_{\hat{\alpha}}^{i}$ and $D$ coincide with the normalizations of $[21,22]$ and [52]. The other independent components of the curvature superfield are given by $W_{\hat{a} \hat{b} \hat{\alpha}}{ }^{i} \mid$ and by $X_{\hat{a} \hat{b}}{ }^{i j} \mid$, and will turn out to be given by some of the component curvatures.

It should be mentioned that one can impose a Wess-Zumino gauge to fix the $\theta$ expansions of the super one-forms, so that they are completely determined by the above fields. This ensures that the entire physical content of the superspace geometry is accounted for. In practice, it is usually unnecessary to do this explicitly.

Now we may determine the so-called supercovariant curvatures. In terms of the connection one-forms, the covariant derivative $\nabla_{\hat{a}} \mid$ is defined by taking the double bar projection of equation (2.6), leading to

$$
e_{\hat{m}}^{\hat{a}} \nabla_{\hat{a}}\left|=\partial_{\hat{m}}-\frac{1}{2} \psi_{\hat{m}_{i}}^{\hat{\alpha}} \nabla_{\hat{\alpha}}^{i}\right|-\frac{1}{2} \omega_{\hat{m}}^{\hat{a} \hat{b}} M_{\hat{a} \hat{b}}-b_{\hat{m}} \mathbb{D}-\mathcal{V}_{\hat{m}}{ }^{i j} J_{i j}-\frac{1}{2} \phi_{\hat{m}}{ }^{\hat{\alpha} i} S_{\hat{\alpha} i}-\mathfrak{f}_{\hat{m}}{ }^{\hat{a}} K_{\hat{a}}
$$

where we have defined the lowest component of the superspace operator $\nabla_{\hat{\alpha}}^{i} \mid$ such that for an arbitrary tensor superfield $U$

$$
\left(\nabla_{\hat{\alpha}}^{i} \mid U\right)\left|=\left(\nabla_{\hat{\alpha}}^{i} U\right)\right|
$$

We interpret $\nabla_{\hat{\alpha}}^{i} \mid$ as the generator of supersymmetry. In what follows we will drop the bar projection from $\nabla_{\hat{a}} \mid$ when it is clear from context to which we are referring.

\footnotetext{
${ }^{10}$ We define the gravitino with a lowered spinor index and a raised $\mathrm{SU}(2)$ index. We follow similar conventions when defining other component fields.
} 
It will be convenient to also introduce the spin, dilatation, and $\mathrm{SU}(2)$ covariant derivative

$$
\begin{aligned}
& \mathcal{D}_{\hat{m}}:=\partial_{\hat{m}}-\frac{1}{2} \omega_{\hat{m}}^{\hat{b} \hat{c}} M_{\hat{b} \hat{c}}-b_{\hat{m}} \mathbb{D}-\mathcal{V}_{\hat{m}}{ }^{i j} J_{i j}, \\
& \mathcal{D}_{\hat{a}}:=e_{\hat{a}}{ }^{\hat{m}} \mathcal{D}_{\hat{m}}=e_{\hat{a}}{ }^{\hat{m}} \partial_{\hat{m}}-\frac{1}{2} \omega_{\hat{a}}^{\hat{b} \hat{c}} M_{\hat{b} \hat{c}}-b_{\hat{a}} \mathbb{D}-\mathcal{V}_{\hat{a}}{ }^{i j} J_{i j},
\end{aligned}
$$

where

$$
\omega_{\hat{a}}^{\hat{b} \hat{c}}:=e_{\hat{a}}^{\hat{m}} \omega_{\hat{m}}^{\hat{b} \hat{c}}, \quad b_{\hat{a}}:=e_{\hat{a}}^{\hat{m}} b_{\hat{m}}, \quad \mathcal{V}_{\hat{a}}^{i j}:=e_{\hat{a}}^{\hat{m}} \mathcal{V}_{\hat{m}}^{i j}
$$

The supercovariant curvature tensors are given by

$$
\begin{aligned}
{\left[\nabla_{\hat{a}}, \nabla_{\hat{b}}\right]=} & -R(P)_{\hat{a} \hat{b}}^{\hat{b}} \nabla_{\hat{c}}-R(Q)_{\hat{a} \hat{b}{ }^{\hat{\alpha}}} \nabla_{\hat{\alpha}}^{i} \mid-\frac{1}{2} R(M)_{\hat{a} \hat{b}} \hat{\hat{b}} \hat{d} M_{\hat{c} \hat{d}}-R(J)_{\hat{a} \hat{b}}^{i j} J_{i j} \\
& -R(\mathbb{D})_{\hat{a} \hat{b} b} \mathbb{D}-R(S)_{\hat{a} \hat{b}}^{\hat{\hat{b}} k} S_{\hat{\gamma} k}-R(K)_{\hat{a} \hat{b}}^{\hat{c}} K_{\hat{c}}
\end{aligned}
$$

and are found by taking the component projections of the curvature tensors in $(2.35 \mathrm{c})$. We have introduced the expressions

$$
R(P)_{\hat{a} \hat{b}}^{\hat{c}}=\mathscr{T}_{\hat{a} \hat{b}}^{\hat{c}}\left|, \quad R(Q)_{\hat{a} \hat{b} \hat{\alpha}}^{i}=\mathscr{T}_{\hat{a} \hat{b} \hat{\alpha}}^{i}\right|
$$

for the lowest components of the superspace torsion tensors to match the usual component nomenclature.

At this stage there are two distinct expressions we can give for each of the curvature tensors. Let us demonstrate with $R(P)_{\hat{a} \hat{b}} \hat{b}$. We can write two equivalent expressions for the double-bar projection of the torsion two-form $\mathscr{T}^{\hat{c}}$,

$$
\mathscr{T}^{\hat{c}} \|=\frac{1}{2} \mathrm{~d} x^{\hat{n}} \wedge \mathrm{d} x^{\hat{m}} \mathscr{T}_{\hat{m} \hat{n}}^{\hat{c}} \mid=\mathrm{d} x^{\hat{n}} \wedge \mathrm{d} x^{\hat{m}} \mathcal{D}_{[\hat{m}} e_{\hat{n}]}^{\hat{c}}
$$

and

$$
\begin{aligned}
\mathscr{T}^{\hat{c}} \| & =\frac{1}{2}(-1)^{\varepsilon_{\hat{A}} \varepsilon_{\hat{B}}} E^{\hat{A}} \wedge E^{\hat{B}} \mathscr{T}_{\hat{A} \hat{B}}^{\hat{c}} \| \\
& =\frac{1}{2} \mathrm{~d} x^{\hat{n}} \wedge \mathrm{d} x^{\hat{m}}\left(e_{\hat{m}}^{\hat{a}} e_{\hat{n}}^{\hat{b}} \mathscr{T}_{\hat{a} \hat{b}}^{\hat{c}}\left|+e_{[\hat{m}}^{\hat{a}} \psi_{\hat{n}] j}^{\hat{\beta}} \mathscr{T}_{\hat{a}}^{j \hat{\beta}}\right|-\frac{1}{4} \psi_{\hat{m} i}^{\hat{\alpha}} \psi_{\hat{n} j}^{\hat{\beta}} \mathscr{T}_{\hat{\alpha} \hat{\beta}}^{i j} \hat{c} \mid\right) \\
& =\frac{1}{2} \mathrm{~d} x^{\hat{n}} \wedge \mathrm{d} x^{\hat{m}}\left(e_{\hat{m}}{ }^{\hat{a}} e_{\hat{n}}^{\hat{b}} R(P)_{\hat{a} \hat{b}}{ }^{\hat{c}}+\frac{\mathrm{i}}{2} \psi_{\hat{m} j} \Gamma^{\hat{c}} \psi_{\hat{n}}{ }^{j}\right) .
\end{aligned}
$$

Equating the two expressions provides a definition for the supercovariant curvature $R(P)_{\hat{a} \hat{b}} \hat{c}$. Proceeding in this way for the other curvature two-forms, we find the following definitions:

$$
\begin{aligned}
R(P)_{\hat{a} \hat{b}}^{\hat{c}}:= & 2 e_{\hat{a}}^{\hat{m}} e_{\hat{b}}^{\hat{n}} \mathcal{D}_{[\hat{m}} e_{\hat{n}]}^{\hat{c}}-\frac{\mathrm{i}}{2} \psi_{\hat{a} j} \Gamma^{\hat{c}} \psi_{\hat{b}}^{j} \\
R(Q)_{\hat{a} \hat{b} \hat{\alpha}}^{i}:= & e_{\hat{a}}^{\hat{m}} e_{\hat{b}}^{\hat{n}} \mathcal{D}_{[\hat{m}} \psi_{\hat{n}]} \hat{\hat{\alpha}}^{i}+\mathrm{i}\left(\Gamma_{[\hat{a}} \phi_{\hat{b}]}{ }^{i}\right)_{\hat{\alpha}}+\frac{1}{2} w_{\hat{c} \hat{d}}\left(\Sigma^{\hat{c} \hat{d}} \Gamma_{[\hat{a}} \psi_{\hat{b}]}{ }^{i}\right)_{\hat{\alpha}}, \\
R(M)_{\hat{a} \hat{b}}^{\hat{c} \hat{d}}:= & \mathcal{R}(\omega)_{\hat{a} \hat{b}}^{\hat{c} \hat{d}}+8 \delta_{[\hat{a}}\left[c_{\hat{T}_{\hat{b}]}}{ }^{d]}-2 \psi_{[\hat{a} j} \Sigma^{\hat{c} \hat{d}} \phi_{\hat{b}]}^{j}-2 \mathrm{i}\left(\psi_{[\hat{a} j} \Gamma_{\hat{b}]} R(Q)^{\hat{c} \hat{d} j}\right)\right. \\
& -\frac{32 \mathrm{i}}{3}\left(\psi_{[\hat{a} j} \Gamma_{\hat{b}]} \Sigma^{\hat{c} \hat{d}} \chi^{j}\right)+\frac{\mathrm{i}}{2} \psi_{\hat{a} j} \psi_{\hat{b}}^{j} w^{\hat{c} \hat{d}},
\end{aligned}
$$




$$
\begin{aligned}
R(J)_{\hat{a} \hat{b}}^{i j} & :=\mathcal{R}(\mathcal{V})_{\hat{a} \hat{b}}^{i j}-3\left(\psi_{[\hat{a}}^{(i} \phi_{\hat{b}]}^{j)}\right)-16 \mathrm{i}\left(\psi_{[\hat{a}}^{(i} \Gamma_{\hat{b}]} \chi^{j)}\right), \\
R(\mathbb{D})_{\hat{a} \hat{b}} & :=2 e_{\hat{a}}^{\hat{m}} e_{\hat{b}}^{\hat{n}} \partial_{[\hat{m}} b_{\hat{n}]}+4 \mathfrak{f}_{[\hat{a} \hat{b}]}+\left(\psi_{[\hat{a} j} \phi_{\hat{b}]}^{j}\right)+\frac{16 \mathrm{i}}{3}\left(\psi_{[\hat{a} k} \Gamma_{\hat{b}]} \chi^{k}\right),
\end{aligned}
$$

where we have introduced

$$
\psi_{\hat{a} j}^{\hat{\beta}}:=e_{\hat{a}}^{\hat{m}} \psi_{\hat{m}_{j}}^{\hat{\beta}}, \quad \phi_{\hat{a} j}^{\hat{\beta}}:=e_{\hat{a}}^{\hat{m}} \phi_{\hat{m}_{j}}^{\hat{\beta}}, \quad \mathfrak{f}_{\hat{a}}^{\hat{b}}=e_{\hat{a}} \hat{m}_{\mathfrak{f}_{\hat{m}}}^{\hat{b}}
$$

and the curvatures

$$
\begin{aligned}
& \mathcal{R}(\omega)_{\hat{a} \hat{b}}^{\hat{b} \hat{d}}:=2 e_{\hat{a}}^{\hat{m}} e_{\hat{b}}^{\hat{n}}\left(\partial_{[\hat{m}} \omega_{\hat{n}]}{ }^{\hat{d} \hat{d}}-2 \omega_{[\hat{m}}{ }^{\hat{c} \hat{e}} \omega_{\left.\hat{n}] e^{\hat{e}}\right)}^{\hat{d}}\right), \\
& \mathcal{R}(\mathcal{V})_{\hat{a} \hat{b}}{ }^{i j}:=2 e_{\hat{a}}^{\hat{m}} e_{\hat{b}}^{\hat{n}}\left(\partial_{[\hat{m}} \mathcal{V}_{\hat{n}]}^{i j}+\mathcal{V}_{[\hat{m}}^{k(i} \mathcal{V}_{\hat{n}]}^{j)} k\right) .
\end{aligned}
$$

The supercovariant forms of $R(S)_{\hat{a} \hat{b} \hat{\alpha}}^{i}$ and $R(K)_{\hat{a} \hat{b}}^{\hat{c}}$ are a good deal more complicated, so we do not give them here.

\subsection{Analysis of the curvature constraints}

We have not yet employed the constraints imposed by superspace on the curvatures. They are

$$
\begin{aligned}
& R(P)_{\hat{a} \hat{b}}^{\hat{c}}=-\tilde{w}_{\hat{a} \hat{b}}^{\hat{c}} \equiv-\frac{1}{2} \varepsilon_{\hat{a} \hat{b}}^{\hat{c} \hat{b} \hat{e}} w_{\hat{d} \hat{e}}, \\
& \left(\Gamma^{\hat{a}} R(Q)_{\hat{a} \hat{b}}{ }^{i}\right)_{\hat{\alpha}}=-\frac{32}{3}\left(\Gamma_{\hat{b}} \chi^{i}\right)_{\hat{\alpha}}, \\
& R(M)_{\hat{a} \hat{b}}^{\hat{c} \hat{b}}=-\frac{32}{3} \delta_{\hat{a}}^{\hat{c}} D-w_{\hat{a} \hat{d}} w^{\hat{c} \hat{d}}-\nabla^{\hat{d}} \tilde{w}_{\hat{d} \hat{a}}{ }^{\hat{c}},
\end{aligned}
$$

and respectively determine the spin connection, the $S$-supersymmetry connection, and the $K$-connection. In contrast to previous conventions employed in the literature, these are actually $S$-invariant constraints. The reason for this is that the superspace operators $\nabla_{\hat{\alpha}}^{i}$ and $\nabla_{\hat{a}}$ have the same algebra with $S_{\hat{\alpha} i}$ as one finds in the superconformal algebra $\mathrm{F}^{2}(4)$. The price one pays for this simplicity is that the composite connections will turn out to depend rather more significantly on the auxiliary fields $w_{\hat{a} \hat{b}}, \chi_{\hat{\alpha}}^{i}$ and $D$ than one might have wished.

The first constraint (4.16a) determines the spin connection to be

$$
\omega_{\hat{a} \hat{b} \hat{c}}=\omega(e)_{\hat{a} \hat{b} \hat{c}}+\frac{i}{4}\left(\psi_{\hat{a} k} \Gamma_{\hat{c}} \psi_{\hat{b}}^{k}+\psi_{\hat{c} k} \Gamma_{\hat{b}} \psi_{\hat{a}}^{k}-\psi_{\hat{b} k} \Gamma_{\hat{a}} \psi_{\hat{c}}^{k}\right)+2 b_{[\hat{b}} \eta_{\hat{c}] \hat{a}}-\frac{1}{4} \varepsilon_{\hat{a} \hat{b} \hat{c}} \hat{d} \hat{e} w_{\hat{d} \hat{e}},
$$

where $\omega(e)_{\hat{a} \hat{b} \hat{c}}=-\frac{1}{2}\left(\mathcal{C}_{\hat{a} \hat{b} \hat{c}}+\mathcal{C}_{\hat{c} \hat{a} \hat{b}}-\mathcal{C}_{\hat{b} \hat{c} \hat{a}}\right)$ is the usual spin connection of general relativity, given in terms of the anholonomy coefficient $\mathcal{C}_{\hat{m} \hat{n}}{ }^{\hat{a}}:=2 \partial_{[\hat{m}} e_{\hat{n}]}{ }^{\hat{a}}$. Note that the spin connection $\omega_{\hat{a} \hat{b} \hat{c}}$ possesses torsion: in addition to the usual contribution from the gravitino bilinears, there is additional bosonic torsion from the auxiliary field $w_{\hat{a} \hat{b}}$.

From the second constraint (4.16b), we find the $S$-supersymmetry connection

$$
\mathrm{i} \phi_{\hat{m}}^{i}=\frac{8}{3} \Gamma_{\hat{m}} \chi^{i}+\frac{1}{3}\left(\Gamma^{[\hat{p}} \delta_{\hat{m}}^{\hat{q}]}+\frac{1}{4} \Gamma_{\hat{m}} \Sigma^{\hat{p} \hat{q}}\right)\left(\Psi_{\hat{p} \hat{q}}^{i}+w_{\hat{a} \hat{b}} \sum^{\hat{a} \hat{b}} \Gamma_{[\hat{p}} \psi_{\hat{q}]}^{i}\right),
$$


where we have suppressed spinor indices for legibility and introduced the gravitino field strength $\Psi_{\hat{m} \hat{n} \hat{\alpha}}^{i}:=2 \mathcal{D}_{[\hat{m}} \psi_{\hat{n}]}^{i}$. Reinserting this back into the original expression for $R(Q)$, we find that

$$
R(Q)_{\hat{a} \hat{b}}^{i}=\frac{1}{2} \Pi_{\hat{a} \hat{b}} \hat{d} \hat{d}\left(\Psi_{\hat{c} \hat{d}}^{i}-w_{\hat{c} \hat{d}} \Gamma^{e} \psi_{e}^{i}-\tilde{w}_{\hat{a} \hat{b}}{ }^{\hat{c}} \psi_{c}^{i}\right)-\frac{16}{3} \Sigma_{\hat{a} \hat{b}} \chi^{i}
$$

where the spinor projection operator

$$
\Pi_{\hat{a} \hat{b}}^{\hat{c} \hat{d}}:=\delta_{\hat{a}}^{[\hat{c}} \delta_{\hat{b}}^{\hat{d}]}+\frac{2}{3} \delta_{[\hat{a}}^{[\hat{c}} \Gamma_{\hat{b}]} \Gamma^{\hat{d}]}-\frac{1}{3} \Sigma_{\hat{a} \hat{b}} \Sigma^{\hat{c} \hat{d}}, \quad \Gamma^{\hat{a}} \Pi_{\hat{a} \hat{b}}^{\hat{c} \hat{d}}=0, \quad \Pi^{2}=\Pi
$$

projects onto the $\Gamma$-traceless part of a spinor-valued two-form. It is convenient to introduce a separate symbol $\hat{R}(Q)_{\hat{a} \hat{b} \hat{\alpha}}^{i}$ for the first term of (4.19),

$$
\hat{R}(Q)_{\hat{a} \hat{b}}^{i}=\frac{1}{2} \Pi_{\hat{a} \hat{b}} \hat{c} \hat{d}\left(\Psi_{\hat{c} \hat{d}}^{i}-w_{\hat{c} \hat{d}} \Gamma^{\hat{e}} \psi_{\hat{e}}^{i}-\tilde{w}_{\hat{a} \hat{b}}^{\hat{c}} \psi_{\hat{c}}^{i}\right),
$$

corresponding to its $\Gamma$-traceless part. Using (2.36b), we find that one of the remaining components of the superspace curvature is determined,

$$
W_{\hat{\alpha} \hat{\beta} \hat{\gamma}}^{i} \mid=\mathrm{i}\left(\Sigma^{\hat{a} \hat{b}}\right)_{\hat{\alpha} \hat{\beta}} \hat{R}(Q)_{\hat{a} \hat{b} \hat{\gamma}}^{i}=\mathrm{i} \Psi_{(\hat{\gamma} \hat{\beta} \hat{\alpha})}^{i}+\mathrm{i} w_{(\hat{\gamma} \hat{\beta}} \psi_{\hat{\alpha}) \hat{\delta}} \hat{\delta}^{\hat{i}}-\mathrm{i} w_{\hat{\delta}(\hat{\alpha}} \psi_{\hat{\gamma} \hat{\beta})^{\hat{\delta}}}^{i}
$$

From the third constraint (4.16c), one can show that

$$
\begin{aligned}
\mathfrak{f}_{\hat{a}}^{\hat{b}}=- & \frac{2}{3} \delta_{\hat{b}}^{\hat{a}} D-\frac{1}{6} w_{\hat{a} \hat{c}} w^{\hat{b} \hat{c}}+\frac{1}{48} \delta_{\hat{a}}^{\hat{b}} w^{\hat{c} \hat{d}} w_{\hat{c} \hat{d}}-\frac{1}{6} \nabla^{\hat{c}} \tilde{w}_{\hat{c} \hat{a}}^{\hat{b}}-\frac{1}{6} \mathcal{R}_{\hat{a}} \hat{b}^{\hat{b}}(\omega)+\frac{1}{48} \delta_{\hat{a}}^{\hat{b}} \mathcal{R}(\omega) \\
& -\frac{\mathrm{i}}{6}\left(\psi_{\hat{d}} \Gamma_{\hat{a}} \hat{R}(Q)^{\hat{b} \hat{d} j}\right)+\frac{1}{3}\left(\psi_{[\hat{a} j} \Sigma^{\hat{b} \hat{c}} \phi_{\hat{c}]^{j}}\right)-\frac{1}{24} \delta_{\hat{a}}^{\hat{b}}\left(\psi_{\hat{c} j} \Sigma^{\hat{c} \hat{d}} \phi_{\hat{d}}^{j}\right) \\
& -\frac{\mathrm{i}}{12}\left(\psi_{\hat{a} j} \psi_{\hat{c}}^{j}\right) w^{\hat{b} \hat{c}}+\frac{\mathrm{i}}{96} \delta_{\hat{a}}^{\hat{b}}\left(\psi_{\hat{c} j} \psi_{\hat{d}}^{j}\right) w^{\hat{c} \hat{d}}
\end{aligned}
$$

where $\mathcal{R}_{\hat{a}}^{\hat{b}}(\omega)=\mathcal{R}_{\hat{a} \hat{c}} \hat{b} \hat{c}(\omega)$ and $\mathcal{R}(\omega)=\mathcal{R}_{\hat{a}}{ }^{\hat{a}}(\omega)$. In principle, one can reinsert this expression into $R(M)_{\hat{a} \hat{b}} \hat{c} \hat{d}$. The result is quite complicated; we remark only that it can be written

$$
\begin{aligned}
R(M)_{\hat{a} \hat{b}} \hat{c}^{\hat{d}}= & C(\omega)_{\hat{a} \hat{b}}^{\hat{c} \hat{d}}-\frac{4}{3} \delta_{[\hat{a}}^{[\hat{c}} w_{\hat{b}] \hat{e}} w^{\hat{d}] \hat{e}}-\frac{4}{3} \nabla^{\hat{e}} \tilde{w}_{\hat{e}[\hat{a}}^{[\hat{c}} \delta_{\hat{b}]}^{\hat{d}]} \\
& +\delta_{[\hat{a}}^{[\hat{c}} \delta_{\hat{b}]}^{\hat{d}]}\left(\frac{1}{6} w^{\hat{e} \hat{f}} w_{\hat{e} \hat{f}}-\frac{16}{3} D\right)+(\text { explicit gravitino terms }),
\end{aligned}
$$

where $C(\omega)_{\hat{a} \hat{b}} \hat{c}^{\hat{d}}=\mathcal{R}(\omega)_{\hat{a} \hat{b}} \hat{c}^{\hat{d}}-\frac{4}{3} \delta_{[\hat{a}}^{[\hat{c}} \mathcal{R}(\omega)_{\hat{b}]}^{\hat{d}]}+\frac{1}{6} \delta_{[\hat{a}}^{[\hat{c}} \delta_{\hat{b}]}^{\hat{d}]} \mathcal{R}(\omega)$ is the traceless part of the tensor $\mathcal{R}(\omega)_{\hat{a} \hat{b}} \hat{c} \hat{d}$. This is not quite the usual Weyl tensor because of the presence of bosonic torsion in the spin connection. The superspace expression for $\mathscr{R}(M)_{\hat{a} \hat{b}} \hat{b} \hat{d}$ in principle determines $W_{\hat{\alpha} \hat{\beta} \hat{\gamma} \hat{\delta}} \mid$; however, we will find a more useful form of this expression using a different method shortly.

For the remaining dimension-2 curvatures, we find

$$
R(\mathbb{D})_{\hat{a} \hat{b}}=-\nabla^{\hat{c}} \tilde{w}_{\hat{c} \hat{a} \hat{b}}, \quad R(J)_{\hat{a} \hat{b}}^{i j}=-\frac{3 \mathrm{i}}{4} X_{\hat{a} \hat{b}}{ }^{i j} \mid .
$$

The first equation is automatically satisfied upon substituting into $R(\mathbb{D})$ the expression for $\mathfrak{f}_{\hat{m}}{ }^{\hat{a}}$. The second equation serves as a definition for the remaining undetermined component $X_{\hat{a} \hat{b}}{ }^{i j} \mid$ of the Weyl superfield. 


\subsection{Supersymmetry transformations of the fundamental fields}

Here we present the complete $Q, S$, and $K$ transformations for the fundamental fields of the Weyl multiplet. The transformations of the one-forms follow from eq. (2.21), while those of the covariant fields can be read off from (2.33):

$$
\begin{aligned}
& \delta e_{\hat{m}}^{\hat{a}}=\mathrm{i}\left(\xi_{j} \Gamma^{\hat{a}} \psi_{\hat{m}^{j}}\right), \\
& \delta \psi_{\hat{m} \hat{\alpha}}^{i}=2 \mathcal{D}_{\hat{m}} \xi_{\hat{\alpha}}^{i}+w_{\hat{c} \hat{d}}\left(\Sigma^{\hat{c} \hat{d}} \Gamma_{\hat{m}} \xi^{i}\right)_{\hat{\alpha}}+2 \mathrm{i}\left(\Gamma_{\hat{m}} \eta^{i}\right)_{\hat{\alpha}}, \\
& \delta \mathcal{V}_{\hat{m}}{ }^{i j}=3 \xi^{(i} \phi_{\hat{m}}{ }^{j)}+16 \mathrm{i} \xi^{(i} \Gamma_{\hat{m}} \chi^{j)}-3 \eta^{(i} \psi_{\hat{m}}{ }^{j)} \text {, } \\
& \delta b_{\hat{m}}=-\xi_{k} \phi_{\hat{m}}{ }^{k}-\frac{16 \mathrm{i}}{3} \xi_{k} \Gamma_{\hat{m}} \chi^{k}-\eta_{k} \psi_{\hat{m}}{ }^{k}-2 e_{\hat{m}}{ }^{\hat{a}} \Lambda_{K \hat{a}}, \\
& \delta w_{\hat{a} \hat{b}}=2 \mathrm{i} \xi_{i} R(Q)_{\hat{a} \hat{b}}{ }^{i}, \\
& \delta \chi_{\hat{\alpha}}^{i}=\frac{1}{2} \xi_{\hat{\alpha}}^{i} D+\frac{3}{128}\left(\nabla_{\hat{a}} w_{\hat{b} \hat{c}}\right)\left(3\left(\Sigma^{\hat{b} \hat{c}} \Gamma^{\hat{a}} \xi^{i}\right)_{\hat{\alpha}}+\left(\Gamma^{\hat{a}} \Sigma^{\hat{b} \hat{c}} \xi^{i}\right)_{\hat{\alpha}}\right) \\
& -\frac{1}{16} R(J)_{\hat{a} \hat{b}{ }^{i}}\left(\Sigma^{\hat{a} \hat{b}} \xi^{j}\right)_{\hat{\alpha}}-\frac{3 \mathrm{i}}{16} w_{\hat{a} \hat{b}}\left(\Sigma^{\hat{a} \hat{b}} \eta^{i}\right)_{\hat{\alpha}}, \\
& \delta D=2 \mathrm{i}\left(\xi_{j} \not \nabla \chi^{j}\right)+2\left(\eta_{j} \chi^{j}\right) .
\end{aligned}
$$

One can also derive the transformations of the composite one-forms from (2.21). For example, the transformations for the spin connection and the $S$-supersymmetry connection are

$$
\begin{aligned}
\delta \omega_{\hat{m}}^{\hat{a} \hat{b}}= & -\mathrm{i} \xi_{j} \psi_{\hat{m}}^{j} w^{\hat{a} \hat{b}}+2 \mathrm{i} \xi_{j} \Gamma_{\hat{m}} R(Q)^{\hat{a} \hat{b} j}+\frac{32 \mathrm{i}}{3} \xi_{j} \Gamma_{\hat{m}} \Sigma^{\hat{a} \hat{b}} \chi^{j}+2 \xi_{k} \Sigma^{\hat{a} \hat{b}} \phi_{\hat{m}}^{k} \\
& -2 \eta_{j} \Sigma^{\hat{a} \hat{b}} \psi_{\hat{m}^{j}}{ }^{j}+4 e_{\hat{m}}{ }^{[\hat{a}} \Lambda_{K}^{\hat{b}]}, \\
\delta \phi_{\hat{m} \hat{\alpha}}^{i}= & -2 \mathrm{i} \mathfrak{f}_{\hat{m}}^{\hat{a}}\left(\Gamma_{\hat{a}} \xi^{i}\right)_{\hat{\alpha}}-\frac{16}{3}\left(\xi_{j} \psi_{\hat{m}}{ }^{j}\right) \chi_{\hat{\alpha}}^{i}-\frac{8 \mathrm{i}}{3}\left(\Gamma_{\hat{m}} \xi^{i}\right)_{\hat{\alpha}} D \\
& -\frac{\mathrm{i}}{8}\left(\nabla_{\hat{a}} w_{\hat{b} \hat{c}}\right)\left(\Sigma^{\hat{b} \hat{c}} \Gamma^{\hat{a}} \Gamma_{\hat{m}}+3 \Gamma^{\hat{a}} \Sigma^{\hat{b} \hat{c}} \Gamma_{\hat{m}}\right)_{\hat{\alpha}}{ }^{\hat{\beta}} \xi_{\hat{\beta}}^{i}+\frac{\mathrm{i}}{3} R(J)_{\hat{a} \hat{b}}{ }^{i j}\left(\Sigma^{\hat{a} \hat{b}} \Gamma_{\hat{m}} \xi_{j}\right)_{\hat{\alpha}} \\
& +2 \mathcal{D}_{\hat{m}} \eta_{\hat{\alpha}}^{i}+\mathrm{i} \Lambda_{K}^{\hat{b}}\left(\Gamma_{\hat{b}} \psi_{\hat{m}}{ }^{i}\right)_{\hat{\alpha}},
\end{aligned}
$$

where $\Lambda_{K}^{\hat{a}}$ parametrizes the special conformal transformations. We do not give here the transformation rule for $\mathfrak{f}_{\hat{m}}{ }^{\hat{a}}$ as it is quite complicated.

\subsection{A new choice for component constraints}

As already alluded to, the component constraints (4.16) we have found from superspace are quite interesting from a technical standpoint: they are $S$-invariant. This is reflected in the fact that the $S$-supersymmetry transformations of the various one-forms are exactly those derived from the algebra $F^{2}(4)$. However, this comes with a price: we must introduce bosonic torsion involving the field $w_{\hat{a} \hat{b}}$ into the spin connection. Similarly, the $S$-supersymmetry and special conformal connections (4.18) and (4.23) include additional contributions from the auxiliary fields. The last case is particularly inconvenient - it reflects the fact that $R(M)_{\hat{a} \hat{b} \hat{c} \hat{d}}$ is not just a minimally covariantized version of the Weyl tensor, but depends additionally on the auxiliary fields $D, \chi_{\hat{\alpha}}{ }^{i}$, and $w_{\hat{a} \hat{b}}$. From a component point of view, it would be more convenient to extract these dependences so that the 
component fields and curvatures are as simply defined as possible. This will turn out to lead to a formulation that more closely resembles those of [19-23].

Let us begin by introducing new definitions for the composite spin, $S$-supersymmetry, and $K$-connections:

$$
\begin{aligned}
\hat{\omega}_{\hat{a} \hat{b} \hat{c}} & :=\omega_{\hat{a} \hat{b} \hat{c}}+\frac{1}{2} \tilde{w}_{\hat{a} \hat{b} \hat{c}}, \\
\mathrm{i} \hat{\phi}_{\hat{m}^{i}}{ }^{i} & :=\mathrm{i} \phi_{\hat{m}^{i}}{ }^{i}-\frac{8}{3} \Gamma_{\hat{m}} \chi^{i}, \\
\hat{\mathfrak{f}}_{\hat{a}}^{\hat{b}} & :=\mathfrak{f}_{\hat{a}}^{\hat{b}}+\frac{2}{3} \delta_{\hat{a}}^{\hat{b}} D+\frac{1}{4} w_{\hat{a} \hat{d}} w^{\hat{b} \hat{d}}+\frac{1}{4} \nabla^{\hat{c}} \tilde{w}_{\hat{c} \hat{a}}^{\hat{b}}-\frac{3}{64} w^{\hat{c} \hat{d}} w_{\hat{c} \hat{d}} \delta_{\hat{a}}^{\hat{b}} .
\end{aligned}
$$

These definitions actually correspond to a redefinition of the superspace vector covariant derivative,

$$
\begin{aligned}
\hat{\nabla}_{\hat{a}}= & \nabla_{\hat{a}}-\frac{1}{4} \tilde{W}_{\hat{a} \hat{b} \hat{c}} M^{\hat{b} \hat{c}}+\frac{1}{8} X^{\hat{\beta} i}\left(\Gamma_{\hat{a}}\right)_{\hat{\beta}}^{\hat{\alpha}} S_{\hat{\alpha} i}+\frac{1}{64}\left(Y+3 W^{\hat{b} \hat{c}} W_{\hat{b} \hat{c}}\right) K_{\hat{a}} \\
& -\frac{1}{4}\left(\nabla^{\hat{c}} \tilde{W}_{\hat{c} \hat{a}}^{\hat{b}}\right) K_{\hat{b}}-\frac{1}{4} W_{\hat{a} \hat{d}} W^{\hat{b} \hat{d}} K_{\hat{b}} .
\end{aligned}
$$

We discuss further this superspace interpretation in appendix C.

The new curvatures given by the algebra $\left[\hat{\nabla}_{\hat{a}}, \hat{\nabla}_{\hat{b}}\right]$ are

$$
\begin{aligned}
& \hat{R}(P)_{\hat{a} \hat{b}}^{\hat{c}}=2 e_{\hat{a}}^{\hat{m}} e_{\hat{b}}^{\hat{n}} \hat{\mathcal{D}}_{[\hat{m}} e_{\hat{n}]}^{\hat{c}}-\frac{\mathrm{i}}{2} \psi_{\hat{a} j} \Gamma^{\hat{c}} \psi_{\hat{b}}^{j}, \\
& \hat{R}(Q)_{\hat{a} \hat{b} \hat{\alpha}}^{i}=e_{\hat{a}}^{\hat{m}} e_{\hat{b}}^{\hat{n}} \hat{\mathcal{D}}_{[\hat{m}} \psi_{\hat{n}] \hat{\alpha}}^{i}+\mathrm{i}\left(\Gamma_{[\hat{a}} \hat{\phi}_{\hat{b}]}^{i}\right)_{\hat{\alpha}} \\
& +\frac{1}{8} w_{\hat{c} \hat{d}}\left(3\left(\Sigma^{\hat{c} \hat{d}} \Gamma_{[\hat{a}}\right)_{\hat{\alpha}}^{\hat{\beta}}-\left(\Gamma_{[\hat{a}} \Sigma^{\hat{c} \hat{d}}\right)_{\hat{\alpha}}^{\hat{\beta}}\right) \psi_{\hat{b}] \hat{\beta}}^{i},
\end{aligned}
$$

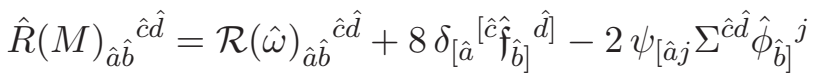

$$
\begin{aligned}
& +\frac{16 \mathrm{i}}{3} \delta_{[\hat{a}}^{[\hat{c}} \psi_{\hat{b}] i} \Gamma^{\hat{d}]} \chi^{i}-\mathrm{i} \psi_{[\hat{a} i}\left(\Gamma_{\hat{b}]} \hat{R}(Q)^{\hat{c} \hat{d} i}+2 \Gamma^{[\hat{c}} \hat{R}(Q)_{\hat{b}]}^{\hat{d}] i}\right) \\
& +\frac{\mathrm{i}}{2} \psi_{\hat{a} j} \psi_{\hat{b}}{ }^{j} w^{\hat{c} \hat{d}}-\frac{\mathrm{i}}{4}\left(\psi_{\hat{a} j} \Gamma_{\hat{e}} \psi_{b}{ }^{j}\right) \tilde{w}^{\hat{c} \hat{d} \hat{e}}, \\
& \hat{R}(J)_{\hat{a} \hat{b}}^{i j}=\mathcal{R}(\mathcal{V})_{\hat{a} \hat{b}}^{i j}-3 \psi_{[\hat{a}}^{\left(i, \hat{\phi}_{\hat{b}]}^{j)}\right.}-8 \mathrm{i} \psi_{[\hat{a}}^{(i)} \Gamma_{\hat{b}]} \chi^{j)}, \\
& \hat{R}(\mathbb{D})_{\hat{a} \hat{b}}=2 e_{\hat{a}}^{\hat{m}} e_{\hat{b}}^{\hat{n}} \partial_{[\hat{m}} b_{\hat{n}]}+4 \hat{\mathfrak{f}}_{[\hat{a} \hat{b}]}+\psi_{[\hat{a} j} \hat{\phi}_{\hat{b}]}^{j}+\frac{8 \mathrm{i}}{3} \psi_{[\hat{a} j} \Gamma_{\hat{b}]} \chi^{j},
\end{aligned}
$$

where we have introduced

$$
\hat{\mathcal{D}}_{\hat{a}}=e_{\hat{a}}^{\hat{m}} \partial_{\hat{m}}-\frac{1}{2} \hat{\omega}_{\hat{a}}^{\hat{b} \hat{c}} M_{\hat{b} \hat{c}}-b_{\hat{a}} \mathbb{D}-\mathcal{V}_{\hat{a}}^{i j} J_{i j}, \quad \mathcal{D}_{\hat{m}}=e_{\hat{m}}{ }^{\hat{a}} \hat{\mathcal{D}}_{\hat{a}} .
$$

We postpone for the moment a discussion of $\hat{R}(S)_{\hat{a} \hat{b}} \hat{\alpha} i$ and $\hat{R}(K)_{\hat{a} \hat{b}}{ }^{\hat{c}}$.

The curvatures turn out to obey the constraints

$$
\hat{R}(P)_{\hat{a} \hat{b}} \hat{c}^{\hat{b}}=0, \quad\left(\Gamma^{\hat{a}}\right)_{\hat{\alpha}}^{\hat{\beta}} \hat{R}(Q)_{\hat{a} \hat{b} \hat{\beta}}{ }^{i}=0, \quad \hat{R}(M)_{\hat{a} \hat{b}} \hat{\hat{b}} \hat{b}=0 .
$$

These coincide with the constraints usually imposed in the component formulations and are not $S$-invariant. This is a consequence of the redefinition of the auxiliary connections, 
which deforms their $S$-supersymmetry transformations. Equivalently, $\left[S_{\hat{\alpha} i}, \hat{\nabla}_{\hat{a}}\right]$ is no longer given in superspace simply by $\mathrm{i}\left(\Gamma_{\hat{\alpha}}\right)_{\hat{\alpha}}{ }^{\hat{\beta}} \nabla_{\hat{\beta} i}$.

The constraints are solved by

$$
\begin{aligned}
& \hat{\omega}_{\hat{a} \hat{b} \hat{c}}=\omega(e)_{\hat{a} \hat{b} \hat{c}}+\frac{i}{4}\left(\psi_{\hat{a} k} \Gamma_{\hat{c}} \psi_{\hat{b}}^{k}+\psi_{\hat{c} k} \Gamma_{\hat{b}} \psi_{\hat{a}}^{k}-\psi_{\hat{b} k} \Gamma_{\hat{a}} \psi_{\hat{c}}^{k}\right)+2 b_{[\hat{b}} \eta_{\hat{c}] \hat{a}}, \\
& \mathrm{i} \hat{\phi}_{\hat{m}}{ }^{i}=\frac{2}{3}\left(\Gamma^{[\hat{p}} \delta_{\hat{m}}^{\hat{q}]}+\frac{1}{4} \Gamma_{\hat{m}} \Sigma^{\hat{p} \hat{q}}\right)\left(\hat{\mathcal{D}}_{[\hat{p}} \psi_{\hat{q}]}^{i}+\frac{1}{8} w_{\hat{c} \hat{d}}\left(3 \Sigma^{\hat{c} \hat{d}} \Gamma_{[\hat{p}} \psi_{\hat{q}]}^{i}-\Gamma_{[\hat{p}} \Sigma^{\hat{c} \hat{d}} \psi_{\hat{q}]}^{i}\right)\right), \\
& \left.\hat{\mathfrak{f}}_{\hat{a}}^{\hat{b}}=-\frac{1}{6} \mathcal{R}(\hat{\omega})_{\hat{a} \hat{c}}^{\hat{b} \hat{c}}+\frac{1}{48} \delta_{\hat{a}}^{\hat{b}} \mathcal{R}(\hat{\omega})_{\hat{c} \hat{d}} \hat{d} \hat{d}-\frac{\mathrm{i}}{6} \psi_{\hat{c} j} \Gamma^{[\hat{b}} \hat{R}(Q)_{\hat{a}}{ }_{\hat{c}}\right] j-\frac{\mathrm{i}}{12} \psi_{\hat{c} j} \Gamma_{\hat{a}} \hat{R}(Q)^{\hat{b} \hat{c} j} \\
& +\frac{1}{3} \psi_{[\hat{a} j} \Sigma^{\hat{b} \hat{d}} \hat{\phi}_{\hat{d}]}^{j}-\frac{1}{24} \delta_{\hat{a}}^{\hat{b}}\left(\psi_{\hat{c} j} \Sigma^{\hat{c} \hat{d}} \hat{\phi}_{\hat{d}}^{j}\right)-\frac{2 \mathrm{i}}{3}\left(\psi_{\hat{a} j} \Gamma^{\hat{b}} \chi^{j}\right) \\
& -\frac{\mathrm{i}}{12} \psi_{\hat{a} j} \psi_{\hat{c}}^{j} w^{\hat{b} \hat{c}}+\frac{\mathrm{i}}{24}\left(\psi_{\hat{a} j} \Gamma_{\hat{e}} \psi_{\hat{d}}^{j}\right) \tilde{w}^{\hat{b} \hat{d} \hat{e}} \\
& +\frac{\mathrm{i}}{192} \delta_{\hat{a}}^{\hat{b}}\left(2\left(\psi_{\hat{c} j} \psi_{\hat{d}}^{j}\right) w^{\hat{c} \hat{d}}-\left(\psi_{\hat{c} j} \Gamma_{\hat{e}} \psi_{\hat{d}}{ }^{j}\right) \tilde{w}^{\hat{c} \hat{d} \hat{e}}\right) .
\end{aligned}
$$

One may confirm that these are equivalent to (4.29).

This redefinition dramatically simplifies many of the component curvatures. As we have already seen, $\hat{R}(P)_{\hat{a} \hat{b}}^{\hat{c}}$ vanishes. The curvature $\hat{R}(Q)_{\hat{a} \hat{b} \hat{\alpha}}^{i}$ turns out to coincide with the identically named quantity introduced in (4.21). Using the redefined spin connection, one has

$$
\hat{R}(Q)_{\hat{a} \hat{b}}^{i}=\frac{1}{2} \Pi_{\hat{a} \hat{b}} \hat{c} \hat{d}\left(\hat{\Psi}_{\hat{c} \hat{d}}^{i}-\frac{3}{4} w_{\hat{c} \hat{d}} \Gamma^{\hat{e}} \psi_{\hat{e}}^{i}-\frac{3}{4} \tilde{w}_{\hat{a} \hat{b}}^{\hat{c}} \psi_{\hat{c}}^{i}\right)
$$

The curvature $\hat{R}(D)_{\hat{a} \hat{b}}$ now vanishes while $\hat{R}(J)_{\hat{a} \hat{b}}^{i j}$ is unchanged,

$$
\hat{R}(D)_{\hat{a} \hat{b}}=0, \quad \hat{R}(J)_{\hat{a} \hat{b}}^{i j}=R(J)_{\hat{a} \hat{b}}^{i j}=-\frac{3 \mathrm{i}}{4} X_{\hat{a} \hat{b}}^{i j} \mid .
$$

The Lorentz curvature tensor $\hat{R}(M)_{\hat{a} \hat{b}} \hat{c} \hat{d}$ turns out to be simplified the most and is given, up to terms of the form $\psi \mathcal{D} \psi$ and $\psi^{2} w$, as

$$
\hat{R}(M)_{\hat{a} \hat{b}}^{\hat{c} \hat{d}}=C(\hat{\omega})_{\hat{a} \hat{b}} \hat{c} \hat{d}+(\text { explicit gravitino bilinears })
$$

where $C(\hat{\omega})_{\hat{a} \hat{b}}^{\hat{c} \hat{d}}$ is the Weyl tensor. Remarkably, from eq. (C.7e), one finds the rather simple expression

$$
\hat{R}(M)_{\hat{a} \hat{b}} \hat{b} \hat{d}=-\frac{1}{4}\left(\Sigma_{\hat{a} \hat{b}}\right)^{\hat{\alpha} \hat{\beta}}\left(\Sigma^{\hat{c} \hat{d}}\right)^{\hat{\gamma} \hat{\delta}}\left(\mathrm{i} W_{\hat{\alpha} \hat{\beta} \hat{\gamma} \hat{\delta}} \mid+3 w_{(\hat{\alpha} \hat{\beta}} w_{\hat{\gamma} \hat{\delta})}\right)
$$

defining the remaining undetermined component $W_{\hat{\alpha} \hat{\beta} \hat{\gamma} \hat{\delta}} \mid$ in terms of the new curvature $\hat{R}(M)_{\hat{a} \hat{b}} \hat{c} \hat{d}$. In practice, this is the most convenient definition of $W_{\hat{\alpha} \hat{\beta} \hat{\gamma} \hat{\delta}} \mid$.

In principle, one can construct expressions for $\hat{R}(S)_{\hat{a} \hat{b} \hat{\alpha}}$ and $\hat{R}(K)_{\hat{a} b \hat{b}} \hat{c}$ explicitly in terms of $\hat{\phi}_{\hat{m}_{\hat{\alpha}}}^{i}$ and $\hat{\mathfrak{f}}_{\hat{m}}{ }^{\hat{a}}$ in analogy with (4.31). In practice, such expressions are not terribly useful since these connections are composite quantities. Instead, we can follow the component technique of analyzing the component Bianchi identities, which in our case is equivalent to 
projecting the corresponding superspace curvatures, $\hat{R}(S)_{\hat{a} \hat{b} \hat{\alpha}}^{i}=\left.\hat{\mathscr{R}}(S)_{\hat{a} \hat{b} \hat{\alpha}}\right|^{i}$ and $\hat{R}(K)_{\hat{a} \hat{b}}^{\hat{c}}=$ $\hat{\mathscr{R}}(K)_{\hat{a} \hat{b}}^{\hat{c}} \mid$. This results in

$$
\begin{aligned}
\mathrm{i} \hat{R}(S)_{\hat{a} \hat{b}}{ }^{i}= & \hat{\nabla} \hat{R}(Q)_{\hat{a} \hat{b}}{ }^{i}+\hat{\nabla}^{\hat{c}}\left(\Gamma_{[\hat{a}} \hat{R}(Q)_{\hat{b}] \hat{c}}{ }^{i}\right)+\frac{1}{8} w_{\hat{c} \hat{d}} \sum^{\hat{c} \hat{d}} \hat{R}(Q)_{\hat{a} \hat{b}}{ }^{i}-\frac{1}{8} w^{\hat{c} \hat{d}} \sum_{\hat{a} \hat{b}} \hat{R}(Q)_{\hat{c} \hat{d}}{ }^{i} \\
& -\frac{3}{4} w^{\hat{c}} \hat{a} \hat{R}(Q)_{\hat{b}] \hat{c}}{ }^{i} \\
\hat{R}(K)_{\hat{a} \hat{b}} \hat{c}= & \frac{1}{4} \hat{\nabla}_{\hat{d}} \hat{R}(M)_{\hat{a} \hat{b}}^{\hat{c} \hat{d}}-\frac{4 \mathrm{i}}{3} \hat{R}(Q)_{\hat{a} \hat{b} j} \Gamma^{\hat{c}} \chi^{j}+\frac{\mathrm{i}}{2} \hat{R}(Q)_{\hat{d}[\hat{a} j} \Gamma_{\hat{b}]} \hat{R}(Q)^{\hat{c} \hat{d} j} \\
& -\frac{\mathrm{i}}{2} \hat{R}(Q)_{\hat{a} \hat{d} j} \Gamma^{\hat{c} \hat{R}} \hat{R}(Q)_{\hat{b}}^{\hat{d} j}
\end{aligned}
$$

It is useful to note the subsidiary relations

$$
\begin{aligned}
\mathrm{i} \Gamma^{\hat{b}} \hat{R}(S)_{\hat{a} \hat{b}}{ }^{i} & =-\frac{1}{2} \hat{\nabla} \hat{b} \hat{R}(Q)_{\hat{a} \hat{b}}{ }^{i}-\frac{5}{8} w^{\hat{b} \hat{c}} \Gamma_{\hat{b}} \hat{R}(Q)_{\hat{a} \hat{c}}{ }^{i}+\frac{1}{4} w^{\hat{b} \hat{c}} \Gamma_{\hat{a}} \hat{R}(Q)_{\hat{b} \hat{c}}{ }^{i}, \\
\mathrm{i} \Gamma^{\hat{a}} \Gamma^{\hat{b}} \hat{R}(S)_{\hat{a} \hat{b}}{ }^{i} & =0, \quad \hat{R}(K)_{\hat{a} \hat{b}} \hat{b}=0 .
\end{aligned}
$$

The $Q, S$, and $K$ transformations of the independent component fields are unchanged from (4.26), up to the redefinitions occurring above. These lead to

$$
\begin{aligned}
& \delta e_{\hat{m}}^{\hat{a}}=\mathrm{i}\left(\xi_{j} \Gamma^{\hat{a}} \psi_{\hat{m}^{j}}{ }^{j}\right), \\
& \delta \psi_{\hat{m} \hat{\alpha}}^{i}=2 \hat{\mathcal{D}}_{\hat{m}} \xi_{\hat{\alpha}}^{i}-\frac{1}{4} w_{\hat{c} \hat{d}}\left(\left(\Gamma_{\hat{m}} \Sigma^{\hat{c} \hat{d}}\right)_{\hat{\alpha}}^{\hat{\beta}}-3\left(\Sigma^{\hat{c} \hat{d}} \Gamma_{\hat{m}}\right)_{\hat{\alpha}}^{\hat{\beta}}\right) \xi_{\hat{\beta}}^{i}+2 \mathrm{i}\left(\Gamma_{\hat{m}} \eta^{i}\right)_{\hat{\alpha}}, \\
& \delta \mathcal{V}_{\hat{m}}^{i j}=3 \xi^{(i} \hat{\phi}_{\hat{m}}{ }^{j)}+8 \mathrm{i} \xi^{(i} \Gamma_{\hat{m}} \chi^{j)}-3 \eta^{(i} \psi_{\hat{m}}^{j)}, \\
& \delta b_{\hat{m}}=-\xi_{k} \hat{\phi}_{\hat{m}}{ }^{k}-\frac{8 \mathrm{i}}{3} \xi_{k} \Gamma_{\hat{m}} \chi^{k}-\eta_{k} \psi_{\hat{m}}{ }^{k}-2 e_{\hat{m}}{ }^{\hat{a}} \Lambda_{\hat{a}}, \\
& \delta w_{\hat{a} \hat{b}}=2 \mathrm{i} \xi_{i} \hat{R}(Q)_{\hat{a} \hat{b}}^{i}-\frac{32 \mathrm{i}}{3} \xi_{i} \Sigma_{\hat{a} \hat{b}} \chi^{i},
\end{aligned}
$$

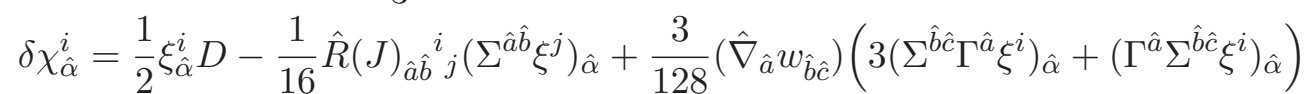

$$
\begin{aligned}
& +\frac{3}{256} w_{\hat{a} \hat{b}} w_{\hat{c} d} \varepsilon^{\hat{a} \hat{b} \hat{c} \hat{d} \hat{e}}\left(\Gamma_{\hat{e}} \xi^{i}\right)_{\hat{\alpha}}-\frac{3 \mathrm{i}}{16} w_{\hat{a} \hat{b}}\left(\Sigma^{\hat{a} \hat{b}} \eta^{i}\right)_{\hat{\alpha}}, \\
& \delta D=2 \mathrm{i} \xi_{i} \hat{\bar{\phi}} \chi^{i}+\mathrm{i} w_{\hat{a} \hat{b}}\left(\xi_{i} \Sigma^{\hat{a} \hat{b}} \chi^{i}\right)+2 \eta_{j} \chi^{j} .
\end{aligned}
$$

We emphasize that the supersymmetry transformations are equivalent to (4.26) and only the definition of the composite connections have been altered.

We have already noted the resemblance between the constraints (4.33) and those found in the existing literature. The supersymmetry transformations given above turn out to coincide very closely with those of [52], up to a field-dependent $K$-transformation. The differences with the other groups are more involved. For reference, we provide a translation table in appendix D between our conventions, employing the redefined composite connections, and those of the other groups.

\section{The covariant projective multiplets in conformal superspace}

Within the superspace approach to $\mathcal{N}=1$ supergravity in five dimensions [24-26], general supergravity-matter systems are described in terms of covariant projective multiplets. 
These are curved-superspace generalizations of the $5 \mathrm{D}$ superconformal projective multiplets [33]. In this section, the concept of covariant projective multiplets is reformulated in conformal superspace, a general procedure to generate such multiplets is given, and a universal locally supersymmetric action principle is presented.

\subsection{Covariant projective multiplets}

Let $v^{i} \in \mathbb{C}^{2} \backslash\{0\}$ denote inhomogeneous coordinates for $\mathbb{C} P^{1}$. A covariant projective multiplet of weight $n, Q^{(n)}(z, v)$, is defined to be a conformal primary Lorentz-scalar superfield, ${ }^{11}$

$$
S_{\hat{\alpha}}^{i} Q^{(n)}=0
$$

that lives on the curved superspace $\mathcal{M}^{5 \mid 8}$, is holomorphic with respect to the isospinor $v^{i}$ on an open domain of $\mathbb{C}^{2} \backslash\{0\}$, and is characterized by the following properties:

- it obeys the covariant analyticity constraint

$$
\nabla_{\hat{\alpha}}^{(1)} Q^{(n)}=0, \quad \nabla_{\hat{\alpha}}^{(1)}:=v_{i} \nabla_{\hat{\alpha}}^{i} ;
$$

- it is a homogeneous function of $v$ of degree $n$, that is,

$$
Q^{(n)}(c v)=c^{n} Q^{(n)}(v), \quad c \in \mathbb{C} \backslash\{0\} ;
$$

- the supergravity gauge transformation $(2.21)$ acts on $Q^{(n)}$ as follows:

$$
\begin{aligned}
\delta_{\mathcal{G}} Q^{(n)} & =\left(\xi^{\hat{C}} \nabla_{\hat{C}}+\Lambda^{i j} J_{i j}+\sigma \mathbb{D}\right) Q^{(n)}, \\
\Lambda^{i j} J_{i j} Q^{(n)} & =-\left(\Lambda^{(2)} \partial^{(-2)}-n \Lambda^{(0)}\right) Q^{(n)} .
\end{aligned}
$$

Here we have introduced the differential operator

$$
\partial^{(-2)}:=\frac{1}{(v, u)} u^{i} \frac{\partial}{\partial v^{i}},
$$

and also defined the parameters

$$
\Lambda^{(2)}:=\Lambda^{i j} v_{i} v_{j}, \quad \Lambda^{(0)}:=\frac{v_{i} u_{j}}{(v, u)} \Lambda^{i j}, \quad(v, u):=v^{i} u_{i} .
$$

The expressions in (5.5) and (5.6) involve a second isospinor $u^{i}$ which is subject to the condition $(v, u) \neq 0$, but otherwise it is completely arbitrary. The isospinors $v^{i}$ and $u^{i}$ are defined to be inert under the action of the supergravity gauge group. For later use, in addition to (5.5), we also introduce the operators

$$
\partial^{(2)}:=(v, u) v^{i} \frac{\partial}{\partial u^{i}}, \quad \partial^{(0)}:=v^{i} \frac{\partial}{\partial v^{i}}-u^{i} \frac{\partial}{\partial u^{i}},
$$

such that

$$
\left[\partial^{(0)}, \partial^{( \pm 2)}\right]= \pm 2 \partial^{( \pm 2)}, \quad\left[\partial^{(2)}, \partial^{(-2)}\right]=\partial^{(0)} .
$$

\footnotetext{
${ }^{11}$ As a rule, we will not indicate the $z$-dependence of $Q^{(n)}(z, v)$.
} 
By construction, the superfield $Q^{(n)}$ is independent of $u$, i.e. $\partial Q^{(n)} / \partial u^{i}=0$. It is not difficult to check that the variation $\delta_{\mathcal{G}} Q^{(n)}$ defined by (5.4) is characterized by the same property, $\partial\left(\delta_{\mathcal{G}} Q^{(n)}\right) / \partial u^{i}=0$, due to (5.3).

Since the spinor covariant derivatives satisfy

$$
\left\{\nabla_{\hat{\alpha}}^{(i}, \nabla_{\hat{\beta}}^{j)}\right\}=0 \quad \Longleftrightarrow \quad\left\{\nabla_{\hat{\alpha}}^{(1)}, \nabla_{\hat{\beta}}^{(1)}\right\}=0,
$$

the analyticity constraint (5.2) is clearly consistent with the algebra of covariant derivatives. However, we still need to check whether the conformal primary constraint on $Q^{(n)}$, $S_{\hat{\alpha}}^{i} Q^{(n)}=0$, and the analyticity constraint, $\nabla_{\hat{\alpha}}^{(1)} Q^{(n)}=0$, are mutually consistent. In complete analogy with the $4 \mathrm{D} \mathcal{N}=2$ supergravity analysis of $[55,56]$, the constraints $S_{\hat{\alpha}}^{i} Q^{(n)}=0$ and $\nabla_{\hat{\alpha}}^{(1)} Q^{(n)}=0$ lead to the integrability condition

$$
0=\left\{S_{\hat{\alpha}}^{i}, \nabla_{\hat{\beta}}^{(1)}\right\} Q^{(n)}=v_{j}\left(2 \varepsilon_{\hat{\alpha} \hat{\beta}} \varepsilon^{i j} \mathbb{D}+6 \varepsilon_{\hat{\alpha} \hat{\beta}} J^{i j}\right) Q^{(n)}=\varepsilon_{\hat{\alpha} \hat{\beta}} v^{i}(2 \mathbb{D}-3 n) Q^{(n)},
$$

which uniquely fixes the dimension of $Q^{(n)}$ to be [26]

$$
\mathbb{D} Q^{(n)}=\frac{3 n}{2} Q^{(n)} .
$$

The above definition of the covariant projective multiplets may be generalized by removing the constraint $S_{\hat{\alpha}}^{i} Q^{(n)}=0 .{ }^{12}$ For instance, given a non-primary scalar $\Phi$, the superfield $\Psi^{(4)}:=\Delta^{(4)} \Phi$ is non-primary and analytic, $\nabla_{\hat{\alpha}}^{(1)} \Psi^{(4)}=0$, with the operator $\Delta^{(4)}$ defined by (5.21c).

The analyticity constraint (5.2) and the homogeneity condition (5.3) are consistent with the interpretation that the isospinor $v^{i} \in \mathbb{C}^{2} \backslash\{0\}$ is defined modulo the equivalence relation $v^{i} \sim c v^{i}$, with $c \in \mathbb{C} \backslash\{0\}$, hence it parametrizes $\mathbb{C} P^{1}$. Therefore, the projective multiplets live in $\mathcal{M}^{5 \mid 8} \times \mathbb{C} P^{1}$, a curved five-dimensional analog of the $4 \mathrm{D} \mathcal{N}=2$ projective superspace $\mathbb{R}^{4 \mid 8} \times \mathbb{C} P^{1}[35-37]^{13}$

There exists a real structure on the space of projective multiplets. Given a weight- $n$ projective multiplet $Q^{(n)}\left(v^{i}\right)$, its smile conjugate $\breve{Q}^{(n)}\left(v^{i}\right)$ is defined by

$$
Q^{(n)}\left(v^{i}\right) \longrightarrow \bar{Q}^{(n)}\left(\bar{v}_{i}\right) \longrightarrow \bar{Q}^{(n)}\left(\bar{v}_{i} \rightarrow-v_{i}\right)=: \breve{Q}^{(n)}\left(v^{i}\right),
$$

with $\bar{Q}^{(n)}\left(\bar{v}_{i}\right):=\overline{Q^{(n)}\left(v^{i}\right)}$ the complex conjugate of $Q^{(n)}\left(v^{i}\right)$, and $\bar{v}_{i}$ the complex conjugate of $v^{i}$. One can show that $\breve{Q}^{(n)}(v)$ is a weight-n projective multiplet. In particular, $\breve{Q}^{(n)}(v)$ obeys the analyticity constraint $\nabla_{\hat{\alpha}}^{(1)} \breve{Q}^{(n)}=0$, unlike the complex conjugate of $Q^{(n)}(v)$. One can also check that

$$
\breve{Q}^{(n)}(v)=(-1)^{n} Q^{(n)}(v) .
$$

Therefore, if $n$ is even, one can define real projective multiplets, which are constrained by $\breve{Q}^{(2 n)}=Q^{(2 n)}$. Note that geometrically, the smile-conjugation is complex conjugation composed with the antipodal map on the projective space $\mathbb{C} P^{1}$.

\footnotetext{
${ }^{12}$ Non-primary projective multiplets, which possess inhomogeneous super Weyl transformation laws, naturally occur within the $\mathrm{SU}(2)$ superspace approach [26].

${ }^{13}$ The superspace $\mathbb{R}^{4 \mid 8} \times \mathbb{C} P^{1}$ was introduced for the first time by Rosly [57]. The same superspace is at the heart of the harmonic $[58,59]$ and projective [35-37] superspace approaches.
} 
We now list some projective multiplets that can be used to describe superfield dynamical variables. ${ }^{14}$ A complex $\mathcal{O}(m)$ multiplet, with $m=1,2, \ldots$, is described by a weight- $m$ projective superfield $H^{(m)}(v)$ of the form:

$$
H^{(m)}(v)=H^{i_{1} \ldots i_{m}} v_{i_{1}} \ldots v_{i_{m}} .
$$

The analyticity constraint (5.2) is equivalent to

$$
\nabla_{\hat{\alpha}}^{\left(i_{1}\right.} H^{\left.i_{2} \ldots i_{m+1}\right)}=0 .
$$

If $m$ is even, $m=2 n$, we can define a real $\mathcal{O}(2 n)$ multiplet obeying the reality condition $\breve{H}^{(2 n)}=H^{(2 n)}$, or equivalently

$$
\overline{H^{i_{1} \ldots i_{2 n}}}=H_{i_{1} \ldots i_{2 n}}=\varepsilon_{i_{1} j_{1}} \cdots \varepsilon_{i_{2 n} j_{2 n}} H^{j_{1} \ldots j_{2 n}} .
$$

For $n>1$, the real $\mathcal{O}(2 n)$ multiplet can be used to describe an off-shell (neutral) hypermultiplet.

The $\mathcal{O}(m)$ multiplets, $H^{(m)}(v)$, are well defined on the entire projective space $\mathbb{C} P^{1}$. There also exist important projective multiplets that are defined only on an open domain of $\mathbb{C} P^{1}$. Before introducing them, let us give a few definitions. We define the north chart of $\mathbb{C} P^{1}$ to consist of those points for which the first component of $v^{i}=\left(v^{\underline{1}}, v^{\underline{2}}\right)$ is non-zero, $v^{\underline{1}} \neq 0$. The north chart may be parametrized by the complex inhomogeneous coordinate $\zeta=v^{\underline{2}} / v^{\underline{1}} \in \mathbb{C}$. The only point of $\mathbb{C} P^{1}$ outside the north chart is characterized by $v_{\infty}^{i}=$ $\left(0, v^{2}\right)$ and describes an infinitely separated point. Thus we may think of the projective space $\mathbb{C} P^{1}$ as $\mathbb{C} P^{1}=\mathbb{C} \cup\{\infty\}$. The south chart of $\mathbb{C} P^{1}$ is defined to consist of those points for which the second component of $v^{i}=\left(v^{\underline{1}}, v^{2}\right)$ is non-zero, $v^{\underline{2}} \neq 0$. The south chart is naturally parametrized by $1 / \zeta$. The intersection of the north and south charts is $\mathbb{C} \backslash\{0\}$.

An off-shell (charged) hypermultiplet can be described in terms of the so-called arctic weight- $n$ multiplet $\Upsilon^{(n)}(v)$ which is defined to be holomorphic in the north chart $\mathbb{C} P^{1}$ :

$$
\Upsilon^{(n)}(v)=\left(v^{\underline{1}}\right)^{n} \Upsilon^{[n]}(\zeta), \quad \Upsilon^{[n]}(\zeta)=\sum_{k=0}^{\infty} \Upsilon_{k} \zeta^{k}
$$

Its smile-conjugate antarctic multiplet $\breve{\Upsilon}^{(n)}(v)$, has the explicit form

$$
\breve{\Upsilon}^{(n)}(v)=\left(v^{\underline{2}}\right)^{n} \breve{\Upsilon}^{[n]}(\zeta)=\left(v^{\underline{1}} \zeta\right)^{n} \breve{\Upsilon}^{[n]}(\zeta), \quad \breve{\Upsilon}^{[n]}(\zeta)=\sum_{k=0}^{\infty} \bar{\Upsilon}_{k} \frac{(-1)^{k}}{\zeta^{k}}
$$

and is holomorphic in the south chart of $\mathbb{C} P^{1}$. The arctic multiplet can be coupled to a Yang-Mills multiplet in a complex representation of the group $G_{\mathrm{YM}}$. The pair consisting of $\Upsilon^{[n]}(\zeta)$ and $\breve{\Upsilon}^{[n]}(\zeta)$ constitutes the so-called polar weight- $n$ multiplet.

Our last example is a real tropical multiplet $\mathcal{U}^{(2 n)}(v)$ of weight $2 n$ defined by

$$
\mathcal{U}^{(2 n)}(v)=\left(\mathrm{i} v^{1} v^{2}\right)^{n} \mathcal{U}^{[2 n]}(\zeta)=\left(v^{1}\right)^{2 n}(\mathrm{i} \zeta)^{n} \mathcal{U}^{[2 n]}(\zeta)
$$

\footnotetext{
${ }^{14}$ In $4 \mathrm{D} \mathcal{N}=2$ Poincaré supersymmetry, the modern terminology for projective multiplets was introduced in $[60]$.
} 


$$
\mathcal{U}^{[2 n]}(\zeta)=\sum_{k=-\infty}^{\infty} \mathcal{U}_{k} \zeta^{k}, \quad \overline{\mathcal{U}}_{k}=(-1)^{k} \mathcal{U}_{-k}
$$

This multiplet is holomorphic in the intersection of the north and south charts of the projective space $\mathbb{C} P^{1}$.

\subsection{Analytic projection operator}

In this subsection we show how to engineer covariant projective multiplets by making use of an analytic projection operator.

Let us start with a simple observation. Due to (5.9), the spinor covariant derivatives satisfy

$$
\nabla_{\hat{\alpha}}^{(i} \nabla_{\hat{\beta}}^{j} \nabla_{\hat{\gamma}}^{k} \nabla_{\hat{\delta}}^{l} \nabla_{\hat{\rho}}^{p)}=\nabla_{[\hat{\alpha}}^{(i} \nabla_{\hat{\beta}}^{j} \nabla_{\hat{\gamma}}^{k} \nabla_{\hat{\delta}}^{l} \nabla_{\hat{\rho}]}^{p)}=0 \quad \Longleftrightarrow \quad \nabla_{\hat{\alpha}}^{(1)} \nabla_{\hat{\beta}}^{(1)} \nabla_{\hat{\gamma}}^{(1)} \nabla_{\hat{\delta}}^{(1)} \nabla_{\hat{\rho}}^{(1)}=0 .
$$

Hence, if we define the operators

$$
\begin{aligned}
\Delta^{i j k l} & :=-\frac{1}{96} \varepsilon^{\hat{\alpha} \hat{\gamma} \hat{\gamma} \hat{\delta}} \nabla_{\hat{\alpha}}^{(i} \nabla_{\hat{\beta}}^{j} \nabla_{\hat{\gamma}}^{k} \nabla_{\hat{\delta}}^{l)}=-\frac{1}{32} \nabla^{(i j} \nabla^{k l)}=\Delta^{(i j k l)}, \\
\nabla^{i j} & :=\nabla^{\hat{\alpha} i} \nabla_{\hat{\alpha}}^{j}=\nabla^{(i j)}, \quad \nabla^{(2)}:=\nabla^{\hat{\alpha}(1)} \nabla_{\hat{\alpha}}^{(1)}, \\
\Delta^{(4)} & :=v_{i} v_{j} v_{k} v_{l} \Delta^{i j k l}=-\frac{1}{32}\left(\nabla^{(2)}\right)^{2},
\end{aligned}
$$

it clearly holds that

$$
\nabla_{\hat{\alpha}}^{(1)} \Delta^{(4)}=\Delta^{(4)} \nabla_{\hat{\alpha}}^{(1)}=0 .
$$

One may prove that $\Delta^{i j k l}$ satisfies the relations

$$
\nabla_{\hat{\alpha}}^{p} \Delta^{i j k l}=\frac{4}{5} \varepsilon^{p(i} \nabla_{\hat{\alpha} q} \Delta^{j k l) q}, \quad \Delta^{i j k l} \nabla_{\hat{\alpha}}^{p}=\frac{4}{5} \varepsilon^{p(i} \Delta^{j k l) q} \nabla_{\hat{\alpha} q} .
$$

The operator $\Delta^{(4)}$ is called the analytic projection operator. Given any superfield $U$, the superfield $Q:=\Delta^{(4)} U$ satisfies the analyticity condition (5.2). On the other hand, in order for $Q$ to be a covariant projective superfield, $U$ has to be constrained. In [26] it was proven in $\mathrm{SU}(2)$ superspace that the right prepotential for a covariant weight- $n$ projective superfield is an isotwistor superfield of weight $(n-4) .{ }^{15}$

By definition, a weight- $n$ isotwistor superfield $U^{(n)}$ is a primary tensor superfield (with suppressed Lorentz indices) that lives on $\mathcal{M}^{5 \mid 8}$, is holomorphic with respect to the isospinor variables $v^{i}$ on an open domain of $\mathbb{C}^{2} \backslash\{0\}$, is a homogeneous function of $v^{i}$ of degree $n$,

$$
U^{(n)}(c v)=c^{n} U^{(n)}(v), \quad c \in \mathbb{C} \backslash\{0\},
$$

and is characterized by the supergravity gauge transformation

$$
\begin{aligned}
& \delta_{\mathcal{G}} U^{(n)}=\left(\xi^{\hat{C}} \nabla_{\hat{C}}+\frac{1}{2} \Lambda^{\hat{a} \hat{b}} M_{\hat{a} \hat{b}}+\Lambda^{i j} J_{i j}+\sigma \mathbb{D}\right) U^{(n)}, \\
& J_{i j} U^{(n)}=-\left(v_{(i} v_{j} \partial^{(-2)}-\frac{n}{(v, u)} v_{(i} u_{j}\right) U^{(n)} .
\end{aligned}
$$

\footnotetext{
${ }^{15}$ The concept of isotwistor superfields was introduced in the context of $4 \mathrm{D} \mathcal{N}=2$ supergravity [61].
} 
It is clear that any weight- $n$ projective multiplet is an isotwistor superfield, but not vice versa. The main property in the definition of isotwistor superfields is their transformation rules under $\mathrm{SU}(2)$. In principle, the definition could be extended to consider non-primary superfields.

Let $U^{(n-4)}$ be a Lorentz-scalar isotwistor such that

$$
\mathbb{D} U^{(n-4)}=\frac{1}{2}(3 n-4) U^{(n-4)} .
$$

Then the weight- $n$ isotwistor superfield

$$
Q^{(n)}:=\Delta^{(4)} U^{(n-4)}
$$

satisfies all the properties of a covariant projective multiplet. Note that $Q^{(n)}$ is clearly analytic with $\mathbb{D} Q^{(n)}=\frac{3 n}{2} Q^{(n)}$. It is an instructive exercise to check that $Q^{(n)}$ is primary. We define the operators

$$
S_{\hat{\alpha}}^{(1)}:=v_{i} S_{\hat{\alpha}}^{i}, \quad S_{\hat{\alpha}}^{(-1)}:=\frac{u_{i}}{(v, u)} S_{\hat{\alpha}}^{i}
$$

which satisfy

$$
\left\{S_{\hat{\alpha}}^{(1)}, \nabla_{\hat{\beta}}^{(1)}\right\}=6 \varepsilon_{\hat{\alpha} \hat{\beta}} J^{(2)}, \quad\left\{S_{\hat{\alpha}}^{(-1)}, \nabla_{\hat{\beta}}^{(1)}\right\}=2 \varepsilon_{\hat{\alpha} \hat{\beta}} \mathbb{D}-4 M_{\hat{\alpha} \hat{\beta}}+6 \varepsilon_{\hat{\alpha} \hat{\beta}} J^{(0)},
$$

where

$$
\begin{array}{rlrl}
J^{(2)}:=v_{i} v_{j} J^{i j}, & {\left[J^{(2)}, \nabla_{\hat{\alpha}}^{(1)}\right]=0,} & J^{(2)} U^{(n)} & =0, \\
J^{(0)}:=\frac{v_{i} u_{j}}{(v, u)} J^{i j}, & {\left[J^{(0)}, \nabla_{\hat{\alpha}}^{(1)}\right]=-\frac{1}{2} \nabla_{\hat{\alpha}}^{(1)},} & J^{(0)} U^{(n)}=-\frac{n}{2} U^{(n)} .
\end{array}
$$

After some algebra, it can be proven that

$$
\begin{aligned}
{\left[S_{\hat{\rho}}^{(1)}, \Delta^{(4)}\right] } & =-\frac{1}{4} \varepsilon^{\hat{\alpha} \hat{\beta} \hat{\gamma} \hat{\delta}} \varepsilon_{\hat{\rho} \hat{\alpha}} \nabla_{\hat{\beta}}^{(1)} \nabla_{\hat{\gamma}}^{(1)} \nabla_{\hat{\delta}}^{(1)} J^{(2)}, \\
{\left[S_{\hat{\rho}}^{(-1)}, \Delta^{(4)}\right] } & =\frac{1}{24} \varepsilon^{\hat{\alpha} \hat{\beta} \hat{\gamma} \hat{\delta}} \nabla_{\hat{\beta}}^{(1)} \nabla_{\hat{\gamma}}^{(1)} \nabla_{\hat{\delta}}^{(1)}\left[\varepsilon_{\hat{\rho} \hat{\alpha}}\left(8-2 \mathbb{D}-6 J^{(0)}\right)+4 M_{\hat{\rho} \hat{\alpha}}\right] .
\end{aligned}
$$

Using these results, it immediately follows that $S_{\hat{\alpha}}^{i} \Delta^{(4)} U^{(n)}=0$.

Let us conclude this subsection by giving the expression for $\Delta^{(4)}$ in $\mathrm{SU}(2)$ superspace. This can be computed by simply using the degauging procedure developed in section 3 . The result is

$$
\begin{aligned}
\Delta^{(4)} U^{(n-4)}= & -\frac{1}{32} \mathcal{D}^{(2)} \mathcal{D}^{(2)} U^{(n-4)}+\frac{1}{32} \mathcal{D}^{(2)}\left(\mathfrak{F}^{(2) \hat{\alpha} \hat{\beta}}\left\{S_{\hat{\beta}}^{(-1)}, \nabla_{\hat{\alpha}}^{(1)}\right\} U^{(n-4)}\right) \\
& +\frac{1}{32} \mathcal{D}^{\hat{\alpha}(1)}\left(\mathfrak{F}_{\hat{\alpha}}^{(2) \hat{\beta}}\left[S_{\hat{\beta}}^{(-1)}, \nabla^{(2)}\right] U^{(n-4)}\right) \\
& +\frac{1}{32} \mathfrak{F}^{(2) \hat{\alpha} \hat{\beta}}\left\{S_{\hat{\beta}}^{(-1)}, \nabla_{\hat{\alpha}}^{(1)} \nabla^{(2)}\right\} U^{(n-4)},
\end{aligned}
$$

with

$$
\mathcal{D}_{\hat{\alpha}}^{(1)}:=v_{i} \mathcal{D}_{\hat{\alpha}}^{i}, \quad \mathcal{D}^{(2)}:=\mathcal{D}^{(1) \hat{\alpha}} \mathcal{D}_{\hat{\alpha}}^{(1)}, \quad \mathfrak{F}_{\hat{\alpha} \hat{\beta}}^{(2)}:=v_{i} v_{j} \mathfrak{F}_{\hat{\alpha} \hat{\beta}}^{i j}
$$


Computing the (anti-)commutators involving $S_{\hat{\beta}}^{(-1)}$ in (5.31) produces new terms involving $\nabla$, which have to be degauged. Finally, making use of the identities

$$
\begin{aligned}
\mathcal{D}^{(4)}:= & -\frac{1}{96} \varepsilon^{\hat{\alpha} \hat{\beta} \hat{\gamma} \hat{\delta}} \mathcal{D}_{\hat{\alpha}}^{(1)} \mathcal{D}_{\hat{\beta}}^{(1)} \mathcal{D}_{\hat{\gamma}}^{(1)} \mathcal{D}_{\hat{\delta}}^{(1)}, \\
\mathcal{D}^{(4)}= & -\frac{1}{96}\left[3 \mathcal{D}^{(2)} \mathcal{D}^{(2)}+24\left(\mathcal{D}^{\hat{\alpha}(1)} \mathfrak{F}_{\hat{\alpha} \hat{\beta}}^{(2)}\right) \mathcal{D}^{\hat{\beta}(1)}+4\left(\mathcal{D}^{\hat{\alpha}(1)} \mathfrak{F}^{(2) \hat{\beta}}{ }_{\hat{\beta}}\right) \mathcal{D}_{\hat{\alpha}}^{(1)}\right. \\
& \left.\quad+24 \mathfrak{F}_{\hat{\alpha} \hat{\beta}}^{(2)} \mathcal{D}^{\hat{\alpha}(1)} \mathcal{D}^{\hat{\beta}(1)}+4 \mathfrak{F}^{(2) \hat{\beta}} \mathcal{D}^{(2)}\right] U^{(n-4)}, \\
\mathcal{D}_{\hat{\alpha}}^{(1)} \mathfrak{F}_{\hat{\beta} \hat{\gamma}}^{(2)}= & \mathcal{D}_{[\hat{\alpha}}^{(1)} \mathfrak{F}_{\hat{\beta} \hat{\gamma}]}^{(2)}, \\
\mathcal{D}_{\hat{\alpha}}^{(1)} S^{(2)}= & \frac{1}{10} \mathcal{D}^{\hat{\beta}(1)} C_{\hat{\alpha} \hat{\beta}}^{(2)}, \quad S^{(2)}:=v_{i} v_{j} S^{i j}, \quad C_{\hat{a}}^{(2)}:=v_{i} v_{j} C_{\hat{a}}^{i j},
\end{aligned}
$$

we obtain

$$
\begin{aligned}
\Delta^{(4)} U^{(n-4)}= & {\left[\mathcal{D}^{(4)}-\frac{5 \mathrm{i}}{12} S^{(2)} \mathcal{D}^{(2)}-\frac{\mathrm{i}}{8} C^{\hat{\alpha} \hat{\beta}(2)} \mathcal{D}_{\hat{\alpha}}^{(1)} \mathcal{D}_{\hat{\beta}}^{(1)}-\frac{\mathrm{i}}{6}\left(\mathcal{D}^{\hat{\alpha}(1)} C_{\hat{\alpha} \hat{\beta}}^{(2)}\right) \mathcal{D}^{\hat{\beta}(1)}\right.} \\
& \left.-\frac{\mathrm{i}}{20}\left(\mathcal{D}^{\hat{\alpha}(1)} \mathcal{D}^{\hat{\beta}(1)} C_{\hat{\alpha} \hat{\beta}}^{(2)}\right)+3\left(S^{(2)}\right)^{2}+\frac{1}{4} C^{\hat{a}(2)} C_{\hat{a}}^{(2)}\right] U^{(n-4)}
\end{aligned}
$$

This relation determines the analytic projection operator in $\mathrm{SU}(2)$ superspace, which is a new result. In [26], this operator was computed only in a super Weyl gauge in which $C_{\hat{a}}^{i j}=0$.

\subsection{The action principle}

We turn to re-formulating the supersymmetric action principle given in [26] in conformal superspace.

Consider a Lagrangian $\mathcal{L}^{(2)}$ chosen to be a real weight-2 projective multiplet. Associated with $\mathcal{L}^{(2)}$ is the action ${ }^{16}$

$$
S\left[\mathcal{L}^{(2)}\right]=\frac{1}{2 \pi} \oint_{\gamma}(v, \mathrm{~d} v) \int \mathrm{d}^{5 \mid 8} z E C^{(-4)} \mathcal{L}^{(2)} .
$$

Here the superfield $C^{(-4)}$ is required to be a Lorentz-scalar primary isotwistor superfield of weight -4 such that the following two conditions hold:

$$
\Delta^{(4)} C^{(-4)}=1, \quad \mathbb{D} C^{(-4)}=-2 C^{(-4)} .
$$

These conditions prove to guarantee that the action (5.35) is invariant under the full supergravity gauge group $\mathcal{G}$. The invariance of $S\left[\mathcal{L}^{(2)}\right]$ under the Lorentz and special conformal transformations is obvious, since all the superfields in the action are Lorentz-scalar primary superfields. Invariance under the general coordinate transformations is also trivial, while invariance under the $\mathrm{SU}(2)$ transformations can be shown in complete analogy with the

\footnotetext{
${ }^{16}$ In parallel with the construction in four dimensions [56], it is possible to integrate out half of the Grassmann coordinates thus representing the action as an integral of $\mathcal{L}^{(2)}$ over an analytic subspace. (This is analogous to the chiral integral in $4 \mathrm{D} \mathcal{N}=1$ supergravity, see [62-64] for reviews.) We find it more convenient to employ the superfield $C^{(-4)}$ to always deal with full superspace integrals.
} 
proof given in $[25,26]$. It remains to prove that the action is invariant under dilatations. This simply follows from the observation that the measure, $E$, has dimension -1 .

All information about a dynamical system is encoded in its Lagrangian $\mathcal{L}^{(2)}$. The important point is that the action (5.35) does not depend on $C^{(-4)}$ if the Lagrangian $\mathcal{L}^{(2)}$ is independent of $C^{(-4)}$. To prove this statement, let us represent the Lagrangian as $\mathcal{L}^{(2)}=\Delta^{(4)} \mathcal{U}^{(-2)}$, for some isotwistor superfield $\mathcal{U}^{(-2)}$ of weight -2 . We note that for any pair of Lorentz-scalar isotwistor superfields $\Phi^{(n-4)}$ and $\Psi^{(-n-2)}$ such that

$$
\mathbb{D} \Phi^{(n-4)}=\frac{1}{2}(3 n-4) \Phi^{(n-4)}, \quad \mathbb{D} \Psi^{(-n-2)}=\frac{1}{2}(2-3 n) \Psi^{(-n-2)},
$$

we can use integration by parts to prove the following relation

$$
\oint_{\gamma}(v, \mathrm{~d} v) \int \mathrm{d}^{5 \mid 8} z E\left\{\Phi^{(n-4)} \Delta^{(4)} \Psi^{(-n-2)}-\Psi^{(-n-2)} \Delta^{(4)} \Phi^{(n-4)}\right\}=0 .
$$

If we use this result and eq. (5.36), we can rewrite the action in the form

$$
S=\frac{1}{2 \pi} \oint_{\gamma}(v, \mathrm{~d} v) \int \mathrm{d}^{5 \mid 8} z E \mathcal{U}^{(-2)}
$$

This representation makes manifest the fact that the action does not depend on $C^{(-4)}$. Upon degauging to $\mathrm{SU}(2)$ superspace, the action (5.39) coincides with the one given in [26].

A natural choice for $C^{(-4)}$ is available if the theory under consideration possesses an abelian vector multiplet such that its field strength $W$ is nowhere vanishing. Given $W$, we can construct a composite $\mathcal{O}(2)$ projective multiplet as

$$
H_{\mathrm{VM}}^{(2)}=\frac{\mathrm{i}}{2} W \nabla^{(2)} W+\mathrm{i}\left(\nabla^{\hat{\alpha}(1)} W\right) \nabla_{\hat{\alpha}}^{(1)} W=v_{i} v_{j} H_{\mathrm{VM}}^{i j},
$$

where $H_{\mathrm{VM}}^{i j}$ coincides with eq. (2.54) for a single abelian vector multiplet. By using the Bianchi identity (2.49), which implies

$$
\nabla_{\hat{\alpha}}^{(1)} \nabla_{\hat{\beta}}^{(1)} W=\frac{1}{4} \varepsilon_{\hat{\alpha} \hat{\beta}} \nabla^{(2)} W, \quad \nabla_{\hat{\alpha}}^{(1)} \nabla_{\hat{\beta}}^{(1)} \nabla_{\hat{\gamma}}^{(1)} W=0,
$$

it is a simple exercise to show that $H_{\mathrm{VM}}^{(2)}$ is an analytic superfield, $\nabla_{\hat{\alpha}}^{(1)} H_{\mathrm{VM}}^{(2)}=0$. By using (5.28), it also simple to show that $S_{\hat{\alpha}}^{(1)} H_{\mathrm{VM}}^{(2)}=S_{\hat{\alpha}}^{(-1)} H_{\mathrm{VM}}^{(2)}=0$ and then $H_{\mathrm{VM}}^{(2)}$ is primary, $S_{\hat{\alpha}}^{i} H_{\mathrm{VM}}^{(2)}=0$. We can then introduce

$$
C^{(-4)}=\frac{4 W^{4}}{3\left(H_{\mathrm{VM}}^{(2)}\right)^{2}},
$$

which consistently defines a weight -4 isotwistor superfield that, due to

$$
\Delta^{(4)} W^{4}=\frac{3}{4}\left(H_{\mathrm{VM}}^{(2)}\right)^{2},
$$

satisfies $\Delta^{(4)} C^{(-4)}=1$. The resulting action principle takes the form

$$
S\left[\mathcal{L}^{(2)}\right]=\frac{2}{3 \pi} \oint(v, \mathrm{~d} v) \int \mathrm{d}^{5 \mid 8} z E \frac{\mathcal{L}^{(2)} W^{4}}{\left(H_{\mathrm{VM}}^{(2)}\right)^{2}} .
$$


Upon degauging to $\mathrm{SU}(2)$ superspace, this action reduces to the one proposed in [26].

We conclude this section by mentioning that the action (5.35) is characterized by the following important property:

$$
S\left[G^{(2)}(\lambda+\breve{\lambda})\right]=0,
$$

with $G^{(2)}$ a real $\mathcal{O}(2)$ multiplet and $\lambda$ an arctic weight-zero multiplet. Since $\lambda$ is arbitrary, the above relation is equivalent to

$$
S\left[G^{(2)} \lambda\right]=0 .
$$

A proof of (5.45) will be given in section 7.4.

\section{Prepotentials for the vector multiplet}

In this section we develop a prepotential formulation for the Yang-Mills multiplet introduced in section 2.6. Our presentation is very similar to that given in [65] in the case of 3D $\mathcal{N}=4$ conformal supergravity. The latter was inspired by the pioneer works of Lindström and Roček [37] and Zupnik [66] devoted to the 4D $\mathcal{N}=2$ super Yang-Mills theory.

\subsection{Tropical prepotential}

The Yang-Mills multiplet in conformal superspace has been described in section 2.6. The field strength $\boldsymbol{W}$ appears in the anti-commutator of two spinor covariant derivatives as

$$
\left\{\nabla_{\hat{\alpha}}^{i}, \nabla_{\hat{\beta}}^{j}\right\}=\cdots+2 \varepsilon_{\hat{\alpha} \hat{\beta}} \varepsilon^{i j} \boldsymbol{W},
$$

where the ellipsis stands for the purely supergravity part. Let us introduce the gauge covariant operators

$$
\nabla_{\hat{\alpha}}^{(1)}:=v_{i} \nabla_{\hat{\alpha}}^{i}
$$

It may be seen that they strictly anti-commute with each other,

$$
\left\{\boldsymbol{\nabla}_{\hat{\alpha}}^{(1)}, \nabla_{\hat{\beta}}^{(1)}\right\}=0 .
$$

This means that $\nabla_{\hat{\alpha}}^{(1)}$ may be represented in the form:

$$
\boldsymbol{\nabla}_{\hat{\alpha}}^{(1)}=\mathrm{e}^{\boldsymbol{\Omega}_{+}} \nabla_{\hat{\alpha}}^{(1)} \mathrm{e}^{-\boldsymbol{\Omega}_{+}},
$$

where $\boldsymbol{\Omega}_{+}$denotes a Lie-algebra-valued bridge superfield of the form

$$
\boldsymbol{\Omega}_{+}(v)=\boldsymbol{\Omega}_{+}(\zeta)=\sum_{n=0}^{\infty} \boldsymbol{\Omega}_{n} \zeta^{n}, \quad \zeta:=\frac{v^{\underline{2}}}{v^{\underline{1}}}
$$

The bridge is a covariant weight-0 isotwistor superfield. Another representation for $\nabla_{\hat{\alpha}}^{(1)}$ follows by applying the smile-conjugation to (6.4). The result is

$$
\boldsymbol{\nabla}_{\hat{\alpha}}^{(1)}=\mathrm{e}^{-\boldsymbol{\Omega}_{-}} \nabla_{\hat{\alpha}}^{(1)} \mathrm{e}^{\boldsymbol{\Omega}_{-}}, \quad \boldsymbol{\Omega}_{-}(v)=\boldsymbol{\Omega}_{-}(\zeta)=\sum_{n=0}^{\infty}(-1)^{n} \boldsymbol{\Omega}_{n}^{\dagger} \frac{1}{\zeta^{n}} .
$$


We now introduce a Lie algebra-valued superfield $\boldsymbol{V}(\zeta)$ defined by

$$
\mathrm{e}^{\boldsymbol{V}}:=\mathrm{e}^{\boldsymbol{\Omega}_{-}} \mathrm{e}^{\boldsymbol{\Omega}_{+}}, \quad \boldsymbol{V}(v)=\boldsymbol{V}(\zeta)=\sum_{n=-\infty}^{\infty} \boldsymbol{V}_{n} \zeta^{n}, \quad \boldsymbol{V}_{n}^{\dagger}=(-1)^{n} \boldsymbol{V}_{-n} .
$$

It may be seen from (6.4) and (6.6) that $\boldsymbol{V}$ is a covariant weight-0 projective multiplet,

$$
\nabla_{\hat{\alpha}}^{(1)} \boldsymbol{V}=0 \text {. }
$$

In accordance with (6.4), the gauge transformation law of $\boldsymbol{\Omega}_{+}$is

$$
\mathrm{e}^{\boldsymbol{\Omega}_{+}^{\prime}(\zeta)}=\mathrm{e}^{\mathrm{i} \boldsymbol{\tau}} \mathrm{e}^{\boldsymbol{\Omega}_{+}(\zeta)} \mathrm{e}^{-\mathrm{i} \boldsymbol{\lambda}(\zeta)},
$$

where the new gauge parameter $\boldsymbol{\lambda}(\zeta)$ is a covariant weight-zero arctic multiplet

$$
\nabla_{\hat{\alpha}}^{(1)} \lambda=0, \quad \boldsymbol{\lambda}(\zeta)=\sum_{n=0}^{\infty} \boldsymbol{\lambda}_{n} \zeta^{n}
$$

The gauge transformation law of the tropical prepotential is

$$
\mathrm{e}^{\boldsymbol{V}^{\prime}}=\mathrm{e}^{\mathrm{i} \breve{\lambda}_{\mathrm{e}} \boldsymbol{V}_{\mathrm{e}}-\mathrm{i} \boldsymbol{\lambda}} .
$$

Hence $\boldsymbol{V}$ transforms under the $\lambda$-group only.

\subsection{Polar hypermultiplets}

Supersymmetric matter in arbitrary representations of the gauge group $G_{\mathrm{YM}}$ may be described in terms of gauge covariantly arctic multiplets and their smile-conjugate antarctic multiplets.

A gauge covariantly arctic multiplet of weight $n, \boldsymbol{\Upsilon}^{(n)}(v)$, is defined by

$$
\boldsymbol{\nabla}_{\hat{\alpha}}^{(1)} \boldsymbol{\Upsilon}^{(n)}=0, \quad \boldsymbol{\Upsilon}^{(n)}(v)=\left(v^{\underline{1}}\right)^{n} \sum_{k=0}^{\infty} \boldsymbol{\Upsilon}_{k} \zeta^{k}
$$

It can be represented in the form

$$
\boldsymbol{\Upsilon}^{(n)}(v)=\mathrm{e}^{\boldsymbol{\Omega}_{+}(v)} \Upsilon^{(n)}(v),
$$

where $\Upsilon^{(n)}(v)$ is an ordinary covariant arctic multiplet of weight $n$ as already introduced in eq. (5.17).

Computing the smile conjugate of $\mathbf{\Upsilon}^{(n)}(v)$ gives a gauge covariantly antarctic multiplet of weight $n, \breve{\Upsilon}^{(n)}(v)$, with the properties

$$
\breve{\Upsilon}^{(n)} \overleftarrow{\nabla_{\hat{\alpha}}^{(1)}}=0, \quad \breve{\Upsilon}^{(n)}(v)=\left(v^{2}\right)^{n} \sum_{k=0}^{\infty}(-1)^{k} \boldsymbol{\Upsilon}_{k}^{\dagger} \frac{1}{\zeta^{k}}
$$

It can be represented in the form

$$
\breve{\Upsilon}^{(n)}(v)=\breve{\Upsilon}^{(n)}(v) \mathrm{e}^{\boldsymbol{\Omega}_{-}(v)},
$$


where $\breve{\Upsilon}^{(n)}(v)$ is an ordinary antarctic multiplet as in eq. (5.18).

The arctic multiplet of weight $n, \boldsymbol{\Upsilon}^{(n)}(v)$, and its smile-conjugate, $\breve{\Upsilon}^{(n)}(v)$, constitute the polar multiplet of weight $n$. The gauge transformation laws of $\boldsymbol{\Upsilon}^{(n)}(v)$ and $\breve{\Upsilon}^{(n)}(v)$ are

$$
\boldsymbol{\Upsilon}^{(n) \prime}(v)=\mathrm{e}^{\mathrm{i} \tau} \boldsymbol{\Upsilon}^{(n)}(v), \quad \breve{\Upsilon}^{(n) \prime}(v)=\breve{\Upsilon}^{(n)}(v) \mathrm{e}^{-\mathrm{i} \boldsymbol{\tau}}
$$

The gauge transformation laws of $\Upsilon^{(n)}(v)$ and $\breve{\Upsilon}^{(n)}(v)$ are

$$
\Upsilon^{(n) \prime}(v)=\mathrm{e}^{\mathrm{i} \boldsymbol{\lambda}(v)} \Upsilon^{(n)}(v), \quad \breve{\Upsilon}^{(n) \prime}(v)=\breve{\Upsilon}^{(n)}(v) \mathrm{e}^{-\mathrm{i} \breve{\boldsymbol{\lambda}}(v)}
$$

In the $n=1$ case, a gauge invariant hypermultiplet Lagrangian can be constructed and is given by

$$
\mathcal{L}^{(2)}=\mathrm{i} \breve{\Upsilon}^{(1)} \boldsymbol{\Upsilon}^{(1)}=\mathrm{i} \breve{\Upsilon}^{(1)} \mathrm{e} \boldsymbol{V} \Upsilon^{(1)}
$$

\subsection{Arctic and antarctic representations}

Here we demonstrate that the Yang-Mills gauge connection $\boldsymbol{V}_{\hat{A}}$, eq. (2.43), may be expressed in terms of the tropical prepotential $\boldsymbol{V}(\zeta)$, modulo the $\tau$-gauge freedom.

Let us introduce the operator

$$
\nabla_{\hat{\alpha}}^{(-1)}:=\frac{1}{(v, u)} u_{i} \nabla_{\hat{\alpha}}^{i}
$$

It can be seen that

$$
\left\{\boldsymbol{\nabla}_{\hat{\alpha}}^{(1)}, \nabla_{\hat{\beta}}^{(-1)}\right\}=\cdots-2 \varepsilon_{\hat{\alpha} \hat{\beta}} \boldsymbol{W} .
$$

Here the ellipsis denotes purely supergravity terms. Note that the operators $\partial^{(2)}, \partial^{(-2)}$ and $\partial^{(0)}$ are invariant under the $\tau$-group transformations and obey

$$
\begin{array}{rlrl}
{\left[\partial^{(2)}, \nabla_{\hat{\alpha}}^{(1)}\right]} & =\left[\partial^{(-2)}, \nabla_{\hat{\alpha}}^{(-1)}\right]=0, & & \\
{\left[\partial^{(2)}, \nabla_{\hat{\alpha}}^{(-1)}\right]} & =\nabla_{\hat{\alpha}}^{(1)}, & & {\left[\partial^{(-2)}, \nabla_{\hat{\alpha}}^{(1)}\right]=\nabla_{\hat{\alpha}}^{(-1)},} \\
{\left[\partial^{(0)}, \nabla_{\hat{\alpha}}^{(1)}\right]} & =\nabla_{\hat{\alpha}}^{(1)}, & {\left[\partial^{(0)}, \nabla_{\hat{\alpha}}^{(-1)}\right]=-\nabla_{\hat{\alpha}}^{(-1)} .}
\end{array}
$$

When dealing with polar hypermultiplets, it is useful to introduce an arctic representation defined by the transformation

$$
\hat{\mathcal{O}} \rightarrow \hat{\mathcal{O}}_{+}:=\mathrm{e}^{-\boldsymbol{\Omega}_{+}} \hat{\mathcal{O}} \mathrm{e}^{\boldsymbol{\Omega}_{+}}, \quad U \rightarrow U_{+}:=\mathrm{e}^{-\boldsymbol{\Omega}_{+}} U
$$

applied to any gauge covariant operator $\hat{\mathcal{O}}$ and matter superfield $U .{ }^{17}$ In the arctic representation, any gauge covariantly arctic multiplet $\boldsymbol{\Upsilon}^{(n)}(v)$ becomes the ordinary arctic one, $\Upsilon^{(n)}(v)$

$$
\boldsymbol{\Upsilon}^{(n)}(v) \rightarrow \Upsilon^{(n)}(v), \quad \breve{\Upsilon}^{(n)}(v) \rightarrow \breve{\Upsilon}^{(n)}(v) \mathrm{e}^{\boldsymbol{V}(\zeta)},
$$

and the gauge covariant derivatives $\nabla_{\hat{\alpha}}^{(1)}$ turn into the standard ones,

$$
\nabla_{\hat{\alpha}}^{(1)} \rightarrow \nabla_{\hat{\alpha}}^{(1)}
$$

\footnotetext{
${ }^{17}$ It is assumed that the gauge transformation law of $\hat{\mathcal{O}}$ is $\hat{\mathcal{O}} \rightarrow \hat{\mathcal{O}}^{\prime}=\mathrm{e}^{\mathrm{i} \boldsymbol{\tau}} \hat{\mathcal{O}} \mathrm{e}^{-\mathrm{i} \boldsymbol{\tau}}$, while $U$ transforms as in $(2.46)$.
} 
The important point is that the projective derivative $\partial^{(-2)}$ is replaced by the operator

$$
\partial^{(-2)} \rightarrow \partial_{+}^{(-2)}:=\partial^{(-2)}+\mathrm{e}^{-\boldsymbol{\Omega}_{+}}\left(\partial^{(-2)} \mathrm{e}^{\boldsymbol{\Omega}_{+}}\right),
$$

which transforms as a covariant derivative under the $\lambda$-group. It is also important to mention that $\partial^{(2)}$ remains short in the arctic representation, $\partial_{+}^{(2)}=\partial^{(2)}$. Making use of the arctic-representation version of (6.20) as well as the relation

$$
\boldsymbol{\nabla}_{+\hat{\alpha}}^{(-1)}=\left[\partial_{+}^{(-2)}, \nabla_{\hat{\alpha}}^{(1)}\right]=\nabla_{\hat{\alpha}}^{(-1)}-\nabla_{\hat{\alpha}}^{(1)}\left(\mathrm{e}^{-\boldsymbol{\Omega}_{+}} \partial^{(-2)} \mathrm{e}^{\boldsymbol{\Omega}_{+}}\right),
$$

we read off

$$
\boldsymbol{W}_{+}=\frac{1}{8} \nabla^{(2)}\left(\mathrm{e}^{-\boldsymbol{\Omega}_{+} \partial^{(-2)}} \mathrm{e}^{\boldsymbol{\Omega}_{+}}\right) .
$$

Since $\partial^{(2)} \boldsymbol{W}_{+}=0, \boldsymbol{W}_{+}$is independent of $u^{i}$. The field strength $\boldsymbol{W}_{+}$also satisfies the property

$$
\partial_{+}^{(-2)} \boldsymbol{W}_{+}=0
$$

since in the original representation $\boldsymbol{W}$ is independent of $v^{i}$. The field strength can be seen to obey the Bianchi identity

$$
\boldsymbol{\nabla}_{+\hat{\alpha}}^{(i} \nabla_{+\hat{\beta}}^{j)} \boldsymbol{W}_{+}=\frac{1}{4} \varepsilon_{\hat{\alpha} \hat{\beta}} \nabla_{+}^{\hat{\gamma}(i} \nabla_{+\hat{\gamma}}^{j)} \boldsymbol{W}_{+} .
$$

In the case of a $\mathrm{U}(1)$ gauge group, $\boldsymbol{W}=W T$, with $T$ the $\mathrm{U}(1)$ generator, we have $W=W_{+}$and eq. (6.27) turns into

$$
W=\frac{1}{8} \nabla^{(2)} \partial^{(-2)} \Omega_{+} .
$$

Since $\Omega_{+}(v)=\Omega_{+}(\zeta)$, in the north chart of $\mathbb{C} P^{1}$ we can represent

$$
\partial^{(-2)} \Omega_{+}(v)=-\frac{1}{\left(v^{\underline{1}}\right)^{2}} \partial_{\zeta} \Omega_{+}(\zeta)
$$

Taking into account the fact that $W$ is independent of $\zeta$, it is simple to show that

$$
W=-\frac{1}{8} \nabla \underline{22} \Omega_{1}=\frac{1}{8} \nabla \frac{11}{} \Omega_{-1} .
$$

In complete analogy with the arctic representation, eq. (6.22), we can introduce the antarctic representation defined by

$$
\hat{\mathcal{O}} \rightarrow \hat{\mathcal{O}}_{-}:=\mathrm{e}^{\boldsymbol{\Omega}_{-}} \hat{\mathcal{O}} \mathrm{e}^{-\boldsymbol{\Omega}_{-}}, \quad U \rightarrow U_{-}:=\mathrm{e}^{\boldsymbol{\Omega}_{-}} U
$$

In this representation, the super Yang-Mills field strength takes the form

$$
\boldsymbol{W}_{-}=\frac{1}{8} \nabla^{(2)}\left(\mathrm{e}^{\boldsymbol{\Omega}_{-}} \partial^{(-2)} \mathrm{e}^{-\boldsymbol{\Omega}_{-}}\right) .
$$

Comparing the above with (6.27) gives

$$
\boldsymbol{W}_{-}=\mathrm{e}^{\boldsymbol{V}} \boldsymbol{W}_{+} \mathrm{e}^{-\boldsymbol{V}}
$$




\subsection{Abelian field strength: contour integral representation}

In the previous subsection, in the case of an abelian vector multiplet, we have derived the result (6.32). This expresses the field strength in terms of the bridge components. It is useful to find yet another representation given in terms of the real weight-zero tropical prepotential

$$
V=\Omega_{+}+\Omega_{-}
$$

It turns out that the expression

$$
W=-\frac{\mathrm{i}}{16 \pi} \oint(v, \mathrm{~d} v) \nabla^{(-2)} V, \quad \nabla^{(-2)}:=\frac{1}{(v, u)^{2}} u_{i} u_{j} \nabla^{i j},
$$

is equivalent to (6.32). It is instructive to prove this statement.

First of all, the expression for $W$ in $(6.37)$ can be shown to be independent of $u_{i}$. To see this, consider a shift

$$
u_{i} \rightarrow u_{i}+\delta u_{i}
$$

and represent it as

$$
\delta u_{i}=(v, u) v_{i} \alpha^{(-2)}+u_{i} \beta^{(0)}, \quad \alpha^{(-2)}=-\frac{u^{i} \delta u_{i}}{(v, u)^{2}}, \quad \beta^{(0)}=\frac{v^{i} \delta u_{i}}{(v, u)} .
$$

Then one can compute

$$
\delta W=-\frac{\mathrm{i}}{16 \pi} \oint(v, \mathrm{~d} v) \alpha^{(-2)}\left\{\nabla^{\hat{\alpha}(1)}, \nabla_{\hat{\alpha}}^{(-1)}\right\} V=0,
$$

which is identically zero since

$$
\left\{\nabla_{\hat{\alpha}}^{(1)}, \nabla_{\hat{\beta}}^{(-1)}\right\}=2 \mathrm{i} \nabla_{\hat{\alpha} \hat{\beta}}+2 \mathrm{i} \varepsilon_{\hat{\alpha} \hat{\beta}} \mathscr{W}, \quad \Longrightarrow\left\{\nabla^{\hat{\alpha}(1)}, \nabla_{\hat{\alpha}}^{(-1)}\right\}=8 \mathrm{i} \mathscr{W},
$$

and $\mathscr{W} V \equiv 0$. In the north chart of $\mathbb{C} P^{1}$ we have

$$
V(v)=V(\zeta)=\sum_{k=-\infty}^{\infty} \zeta^{k} V_{k}, \quad V_{k}=(-1)^{k} \bar{V}_{-k}
$$

Then choosing $u_{i}=(0,1)$ we can represent $W$ as follows

$$
W=\frac{\mathrm{i}}{16 \pi} \oint \frac{\mathrm{d} \zeta}{\zeta^{2}} \nabla \underline{22} V(\zeta)=-\frac{1}{8} \nabla^{22} V_{1}=-\frac{1}{8} \nabla^{\underline{22}} \Omega_{1} .
$$

The last expression is clearly equivalent to (6.32). Note that, due to the analysis of the previous subsection, this equivalence also guarantees that $W$ defined by (6.37) is a primary superfield, $S_{\hat{\alpha}}^{i} W=0$, satisfies the Bianchi identity $\nabla_{\hat{\alpha}}^{(1)} \nabla_{\hat{\beta}}^{(1)} W=\frac{1}{4} \varepsilon_{\hat{\alpha} \hat{\beta}} \nabla^{(2)} W$, and is invariant under the $\lambda$-group transformations

$$
\delta V=\lambda+\breve{\lambda}
$$

All these properties can actually be directly proven by using the integral representation (6.37). 


\subsection{Mezincescu's prepotential}

According to the analysis of section 5.2, we can solve the analyticity constraint on the projective prepotential $V(v)$ in terms of a primary real isotwistor superfield $V^{(-4)}(v)$ of weight $(-4)$ as

$$
V=\Delta^{(4)} V^{(-4)}, \quad \mathbb{D} V^{(-4)}=-2 V^{(-4)}, \quad S_{\hat{\alpha}}^{i} V^{(-4)}=0 .
$$

The vector multiplet field strength (6.37) then takes the form

$$
W=-\frac{\mathrm{i}}{16 \pi} \oint(v, \mathrm{~d} v) \nabla^{(-2)} \Delta^{(4)} V^{(-4)}(v) .
$$

Making use of the identity

$$
\nabla^{(-2)} \Delta^{(4)}=\frac{3}{5} v_{i} v_{j} \nabla_{k l} \Delta^{i j k l},
$$

which follows from (5.23), we can perform the contour integral and obtain the following alternative expression for the field strength

$$
W=-\frac{3 \mathrm{i}}{5} \oint \frac{(v, \mathrm{~d} v)}{16 \pi} v_{k} v_{l} \nabla_{i j} \Delta^{i j k l} V^{(-4)}(v)=-\frac{3 \mathrm{i}}{40} \nabla_{i j} \Delta^{i j k l} V_{k l} .
$$

Here we have defined the superfield $V_{i j}$ as

$$
V_{i j}:=\oint \frac{(v, \mathrm{~d} v)}{2 \pi} v_{i} v_{j} V^{(-4)}(v) .
$$

By construction $V_{i j}$ is a real primary superfield of dimension $-2, \mathbb{D} V_{i j}=-2 V_{i j}$. It is also possible to prove that, due to $(5.4 \mathrm{~b})$ and the definition (6.49), $V_{i j}$ correctly transforms as an isovector under $\mathrm{SU}(2)$ transformations. Note that $V_{i j}$ is the analogue of Mezincescu's prepotential [67] (see also [68] and [69]) for the $4 \mathrm{D} \mathcal{N}=2$ abelian vector multiplet. To conclude, we note that $V_{i j}$ is defined up to gauge transformations of the form

$$
\delta V_{k l}=\nabla_{\hat{\alpha}}^{p} \Lambda_{k l p}^{\hat{\alpha}}, \quad \Lambda_{k l p}^{\hat{\alpha}}=\Lambda_{(k l p)}^{\hat{\alpha}},
$$

with the gauge parameter being $\Lambda^{\hat{\alpha}_{i j k}}$ a primary superfield,

$$
S_{\hat{\alpha}}^{i} \Lambda^{\hat{\beta}}{ }_{j k l}=0, \quad \mathbb{D} \Lambda^{\hat{\beta}}{ }_{j k l}=-\frac{5}{2} \Lambda^{\hat{\beta}}{ }_{j k l} .
$$

The gauge invariance follows from the fact that $\nabla_{i j} \Delta^{i j k l} \nabla_{\hat{\alpha}}^{p} \Lambda^{\hat{\alpha}}{ }_{k l p}=0$, as can be proven using (5.23).

\subsection{Composite $\mathcal{O}(2)$ multiplet}

Consider a locally supersymmetric theory that involves an abelian vector multiplet as one of the dynamical multiplets. Let $S[V(v)]=S\left[V_{i j}\right]$ be the corresponding gauge invariant 
action. A variation of the action with respect to the vector multiplet may be represented in two different forms,

$$
\begin{aligned}
\delta S & =\frac{1}{2 \pi} \oint(v, \mathrm{~d} v) \int \mathrm{d}^{5 \mid 8} z E C^{(-4)} \mathbb{H}^{(2)} \delta V \\
& =\int \mathrm{d}^{5 \mid 8} z E \mathbb{H}^{i j} \delta V_{i j},
\end{aligned}
$$

for some real weight-2 tropical multiplet $\mathbb{H}^{(2)}(v)$,

$$
\nabla_{\hat{\alpha}}^{(1)} \mathbb{H}^{(2)}=0,
$$

and some real isovector $\mathbb{H}^{i j}=\mathbb{H}^{j i}$, which are primary superfields of dimension +3 . The theory under consideration may also involve hypermultiplets charged under the $\mathrm{U}(1)$ gauge group. We assume that these hypermultiplets obey the corresponding equations of motion. Then the above variation vanishes when $\delta V$ or $\delta V^{i j}$ is a gauge transformation. This property has two different, but equivalent, manifestations. Firstly, the variation (6.52a) is equal to zero for the gauge transformation (6.44), hence

$$
\oint(v, \mathrm{~d} v) \int \mathrm{d}^{5 \mid 8} z E C^{(-4)} \mathbb{H}^{(2)} \lambda=0
$$

for an arbitrary weight-0 arctic multiplet $\lambda(v)$. This implies that $\mathbb{H}^{(2)}(v)$ is an $\mathcal{O}(2)$ multiplet,

$$
\mathbb{H}^{(2)}(v)=\mathbb{H}^{i j} v_{i} v_{j}
$$

Secondly, the variation (6.52b) is equal to zero for the gauge transformation (6.50). This means

$$
\int \mathrm{d}^{5 \mid 8} z E \Lambda_{i j k}^{\hat{\alpha}} \nabla_{\hat{\alpha}}^{i} \mathbb{H}^{j k}=0,
$$

and hence

$$
\nabla_{\hat{\alpha}}^{(i} \mathbb{H}^{j k)}=0
$$

The superfields $\mathbb{H}^{(2)}(v)$ and $\mathbb{H}^{i j}$ defined by eqs. (6.52a) and (6.52b), respectively, are related to each other according to (6.55), as follows from (6.49).

In summary, any gauge theory of the abelian vector multiplet possesses a composite $\mathcal{O}(2)$ multiplet, $\mathbb{H}^{i j}$. The equation of motion for the vector multiplet is $\mathbb{H}^{i j}=0$.

\section{The $\mathcal{O}(2)$ multiplet in conformal superspace}

In the previous section we gave the prepotential description of the Yang-Mills multiplet. Here we develop a prepotential formulation for the $\mathcal{O}(2)$ multiplet, a dual version of the hypermultiplet. In the $4 \mathrm{D} \mathcal{N}=2$ case, it is known that the $\mathcal{O}(2)$ multiplet constraints

$$
\nabla_{\alpha}^{(i} G^{j k)}=0, \quad \bar{\nabla}_{\dot{\alpha}}^{(i} G^{j k)}=0,
$$

may be solved in conformal superspace in terms of a complex primary scalar $U$ of dimension $-1, \mathbb{D} U=-U$, as

$$
G^{i j}=\frac{1}{192}\left(\nabla^{i j} \bar{\nabla}^{k l} \bar{\nabla}_{k l} \bar{U}+\bar{\nabla}^{i j} \nabla^{k l} \nabla_{k l} U\right),
$$


see, e.g., [69] for a detailed discussion. As will be demonstrated below, an analogous six-derivative representation for the $\mathcal{O}(2)$ multiplet exists in five dimensions, but the corresponding prepotential is a real dimensionless scalar.

\subsection{Prepotential formulation for the $\mathcal{O}(2)$ multiplet}

In five dimensions, the $\mathcal{O}(2)$ multiplet $G^{i j}=G^{j i}$ is characterized by the properties

$$
\nabla_{\hat{\alpha}}^{(i} G^{j k)}=0, \quad S_{\hat{\alpha}}^{i} G^{j k}=0, \quad \mathbb{D} G^{j k}=3 G^{j k} .
$$

We always assume $G^{i j}$ to be real, $\overline{G^{i j}}=G_{i j}=\varepsilon_{i k} \varepsilon_{j l} G^{k l}$. It turns out that the constraints (7.3) may be solved in terms of a primary real dimensionless scalar $\Omega$,

$$
S_{\hat{\alpha}}^{i} \Omega=0, \quad \mathbb{D} \Omega=0,
$$

and the solution is

$$
G^{i j}=-\frac{3 \mathrm{i}}{40} \Delta^{i j k l} \nabla_{k l} \Omega
$$

or, equivalently,

$$
G^{(2)}=v_{i} v_{j} G^{i j}=-\frac{\mathrm{i}}{8} \Delta^{(4)} \nabla^{(-2)} \Omega .
$$

Note that representation (7.5a) follows from (7.5b) by applying (5.23).

In appendix $\mathrm{E}$ we prove that the decomposition (7.5) is the most general solution to (7.3) in the flat case by making use of the harmonic superspace techniques [58, 59]. Here we demonstrate that (7.5) defines a primary $\mathcal{O}(2)$ superfield in conformal superspace.

It follows from $(7.5 \mathrm{~b})$ that $G^{(2)}$ is analytic, $\nabla_{\hat{\alpha}}^{(1)} G^{(2)}=0$. It is also obvious that $G^{(2)}$ has the right dimension, $\mathbb{D} G^{(2)}=3$, since $\Omega$ is dimensionless. It is slightly more involved to check that $S_{\hat{\alpha}}^{i} G^{(2)}=0$, which is equivalent to proving the two conditions $S_{\hat{\alpha}}^{(1)} G^{(2)}=0$ and $S_{\hat{\alpha}}^{(-1)} G^{(2)}=0$.

Let us first consider

$$
S_{\hat{\alpha}}^{(-1)} G^{(2)}=-\frac{\mathrm{i}}{8}\left[S_{\hat{\alpha}}^{(-1)}, \Delta^{(4)}\right] \nabla^{(-2)} \Omega-\frac{\mathrm{i}}{8} \Delta^{(4)}\left[S_{\hat{\alpha}}^{(-1)}, \nabla^{(-2)}\right] \Omega .
$$

It is straightforward to check that the second term on the right is identically zero:

$$
\begin{aligned}
{\left[S_{\hat{\alpha}}^{(-1)}, \nabla^{(-2)}\right] \Omega } & =\left(\left\{S_{\hat{\alpha}}^{(-1)}, \nabla^{\hat{\beta}(-1)}\right\} \nabla_{\hat{\beta}}^{(-1)}-\nabla^{\hat{\beta}(-1)}\left\{S_{\hat{\alpha}}^{(-1)}, \nabla_{\hat{\beta}}^{(-1)}\right\}\right) \Omega \\
& =6\left(J^{(-2)} \nabla_{\hat{\alpha}}^{(-1)}+\nabla_{\hat{\alpha}}^{(-1)} J^{(-2)}\right) \Omega=0,
\end{aligned}
$$

as a consequence of

$$
\left[J^{(-2)}, \nabla_{\hat{\alpha}}^{(-1)}\right]=0, \quad J^{(-2)}:=\frac{1}{(v, u)^{2}} u_{i} u_{j} J^{i j}
$$

It remains to show that $\left[S_{\hat{\alpha}}^{(-1)}, \Delta^{(4)}\right] \nabla^{(-2)} \Omega=0$. Using (5.30b) we obtain

$$
\left[S_{\hat{\alpha}}^{(-1)}, \Delta^{(4)}\right] \nabla^{(-2)} \Omega=\frac{1}{24} \varepsilon^{\hat{\beta} \hat{\gamma} \hat{\delta} \hat{\rho}} \varepsilon_{\hat{\alpha} \hat{\beta}} \nabla_{\hat{\gamma}}^{(1)} \nabla_{\hat{\delta}}^{(1)} \nabla_{\hat{\rho}}^{(1)}\left(8-2 \mathbb{D}-6 J^{(0)}\right) \nabla^{(-2)} \Omega \equiv 0 .
$$


Since $\partial^{(2)} G^{(2)}=0$, we also find $S_{\hat{\alpha}}^{(1)} G^{(2)}=\partial^{(2)} S_{\hat{\alpha}}^{(-1)} G^{(2)}=0$. Thus we have shown that the superfield $G^{(2)}$ defined by (7.5) is primary.

A crucial property of the superfield $G^{(2)}$ defined by (7.5) is that it is invariant under gauge transformations of $\Omega$ of the form

$$
\delta \Omega=-\frac{\mathrm{i}}{2}\left(\Gamma^{\hat{a}}\right)^{\hat{\alpha} \hat{\beta}} \nabla_{\hat{\alpha}}^{i} \nabla_{\hat{\beta}}^{j} B_{\hat{a} i j},
$$

where the gauge parameter is assumed to have the properties

$$
B_{\hat{a}}^{i j}=B_{\hat{a}}^{j i}, \quad S_{\hat{\alpha}}^{i} B_{\hat{a}}^{j k}=0, \quad \mathbb{D} B_{\hat{a}}^{i j}=-B_{\hat{a}}^{i j}
$$

and is otherwise arbitrary. It is an instructive exercise to show that the variation $\delta \Omega$ defined by (7.10) and (7.11) is a primary dimensionless superfield. Appendix F is devoted to the proof that the transformation (7.10) leaves invariant the field strength $G^{(2)}$ defined by (7.5).

\subsection{Composite vector multiplet}

Consider a dynamical system involving an $\mathcal{O}(2)$ multiplet $G^{i j}$ as one of the dynamical multiplets. The action may be viewed as a functional of the field strength, $S\left[G^{i j}\right]$, or as a gauge invariant functional, $S[\Omega]$, of the prepotential $\Omega$. Giving the prepotential an infinitesimal displacement changes the action as follows:

$$
\delta S=\int \mathrm{d}^{5 \mid 8} z E \mathbb{W} \delta \Omega
$$

for some real scalar $\mathbb{W}$, which is a primary superfield of dimension +1 . The variation must vanish if $\delta \Omega$ is a gauge transformation of the form (7.10). This holds if $\mathbb{W}$ obeys the equation

$$
\nabla_{\hat{\alpha}}^{(i} \nabla_{\hat{\beta}}^{j)} \mathbb{W}=\frac{1}{4} \varepsilon_{\hat{\alpha} \hat{\beta}} \nabla^{\hat{\gamma}(i} \nabla_{\hat{\gamma}}^{j)} \mathbb{W}
$$

which is the Bianchi identity for the field strength of an abelian vector multiplet, see eq. (2.49).

In summary, any dynamical system involving an $\mathcal{O}(2)$ multiplet $G^{i j}$ possesses a composite vector multiplet, $\mathbb{W}$. The equation of motion for the $\mathcal{O}(2)$ multiplet is $\mathbb{W}=0$.

\subsection{BF coupling}

Consider the following Lagrangian

$$
\mathcal{L}_{\mathrm{BF}}^{(2)}=V G^{(2)}
$$

that describes a $\mathrm{BF}$ coupling of a vector multiplet and an $\mathcal{O}(2)$ multiplet. The action principle (5.35) with $\mathcal{L}_{\mathrm{BF}}^{(2)}=V G^{(2)}$ will be referred to as the $\mathrm{BF}$ action.

The BF action involves the tropical prepotential of the vector multiplet, $V\left(v^{i}\right)$, and the field strength of the $\mathcal{O}(2)$ multiplet, $G^{(2)}$. It can be rewritten in a different form involving 
the field strength of the vector multiplet, $W$, and the prepotential of the $\mathcal{O}(2)$ multiplet, $\Omega$. This is achieved by expressing $G^{(2)}$ in terms of $\Omega$ and then integrating by parts to obtain

$$
\begin{aligned}
S\left[\mathcal{L}_{\mathrm{BF}}^{(2)}\right] & =\oint \frac{(v, \mathrm{~d} v)}{16 \pi \mathrm{i}} \int \mathrm{d}^{5 \mid 8} z E C^{(-4)} V \Delta^{(+4)} \nabla^{(-2)} \Omega \\
& =\int \mathrm{d}^{5 \mid 8} z E \Omega W .
\end{aligned}
$$

By using (7.5) and (6.48) together with integration by parts, the action may be rewritten in another equivalent form that involves Mezincescu's prepotential $V_{i j}$ and the field strength $G^{i j}$. One obtains

$$
S\left[\mathcal{L}_{\mathrm{BF}}^{(2)}\right]=\int \mathrm{d}^{5 \mid 8} z E \Omega W=\int \mathrm{d}^{5 \mid 8} z E G^{i j} V_{i j} .
$$

One may prove that the functionals $\int \mathrm{d}^{5 \mid 8} z E \Omega W$ and $\int \mathrm{d}^{5 \mid 8} z E G^{i j} V_{i j}$ are invariant under the gauge transformations (7.10) and (6.50), respectively.

\subsection{Gauge invariance}

The results of the previous subsection allow us to prove the important relation (5.45a). For this we choose $V=\lambda+\breve{\lambda}$ in the BF Lagrangian (7.14), where $\lambda(v)$ is a weight- 0 arctic multiplet. Since the tropical prepotential is pure gauge, the field strength vanishes, $W=0$. Then eq. (7.16) leads to $S\left[(\lambda+\breve{\lambda}) G^{(2)}\right]=0$, which is the required result (5.45a). Since $\lambda$ is complex, we can replace $\lambda(v) \rightarrow \mathrm{i} \lambda(v)$ and obtain $S\left[\mathrm{i}(\lambda-\breve{\lambda}) G^{(2)}\right]=0$. These two relations lead to $(5.45 \mathrm{~b})$, and thus

$$
\oint(v, \mathrm{~d} v) \int \mathrm{d}^{5 \mid 8} z E C^{(-4)} G^{(2)} \lambda=0,
$$

where $G^{(2)}(v)$ is an $\mathcal{O}(2)$ multiplet and $\lambda(v)$ is a weight- 0 arctic multiplet.

\subsection{Universality of the BF action}

The goal of this subsection is to demonstrate that the supersymmetric action (5.35) can be rewritten as a $\mathrm{BF}$ action under the assumption that a special vector multiplet exists.

Consider the action (5.35) written as (5.39) with $\mathcal{U}^{(-2)}$ a prepotential for the Lagrangian $\mathcal{L}^{(2)}$. Now let $W$ be the field strength of a compensating vector multiplet. We insert the unity $1=W / W$ in the right hand side of (5.39) and represent $W$ in the numerator according to (6.37). After that we change the order of the contour integrals and integrate $\nabla^{(-2)}$ by parts. Finally, we insert the unity $1=\Delta^{(4)} C^{(-4)}$ and integrate by parts. The final result is

$$
S=\frac{1}{2 \pi} \oint_{\gamma}(v, \mathrm{~d} v) \int \mathrm{d}^{5 \mid 8} z E C^{(-4)} V \mathbb{G}^{(2)},
$$

where $V$ is the tropical prepotential for the vector multiplet and the composite superfield $\mathbb{G}^{(2)}$ is defined by

$$
\begin{aligned}
\mathbb{G}^{(2)} & =-\frac{\mathrm{i}}{8} \Delta^{(4)} \nabla^{(-2)} \Omega, \\
\Omega & :=\frac{1}{2 \pi W} \oint_{\gamma}(v, \mathrm{~d} v) \mathcal{U}^{(-2)} .
\end{aligned}
$$


According to (7.5), the superfield $\mathbb{G}^{(2)}$ is an $\mathcal{O}(2)$ multiplet. Note that it is possible to give some alternative expressions for $\Omega$ in $(7.19 \mathrm{~b})$. Consider a weight -4 isotwistor superfield $\tilde{C}^{(-4)}$ such that $\Delta^{(4)} \tilde{C}^{(-4)}=1$. This does not necessarily have to be equal to $C^{(-4)}$. Given $\tilde{C}^{(-4)}$, the superfield

$$
\mathcal{U}^{(-2)}:=\tilde{C}^{(-4)} \mathcal{L}^{(2)}
$$

is a prepotential for the projective Lagrangian $\mathcal{L}^{(2)}$. Hence we have the equivalent expression

$$
\Omega:=\frac{1}{2 \pi W} \oint_{\gamma}(v, \mathrm{~d} v) \tilde{C}^{(-4)} \mathcal{L}^{(2)}
$$

Note that in the presence of the vector multiplet compensator a natural choice for $\tilde{C}^{(-4)}$ is given by (5.42). Then we find

$$
\Omega:=\frac{2 W^{3}}{3 \pi} \oint_{\gamma}(v, \mathrm{~d} v) \frac{\mathcal{L}^{(2)}}{\left(H_{\mathrm{VM}}^{(2)}\right)^{2}} .
$$

\subsection{Full superspace invariants}

Consider an invariant that can be represented as an integral over the full superspace $\mathcal{M}^{5 \mid 8}$,

$$
S[\mathcal{L}]=\int \mathrm{d}^{5 \mid 8} z E \mathcal{L}
$$

where $\mathcal{L}$ is a conformal primary superfield of dimension $+1, \mathbb{D} \mathcal{L}=\mathcal{L}$. This invariant may be represented in the form $(5.35)$, in which $\mathcal{L}^{(2)}$ reads

$$
\mathcal{L}^{(2)}=-\frac{2}{G^{(2)}} \Delta^{(4)}(G \mathcal{L}) .
$$

Here $\Delta^{(4)}$ is the covariant analytic projection operator (5.22) and $G^{(2)}=v_{i} v_{j} G^{i j}$ is an $\mathcal{O}(2)$ multiplet such that

$$
G^{2}:=\frac{1}{2} G^{i j} G_{i j}
$$

is nowhere vanishing, $G \neq 0$. The Lagrangian (7.24) is an example of a covariant rational projective multiplet ${ }^{18}$ in the sense that it has the structure $H^{(4)} / G^{(2)}$, for some $\mathcal{O}(4)$ multiplet $H^{(4)}(v)$.

\subsection{Prepotentials for $\mathcal{O}(4+n)$ multiplets}

Let $H^{(4)}(v)$ be an $\mathcal{O}(4)$ multiplet. It may be shown that

$$
H^{(4)}(v)=\Delta^{(4)} \Phi, \quad S_{\hat{\alpha}}^{i} \Phi=0, \quad \mathbb{D} \Phi=4 \Phi,
$$

for some primary scalar prepotential $\Phi$, see appendix G. The $\mathcal{O}(4)$ multiplet in (7.24), $\Delta^{(4)}(G \mathcal{L})$, is a special case of this result.

More generally, let $H^{(4+n)}(v)$ be an $\mathcal{O}(4+n)$ multiplet, with $n=0,1, \ldots$ It may be represented in the form

$$
H^{(4+n)}(v)=\Delta^{(4)} \Phi^{(n)}, \quad \Phi^{(n)}(v)=\Phi^{i_{1} \ldots i_{n}} v_{i_{1}} \ldots v_{i_{n}},
$$

for some primary superfield $\Phi^{i_{1} \ldots i_{n}}$ of dimension $\left(4+\frac{3}{2} n\right)$, see appendix G.

\footnotetext{
${ }^{18}$ In the $4 \mathrm{D} \mathcal{N}=2$ super Poincaré case, rational projective multiplets were first introduced by Lindström and Roček [36].
} 


\section{Superform formulation for the BF action}

In section 7 we demonstrated the universality of the BF action

$$
S_{\mathrm{BF}}=\frac{1}{2 \pi} \oint_{\gamma}(v, \mathrm{~d} v) \int \mathrm{d}^{5 \mid 8} z E C^{(-4)} \mathcal{L}_{\mathrm{BF}}^{(2)}, \quad \mathcal{L}_{\mathrm{BF}}^{(2)}=V G^{(2)} .
$$

The component structure of $S_{\mathrm{BF}}$ is of primary importance for applications. For the analogous action in $4 \mathrm{D} \mathcal{N}=2$ supergravity, two procedures have been developed to reduce the action to components. One of them [55] directly carries out the integration over the Grassmann variables in the action. The other approach [70] provides a superform construction for the action ${ }^{19}$ which immediately leads to the component action. The latter has turned out to be fruitful for various generalizations, such as the $\mathcal{N}$-extended conformal supergravity actions $[48,49,75]$ and the Chern-Simons actions [76] in three dimensions and the non-abelian Chern-Simons action in 5D $\mathcal{N}=1$ Minkowski superspace [77]. Here we apply the ideas put forward in [70] to derive a superform formulation for the action $S_{\mathrm{BF}}$.

\subsection{Superform geometry of the $\mathcal{O}(2)$ multiplet}

The $\mathcal{O}(2)$ multiplet can be described by a three-form gauge potential $\mathcal{B}=\frac{1}{3 !} E^{\hat{C}} \wedge E^{\hat{B}} \wedge$ $E^{\hat{A}} \mathcal{B}_{\hat{A} \hat{B} \hat{C}}$ possessing the gauge transformation

$$
\delta \mathcal{B}=\mathrm{d} \rho,
$$

where $\rho$ is a 2 -form gauge parameter. The corresponding field strength is

$$
\Phi=\mathrm{d} \mathcal{B}=\frac{1}{4 !} E^{\hat{D}} \wedge E^{\hat{C}} \wedge E^{\hat{B}} \wedge E^{\hat{A}} \Phi_{\hat{A} \hat{B} \hat{C} \hat{D}}
$$

where

$$
\Phi_{\hat{A} \hat{B} \hat{C} \hat{D}}=4 \nabla_{[\hat{A}} \mathcal{B}_{\hat{B} \hat{C} \hat{D}\}}+6 \mathscr{T}_{[\hat{A} \hat{B}} \hat{E}_{|\hat{E}| \hat{C} \hat{D}\}} .
$$

The field strength must satisfy the Bianchi identity

$$
\nabla_{[\hat{A}} \Phi_{\hat{B} \hat{C} \hat{D} \hat{E}\}}+2 \mathscr{T}_{[\hat{A} \hat{B}}{ }^{\hat{F}} \Phi_{|\hat{F}| \hat{C} \hat{D} \hat{E}\}}=0
$$

In order to describe the $\mathcal{O}(2)$ multiplet we need to impose some covariant constraints on the field strength $\Phi$. We choose the constraints

$$
\Phi_{\hat{\alpha} \hat{\beta} \hat{\gamma} \hat{\delta}}^{i j k l}=\Phi_{\hat{a} \hat{\beta} \hat{\gamma} \hat{\delta}}^{j k l}=0, \quad \Phi_{\hat{a} \hat{b} \hat{\alpha} \hat{\beta}}^{i j}=8 \mathrm{i}\left(\sum_{\hat{a} \hat{b}}\right)_{\hat{\alpha} \hat{\beta}} G^{i j}
$$

where $G^{i j}=G^{j i}$ is a dimension-3 primary superfield. The constraints allow one to solve for the remaining components of $\Phi$ in terms of $G^{i j}$. The solution is

$$
\begin{aligned}
& \Phi_{\hat{a} \hat{b} \hat{c} \hat{\alpha}}^{i}=-\frac{2}{3} \varepsilon_{\hat{a} \hat{b} \hat{c} \hat{d} \hat{e}}\left(\Sigma^{\hat{d} \hat{e}}\right)_{\hat{\alpha}}^{\hat{\beta}} \nabla_{\hat{\beta} j} G^{j i}=-2 \varepsilon_{\hat{a} \hat{b} \hat{c} \hat{d} \hat{e}}\left(\Sigma^{\hat{d} \hat{e}}\right)_{\hat{\alpha}}^{\hat{\beta}} \varphi_{\hat{\beta}}^{i}, \\
& \Phi_{\hat{a} \hat{b} \hat{c} \hat{d}}=\frac{i}{12} \varepsilon_{\hat{a} \hat{b} \hat{c} \hat{d} \hat{e}}\left(\Gamma^{\hat{e}}\right)^{\hat{\alpha} \hat{\beta}} \nabla_{\hat{\alpha}}^{i} \nabla_{\hat{\beta}}^{j} G_{i j} \equiv \varepsilon_{\hat{a} \hat{b} \hat{c} \hat{d} \hat{e}} \Phi^{\hat{e}},
\end{aligned}
$$

\footnotetext{
${ }^{19}$ This approach makes use of the superform formalism to construct supersymmetric invariants [71-74].
} 
where $G^{i j}$ satisfies the constraint for the $\mathcal{O}(2)$ multiplet

$$
\nabla_{\hat{\alpha}}^{(i} G^{j k)}=0
$$

and we have introduced the superfields

$$
\begin{aligned}
\varphi_{\hat{\alpha}}^{i} & :=\frac{1}{3} \nabla_{\hat{\alpha} j} G^{i j}, \\
F & :=\frac{\mathrm{i}}{12} \nabla^{\hat{\gamma} i} \nabla_{\hat{\gamma}}^{j} G_{i j}=-\frac{\mathrm{i}}{4} \nabla^{\hat{\gamma} k} \varphi_{\hat{\gamma} k} .
\end{aligned}
$$

The Bianchi identities also imply the differential condition on $\Phi_{\hat{a}}$

$$
\nabla^{\hat{a}} \Phi_{\hat{a}}+5 \mathrm{i} X^{\hat{\gamma} k} \varphi_{\hat{\gamma} k}=\hat{\nabla}^{\hat{a}} \Phi_{\hat{a}}=0 .
$$

\subsection{Superform action for the $\mathcal{O}(2)$ multiplet}

The superform formulation in the previous subsection gives a geometric description for the $\mathcal{O}(2)$ multiplet. As we will see, it is a useful ingredient in the construction of the BF action principle. Below we describe the general setup, the construction of the superform action and its corresponding component action.

\subsubsection{General setup}

The superform approach to constructing supersymmetric invariants [71-74] is based on the use of a closed superform. In five-dimensional spacetime $\mathcal{M}^{5}$, which is the body of the $\mathcal{N}=1$ curved superspace $\mathcal{M}^{5 \mid 8}$, the formalism requires the use of a closed five-form

$$
\mathfrak{J}=\frac{1}{5 !} E^{\hat{E}} \wedge E^{\hat{D}} \wedge E^{\hat{C}} \wedge E^{\hat{B}} \wedge E^{\hat{A}} \mathfrak{J}_{\hat{A} \hat{B} \hat{C} \hat{D} \hat{E}}, \quad \mathrm{~d} \mathfrak{J}=0 .
$$

Given such a superform, one can construct the supersymmetric invariant

$$
S=\int_{\mathcal{M}^{5}} i^{*} \mathfrak{J}
$$

where $i: \mathcal{M}^{5} \rightarrow \mathcal{M}^{5 \mid 8}$ is the inclusion map. Invariance under arbitrary general coordinate transformations of the superspace follows from the transformation of $\mathfrak{J}$,

$$
\delta_{\xi} \mathfrak{J}=\mathcal{L}_{\xi} \mathfrak{J} \equiv i_{\xi} \mathrm{d} \mathfrak{J}+\mathrm{d} i_{\xi} \mathfrak{J}=\mathrm{d} i_{\xi} \mathfrak{J} .
$$

The closed form $\mathfrak{J}$ is required to transform as an exact form under all gauge symmetries,

$$
\mathrm{d} \mathfrak{J}=\mathrm{d} \Theta
$$

which ensures eq. (8.11) is a suitable candidate for an action. In conformal supergravity, suitable actions must be invariant under the standard superconformal transformations. This requires that $\mathfrak{J}$ transforms by an exact form under the standard superconformal transformations,

$$
\delta_{\mathcal{H}} \mathfrak{J}=\mathrm{d} \Theta\left(\Lambda^{\underline{a}}\right), \quad \Lambda=\Lambda^{\underline{a}} X_{\underline{a}} .
$$


Locally superconformal matter actions are usually associated with closed five-forms that are invariant,

$$
\delta_{\mathcal{H}} \mathfrak{J}=0
$$

This is equivalent to the condition

$$
X_{\underline{a}} \mathfrak{J}_{\hat{A}_{1} \cdots \hat{A}_{p}}=-f_{\underline{a}\left[\hat{A}_{1}\right.} \hat{D}_{\mathfrak{J}_{\left.|\hat{D}| \hat{A}_{2} \cdots \hat{A}_{p}\right\}}} .
$$

The $S$-invariance, $S_{\hat{\alpha} i} \mathfrak{J}=0$, is non-trivial and we will call a superform that is $S$-invariant a primary superform. ${ }^{20}$ In general, a primary $p$-form $\Sigma$ satisfies

$$
S_{\hat{\alpha} i} \Sigma_{\hat{A}_{1} \cdots \hat{A}_{p}}=\mathrm{i} p\left(\Gamma_{\hat{a}}\right)_{\hat{\alpha}}{ }^{\hat{\beta}} \Sigma_{\hat{\beta} i\left[\hat{A}_{2} \cdots \hat{A}_{p}\right.} \delta_{\left.\hat{A}_{1}\right\}}^{\hat{a}},
$$

which implies the condition

$$
S_{\hat{\beta} j} \Sigma_{\hat{a}_{1} \cdots \hat{a}_{n}}{ }_{\hat{\alpha}_{1}}^{i_{1}} \ldots{ }_{\hat{\alpha}_{p-n}}^{i_{p-n}}=\mathrm{i} n\left(\Gamma_{\left[\hat{a}_{1}\right.}\right)_{\hat{\beta}}{ }_{\hat{\gamma}}^{\hat{\gamma}} \sum_{\left.\hat{\gamma} j \hat{a}_{2} \cdots \hat{a}_{n}\right]} i_{\hat{\alpha}_{1}}^{i_{1}} \ldots{ }_{\hat{\alpha}_{p-n}}^{i_{p-n}} .
$$

\subsubsection{Superform action for the $\mathcal{O}(2)$ multiplet}

In order to construct a closed form for the action we will first consider the superform equation

$$
\mathrm{d} \Sigma=F \wedge \Phi,
$$

where $\Sigma$ is some five-form and $F$ is the field strength for an abelian vector multiplet with gauge one-form $V$ and field strength $W$ (see section 2.6).

It turns out there exist two solutions to eq. (8.19) that do not differ by an exact form. The first solution is

$$
\Sigma_{V}=V \wedge \Phi .
$$

The second solution is the result of the constraints that have been imposed on the components of $F$ and $\Phi$. If we assume that this solution is primary then we may write the Bianchi identity in terms of the covariant derivatives as follows:

$$
\nabla_{[\hat{A}} \Sigma_{\hat{B} \hat{C} \hat{D} \hat{E} \hat{F}\}}+\frac{5}{2} \mathscr{T}_{[\hat{A} \hat{B}} \hat{G}^{\Sigma_{|\hat{G}| \hat{C} \hat{D} \hat{E} \hat{F}\}}}=\frac{5}{2} F_{[\hat{A} \hat{B}} \Phi_{\hat{C} \hat{D} \hat{E} \hat{F}\}}
$$

Making use of the components of $F$ and $\Phi$, one finds the solution:

$$
\begin{aligned}
\Sigma_{\hat{a} \hat{b} \hat{c} \hat{\alpha} \hat{\beta}}^{i j} & =-4 \mathrm{i} \varepsilon_{\hat{a} \hat{b} \hat{c} \hat{d} \hat{e}}\left(\Sigma^{\hat{d} \hat{e}}\right)_{\hat{\alpha} \hat{\beta}} W G^{i j}, \\
\Sigma_{\hat{a} \hat{b} \hat{c} \hat{d} \hat{\alpha}}^{i} & =2 \varepsilon_{\hat{a} \hat{b} \hat{c} \hat{d} \hat{e}}\left(\Gamma^{\hat{e}}\right)_{\hat{\alpha}}^{\hat{\beta}}\left(W \varphi_{\hat{\beta}}^{i}+\mathrm{i} \lambda_{\hat{\beta} j} G^{j i}\right), \\
\Sigma_{\hat{a} \hat{b} \hat{c} \hat{d} \hat{e}} & =-\varepsilon_{\hat{a} \hat{b} \hat{c} \hat{d} \hat{e}}\left(W F+X^{i j} G_{i j}+2 \lambda^{\hat{\gamma} k} \varphi_{\hat{\gamma} k}\right)
\end{aligned}
$$

with the remaining components vanishing. Here we have made use of the following useful identities:

$$
\begin{aligned}
\nabla_{\hat{\alpha}}^{i} G^{j k} & =2 \varepsilon^{i(j} \varphi_{\hat{\alpha}}^{k)}, \\
\nabla_{\hat{\alpha}}^{i} \varphi_{\hat{\beta}}^{j} & =-\frac{\mathrm{i}}{2} \varepsilon^{i j} \varepsilon_{\hat{\alpha} \hat{\beta}} F+\frac{\mathrm{i}}{2} \varepsilon^{i j} \Phi_{\hat{\alpha} \hat{\beta}}+\mathrm{i} \nabla_{\hat{\alpha} \hat{\beta}} G^{i j},
\end{aligned}
$$

\footnotetext{
${ }^{20} S$-invariance automatically implies $K$-invariance.
} 


$$
\begin{aligned}
\nabla_{\hat{\alpha}}^{i} F & =-2 \nabla_{\hat{\alpha}}^{\hat{\beta}} \varphi_{\hat{\beta}}^{i}-6 W_{\hat{\alpha} \hat{\beta}} \varphi^{\hat{\beta} i}-9 X_{\hat{\alpha} j} G^{i j} \\
\nabla_{\hat{\alpha}}^{i} \Phi_{\hat{a}} & =4\left(\Sigma_{\hat{a} \hat{b}}\right)_{\hat{\alpha}}^{\hat{\beta}} \nabla^{\hat{b}} \varphi_{\hat{\beta}}^{i}-4\left(\Gamma_{\hat{a}}\right)_{\hat{\alpha}}^{\hat{\beta}} W_{\hat{\beta} \hat{\gamma}} \varphi^{\hat{\gamma} i}-6\left(\Gamma_{\hat{a}}\right)_{\hat{\alpha}}^{\hat{\beta}} X_{\hat{\beta} j} G^{i j}
\end{aligned}
$$

The second solution is expressed entirely in terms of $G^{i j}$ and its covariant derivatives and we will denote it by $\Sigma_{G}$. Making use of the following identities

$$
\begin{aligned}
S_{\hat{\alpha}}^{i} \varphi_{\hat{\beta}}^{j} & =-6 \varepsilon_{\hat{\alpha} \hat{\beta}} G^{i j}, \\
S_{\hat{\alpha}}^{i} F & =6 \mathrm{i} \varphi_{\hat{\alpha}}^{i}, \\
S_{\hat{\alpha}}^{i} \Phi_{\hat{b}} & =-8 \mathrm{i}\left(\Gamma_{\hat{b}}\right)_{\hat{\alpha}} \hat{\beta} \varphi_{\hat{\beta}}^{i},
\end{aligned}
$$

one can check that $\Sigma_{G}$ is primary, i.e. it satisfies eq. (8.18). Similarly one can show $\Phi$ is primary and hence $\Sigma_{V}$ is primary also.

It is now straightforward to construct a closed invariant five-form. One may simply take the difference between $\Sigma_{V}$ and $\Sigma_{G}$,

$$
\mathfrak{J}=\Sigma_{V}-\Sigma_{G}=V \wedge \Phi-\Sigma_{G}
$$

\subsubsection{Component BF action}

Having derived $\mathfrak{J}$ we can now make use of the action principle (8.10) to construct the corresponding component action. The Lagrangian is given by ${ }^{21}$

$$
e^{-1 *} \mathfrak{J}=\frac{1}{5 !} \varepsilon^{\hat{m} \hat{n} \hat{p} \hat{q} \hat{r}} \mathfrak{J}_{\hat{m} \hat{n} \hat{p} \hat{q} \hat{r}}=\frac{1}{4 !} \varepsilon^{\hat{m} \hat{n} \hat{p} \hat{q} \hat{r}} V_{\hat{m}} \Phi_{\hat{n} \hat{p} \hat{q} \hat{r}}-\frac{1}{5 !} \varepsilon^{\hat{m} \hat{n} \hat{p} \hat{q} \hat{r}} \sum_{\hat{m} \hat{n} \hat{p} \hat{q} \hat{r}}
$$

where

$$
\begin{aligned}
& \frac{1}{5 !} \varepsilon^{\hat{m} \hat{n} \hat{p} \hat{q} \hat{r}} \Sigma_{\hat{m} \hat{n} \hat{p} \hat{q} \hat{r}}\left|=\frac{1}{5 !} \varepsilon^{\hat{m} \hat{n} \hat{p} \hat{q} \hat{r}} E_{\hat{r}}^{\hat{E}} E_{\hat{q}}{ }^{\hat{D}} E_{\hat{p}} \hat{C} E_{\hat{n}}{ }^{\hat{B}} E_{\hat{m}}{ }^{\hat{A}} \Sigma_{\hat{A} \hat{B} \hat{C} \hat{D} \hat{E}}\right| \\
& =\frac{1}{5 !} \varepsilon^{\hat{a} \hat{b} \hat{c} \hat{d} \hat{e}}\left(\Sigma_{\hat{a} \hat{b} \hat{c} \hat{d} \hat{e}}\left|+\frac{5}{2} \psi_{\hat{a} i}^{\hat{\alpha}} \Sigma_{\hat{b} \hat{c} \hat{d} \hat{e} \hat{\alpha}}^{i}\right|-\frac{5}{2} \psi_{\hat{a} i}{ }_{i}^{\hat{\alpha}} \psi_{\hat{b} j}^{\hat{\beta}} \Sigma_{\hat{c} \hat{d} \hat{e} \hat{e} \hat{\alpha}}^{i j} \mid\right. \\
& -\frac{5}{4} \psi_{\hat{a} i}^{\hat{\alpha}} \psi_{\hat{b} j}^{\hat{\beta}} \psi_{\hat{c} k}^{\hat{\gamma}} \Sigma_{\hat{d} \hat{e} \hat{\alpha} \hat{\beta} \hat{\gamma}}^{i j k}\left|+\frac{5}{16} \psi_{\hat{a} i}^{\hat{\alpha}} \psi_{\hat{b} j}^{\hat{\beta}} \psi_{\hat{c} k}^{\hat{\gamma}} \psi_{\hat{d} l}^{\hat{\delta}} \Sigma_{\hat{e} \hat{\alpha} \hat{\beta} \hat{\gamma} \hat{\delta}}^{i j k l}\right| \\
& \left.+\frac{1}{32} \psi_{\hat{a} i}^{\hat{\alpha}} \psi_{\hat{b} j}^{\hat{\beta}} \psi_{\hat{c} k}^{\hat{\gamma}} \psi_{\hat{d} l}^{\hat{\delta}} \psi_{\hat{e} p}^{\hat{\rho}} \sum_{\hat{\alpha} \hat{\beta} \hat{\gamma} \hat{\delta} \hat{\rho}}^{i j k l p} \mid\right) \text {. }
\end{aligned}
$$

The action is then

$$
\begin{aligned}
S= & \int \mathrm{d}^{5} x e\left(\frac{1}{4 !} \varepsilon^{\hat{m} \hat{n} \hat{p} \hat{q} \hat{r}} v_{\hat{m}} \phi_{\hat{n} \hat{p} \hat{q} \hat{r}}-W F-X^{i j} G_{i j}-2 \lambda^{k} \varphi_{k}\right. \\
& \left.+\psi_{\hat{m} i} \Gamma^{\hat{m}} \varphi^{i} W+\mathrm{i} \psi_{\hat{m} i} \Gamma^{\hat{m}} \lambda_{j} G^{i j}-\mathrm{i} \psi_{\hat{m} i} \Sigma^{\hat{m} \hat{n}} \psi_{\hat{n} j} W G^{i j}\right) \\
= & -\int \mathrm{d}^{5} x e\left(v^{\hat{a}} \phi_{\hat{a}}+W F+X^{i j} G_{i j}+2 \lambda^{k} \varphi_{k}\right. \\
& \left.-\psi_{\hat{a} i} \Gamma^{\hat{a}} \varphi^{i} W-\mathrm{i} \psi_{\hat{a} i} \Gamma^{\hat{a}} \lambda_{j} G^{i j}+\mathrm{i} \psi_{\hat{a} i} \Sigma^{\hat{a} \hat{b}} \psi_{\hat{b} j} W G^{i j}\right),
\end{aligned}
$$

${ }^{21}$ The Levi-Civita tensor with world indices is defined as $\varepsilon^{\hat{m} \hat{n} \hat{p} \hat{q} \hat{r}}:=\varepsilon^{\hat{a} \hat{b} \hat{c} \hat{d} \hat{e}} e_{\hat{a}}{ }^{\hat{m}} e_{\hat{b}}{ }^{\hat{n}} e_{\hat{c}}^{\hat{p}} e_{\hat{d}^{\hat{q}}} e_{\hat{e}}{ }^{\hat{r}}$. 
where all superfields appearing in the action are understood as their corresponding spacetime projections and we have defined

$$
\begin{array}{rlrl}
v_{\hat{a}} & :=e_{\hat{a}}^{\hat{m}} v_{\hat{m}}=e_{\hat{a}}^{\hat{m}} V_{\hat{m}} \mid, & \phi^{\hat{a}}:=-\frac{1}{4 !} e_{\hat{r}}^{\hat{a}} \varepsilon^{\hat{m} \hat{n} \hat{p} \hat{q} \hat{r}} \phi_{\hat{m} \hat{n} \hat{p} \hat{q}}, \\
\phi_{\hat{m} \hat{n} \hat{p} \hat{q}}:=\Phi_{\hat{m} \hat{n} \hat{p} \hat{q}} \mid & =4 \partial_{[\hat{m}} b_{\hat{m} \hat{n} \hat{p}]}, & b_{\hat{m} \hat{n} \hat{p}} & =\mathcal{B}_{\hat{m} \hat{n} \hat{p}} \mid .
\end{array}
$$

The Chern-Simons coupling between the one-form $V$ and the four-form $\Phi$ can equivalently be written

$$
\begin{aligned}
S= & \int \mathrm{d}^{5} x e\left(\frac{1}{12} \varepsilon^{\hat{a} \hat{b} \hat{c} \hat{d} \hat{e}} f_{\hat{a} \hat{b}} b_{\hat{c} \hat{d} \hat{e}}-W F-X^{i j} G_{i j}-2 \lambda^{k} \varphi_{k}\right. \\
& \left.+\psi_{\hat{a} i} \Gamma^{\hat{a}} \varphi^{i} W+\mathrm{i} \psi_{\hat{a} i} \Gamma^{\hat{a}} \lambda_{j} G^{i j}-\mathrm{i} \psi_{\hat{a} i} \Sigma^{\hat{a} \hat{b}} \psi_{\hat{b} j} W G^{i j}\right)
\end{aligned}
$$

where

$$
f_{\hat{a} \hat{b}}:=e_{\hat{a}}^{\hat{m}} e_{\hat{b}}^{\hat{n}} f_{\hat{m} \hat{n}}, \quad f_{\hat{m} \hat{n}}:=F_{\hat{m} \hat{n}} \mid=2 \partial_{[\hat{m}} v_{\hat{n}]}, \quad b_{\hat{a} \hat{b} \hat{c}}:=e_{\hat{a}}^{\hat{m}} e_{\hat{b}}^{\hat{n}} e_{\hat{c}}^{\hat{\hat{p}}} b_{\hat{m} \hat{n} \hat{p}} .
$$

It should be mentioned that the normalization of the action (8.28) has been chosen to correspond to the projective superspace action principle (5.35) with $\mathcal{L}^{(2)}=V G^{(2)}$. Furthermore, the action (8.28) corresponds to the BF action without central charge. We will give a generalization in the presence of a gauged central charge in section 11 .

\section{Abelian Chern-Simons theory}

In conformal superspace, the dynamics of an abelian vector multiplet coupled to conformal supergravity is described by the Chern-Simons action ${ }^{22}$

$$
S_{\mathrm{CS}}=\frac{1}{2 \pi} \oint_{\gamma}(v, \mathrm{~d} v) \int \mathrm{d}^{5 \mid 8} z E C^{(-4)} \mathcal{L}_{\mathrm{CS}}^{(2)}, \quad \mathcal{L}_{\mathrm{CS}}^{(2)}=-\frac{1}{12} V H_{\mathrm{VM}}^{(2)},
$$

where $H_{\mathrm{VM}}^{(2)}$ denotes the composite $\mathcal{O}(2)$ multiplet defined by (5.40). Varying the tropical prepotential gives

$$
\delta S_{\mathrm{CS}}=-\frac{1}{8 \pi} \oint(v, \mathrm{~d} v) \int \mathrm{d}^{5 \mid 8} z E C^{(-4)} \delta V H_{\mathrm{VM}}^{(2)},
$$

see section 12 for the derivation.

A component counterpart of the action (9.1) may be constructed using $H_{\mathrm{VM}}^{(2)}$ and the $\mathrm{BF}$ action (8.1). This amounts to plugging

$$
G^{i j}=H_{\mathrm{VM}}^{i j}=2 W X^{i j}-\mathrm{i} \lambda^{\hat{\alpha}(i} \lambda_{\hat{\alpha}}^{j)}
$$

into the component BF action (8.28) and computing the component fields of the composite $\mathcal{O}(2)$ multiplet. This produces the component $V \wedge F \wedge F$ coupling by treating the closed

\footnotetext{
${ }^{22}$ In the $5 \mathrm{D} \mathcal{N}=1$ super-Poincaré case, the off-shell abelian Chern-Simons action was constructed for the first time by Zupnik in harmonic superspace [51]. The action (9.1) is a curved-superspace extension of the one given in [2].
} 
gauge-invariant four-form $F \wedge F$ as the field strength derived from the $\mathcal{O}(2)$ multiplet (9.3). A major disadvantage of this approach is that the non-abelian Chern-Simons theory cannot be constructed in the same way. In this section we will discuss an alternative superform construction that can be generalized and show how to derive it explicitly from the BF action principle.

Recall that the BF action involved constructing a closed five-form $\mathfrak{J}$ given by

$$
\mathfrak{J}_{H}=V \wedge \Phi-\Sigma_{H},
$$

where $\Phi$ is the four-form field strength associated with the composite $G^{i j}$ and $\Sigma_{H}$, constructed in section 8.1, is a covariant four-form which solves the equation

$$
\mathrm{d} \Sigma_{H}=F \wedge \Phi
$$

If one now substitutes the relations

$$
\begin{aligned}
\varphi_{\hat{\alpha}}^{i}= & \mathrm{i} X^{i j} \lambda_{\hat{\alpha} j}-2 \mathrm{i} F_{\hat{\alpha} \hat{\beta}} \lambda^{\hat{\beta} i}+X_{\hat{\alpha}}^{i} W^{2}-2 \mathrm{i} W \nabla_{\hat{\alpha} \hat{\beta}} \lambda^{\hat{\beta} i}-\mathrm{i} \nabla_{\hat{\alpha} \hat{\beta}} W \lambda^{\hat{\beta} i} \\
F= & X^{i j} X_{i j}-F^{\hat{a} \hat{b}} F_{\hat{a} \hat{b}}+4 W \nabla^{\hat{a}} \nabla_{\hat{a}} W+2\left(\nabla^{\hat{a}} W\right) \nabla_{\hat{a}} W+2 \mathrm{i}\left(\nabla_{\hat{\alpha}}^{\hat{\beta}} \lambda_{\hat{\beta}}^{k}\right) \lambda_{k}^{\hat{\alpha}} \\
& -6 W^{\hat{a} \hat{b}} F_{\hat{a} \hat{b}} W-5 W^{\hat{a} \hat{b}} W_{\hat{a} \hat{b}} W^{2}+Y W^{2}+6 X^{\hat{\alpha} i} \lambda_{\hat{\alpha} i} W \\
\Phi_{\hat{a}}= & -\frac{1}{2} \varepsilon_{\hat{a} \hat{b} \hat{c} \hat{d} \hat{e}} F^{\hat{b} \hat{c}} F^{\hat{d} \hat{e}}+4 \nabla^{\hat{b}}\left(W F_{\hat{b} \hat{a}}+\frac{3}{2} W_{\hat{b} \hat{a}} W^{2}\right)+\varepsilon_{\hat{a} \hat{b} \hat{c} \hat{d} \hat{e}} W^{\hat{b} \hat{c}}\left(F^{\hat{d} \hat{e}}+\frac{3}{2} W^{\hat{d} \hat{e}} W\right) W \\
& -6\left(\Gamma_{\hat{a}}\right)^{\hat{\alpha} \hat{\beta}} X_{\hat{\alpha}}^{k} \lambda_{\hat{\beta} k} W+2 \mathrm{i}\left(\Sigma_{\hat{b} \hat{a}}\right)^{\hat{\alpha} \hat{\beta}} \nabla^{\hat{b}}\left(\lambda_{\hat{\alpha}}^{k} \lambda_{\hat{\beta} k}\right)+\frac{\mathrm{i}}{2} \varepsilon_{\hat{a} \hat{b} \hat{c} \hat{d} \hat{e}} W^{\hat{b} \hat{c}}\left(\Sigma^{\hat{d} \hat{e}}\right)^{\hat{\alpha} \hat{\beta}} \lambda_{\hat{\alpha}}^{k} \lambda_{\hat{\beta} k}
\end{aligned}
$$

into the eqs. (8.6) defining the superform $\Phi$ and (8.22) for $\Sigma_{H}$, one arrives at the abelian CS action. However, as is evident from considering the expression for $\Phi_{\hat{a}}$ above, the expression involves several derivatives which must be integrated by parts to arrive at the conventional form of the action.

We seek instead a different closed superform $\mathfrak{J}$, which will be given by

$$
\mathfrak{J}=\Sigma_{\mathrm{CS}}-\Sigma_{R},
$$

where both $\Sigma_{\mathrm{CS}}$ and $\Sigma_{R}$ are solutions to the equation

$$
\mathrm{d} \Sigma=F \wedge F \wedge F
$$

The first is the Chern-Simons form,

$$
\Sigma_{\mathrm{CS}}=V \wedge F \wedge F,
$$

while the second, $\Sigma_{R}$, we will refer to as the curvature induced form. Here the curvature induced form is required to be a gauge-invariant primary superform constructed directly out of $W$ and its covariant derivatives. The Chern-Simons and curvature induced forms represent ingredients in a general procedure to construct Chern-Simons actions in three and five dimensions, see $[48,49,75,76]$. Gauge invariance of the corresponding action (8.11) is guaranteed by the fact that $\Sigma_{R}$ is gauge invariant by construction, while $\Sigma_{\mathrm{CS}}$ 
transforms via an exact form. The advantage of this construction over the use of the BF action is that it can be straightforwardly generalized to the non-abelian case.

Now it turns out that $\mathfrak{J}_{H}$ and $2 \mathfrak{J}$ describe the same component action, with $2 \mathfrak{J}$ differing from $\mathfrak{J}$ by a total derivative (i.e. by an exact form) alluded to above. In other words,

$$
\mathrm{d} \mathfrak{V}=2 \mathfrak{J}-\mathfrak{J}_{H}=V \wedge(2 F \wedge F-\Phi)-2 \Sigma_{R}+\Sigma_{H},
$$

for some four-form $\mathfrak{V}$. It is evident we can choose

$$
\mathfrak{V}=V \wedge C,
$$

for some three-form $C$ satisfying

$$
\mathrm{d} C=2 F \wedge F-\Phi .
$$

Provided there exists a gauge-invariant primary three-form $C$ that solves this equation, then the curvature induced form is given immediately as

$$
\Sigma_{R}=\frac{1}{2}\left(\Sigma_{H}+F \wedge C\right) .
$$

The construction of such a three-form $C$ is straightforward. From dimensional considerations, it is evident that $C_{\hat{\alpha} \hat{\beta} \hat{\gamma}}^{i j k}$ must vanish, while $C_{\hat{a}}^{j k} j_{\hat{\beta} \hat{\gamma}}$ must be proportional to $W^{2}$. The full solution is straightforward to derive:

$$
\begin{aligned}
C_{\hat{a}}^{j k} \hat{\beta} \hat{\gamma} & =-4 \mathrm{i}\left(\Gamma_{\hat{a}}\right)_{\hat{\beta} \hat{\gamma}} \varepsilon^{j k} W^{2}, \\
C_{\hat{a} \hat{b} \hat{\gamma}}^{k} & =8 \mathrm{i}\left(\Sigma_{\hat{a} \hat{b}}\right) \hat{\gamma}^{\hat{\gamma}} \lambda_{\hat{\delta}}^{k} W, \\
C_{\hat{a} \hat{b} \hat{c}} & =\varepsilon_{\hat{a} \hat{b} \hat{c} \hat{d} \hat{e}}\left(2 F^{\hat{d} \hat{e}} W+\mathrm{i} \lambda_{k} \Sigma^{\hat{d} \hat{e}} \lambda^{k}+3 W^{\hat{d} \hat{e}} W^{2}\right) .
\end{aligned}
$$

The construction of $\Sigma_{R}$ is now immediate. As required, it is given purely in terms of $W$ and its covariant derivatives, with the nonzero components given below: ${ }^{23}$

$$
\begin{aligned}
& \Sigma_{\hat{a} \hat{\alpha} \hat{\beta} \hat{\gamma} \hat{\delta}}^{i j k l}=4\left(\varepsilon^{i j} \varepsilon^{k l}\left(\left(\Gamma_{\hat{a}}\right)_{\hat{\alpha} \hat{\beta}} \varepsilon_{\hat{\gamma} \hat{\delta}}+\left(\Gamma_{\hat{a}}\right)_{\hat{\gamma} \hat{\delta}} \varepsilon_{\hat{\alpha} \hat{\beta}}\right)+\varepsilon^{i k} \varepsilon^{j l}\left(\left(\Gamma_{\hat{a}}\right)_{\hat{\alpha} \hat{\gamma}} \varepsilon_{\hat{\beta} \hat{\delta}}+\left(\Gamma_{\hat{a}}\right)_{\hat{\beta} \hat{\delta}} \varepsilon_{\hat{\alpha} \hat{\gamma}}\right)\right. \\
& \left.+\varepsilon^{i l} \varepsilon^{j k}\left(\left(\Gamma_{\hat{a}}\right)_{\hat{\alpha} \hat{\delta}} \varepsilon_{\hat{\beta} \hat{\gamma}}+\left(\Gamma_{\hat{a}}\right)_{\hat{\beta} \hat{\gamma}} \varepsilon_{\hat{\alpha} \hat{\delta}}\right)\right) W^{3}, \\
& \left.\Sigma_{\hat{a} \hat{b} \hat{\alpha} \hat{\beta} \hat{\gamma}}^{i j k}=-12\left(\varepsilon^{j k} \varepsilon_{\hat{\beta} \hat{\gamma}}\left(\Sigma_{\hat{a} \hat{b}}\right) \hat{\alpha}_{\hat{\alpha}}^{\hat{\delta}} \lambda_{\hat{\delta}}^{i} W^{2}+\varepsilon^{i j} \varepsilon_{\hat{\alpha} \hat{\beta}}\left(\Sigma_{\hat{a} \hat{b}}\right)\right)_{\hat{\gamma}}^{\hat{\delta}} \lambda_{\hat{\delta}}^{k} W^{2}+\varepsilon^{i k} \varepsilon_{\hat{\gamma} \hat{\alpha}}\left(\Sigma_{\hat{a} \hat{b}}\right)_{\hat{\beta}}^{\hat{\delta}} \lambda_{\hat{\delta}}^{j} W^{2}\right) \\
& +8\left(\varepsilon^{j k}\left(\Sigma_{\hat{a} \hat{b}}\right)_{\hat{\alpha} \hat{\beta}} \lambda_{\hat{\gamma}}^{i} W^{2}+\varepsilon^{k i}\left(\Sigma_{\hat{a} \hat{b}}\right)_{\hat{\beta} \hat{\gamma}} \lambda_{\hat{\alpha}}^{j} W^{2}+\varepsilon^{i j}\left(\Sigma_{\hat{a} \hat{b}}\right) \hat{\gamma} \hat{\alpha} \lambda_{\hat{\beta}}^{k} W^{2}\right), \\
& \Sigma_{\hat{a} \hat{b} \hat{b} \hat{c} \hat{\alpha}}^{i j}=\frac{\mathrm{i}}{2} \varepsilon^{i j} \varepsilon_{\hat{\alpha} \hat{\beta}} \varepsilon_{\hat{a} \hat{b} \hat{c} \hat{d} \hat{e}}\left(\Sigma^{\hat{d} \hat{e}}\right)^{\hat{\gamma} \hat{\delta}}\left(4 W^{2} F_{\hat{\gamma} \hat{\delta}}+5 \mathrm{i} W \lambda_{\hat{\gamma}}^{k} \lambda_{\hat{\delta} k}+6 W_{\hat{\gamma} \hat{\delta}} W^{3}\right) \\
& -\mathrm{i} \varepsilon_{\hat{a} \hat{b} \hat{c} \hat{d} \hat{e}}\left(\Sigma^{\hat{d} \hat{e}}\right)_{\hat{\alpha} \hat{\beta}}\left(4 W^{2} X^{i j}-5 \mathrm{i} W \lambda^{\hat{\gamma}(i} \lambda_{\hat{\gamma}}^{j)}\right) \\
& +6\left(\Sigma_{[\hat{a} \hat{b}}\right)_{\hat{\alpha} \hat{\beta}}\left(\Gamma_{\hat{c}}\right)^{\hat{\gamma} \hat{\delta}} \lambda_{\hat{\gamma}}^{(i} \lambda_{\hat{\delta}}^{j)} W+3 \varepsilon^{i j}\left(\Gamma_{[\hat{a}}\right)_{\hat{\alpha} \hat{\beta}}\left(\Sigma_{\hat{b} \hat{c}]}\right)^{\hat{\gamma} \hat{\delta}} \lambda_{\hat{\gamma}}^{k} \lambda_{\hat{\delta} k} W \\
& -6 \mathrm{i} \varepsilon^{i j}\left(\Gamma_{[\hat{a}}\right)_{\hat{\alpha} \hat{\beta}} F_{\hat{b} \hat{c}]} W^{2} \text {, }
\end{aligned}
$$

\footnotetext{
${ }^{23}$ We drop the subscript $R$ when referring to the components of $\Sigma_{R}$ to avoid awkward notation.
} 


$$
\begin{aligned}
\Sigma_{\hat{a} \hat{b} \hat{c} \hat{d} \hat{\alpha}}= & -\frac{1}{2} \varepsilon_{\hat{a} \hat{b} \hat{c} \hat{d} \hat{e}}\left(\Gamma^{\hat{e}}\right)_{\hat{\alpha}}^{\hat{\beta}}\left(12 \mathrm{i} W F_{\hat{\beta} \hat{\gamma}} \lambda^{\hat{\gamma} i}-6 \mathrm{i} W X^{i j} \lambda_{\hat{\beta} j}-4 \lambda^{\hat{\gamma}(i} \lambda_{\hat{\gamma}}^{j)} \lambda_{\hat{\beta} j}\right. \\
& \left.-2 X_{\hat{\beta}}^{i} W^{3}+6 \mathrm{i} W_{\hat{\beta} \hat{\gamma}} W^{2} \lambda^{\hat{\gamma} i}\right) \\
& +\varepsilon_{\hat{a} \hat{b} \hat{c} \hat{d} \hat{e}}\left(\Gamma^{\hat{e}}\right)^{\hat{\beta} \hat{\gamma}} \operatorname{tr}\left(\lambda_{\hat{\beta}}^{(i} \lambda_{\hat{\gamma}}^{j)} \lambda_{\hat{\alpha} j}+3 \mathrm{i} W_{\hat{\alpha} \hat{\beta}} W^{2} \lambda_{\hat{\gamma}}^{i}\right) \\
& -\mathrm{i} \varepsilon_{\hat{a} \hat{b} \hat{c} \hat{d} \hat{e}} W \lambda_{\hat{\alpha}}^{i} \nabla^{\hat{e}} W-2 \mathrm{i} \varepsilon_{\hat{a} \hat{b} \hat{c} \hat{d} \hat{e}} W^{2} \nabla^{\hat{e}} \lambda_{\hat{\alpha}}^{i} \\
& -2 \mathrm{i} \varepsilon_{\hat{a} \hat{b} \hat{c} \hat{d} \hat{e}}\left(\sum^{\hat{e} \hat{f}}\right)_{\hat{\alpha}}^{\hat{\beta}} W \lambda_{\hat{\beta}}^{i} \nabla_{\hat{f}} W-4 \mathrm{i} \varepsilon_{\hat{a} \hat{b} \hat{c} \hat{d} \hat{e}}\left(\Sigma^{\hat{e} \hat{f}}\right)_{\hat{\alpha}}^{\hat{\beta}} W^{2} \nabla_{\hat{f}} \lambda_{\hat{\beta}}^{i}, \\
\Sigma_{\hat{a} \hat{b} \hat{c} \hat{d} \hat{e}}= & -\frac{3}{2} \varepsilon_{\hat{a} \hat{b} \hat{c} \hat{d} \hat{e}}\left(W X^{k l} X_{k l}-2 W F^{\hat{\gamma} \hat{\delta}} F_{\hat{\gamma} \hat{\delta}}-\mathrm{i} X^{k l} \lambda_{k}^{\hat{\delta}} \lambda_{\hat{\delta} l}-2 \mathrm{i} F^{\hat{\gamma} \hat{\delta}} \lambda_{\hat{\gamma}}^{k} \lambda_{\hat{\delta} k}\right. \\
& +\frac{2}{3} W\left(\nabla^{\hat{f}} W\right) \nabla_{\hat{f}} W+\frac{4}{3} W^{2} \nabla^{\hat{f}} \nabla_{\hat{f}} W+2 \mathrm{i} W\left(\nabla_{\hat{\gamma} \hat{\delta}} \lambda^{\hat{\gamma} k}\right) \lambda_{k}^{\hat{\delta}} \\
& \left.+\frac{1}{3} Y W^{3}-\frac{4}{3} X^{\hat{\gamma} k} \lambda_{\hat{\gamma} k} W^{2}-6 W^{\hat{\gamma} \hat{\delta}} F_{\hat{\gamma} \hat{\delta}} W^{2}-\frac{10}{3} W^{\hat{\gamma} \hat{\delta}} W_{\hat{\gamma} \hat{\delta}} W^{3}\right) .
\end{aligned}
$$

The abelian Chern-Simons action is then given by

$$
S_{\mathrm{CS}}=-\frac{1}{6} \int_{\mathcal{M}^{5}} i^{*} \mathfrak{J},
$$

where we have adjusted the normalization to match (9.1). As we will show in the next section, it is straightforward to generalize the result for $\mathfrak{J}$ to a non-abelian vector multiplet. We will give the explicit component action in the next section for the non-abelian case.

\section{Non-abelian Chern-Simons theory}

In the non-abelian case, a closed-form expression for the Chern-Simons action as a superspace integral is not yet known. However, the corresponding action may be defined by postulating its variation ${ }^{24}$

$$
\delta S_{\mathrm{CS}}=-\frac{1}{8 \pi} \oint_{\gamma}(v, \mathrm{~d} v) \int \mathrm{d}^{5 \mid 8} z E C^{(-4)} \operatorname{tr}\left(\Delta V \cdot \mathrm{e}^{-\Omega_{+}} \boldsymbol{H}_{\mathrm{YM}}^{(2)} \mathrm{e}^{\Omega_{+}}\right)
$$

where we have defined

$$
\Delta V:=\mathrm{e}^{-V} \delta \mathrm{e}^{V}, \quad \boldsymbol{H}_{\mathrm{YM}}^{(2)}=v_{i} v_{i} \boldsymbol{H}_{\mathrm{YM}}^{i j},
$$

with the composite superfield $\boldsymbol{H}_{\mathrm{YM}}^{i j}$ given by (2.54). Here $\Delta V$ is the covariantized variation of the tropical prepotential. In the abelian case, the variation (10.1) reduces to (9.2). In this paper, we will not elaborate on the above definition, and instead present a superform realization of the action.

In the previous section we derived the closed five-form describing the abelian ChernSimons theory and introduced two key ingredients: the Chern-Simons and curvature induced forms. In this section we will show how to generalize our approach to the non-abelian

\footnotetext{
${ }^{24}$ This definition is inspired by the earlier works [51, 65, 78].
} 
Chern-Simons theory based on a Yang-Mills multiplet and derive the corresponding component action. Our approach is analogous to the one adopted in [77] in Minkowski superspace. We remind the reader that the Yang-Mills multiplet is described in section 2.6.

The appropriate closed five-form $\mathfrak{J}$ to describe the non-abelian Chern-Simons action may be found by generalizing the Chern-Simons form and the curvature induced form. These five-forms now correspond to two solutions of the superform equation

$$
\mathrm{d} \Sigma=\left\langle\boldsymbol{F}^{3}\right\rangle:=\operatorname{tr}(\boldsymbol{F} \wedge \boldsymbol{F} \wedge \boldsymbol{F}),
$$

which is a straightforward generalization of (9.8). The Chern-Simons form $\Sigma_{\mathrm{CS}}$ is again directly constructed out of $\boldsymbol{V}$, while the curvature induced form is constructed out of $\boldsymbol{W}$ and its covariant derivatives. If they transform by an exact form under the gauge group then their difference

$$
\mathfrak{J}=\Sigma_{\mathrm{CS}}-\Sigma_{R}
$$

will yield an appropriate closed five-form that describes the action. The Chern-Simons and curvature induced five-forms are discussed in more detail below.

\subsection{The Chern-Simons five-form}

The Chern-Simons form is

$$
\Sigma_{\mathrm{CS}}=\operatorname{tr}\left(\boldsymbol{V} \wedge \boldsymbol{F} \wedge \boldsymbol{F}-\frac{\mathrm{i}}{2} \boldsymbol{V} \wedge \boldsymbol{V} \wedge \boldsymbol{V} \wedge \boldsymbol{F}-\frac{1}{10} \boldsymbol{V} \wedge \boldsymbol{V} \wedge \boldsymbol{V} \wedge \boldsymbol{V} \wedge \boldsymbol{V}\right) .
$$

One can verify that it satisfies the superform equation (10.2) by using

$$
\boldsymbol{\nabla}=\mathrm{d}-\mathrm{i} \boldsymbol{V}, \quad \boldsymbol{F}=\mathrm{d} \boldsymbol{V}+\mathrm{i} \boldsymbol{V} \wedge \boldsymbol{V}, \quad \boldsymbol{\nabla} \boldsymbol{F}=0 \Longrightarrow \mathrm{d} \boldsymbol{F}=\mathrm{i} \boldsymbol{V} \wedge \boldsymbol{F}-\mathrm{i} \boldsymbol{F} \wedge \boldsymbol{V} .
$$

Since $\Sigma_{\mathrm{CS}}$ has been constructed by extracting a total derivative from the gauge invariant superform $\left\langle\boldsymbol{F}^{3}\right\rangle$ it must transform by a closed form under the gauge group. In fact, one can show it transforms by an exact form,

$$
\Sigma_{\mathrm{CS}} \rightarrow \Sigma_{\mathrm{CS}}+\mathrm{d} \operatorname{tr}\left(\mathrm{d} \tau \wedge\left(\boldsymbol{V} \wedge \boldsymbol{F}-\frac{\mathrm{i}}{2} \boldsymbol{V} \wedge \boldsymbol{V} \wedge \boldsymbol{V}\right)\right)
$$

Note that since the gauge field $\boldsymbol{V}$ is primary, $\Sigma_{\mathrm{CS}}$ is also a primary superform.

\subsection{The curvature-induced five-form}

To construct the curvature-induced five-form we look for a gauge-invariant solution to

$$
\mathrm{d} \Sigma=\operatorname{tr}(\boldsymbol{F} \wedge \boldsymbol{F} \wedge \boldsymbol{F}) .
$$

The condition that $\Sigma$ is invariant allows one to express eq. (10.7) as

$$
2 \boldsymbol{\nabla}_{[\hat{A}} \Sigma_{\hat{B} \hat{C} \hat{D} \hat{E} \hat{F}\}}+5 \mathscr{T}_{[\hat{A} \hat{B}}{ }^{\hat{G}} \Sigma_{|\hat{G}| \hat{C} \hat{D} \hat{E} \hat{F}\}}=30 \operatorname{tr}\left(\boldsymbol{F}_{[\hat{A} \hat{B}} \boldsymbol{F}_{\hat{C} \hat{D}} \boldsymbol{F}_{\hat{E} \hat{F}\}}\right) .
$$

Here we have used the fact that $\Sigma$ is a gauge singlet ${ }^{25}$

$$
\nabla_{\hat{A}} \Sigma_{\hat{B} \hat{C} \hat{D} \hat{E} \hat{F}}=\nabla_{\hat{A}} \Sigma_{\hat{B} \hat{C} \hat{D} \hat{E} \hat{F}}
$$

\footnotetext{
${ }^{25}$ Keeping this in mind, we will use gauge covariant derivatives everywhere in this section.
} 
The curvature induced form is defined to be a primary solution of eq. (10.7) that can be expressed covariantly in terms of the vector multiplet field strength $\boldsymbol{W}$. Invariance of the curvature induced form is then guaranteed. On dimensional grounds, it is natural to impose the constraint

$$
\Sigma_{\hat{\alpha} \hat{\beta} \hat{\gamma} \hat{\delta} \hat{\rho}}^{i j k l p}=0
$$

Then analyzing the superform equation (10.7) by increasing dimension, enforcing the primary condition (8.18) and using the identities (2.52) yields all the remaining components of the curvature induced five-form:

$$
\begin{aligned}
& \Sigma_{\hat{a} \hat{\alpha} \hat{\beta} \hat{\gamma} \hat{\delta}}^{i j k l}=4\left(\varepsilon^{i j} \varepsilon^{k l}\left(\left(\Gamma_{\hat{a}}\right)_{\hat{\alpha} \hat{\beta}} \varepsilon_{\hat{\gamma} \hat{\delta}}+\left(\Gamma_{\hat{a}}\right)_{\hat{\gamma} \hat{\delta}} \varepsilon_{\hat{\alpha} \hat{\beta}}\right)+\varepsilon^{i k} \varepsilon^{j l}\left(\left(\Gamma_{\hat{a}}\right)_{\hat{\alpha} \hat{\gamma}} \varepsilon_{\hat{\beta} \hat{\delta}}+\left(\Gamma_{\hat{a}}\right)_{\hat{\beta} \hat{\delta}} \varepsilon_{\hat{\alpha} \hat{\gamma}}\right)\right. \\
& \left.+\varepsilon^{i l} \varepsilon^{j k}\left(\left(\Gamma_{\hat{a}}\right)_{\hat{\alpha} \hat{\delta}} \varepsilon_{\hat{\beta} \hat{\gamma}}+\left(\Gamma_{\hat{a}}\right)_{\hat{\beta} \hat{\gamma}} \varepsilon_{\hat{\alpha} \hat{\delta}}\right)\right) \operatorname{tr}\left(\boldsymbol{W}^{3}\right), \\
& \Sigma_{\hat{a} \hat{b} \hat{\alpha} \hat{\beta} \hat{\gamma}}^{i j k}=-12 \operatorname{tr}\left(\varepsilon^{j k} \varepsilon_{\hat{\beta} \hat{\gamma}}\left(\Sigma_{\hat{a} \hat{b}}\right) \hat{\alpha}_{\hat{\alpha}}^{\hat{\delta}} \boldsymbol{\lambda}_{\hat{\delta}}^{i} \boldsymbol{W}^{2}+\varepsilon^{i j} \varepsilon_{\hat{\alpha} \hat{\beta}}\left(\Sigma_{\hat{a} \hat{b}}\right)_{\hat{\gamma}}^{\hat{\delta}} \boldsymbol{\lambda}_{\hat{\delta}}^{k} \boldsymbol{W}^{2}+\varepsilon^{i k} \varepsilon_{\hat{\gamma} \hat{\alpha}}\left(\Sigma_{\hat{a} \hat{b}}\right)_{\hat{\beta}} \hat{\delta} \boldsymbol{\lambda}_{\hat{\delta}}^{j} \boldsymbol{W}^{2}\right) \\
& +8 \operatorname{tr}\left(\varepsilon^{j k}\left(\Sigma_{\hat{a} \hat{b}}\right)_{\hat{\alpha} \hat{\beta}} \boldsymbol{\lambda}_{\hat{\gamma}}^{i} \boldsymbol{W}^{2}+\varepsilon^{k i}\left(\Sigma_{\hat{a} \hat{b}}\right)_{\hat{\beta} \hat{\gamma}} \boldsymbol{\lambda}_{\hat{\alpha}}^{j} \boldsymbol{W}^{2}+\varepsilon^{i j}\left(\Sigma_{\hat{a} \hat{b}}\right) \hat{\gamma} \hat{\alpha} \boldsymbol{\lambda}_{\hat{\beta}}^{k} \boldsymbol{W}^{2}\right), \\
& \Sigma_{\hat{a} \hat{b} \hat{c} \hat{\alpha} \hat{\beta}}^{i j}=\frac{i}{2} \varepsilon^{i j} \varepsilon_{\hat{\alpha} \hat{\beta}} \varepsilon_{\hat{a} \hat{b} \hat{c} \hat{d} \hat{e}}\left(\Sigma^{\hat{d e} \hat{e}}\right)^{\hat{\gamma} \hat{\delta}} \operatorname{tr}\left(4 \boldsymbol{W}^{2} \boldsymbol{F}_{\hat{\gamma} \hat{\delta}}+5 \mathrm{i} \boldsymbol{W} \boldsymbol{\lambda}_{\hat{\gamma}}^{k} \boldsymbol{\lambda}_{\hat{\delta} k}+6 W_{\hat{\gamma} \hat{\delta}} \boldsymbol{W}^{3}\right) \\
& -\mathrm{i} \varepsilon_{\hat{a} \hat{b} \hat{c} \hat{d} \hat{e}}\left(\Sigma^{\hat{d} \hat{e}}\right)_{\hat{\alpha} \hat{\beta}} \operatorname{tr}\left(4 \boldsymbol{W}^{2} \boldsymbol{X}^{i j}-5 \mathrm{i} \boldsymbol{W} \boldsymbol{\lambda}^{\hat{\gamma}(i} \boldsymbol{\lambda}_{\hat{\gamma}}^{j)}\right) \\
& +6\left(\Sigma_{[\hat{a} \hat{b}}\right)_{\hat{\alpha} \hat{\beta}}\left(\Gamma_{\hat{c}]}\right)^{\hat{\gamma} \hat{\delta}} \operatorname{tr}\left(\boldsymbol{\lambda}_{\hat{\gamma}}^{(i} \boldsymbol{\lambda}_{\hat{\delta}}^{j)} \boldsymbol{W}\right)+3 \varepsilon^{i j}\left(\Gamma_{[\hat{a}}\right)_{\hat{\alpha} \hat{\beta}}\left(\Sigma_{\hat{b} \hat{c}]}\right)^{\hat{\gamma} \hat{\delta}} \operatorname{tr}\left(\boldsymbol{\lambda}_{\hat{\gamma}}^{k} \boldsymbol{\lambda}_{\hat{\delta} k} \boldsymbol{W}\right) \\
& -6 \mathbf{i} \varepsilon^{i j}\left(\Gamma_{[\hat{a}}\right)_{\hat{\alpha} \hat{\beta}} \operatorname{tr}\left(\boldsymbol{F}_{\hat{b} \hat{c}]} \boldsymbol{W}^{2}\right) \text {, } \\
& \Sigma_{\hat{a} \hat{b} \hat{b} \hat{d} \hat{\alpha}}^{i}=-\frac{1}{2} \varepsilon_{\hat{a} \hat{b} \hat{c} \hat{d} \hat{e}}\left(\Gamma^{\hat{e}}\right)_{\hat{\alpha}}^{\hat{\beta}} \operatorname{tr}\left(6 \mathrm{i} \boldsymbol{W}\left\{\boldsymbol{F}_{\hat{\beta} \hat{\gamma}}, \boldsymbol{\lambda}^{\hat{\gamma} i}\right\}-3 \mathrm{i} \boldsymbol{W}\left\{\boldsymbol{X}^{i j}, \boldsymbol{\lambda}_{\hat{\beta} j}\right\}\right. \\
& \left.-4 \boldsymbol{\lambda}^{\hat{\gamma}(i} \boldsymbol{\lambda}_{\hat{\gamma}}^{j)} \boldsymbol{\lambda}_{\hat{\beta} j}-2 X_{\hat{\beta}}^{i} \boldsymbol{W}^{3}+6 \mathrm{i} W_{\hat{\beta} \hat{\gamma}} \boldsymbol{W}^{2} \boldsymbol{\lambda}^{\hat{\gamma} i}\right) \\
& +\varepsilon_{\hat{a} \hat{b} \hat{c} \hat{d} \hat{e}}\left(\Gamma^{\hat{e}}\right)^{\hat{\beta} \hat{\gamma}} \operatorname{tr}\left(\boldsymbol{\lambda}_{\hat{\beta}}^{(i} \boldsymbol{\lambda}_{\hat{\gamma}}^{j)} \boldsymbol{\lambda}_{\hat{\alpha} j}+3 \mathrm{i} W_{\hat{\alpha} \hat{\beta}} \boldsymbol{W}^{2} \boldsymbol{\lambda}_{\hat{\gamma}}^{i}\right) \\
& -\frac{\mathrm{i}}{2} \varepsilon_{\hat{a} \hat{b} \hat{c} \hat{d} \hat{e}} \operatorname{tr}\left(\left\{\boldsymbol{W}, \boldsymbol{\nabla}^{\hat{e}} \boldsymbol{W}\right\} \boldsymbol{\lambda}_{\hat{\alpha}}^{i}\right)-2 \mathrm{i} \varepsilon_{\hat{a} \hat{b} \hat{c} \hat{d} \hat{e}} \operatorname{tr}\left(\boldsymbol{W}^{2} \boldsymbol{\nabla}^{\hat{e}} \boldsymbol{\lambda}_{\hat{\alpha}}^{i}\right) \\
& -\mathrm{i} \varepsilon_{\hat{a} \hat{b} \hat{c} \hat{d} \hat{e}}\left(\Sigma^{\hat{e} \hat{f}}\right)_{\hat{\alpha}}^{\hat{\beta}} \operatorname{tr}\left(\left\{\boldsymbol{W}, \boldsymbol{\nabla}_{\hat{f}} \boldsymbol{W}\right\} \boldsymbol{\lambda}_{\hat{\beta}}^{i}\right)-4 \mathrm{i} \varepsilon_{\hat{a} \hat{b} \hat{c} \hat{d} \hat{e}}\left(\Sigma^{\hat{e} \hat{f}}\right)_{\hat{\alpha}}^{\hat{\beta}} \operatorname{tr}\left(\boldsymbol{W}^{2} \boldsymbol{\nabla}_{\hat{f}} \boldsymbol{\lambda}_{\hat{\beta}}^{i}\right), \\
& \Sigma_{\hat{a} \hat{b} \hat{c} \hat{d} \hat{e}}=-\frac{3}{2} \varepsilon_{\hat{a} \hat{b} \hat{c} \hat{d} \hat{e}} \operatorname{tr}\left(\boldsymbol{W} \boldsymbol{X}^{k l} \boldsymbol{X}_{k l}-2 \boldsymbol{W} \boldsymbol{F}^{\hat{\gamma} \hat{\delta}} \boldsymbol{F}_{\hat{\gamma} \hat{\delta}}-\mathrm{i} \boldsymbol{X}^{k l} \boldsymbol{\lambda}_{k}^{\hat{\delta}} \boldsymbol{\lambda}_{\hat{\delta} l}-2 \mathrm{i} \boldsymbol{F}^{\hat{\gamma} \hat{\delta}} \boldsymbol{\lambda}_{\hat{\gamma}}^{k} \boldsymbol{\lambda}_{\hat{\delta} k}\right. \\
& +\frac{2}{3} \boldsymbol{W}\left(\boldsymbol{\nabla}^{\hat{f}} \boldsymbol{W}\right) \boldsymbol{\nabla}_{\hat{f}} \boldsymbol{W}+\frac{4}{3} \boldsymbol{W}^{2} \boldsymbol{\nabla}^{\hat{f}} \boldsymbol{\nabla}_{\hat{f}} \boldsymbol{W}+\mathrm{i} \boldsymbol{W}\left[\boldsymbol{\nabla}_{\hat{\gamma} \hat{\delta}} \boldsymbol{\lambda}^{\hat{\gamma} k}, \boldsymbol{\lambda}_{k}^{\hat{\delta}}\right]-2 \boldsymbol{W}^{2} \boldsymbol{\lambda}^{\hat{\gamma} k} \boldsymbol{\lambda}_{\hat{\gamma} k} \\
& \left.+\frac{1}{3} Y \boldsymbol{W}^{3}-\frac{4}{3} X^{\hat{\gamma} k} \boldsymbol{\lambda}_{\hat{\gamma} k} \boldsymbol{W}^{2}-6 W^{\hat{\gamma} \hat{\delta}} \boldsymbol{F}_{\hat{\gamma} \hat{\delta}} \boldsymbol{W}^{2}-\frac{10}{3} W^{\hat{\gamma} \hat{\delta}} W_{\hat{\gamma} \hat{\delta}} \boldsymbol{W}^{3}\right) .
\end{aligned}
$$

It is worth elucidating the relation of the above curvature induced form to the one constructed in [76] in the rigid supersymmetric case. Switching off the Weyl multiplet $\left(W_{\hat{\alpha} \hat{\beta}}=0\right)$ and replacing the covariant derivatives with their corresponding flat ones,

$$
\nabla_{\hat{A}} \rightarrow \mathcal{D}_{\hat{A}}=\mathcal{D}_{\hat{A}}-\mathrm{i} \boldsymbol{V}_{\hat{A}},
$$


gives

$$
\begin{aligned}
& \Sigma_{\hat{a} \hat{\alpha} \hat{\beta} \hat{\gamma} \hat{\delta}}^{i j k l}=4\left(\varepsilon^{i j} \varepsilon^{k l}\left(\left(\Gamma_{\hat{a}}\right)_{\hat{\alpha} \hat{\beta}} \varepsilon_{\hat{\gamma} \hat{\delta}}+\left(\Gamma_{\hat{a}}\right)_{\hat{\gamma} \hat{\delta}} \varepsilon_{\hat{\alpha} \hat{\beta}}\right)+\varepsilon^{i k} \varepsilon^{j l}\left(\left(\Gamma_{\hat{a}}\right)_{\hat{\alpha} \hat{\gamma}} \varepsilon_{\hat{\beta} \hat{\delta}}+\left(\Gamma_{\hat{a}}\right)_{\hat{\beta} \hat{\delta}} \varepsilon_{\hat{\alpha} \hat{\gamma}}\right)\right. \\
& \left.+\varepsilon^{i l} \varepsilon^{j k}\left(\left(\Gamma_{\hat{a}}\right)_{\hat{\alpha} \hat{\delta}} \varepsilon_{\hat{\beta} \hat{\gamma}}+\left(\Gamma_{\hat{a}}\right)_{\hat{\beta} \hat{\gamma}} \varepsilon_{\hat{\alpha} \hat{\delta}}\right)\right) \operatorname{tr}\left(\boldsymbol{W}^{3}\right) \text {, } \\
& \Sigma_{\hat{a} \hat{b} \hat{\alpha} \hat{\beta} \hat{\gamma}}^{i j k}=-12 \operatorname{tr}\left(\varepsilon^{j k} \varepsilon_{\hat{\beta} \hat{\gamma}}\left(\Sigma_{\hat{a} \hat{b}}\right) \hat{\alpha} \boldsymbol{\lambda}_{\hat{\delta}}^{\hat{\delta}} \boldsymbol{W}^{2}+\varepsilon^{i j} \varepsilon_{\hat{\alpha} \hat{\beta}}\left(\Sigma_{\hat{a} \hat{b}}\right)_{\hat{\gamma}}^{\hat{\delta}} \boldsymbol{\lambda}_{\hat{\delta}}^{k} \boldsymbol{W}^{2}+\varepsilon^{i k} \varepsilon_{\hat{\gamma} \hat{\alpha}}\left(\Sigma_{\hat{a} \hat{b}}\right)_{\hat{\beta}}^{\hat{\delta}} \boldsymbol{\lambda}_{\hat{\delta}}^{j} \boldsymbol{W}^{2}\right) \\
& +8 \operatorname{tr}\left(\varepsilon^{j k}\left(\Sigma_{\hat{a} \hat{b}}\right)_{\hat{\alpha} \hat{\beta}} \boldsymbol{\lambda}_{\hat{\gamma}}^{i} \boldsymbol{W}^{2}+\varepsilon^{k i}\left(\Sigma_{\hat{a} \hat{b}}\right)_{\hat{\beta} \hat{\gamma}} \boldsymbol{\lambda}_{\hat{\alpha}}^{j} \boldsymbol{W}^{2}+\varepsilon^{i j}\left(\Sigma_{\hat{a} \hat{b}}\right) \hat{\gamma} \hat{\alpha} \boldsymbol{\lambda}_{\hat{\beta}}^{k} \boldsymbol{W}^{2}\right) \text {, }
\end{aligned}
$$

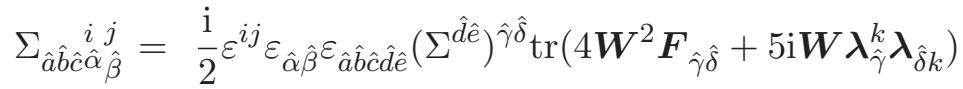

$$
\begin{aligned}
& -\mathrm{i} \varepsilon_{\hat{a} \hat{b} \hat{c} \hat{d} \hat{e}}\left(\Sigma^{\hat{d} \hat{e}}\right)_{\hat{\alpha} \hat{\beta}} \operatorname{tr}\left(4 \boldsymbol{W}^{2} \boldsymbol{X}^{i j}-5 \mathrm{i} \boldsymbol{W} \boldsymbol{\lambda}^{\hat{\gamma}(i} \boldsymbol{\lambda}_{\hat{\gamma}}^{j)}\right) \\
& +6\left(\Sigma_{[\hat{a} \hat{b}}\right)_{\hat{\alpha} \hat{\beta}}\left(\Gamma_{\hat{c}]}\right)^{\hat{\gamma} \hat{\delta}} \operatorname{tr}\left(\boldsymbol{\lambda}_{\hat{\gamma}}^{(i} \boldsymbol{\lambda}_{\hat{\delta}}^{j)} \boldsymbol{W}\right)+3 \varepsilon^{i j}\left(\Gamma_{[\hat{a}}\right)_{\hat{\alpha} \hat{\beta}}\left(\Sigma_{\hat{b} \hat{c}]}\right)^{\hat{\gamma} \hat{\delta}} \operatorname{tr}\left(\boldsymbol{\lambda}_{\hat{\gamma}}^{k} \boldsymbol{\lambda}_{\hat{\delta} k} \boldsymbol{W}\right) \\
& -6 \mathrm{i} \varepsilon^{i j}\left(\Gamma_{[\hat{a}}\right)_{\hat{\alpha} \hat{\beta}} \operatorname{tr}\left(\boldsymbol{F}_{\hat{b} \hat{c}]} \boldsymbol{W}^{2}\right) \text {, } \\
& \Sigma_{\hat{a} \hat{b} \hat{c} \hat{d} \hat{\alpha}}^{i}=-\frac{1}{2} \varepsilon_{\hat{a} \hat{b} \hat{c} \hat{d} \hat{e}}\left(\Gamma^{\hat{e}}\right){ }_{\hat{\alpha}}^{\hat{\beta}} \operatorname{tr}\left(6 \mathrm{i} W\left\{\boldsymbol{F}_{\hat{\beta} \hat{\gamma}}, \boldsymbol{\lambda}^{\hat{\gamma} i}\right\}-3 \mathrm{i} \boldsymbol{W}\left\{\boldsymbol{X}^{i j}, \boldsymbol{\lambda}_{\hat{\beta} j}\right\}-4 \boldsymbol{\lambda}^{\hat{\gamma}(i} \boldsymbol{\lambda}_{\hat{\gamma}}^{j)} \boldsymbol{\lambda}_{\hat{\beta} j}\right) \\
& +\varepsilon_{\hat{a} \hat{b} \hat{c} \hat{d} \hat{e}}\left(\Gamma^{\hat{e}}\right)^{\hat{\beta} \hat{\gamma}} \operatorname{tr}\left(\boldsymbol{\lambda}_{\hat{\beta}}^{(i} \boldsymbol{\lambda}_{\hat{\gamma}}^{j)} \boldsymbol{\lambda}_{\hat{\alpha} j}\right) \\
& -\frac{\mathrm{i}}{2} \varepsilon_{\hat{a} \hat{b} \hat{c} \hat{d} \hat{e}} \operatorname{tr}\left(\left\{\boldsymbol{W}, \mathcal{D}^{\hat{e}} \boldsymbol{W}\right\} \boldsymbol{\lambda}_{\hat{\alpha}}^{i}\right)-2 \mathrm{i} \varepsilon_{\hat{a} \hat{b} \hat{c} \hat{d} \hat{e}} \operatorname{tr}\left(\boldsymbol{W}^{2} \mathcal{D}^{\hat{e}} \boldsymbol{\lambda}_{\hat{\alpha}}^{i}\right) \\
& -\mathrm{i} \varepsilon_{\hat{a} \hat{b} \hat{c} \hat{d} \hat{e}}\left(\Sigma^{\hat{e} \hat{f}}\right)_{\hat{\alpha}}^{\hat{\beta}} \operatorname{tr}\left(\left\{\boldsymbol{W}, \mathcal{D}_{\hat{f}} \boldsymbol{W}\right\} \boldsymbol{\lambda}_{\hat{\beta}}^{i}\right)-4 \mathrm{i} \varepsilon_{\hat{a} \hat{b} \hat{c} \hat{d} \hat{e}}\left(\Sigma^{\hat{e} \hat{f}}\right)_{\hat{\alpha}}^{\hat{\beta}} \operatorname{tr}\left(\boldsymbol{W}^{2} \boldsymbol{D}_{\hat{f}} \boldsymbol{\lambda}_{\hat{\beta}}^{i}\right) \text {, } \\
& \Sigma_{\hat{a} \hat{b} \hat{c} \hat{d} \hat{e}}=-\frac{3}{2} \varepsilon_{\hat{a} \hat{b} \hat{c} \hat{d} \hat{e}} \operatorname{tr}\left(\boldsymbol{W} \boldsymbol{X}^{k l} \boldsymbol{X}_{k l}-2 \boldsymbol{W} \boldsymbol{F}^{\hat{\gamma} \hat{\delta}} \boldsymbol{F}_{\hat{\gamma} \hat{\delta}}-\mathrm{i} \boldsymbol{X}^{k l} \boldsymbol{\lambda}_{k}^{\hat{\delta}} \boldsymbol{\lambda}_{\hat{\delta} l}-2 \mathrm{i} \boldsymbol{F}^{\hat{\gamma} \hat{\delta}} \boldsymbol{\lambda}_{\hat{\gamma}}^{k} \boldsymbol{\lambda}_{\hat{\delta} k}\right. \\
& +\frac{2}{3} \boldsymbol{W}\left(\mathcal{D}^{\hat{f}} \boldsymbol{W}\right) \mathcal{D}_{\hat{f}} \boldsymbol{W}+\frac{4}{3} \boldsymbol{W}^{2} \mathcal{D}^{\hat{f}} \mathcal{D}_{\hat{f}} \boldsymbol{W}+\mathrm{i} \boldsymbol{W}\left[\mathcal{D}_{\hat{\gamma} \hat{\delta}} \boldsymbol{\lambda}^{\hat{\gamma} k}, \boldsymbol{\lambda}_{k}^{\hat{\delta}}\right] \\
& \left.-2 \boldsymbol{W}^{2} \boldsymbol{\lambda}^{\hat{\gamma} k} \boldsymbol{\lambda}_{\hat{\gamma} k}\right)
\end{aligned}
$$

where

$$
\boldsymbol{\lambda}_{\hat{\alpha}}^{i}:=-\mathrm{i} \mathcal{D}_{\hat{\alpha}}^{i} \boldsymbol{W}, \quad \boldsymbol{X}^{i j}:=-\frac{1}{4} \mathcal{D}^{\hat{\alpha}(i} \boldsymbol{\lambda}_{\hat{\alpha}}^{j)}, \quad \boldsymbol{F}_{\hat{\alpha} \hat{\beta}}=\frac{1}{4} \mathcal{D}_{(\hat{\alpha}}^{k} \boldsymbol{\lambda}_{\hat{\beta}) k} .
$$

The above curvature induced form agrees with the one found in [76] up to the addition of an exact five-form

$$
\Sigma_{\text {exact }}=\mathrm{d} N
$$

with

$$
\begin{aligned}
& N_{\hat{\alpha}}^{i j k l} \hat{\beta} \hat{\gamma}=0, \\
& N_{\hat{a}}^{j k} \hat{\beta}_{\hat{\gamma}}^{j \hat{\gamma}}=0, \quad N_{\hat{a} \hat{b} \hat{\gamma} \hat{\delta}}^{k l}=0, \\
& N_{\hat{a} \hat{b} \hat{c} \hat{c}}^{l}=-\mathrm{i} \varepsilon_{\hat{a} \hat{b} \hat{c} \hat{d} \hat{e}}\left(\Sigma^{\hat{d} \hat{e}}\right)_{\hat{\delta}} \hat{\gamma}_{\operatorname{tr}}\left(\boldsymbol{W}^{2} \boldsymbol{\lambda}_{\hat{\gamma}}^{l}\right) \text {, } \\
& N_{\hat{a} \hat{b} \hat{c} \hat{d}}=0 \text {. }
\end{aligned}
$$

Ignoring boundary terms, the exact form does not change the corresponding action.

It is worth noting that although we can add a total derivative constructed out of $\boldsymbol{W}$ and its covariant derivatives, the curvature induced form is uniquely fixed in conformal supergravity. In particular, a primary generalization of (10.15) in supergravity does not exist. 


\subsection{The non-abelian Chern-Simons action}

Making use of the superform $\Sigma$ one can construct a closed five-form in $5 \mathrm{D}$ from which one can derive a supersymmetric action. We now make use of the closed form,

$$
\mathfrak{J}:=\Sigma_{\mathrm{CS}}-\Sigma_{R}
$$

and the action principle (9.16) together with the formula (8.27). We find the action ${ }^{26}$

$$
\begin{aligned}
& S=\int \mathrm{d}^{5} x e \operatorname{tr}\left\{-\frac{1}{24} \varepsilon^{\hat{a} \hat{b} \hat{c} d \hat{e}} \boldsymbol{v}_{\hat{a}} \boldsymbol{f}_{\hat{b} \hat{c}} \boldsymbol{f}_{\hat{d} \hat{e}}-\frac{\mathrm{i}}{24} \varepsilon^{\hat{a} \hat{b} \hat{c} \hat{d} \hat{e}} \boldsymbol{v}_{\hat{a}} \boldsymbol{v}_{\hat{b}} \boldsymbol{v}_{\hat{c}} \boldsymbol{f}_{\hat{d} \hat{e}}-\frac{1}{60} \varepsilon^{\hat{a} \hat{b} \hat{c} d \hat{e}} \boldsymbol{v}_{\hat{a}} \boldsymbol{v}_{\hat{b}} \boldsymbol{v}_{\hat{c}} \boldsymbol{v}_{\hat{d}} \boldsymbol{v}_{\hat{e}}\right. \\
& -\frac{1}{4} \boldsymbol{W} \boldsymbol{F}_{\hat{a} \hat{b}} \boldsymbol{F}^{\hat{a} \hat{b}}+\frac{1}{4} \boldsymbol{W} \boldsymbol{X}^{i j} \boldsymbol{X}_{i j}+\frac{\mathrm{i}}{4} \boldsymbol{F}_{\hat{a} \hat{b}}\left(\boldsymbol{\lambda}^{k} \sum^{\hat{a} \hat{b}} \boldsymbol{\lambda}_{k}\right)-\frac{\mathrm{i}}{4} \boldsymbol{X}_{i j}\left(\boldsymbol{\lambda}^{i} \boldsymbol{\lambda}^{j}\right) \\
& +\frac{\mathrm{i}}{4} \boldsymbol{W}\left(\boldsymbol{\lambda}^{k} \overleftrightarrow{\nabla} \boldsymbol{\lambda}_{k}\right)+\frac{1}{6} \boldsymbol{W}\left(\boldsymbol{\nabla}^{\hat{a}} \boldsymbol{W}\right) \boldsymbol{\nabla}_{\hat{a}} \boldsymbol{W}+\frac{1}{3} \boldsymbol{W}^{2} \boldsymbol{\nabla}^{\hat{a}} \boldsymbol{\nabla}_{\hat{a}} \boldsymbol{W}-\frac{32}{9} D \boldsymbol{W}^{3} \\
& +\frac{32 \mathrm{i}}{9} \chi^{k} \boldsymbol{\lambda}_{k} \boldsymbol{W}^{2}-\frac{3}{4} W^{\hat{a} \hat{a}} \boldsymbol{F}_{\hat{a} \hat{b}} \boldsymbol{W}^{2}-\frac{5}{12} W^{\hat{a} \hat{a}} W_{\hat{a} \hat{b}} \boldsymbol{W}^{3}-\frac{1}{2} \boldsymbol{W}^{2} \boldsymbol{\lambda}^{k} \boldsymbol{\lambda}_{k} \\
& -\frac{\mathrm{i}}{8}\left(\psi_{\hat{a} i} \Gamma^{\hat{a}} \Sigma^{\hat{b} \hat{c}} \boldsymbol{\lambda}^{i}\right)\left(\left\{\boldsymbol{F}_{\hat{b} \hat{c}}, \boldsymbol{W}\right\}+\boldsymbol{W}^{2} W_{\hat{b} \hat{c}}\right)-\frac{\mathrm{i}}{24} \psi_{\hat{a} i} \Gamma^{\hat{a}} \Gamma^{\hat{b}} \boldsymbol{\lambda}^{i}\left\{\boldsymbol{W}, \boldsymbol{\nabla}_{\hat{b}} \boldsymbol{W}\right\} \\
& -\left(\psi_{\hat{a} i} \Gamma^{\hat{a}} \boldsymbol{\lambda}_{j}\right)\left(\frac{\mathrm{i}}{8}\left\{\boldsymbol{X}^{i j}, \boldsymbol{W}\right\}+\frac{1}{6} \boldsymbol{\lambda}^{(i} \boldsymbol{\lambda}^{j)}\right)+\frac{8 \mathrm{i}}{9}\left(\psi_{\hat{a} i} \Gamma^{\hat{a}} \chi^{i}\right) \boldsymbol{W}^{3} \\
& -\frac{\mathrm{i}}{6} \psi_{\hat{a} i} \Gamma^{\hat{a}} \not \boldsymbol{\lambda}^{i} \boldsymbol{W}^{2}-\frac{1}{12}\left(\psi_{\hat{a} i} \boldsymbol{\lambda}_{j}\right)\left(\boldsymbol{\lambda}^{(i} \Gamma^{\hat{a}} \boldsymbol{\lambda}^{j)}\right)+\frac{\mathrm{i}}{8}\left(\psi_{\hat{c} i} \Sigma^{\hat{a} \hat{b}} \Gamma^{\hat{c}} \boldsymbol{\lambda}^{i}\right) \boldsymbol{W}^{2} W_{\hat{a} \hat{b}} \\
& +\frac{\mathrm{i}}{24}\left(\psi_{\hat{a} k} \psi_{\hat{b}}^{k}\right)\left(2 \boldsymbol{W}^{2} \boldsymbol{F}^{\hat{a} \hat{b}}-3 \mathrm{i} \boldsymbol{\lambda}^{k} \sum^{\hat{a} \hat{b}} \boldsymbol{\lambda}_{k} \boldsymbol{W}+3 W^{\hat{a} \hat{b}} \boldsymbol{W}^{3}\right) \\
& +\frac{\mathrm{i}}{48} \varepsilon^{\hat{a} \hat{b} \hat{c} \hat{d} \hat{e}}\left(\psi_{\hat{a} k} \Gamma_{\hat{b}} \psi_{\hat{c}}^{k}\right) \boldsymbol{F}_{\hat{d} \hat{e}} \boldsymbol{W}^{2}+\frac{\mathrm{i}}{12}\left(\psi_{\hat{a} i} \Sigma^{\hat{a} \hat{b}} \psi_{\hat{b} j}\right)\left(2 \boldsymbol{W}^{2} \boldsymbol{X}^{i j}-3 \mathrm{i} \boldsymbol{W} \boldsymbol{\lambda}^{(i} \boldsymbol{\lambda}^{j)}\right) \\
& +\frac{1}{12}\left(\psi_{\hat{a} i} \Sigma^{\hat{a} \hat{b}} \boldsymbol{\lambda}^{i}\right)\left\{\boldsymbol{W}, \psi_{\hat{b} j} \boldsymbol{\lambda}^{j}\right\}+\frac{1}{16} \varepsilon^{\hat{a} \hat{b} \hat{c} \hat{d} \hat{e}}\left(\psi_{\hat{a} k} \psi_{\hat{b}}^{k}\right)\left(\psi_{\hat{c} j} \Sigma_{\hat{d} \hat{e}} \boldsymbol{\lambda}^{j}\right) \boldsymbol{W}^{2} \\
& \left.+\frac{1}{24} \varepsilon^{\hat{a} \hat{b} \hat{c} \hat{d} \hat{e}}\left(\psi_{\hat{a} j} \Sigma_{\hat{b} \hat{c}} \psi_{\hat{d}^{k}}\right)\left(\psi_{\hat{e}}^{j} \boldsymbol{\lambda}^{k}\right) \boldsymbol{W}^{2}+\frac{1}{96} \varepsilon^{\hat{a} \hat{b} \hat{c} \hat{d} \hat{e}}\left(\psi_{\hat{a} k} \Gamma_{\hat{b}} \psi_{\hat{c}}^{k}\right)\left(\psi_{\hat{d}^{j}} \psi_{\hat{e}^{j}}^{j}\right) \boldsymbol{W}^{3}\right\},
\end{aligned}
$$

where we have defined

$$
\frac{\mathrm{i}}{2}\left[\boldsymbol{\nabla}_{\hat{\gamma} \hat{\delta}} \boldsymbol{\lambda}^{\hat{\gamma} k}, \boldsymbol{\lambda}_{k}^{\hat{\delta}}\right]=\frac{\mathrm{i}}{2} \boldsymbol{\lambda}^{k} \overleftrightarrow{\nabla} \boldsymbol{\lambda}_{k}:=\frac{\mathrm{i}}{2} \boldsymbol{\lambda}^{k} \boldsymbol{\nabla} \boldsymbol{\lambda}_{k}-\frac{\mathrm{i}}{2}\left(\boldsymbol{\nabla} \boldsymbol{\lambda}^{k}\right) \boldsymbol{\lambda}_{k}
$$

and introduced the bar-projected field strength and one-form:

$$
\boldsymbol{f}_{\hat{a} \hat{b}}=2 e_{\hat{a}}^{\hat{m}} e_{\hat{b}}^{\hat{n}}\left(\partial_{[\hat{m}} \boldsymbol{v}_{\hat{n}]}-\mathrm{i} \boldsymbol{v}_{[\hat{m}} \boldsymbol{v}_{\hat{n}]}\right), \quad \boldsymbol{v}_{\hat{a}}:=e_{\hat{a}}^{\hat{m}} \boldsymbol{v}_{\hat{m}}, \quad \boldsymbol{v}_{\hat{m}}:=\boldsymbol{V}_{\hat{m}} \mid .
$$

The vector covariant derivatives of the component fields may be expressed in terms of the $\hat{\mathcal{D}}_{\hat{a}}$ derivatives and hatted component fields introduced in section 4 . For completeness we include the following component results:

$$
\begin{aligned}
& \boldsymbol{\nabla}_{\hat{a}} \boldsymbol{W}=\hat{\mathcal{D}}_{\hat{a}} \boldsymbol{W}-\frac{\mathrm{i}}{2} \psi_{\hat{a}}^{\hat{\gamma}} \boldsymbol{\lambda}_{\hat{\gamma}}^{k}, \\
& \boldsymbol{\nabla}^{\hat{a}} \boldsymbol{\nabla}_{\hat{a}} \boldsymbol{W}=\hat{\mathcal{D}}^{\hat{a}} \nabla_{\hat{a}} \boldsymbol{W}-\frac{\mathrm{i}}{2} \psi^{\hat{a} \hat{\beta}}{ }_{j}^{\hat{a}} \boldsymbol{\nabla}_{\hat{a}} \boldsymbol{\lambda}_{\hat{\beta}}^{j}+\frac{\mathrm{i}}{2} \psi_{\hat{\beta}}{ }_{j}^{\hat{\gamma}}{ }_{j}^{\hat{\beta}} W_{\hat{\gamma} \hat{\delta}} \boldsymbol{\lambda}^{\hat{\delta} j}-\frac{8 \mathrm{i}}{3} \psi_{\hat{\beta}}{ }_{j}^{\hat{\gamma}}{ }_{j}^{\hat{\beta}} \chi_{\hat{\gamma}}^{j} \boldsymbol{W}
\end{aligned}
$$

\footnotetext{
${ }^{26}$ Here we understand the superfield $\boldsymbol{W}$ and the superfields constructed from its covariant derivatives as their corresponding component fields. It should be clear from context which we are referring to.
} 


$$
\begin{aligned}
& +\frac{i}{2} \psi_{\hat{\beta}}^{\hat{\gamma} \hat{\beta}}{ }_{j}\left[\boldsymbol{\lambda}_{\hat{\gamma}}^{j}, \boldsymbol{W}\right]-2 \hat{\mathfrak{f}}_{\hat{a}}^{\hat{a}} \boldsymbol{W}+\frac{1}{2} \hat{\phi}_{\hat{\beta}}^{\hat{\gamma}}{ }^{\hat{\beta} j} \boldsymbol{\lambda}_{\hat{\gamma} j} \\
& +\frac{20 \mathrm{i}}{3} \chi^{\hat{\alpha} i} \boldsymbol{\lambda}_{\hat{\alpha} i}+\frac{20}{3} D \boldsymbol{W}+\frac{1}{32} W^{\hat{a} \hat{b}} W_{\hat{a} \hat{b}} \boldsymbol{W} \\
\boldsymbol{\nabla}_{\hat{a}} \boldsymbol{\lambda}_{\hat{\alpha}}^{i}= & \hat{\mathcal{D}}_{\hat{a}} \boldsymbol{\lambda}_{\hat{\alpha}}^{i}-\psi_{\hat{a}}^{\hat{\beta} i}\left(\boldsymbol{F}_{\hat{\beta} \hat{\alpha}}+W_{\hat{\beta} \hat{\alpha}} \boldsymbol{W}\right)-\frac{1}{2} \psi_{\hat{a} \hat{\alpha} j} \boldsymbol{X}^{i j}-\frac{1}{2} \psi_{\hat{a}}^{\hat{\beta} i} \boldsymbol{\nabla}_{\hat{\beta} \hat{\alpha}} \boldsymbol{W} \\
& -\mathrm{i} \hat{\phi}_{\hat{a} \hat{\alpha}}^{i} \boldsymbol{W}+\frac{1}{4} \tilde{W}_{\hat{a} \hat{b} \hat{c}}\left(\Sigma^{\hat{b} \hat{c}}\right)_{\hat{\alpha}}^{\hat{\beta}} \boldsymbol{\lambda}_{\hat{\beta}}^{i}-\frac{8}{3}\left(\Gamma_{\hat{a}}\right)_{\hat{\alpha}}^{\hat{\beta}} \chi_{\hat{\beta}}^{i} \boldsymbol{W},
\end{aligned}
$$

where

$$
\hat{\mathcal{D}}_{\hat{a}}=\hat{\mathcal{D}}_{\hat{a}}-\mathrm{i} \boldsymbol{V}_{\hat{a}} .
$$

Note that the covariant field strength may be expressed in terms of the bar-projected field strength. Performing the component projection of the identity

$$
\boldsymbol{F}_{\hat{m} \hat{n}}=E_{\hat{m}}^{\hat{A}} E_{\hat{n}}^{\hat{B}} \boldsymbol{F}_{\hat{A} \hat{B}}(-1)^{\varepsilon} \hat{A}^{\varepsilon} \hat{B},
$$

we find

$$
\boldsymbol{F}_{\hat{a} \hat{b}} \mid=\boldsymbol{f}_{\hat{a} \hat{b}}+\mathrm{i}\left(\Gamma_{[\hat{a}}\right)_{\hat{\alpha}}^{\hat{\beta}} \psi_{\hat{b}] k}^{\hat{\alpha}} \boldsymbol{\lambda}_{\hat{\beta}}^{k}+\frac{\mathrm{i}}{2} \psi_{[\hat{a} k}^{\hat{\gamma}} \psi_{\hat{b}] \hat{\gamma}}^{k} \boldsymbol{W}
$$

It should be mentioned that the abelian Chern-Simons action can straightforwardly be read off of the action presented in this section.

\section{Supermultiplets with gauged central charge}

In the presence of a gauged central charge different off-shell multiplets in conformal supergravity become possible. For example, in $4 \mathrm{D} \mathcal{N}=2$ conformal supergravity there exist so-called vector-tensor multiplets, which may be viewed as dual versions of the abelian vector multiplet and possess gauge two-forms. ${ }^{27}$ The situation in $5 \mathrm{D}$ conformal supergravity is similar. There also exists a dual version of the abelian vector multiplet, which we refer to as the two-form multiplet. The off-shell multiplet was first constructed in [20] within the component approach and was shown that it may be generalized to a so-called large tensor multiplet that may be given a mass. Recently, two of us have shown how to describe both the two-form and large tensor multiplets in Minkowski superspace by making convenient use of superform formulations [77].

In this section we generalize the results of [77] to conformal superspace. Firstly, we discuss how to gauge the central charge in conformal superspace. We then give the superform formulation for the linear multiplet with central charge and immediately derive its corresponding action principle. The action provides an important ingredient in constructing actions for multiplets with gauged central charge. Finally, we give the superform formulations for the gauge two-form and large tensor multiplets.

\footnotetext{
${ }^{27}$ See $[79,80]$ for a superspace description of all known off-shell vector-tensor multiplets in $4 \mathrm{D} \mathcal{N}=2$ conformal supergravity.
} 


\subsection{Gauging a central charge in conformal superspace}

We can introduce a central charge $\Delta$ in conformal superspace and gauge it using an abelian vector multiplet associated with a gauge connection $\mathcal{V}$. Doing so requires that we follow a similar procedure as the one used in section 2.6. We can obtain the resulting structure by simply replacing the gauge connection $V$ and field strength $F$ with those associated with the central charge $\Delta$ as follows:

$$
\mathrm{i} V \rightarrow \mathcal{V} \Delta, \quad \mathrm{i} F \rightarrow \mathcal{F} \Delta .
$$

The central charge is required to commute with the covariant derivatives

$$
\left[\Delta, \nabla_{\hat{A}}\right]=0
$$

and annihilate both $\mathcal{V}$ and $\mathcal{F}$

$$
\Delta \mathcal{V}=0, \quad \Delta \mathcal{F}=0
$$

The central charge gauge transformations of the covariant derivatives are

$$
\delta \nabla_{\hat{A}}=\left[\Lambda \Delta, \nabla_{\hat{A}}\right] \Longrightarrow \delta \mathcal{V}_{\hat{A}}=\nabla_{\hat{A}} \Lambda
$$

where the gauge parameter $\Lambda$ is inert under the central charge, $\Delta \Lambda=0$.

We constrain the field strength $\mathcal{F}$ formally the same way as $F$ but with $W$ replaced by $\mathcal{W}$. The components of $\mathcal{F}$ are given by the following:

$$
\begin{aligned}
\mathcal{F}_{\hat{\alpha} \hat{\beta}}^{i j} & =2 \mathrm{i} \varepsilon^{i j} \varepsilon_{\hat{\alpha} \hat{\beta}} \mathcal{W}, \\
\mathcal{F}_{\hat{a} \hat{\beta}}^{j} & =-\left(\Gamma_{\hat{a}}\right)_{\hat{\beta}}^{\hat{\gamma}} \nabla_{\hat{\gamma}}^{j} \mathcal{W}, \\
\mathcal{F}_{\hat{a} \hat{b}} & =-\frac{\mathrm{i}}{4}\left(\Sigma_{\hat{a} \hat{b}}\right)^{\hat{\alpha} \hat{\beta}}\left(\nabla_{\hat{\alpha}}^{k} \nabla_{\hat{\beta} k}-4 \mathrm{i} W_{\hat{\alpha} \hat{\beta}}\right) \mathcal{W},
\end{aligned}
$$

with $\mathcal{W}$ constrained by the Bianchi identity

$$
\nabla_{\hat{\alpha}}^{(i} \nabla_{\hat{\beta}}^{j)} \mathcal{W}=\frac{1}{4} \varepsilon_{\hat{\alpha} \hat{\beta}} \nabla^{\hat{\gamma}(i} \nabla_{\hat{\gamma}}^{j)} \mathcal{W}
$$

The above results will be used in the remainder of this section.

\subsection{The linear multiplet with central charge}

In this subsection we construct a superform formulation for the 5D linear multiplet with gauged central charge in conformal superspace, generalizing the one given in [77]. Our approach is similar to the one adopted for the $4 \mathrm{D} \mathcal{N}=2$ linear multiplet in conformal supergravity [70]. We will show that the superform formulation naturally leads to the action principle based on a linear multiplet. 


\subsubsection{Superform formulation for the linear multiplet}

In [70] a superform formulation for the $4 \mathrm{D} \mathcal{N}=2$ linear multiplet was found by extending the vielbein to include the one-form gauging the central charge. This leads to a system of superforms describing the linear multiplet. As in [77] we instead start with a system of superforms that generalizes the one that appeared in [70].

We introduce two primary superforms: a five-form $\tilde{\Sigma}$ and a four-form $\Phi$. We require that they satisfy the superform equations

$$
\nabla \tilde{\Sigma}=F \wedge \Phi, \quad \nabla \Phi=-\Delta \tilde{\Sigma}
$$

and transform as scalars under the gauge transformations (11.4)

$$
\delta \tilde{\Sigma}=\Lambda \Delta \tilde{\Sigma}, \quad \delta \Phi=\Lambda \Delta \Phi .
$$

The superforms $\tilde{\Sigma}$ and $\Phi$ can now be related to the linear multiplet with central charge by imposing certain constraints. However, it will prove useful to first introduce some notation to deal with the component form of (11.7).

We introduce indices that range over not just $\hat{A}$ but an additional bosonic coordinate, $\hat{\mathcal{A}}=(\hat{A}, 6)$. Then we may rewrite eq. (11.7) in components as

$$
\nabla_{[\hat{\mathcal{A}}} \Sigma_{\hat{\mathcal{B}} \hat{\mathcal{D}} \hat{\mathcal{E}} \hat{\mathcal{F}}\}}+\frac{5}{2} \mathscr{T}_{[\hat{\mathcal{A}} \hat{\mathcal{B}}}^{\hat{\mathcal{G}}} \Sigma_{|\hat{\mathcal{G}}| \hat{\mathcal{C}} \hat{\mathcal{D}} \hat{\mathcal{F}} \hat{\mathcal{F}}\}}=0
$$

where we have made the identifications

$$
\mathscr{T}_{\hat{A} \hat{B}}{ }^{6}=F_{\hat{A} \hat{B}}, \quad \mathscr{T}_{6 \hat{B}} \hat{\mathcal{A}}^{\hat{\mathcal{B}}}=\mathscr{T}_{\hat{B} 6}{ }^{\hat{\mathcal{A}}}=0, \quad \nabla_{6}=\Delta
$$

and

$$
\begin{aligned}
& \tilde{\Sigma}=\frac{1}{5 !} E^{\hat{E}} \wedge E^{\hat{D}} \wedge E^{\hat{C}} \wedge E^{\hat{B}} \wedge E^{\hat{A}} \Sigma_{\hat{A} \hat{B} \hat{C} \hat{D} \hat{E}}, \\
& \Phi=\frac{1}{4 !} E^{\hat{D}} \wedge E^{\hat{C}} \wedge E^{\hat{B}} \wedge E^{\hat{A}} \Sigma_{6 \hat{A} \hat{B} \hat{C} \hat{D}} .
\end{aligned}
$$

We constrain the lowest dimension components by

$$
\begin{aligned}
& \sum_{\hat{\alpha} \hat{\beta} \hat{\gamma} \hat{\delta} \hat{\rho}}^{i j k l p}=\Sigma_{\hat{a} \hat{\alpha} \hat{\beta} \hat{\gamma} \hat{\delta}}^{i j k l}=\Sigma_{\hat{a} \hat{b} \hat{\alpha} \hat{\beta} \hat{\gamma}}^{i j k}=\Sigma_{6 \hat{\alpha} \hat{\beta} \hat{\gamma} \hat{\delta}}^{i j k l}=\Sigma_{6 \hat{a}_{\hat{\beta}} \hat{\gamma} \hat{\gamma} \hat{\delta}}^{j k l}=0, \\
& \Sigma_{6 \hat{a} \hat{b} \hat{\alpha} \hat{\beta}}=8 \mathrm{i}\left(\Sigma_{\hat{a} \hat{b}}\right)_{\hat{\alpha} \hat{\beta}} \mathcal{L}^{i j},
\end{aligned}
$$

and analyze eq. (11.9). The remaining components are completely determined as follows:

$$
\begin{aligned}
\Sigma_{\hat{a} \hat{b} \hat{c} \hat{\alpha}}^{i j} \hat{\beta} & =4 \mathrm{i} \varepsilon_{\hat{a} \hat{b} \hat{c} \hat{d} \hat{e}}\left(\Sigma^{\hat{d} \hat{e}}\right)_{\hat{\alpha} \hat{\beta}} \mathcal{W} \mathcal{L}^{i j}, \\
\Sigma_{6 \hat{a} \hat{b} \hat{c} \hat{\alpha}}^{i} & =-\frac{2}{3} \varepsilon_{\hat{a} \hat{b} \hat{c} \hat{d} \hat{e}}\left(\Sigma^{\hat{d} \hat{e}}\right)_{\hat{\alpha}}^{\hat{\beta}} \nabla_{\hat{\beta} j} \mathcal{L}^{j i}=-2 \varepsilon_{\hat{a} \hat{b} \hat{c} \hat{d} \hat{e}}\left(\Sigma^{\hat{d} \hat{e}}\right)_{\hat{\alpha}}{ }^{\hat{\beta}} \varphi_{\hat{\beta}}^{i}, \\
\Sigma_{\hat{a} \hat{b} \hat{c} \hat{d} \hat{\alpha}} & =-2 \varepsilon_{\hat{a} \hat{b} \hat{c} \hat{d} \hat{e}}\left(\Gamma^{\hat{e}}\right)_{\hat{\alpha}}^{\hat{\beta}}\left(\mathcal{W} \varphi_{\hat{\beta}}^{i}+\mathrm{i} \lambda_{\hat{\beta} j} \mathcal{L}^{j i}\right), \\
\Sigma_{6 \hat{a} \hat{b} \hat{c} \hat{d}} & =\frac{\mathrm{i}}{12} \varepsilon_{\hat{a} \hat{b} \hat{b} \hat{d} \hat{e} \hat{e}}\left(\Gamma^{\hat{e}}\right)^{\hat{\alpha} \hat{\beta}} \nabla_{\hat{\alpha}}^{i} \nabla_{\hat{\beta}}^{j} \mathcal{L}_{i j} \equiv \varepsilon_{\hat{a} \hat{b} \hat{c} \hat{d} \hat{e}} \Phi^{\hat{e}}, \\
\Sigma_{\hat{a} \hat{b} \hat{b} \hat{d} \hat{e}} & =\varepsilon_{\hat{a} \hat{b} \hat{c} \hat{d} \hat{e}}\left(\mathcal{W} F+X^{i j} \mathcal{L}_{i j}+2 \lambda^{\hat{\gamma} k} \varphi_{\hat{\gamma} k}\right),
\end{aligned}
$$


where $\mathcal{L}^{i j}$ satisfies the constraint for the linear multiplet

$$
\nabla_{\hat{\alpha}}^{(i} \mathcal{L}^{j k)}=0
$$

and we have introduced the superfields

$$
\begin{aligned}
\varphi_{\hat{\alpha}}^{i} & :=\frac{1}{3} \nabla_{\hat{\alpha} j} \mathcal{L}^{i j} \\
F & :=\frac{\mathrm{i}}{12} \boldsymbol{\nabla}^{\hat{\gamma} i} \nabla_{\hat{\gamma}}^{j} \mathcal{L}_{i j}=-\frac{\mathrm{i}}{4} \boldsymbol{\nabla}^{\hat{\gamma} k} \varphi_{\hat{\gamma} k} .
\end{aligned}
$$

The above superfields together with

$$
\Phi_{\hat{a}}=\frac{\mathrm{i}}{12}\left(\Gamma_{\hat{a}}\right)^{\hat{\alpha} \hat{\beta}} \nabla_{\hat{\alpha}}^{i} \nabla_{\hat{\beta}}^{j} \mathcal{L}_{i j}=-\frac{\mathrm{i}}{4}\left(\Gamma_{\hat{a}}\right)^{\hat{\alpha} \hat{\beta}} \nabla_{\hat{\alpha}}^{k} \varphi_{\hat{\beta} k}
$$

satisfy the following useful identities:

$$
\begin{aligned}
\nabla_{\hat{\alpha}}^{i} \mathcal{L}^{j k}= & 2 \varepsilon^{i(j} \varphi_{\hat{\alpha}}^{k)}, \\
\nabla_{\hat{\alpha}}^{i} \varphi_{\hat{\beta}}^{j}= & -\frac{\mathrm{i}}{2} \varepsilon^{i j} \varepsilon_{\hat{\alpha} \hat{\beta}} F+\frac{\mathrm{i}}{2} \varepsilon^{i j} \Phi_{\hat{\alpha} \hat{\beta}}+\mathrm{i} \nabla_{\hat{\alpha} \hat{\beta}} \mathcal{L}^{i j}+\mathrm{i} \varepsilon_{\hat{\alpha} \hat{\beta}} W \Delta \mathcal{L}^{i j}, \\
\nabla_{\hat{\alpha}}^{i} F= & -2 \nabla_{\hat{\alpha}}^{\hat{\beta}} \varphi_{\hat{\beta}}^{i}-2 \mathrm{i} \lambda_{\hat{\alpha} j} \Delta \mathcal{L}^{i j}-6 W_{\hat{\alpha} \hat{\beta}} \varphi^{\hat{\beta} i}-9 X_{\hat{\alpha} j} \mathcal{L}^{i j} \\
\nabla_{\hat{\alpha}}^{i} \Phi_{\hat{a}}= & 4\left(\Sigma_{\hat{a} \hat{b}}\right)_{\hat{\alpha}}^{\hat{\beta}} \nabla^{\hat{b}} \varphi_{\hat{\beta}}^{i}-2 \mathrm{i}\left(\Gamma_{\hat{a}}\right)_{\hat{\alpha}}^{\hat{\beta}} \lambda_{\hat{\beta} j} \Delta \mathcal{L}^{i j}-2\left(\Gamma_{\hat{a}}\right)_{\hat{\alpha}}{ }_{\hat{\beta}} W \Delta \varphi_{\hat{\beta}}^{i} \\
& -4\left(\Gamma_{\hat{a}}\right)_{\hat{\alpha}}^{\hat{\beta}} W_{\hat{\beta} \hat{\gamma}} \varphi^{\hat{\gamma} i}-6\left(\Gamma_{\hat{a}}\right)_{\hat{\alpha}}^{\hat{\beta}} X_{\hat{\beta} j} \mathcal{L}^{i j}
\end{aligned}
$$

Using the additional identities

$$
\begin{aligned}
S_{\hat{\alpha}}^{i} \varphi_{\hat{\beta}}^{j} & =-6 \varepsilon_{\hat{\alpha} \hat{\beta}} \mathcal{L}^{i j}, \\
S_{\hat{\alpha}}^{i} F & =6 \mathrm{i} \varphi_{\hat{\alpha}}^{i}, \\
S_{\hat{\alpha}}^{i} \Phi_{\hat{b}} & =-8 \mathrm{i}\left(\Gamma_{\hat{b}}\right)_{\hat{\alpha}}{ }^{\hat{\beta}} \varphi_{\hat{\beta}}^{i},
\end{aligned}
$$

one can check that $\tilde{\Sigma}$ and $\Phi$ are primary.

The superform equations imply the differential condition on $\Phi_{\hat{a}}$

$$
\nabla^{\hat{a}} \Phi_{\hat{a}}=\Delta\left(\mathcal{W} F+X^{i j} \mathcal{L}_{i j}+2 \Lambda^{\hat{\gamma} k} \varphi_{\hat{\gamma} k}\right)-5 \mathrm{i} X^{\hat{\gamma} k} \varphi_{\hat{\gamma} k}
$$

It should be mentioned that in the above the central charge transformation of $\mathcal{L}^{i j}$ is arbitrary. If we instead require $\mathcal{L}^{i j}$ to be inert under the central charge, $\Delta \mathcal{L}^{i j}=0$, we have

$$
\mathrm{d} \Phi=0
$$

and $\mathcal{L}^{i j}$ becomes an $\mathcal{O}(2)$ multiplet already described in previous sections. 


\subsubsection{Action principle}

Having derived the components of $\Sigma_{\hat{\mathcal{A}} \hat{\mathcal{C}} \hat{\mathcal{D}} \hat{\mathcal{E}}}$, it is straightforward to construct a closed five-form. It is

$$
\mathfrak{J}=\tilde{\Sigma}+V \wedge \Phi
$$

One can check that it is closed,

$$
\mathrm{d} J=\mathrm{d} \tilde{\Sigma}+V \wedge \mathrm{d} \Phi+\mathrm{d} V \wedge \Phi=\nabla \tilde{\Sigma}+V \wedge \Delta \tilde{\Sigma}+V \wedge \nabla \Phi+F \wedge \Phi=0
$$

and it transforms by an exact form under the central charge transformations,

$$
\begin{aligned}
\delta_{\Lambda} J & =\delta_{\Lambda} \Sigma-\delta_{\Lambda} V \wedge \Phi-V \wedge \delta_{\Lambda} \Phi \\
& =\Lambda \Delta \Sigma-\mathrm{d} \Lambda \wedge \Phi-V \wedge(\Lambda \Delta \Phi)=-\mathrm{d}(\Lambda \Phi) .
\end{aligned}
$$

The corresponding action is found using eq. (8.11) to be

$$
\begin{array}{rl}
S=-\int \mathrm{d}^{5} & x e\left(v^{\hat{a}} \phi_{\hat{a}}+W F+X^{i j} \mathcal{L}_{i j}+2 \lambda^{k} \varphi_{k}\right. \\
& \left.-\psi_{\hat{a} i} \Gamma^{\hat{a}} \varphi^{i} W-\mathrm{i} \psi_{\hat{a} i} \Gamma^{\hat{a}} \lambda_{j} \mathcal{L}^{i j}+\mathrm{i} \psi_{\hat{a} i} \Sigma^{\hat{a} \hat{b}} \psi_{\hat{b} j} W \mathcal{L}^{i j}\right),
\end{array}
$$

where all superfields appearing in the action are understood as their component projections and we have defined

$$
v_{\hat{a}}:=e_{\hat{a}}^{\hat{m}} V_{\hat{m}}\left|, \quad \phi^{\hat{a}}:=-\frac{1}{4 !} e_{\hat{r}}^{\hat{a}} \varepsilon^{\hat{m} \hat{n} \hat{p} \hat{q} \hat{r}} \Phi_{\hat{m} \hat{n} \hat{p} \hat{q}}\right| .
$$

For completeness we also give the component field projection of $\Phi_{\hat{a}}$ :

$$
\Phi^{\hat{a}}\left|=\phi^{\hat{a}}-2 \psi_{\hat{b} k} \Sigma^{\hat{a} \hat{b}} \varphi^{k}+\frac{3 \mathrm{i}}{8} \varepsilon^{\hat{a} \hat{b} \hat{c} \hat{d} \hat{e}} \psi_{\hat{b} k} \Sigma_{\hat{c} \hat{d}} \psi_{\hat{e} l} \mathcal{L}^{k l}\right| .
$$

\subsection{Gauge two-form multiplet}

In superspace, the two-form multiplet is described by a constrained real superfield $L$ that is coupled to the vector multiplet gauging the central charge [33, 77], similar to the $4 \mathrm{D}$ $\mathcal{N}=2$ vector-tensor multiplets. Here we show how a geometric formulation of the multiplet naturally leads to the constraints on $L$ in conformal supergravity. Our presentation is similar to the one given in [77] in Minkowski superspace.

In this subsection we wish to describe couplings of the two-form multiplet to additional Yang-Mills multiplets $\boldsymbol{W}$. Therefore in what follows we make use of covariant derivatives which contain both the gauge connection gauging the central charge and the Yang-Mills gauge connection:

$$
\boldsymbol{\nabla}=\mathrm{d}-\mathcal{V} \Delta-\mathrm{i} \boldsymbol{V}, \quad \nabla_{\hat{A}}=\nabla_{\hat{A}}-\mathcal{V}_{\hat{A}} \Delta-\mathrm{i} \boldsymbol{V}_{\hat{A}} .
$$

We introduce a gauge two-form, $\mathscr{B}=\frac{1}{2} E^{B} E^{A} \mathscr{B}_{A B}$ and define its three-form field strength $\mathscr{H}$ by

$$
\mathscr{H}:=\nabla \mathscr{B}-\operatorname{tr}\left(\boldsymbol{V} \wedge \boldsymbol{F}-\frac{\mathrm{i}}{3} \boldsymbol{V} \wedge \boldsymbol{V} \wedge \boldsymbol{V}\right),
$$


where $\boldsymbol{V}$ and $\boldsymbol{F}$ are the Yang-Mills connection and field strength corresponding to the superfield $\boldsymbol{W} .{ }^{28}$ Here $\mathscr{B}$ is a gauge singlet but is not assumed to be annihilated by the central charge. The (infinitesimal) transformation law for the system of superforms is

$$
\begin{aligned}
\delta \mathcal{V} & =\mathrm{d} \Lambda, & \Delta \Lambda & =0, \\
\delta \boldsymbol{V} & =\mathrm{d} \boldsymbol{\tau}-\mathrm{i}[\boldsymbol{V}, \boldsymbol{\tau}], & \Delta \tau & =0, \\
\delta \mathscr{B} & =\Lambda \Delta \mathscr{B}-\operatorname{tr}(\tau \wedge \mathrm{d} V)+\mathrm{d} \Xi, & \Delta \Xi & =0,
\end{aligned}
$$

where $\Lambda, \tau$ and $\Xi$ generate the gauge transformations of $\mathcal{V}, \boldsymbol{V}$ and $\mathscr{B}$, respectively. The field strength $\mathscr{H}$ transforms covariantly under the central charge transformations

$$
\delta \mathscr{H}=\Lambda \Delta \mathscr{H}
$$

and satisfies the Bianchi identity

$$
\nabla \mathscr{H}=-\mathcal{F} \wedge \Delta \mathscr{B}-\operatorname{tr}(\boldsymbol{F} \wedge \boldsymbol{F}) .
$$

Again we can make use of the notation that was introduced in section 11.2.1. We extend the Bianchi identity by introducing an additional bosonic index, $\hat{\mathcal{A}}=(\hat{A}, 6)$. This can be done because we also have the additional superform equation

$$
\Delta \mathscr{H}=\nabla(\Delta \mathscr{B})
$$

Combining the above equation with the Bianchi identity (11.31) gives

$$
\nabla_{[\hat{\mathcal{A}}} \mathscr{H}_{\hat{\mathcal{B}} \hat{\mathcal{D}}\}}+\frac{3}{2} \mathscr{T}_{[\hat{\mathcal{A}} \hat{\mathcal{B}}} \hat{\mathcal{H}}_{|\hat{\mathcal{E}}| \hat{\mathcal{C}} \hat{\mathcal{D}}\}}+\frac{3}{2} \operatorname{tr}\left(\boldsymbol{F}_{[\hat{\mathcal{A}} \hat{\mathcal{B}}} \boldsymbol{F}_{\hat{\mathcal{C}} \hat{\mathcal{D}}\}}\right)=0
$$

where we have defined

$$
\begin{array}{rlrl}
\mathscr{H}_{6 \hat{A} \hat{B}}:=\Delta \mathscr{B}_{\hat{A} \hat{B}}, & \boldsymbol{F}_{6 \hat{\mathcal{A}}}=\boldsymbol{F}_{\hat{\mathcal{A}} 6}=0, \\
\mathscr{T}_{\hat{A} \hat{B}}{ }^{6}:=\mathcal{F}_{\hat{A} \hat{B}}, & \mathscr{T}_{\hat{A} 6}^{\hat{\mathcal{B}}}=\mathscr{T}_{6 \hat{A}}{ }^{\hat{\mathcal{B}}}=0, & \mathcal{D}_{6}:=\Delta .
\end{array}
$$

Constraining the lowest components of $\mathscr{H}_{\hat{\mathcal{A}} \hat{\mathcal{B}} \hat{\mathcal{C}}}$ by

$$
\mathscr{H}_{\hat{\alpha}}^{i j k} \hat{\beta} \hat{\gamma}=0, \quad \mathscr{H}_{6}^{i j} \hat{\alpha}_{\hat{\beta}}=-2 \mathrm{i} \varepsilon^{i j} \varepsilon_{\hat{\alpha} \hat{\beta}} L
$$

fixes the remaining components of $\mathscr{H}_{\hat{\mathcal{A}} \hat{\mathcal{B}} \hat{\mathcal{C}}}$. Analyzing eq. (11.33) by increasing dimension and subject to the constraints (11.35) (and the identifications (11.34)) leads to the remaining components:

$$
\begin{aligned}
& \mathscr{H}_{\hat{a}}^{j k \hat{\beta}}=-2 \mathrm{i} \varepsilon^{j k}\left(\Gamma_{\hat{a}}\right)_{\hat{\beta} \hat{\gamma}}\left(\mathcal{W} L-\operatorname{tr}\left(\boldsymbol{W}^{2}\right)\right), \\
& \mathscr{H}_{6 \hat{a}_{\hat{\beta}}^{j}}=\left(\Gamma_{\hat{a}}\right)_{\hat{\beta}}^{\hat{\gamma}} \nabla_{\hat{\gamma}}^{j} L
\end{aligned}
$$

\footnotetext{
${ }^{28}$ The special case of $n$ abelian vector multiplets may be obtained by taking $\operatorname{tr}(V \wedge F) \rightarrow \eta_{I J} V^{I} F^{J}$, where $\eta$ is a symmetric, $\eta_{I J}=\eta_{J I}$, coupling constant and $V^{I}$ and $F^{I}$ are the gauge connections and field strengths of the abelian vector multiplets.
} 


$$
\begin{aligned}
\mathscr{H}_{\hat{a} \hat{b} \hat{\gamma}}^{k}= & 2\left(\Sigma_{\hat{a} \hat{b}}\right) \hat{\gamma}{ }^{\hat{\delta}} \nabla_{\hat{\delta}}^{k}\left(\mathcal{W} L-\operatorname{tr}\left(\boldsymbol{W}^{2}\right)\right), \\
\mathscr{H}_{6 \hat{a} \hat{b}}= & \frac{\mathrm{i}}{4}\left(\Sigma_{\hat{a} \hat{b}}\right)^{\hat{\alpha} \hat{\beta}}\left(\nabla_{\hat{\alpha}}^{k} \nabla_{\hat{\beta} k}-4 \mathrm{i} W_{\hat{\alpha} \hat{\beta}}\right) L, \\
\mathscr{H}_{\hat{a} \hat{b} \hat{c}}= & -\frac{\mathrm{i}}{8} \varepsilon_{\hat{a} \hat{b} \hat{c} \hat{d} \hat{e}}\left(\Sigma^{\hat{d} \hat{e}}\right)^{\hat{\alpha} \hat{\beta}}\left(\left(\nabla_{\hat{\alpha}}^{k} \boldsymbol{\nabla}_{\hat{\beta} k}+4 \mathrm{i} W_{\hat{\alpha} \hat{\beta}}\right)\left(\mathcal{W} L-\operatorname{tr}\left(\boldsymbol{W}^{2}\right)\right)\right. \\
& \left.+2\left(\nabla_{\hat{\alpha}}^{k} \mathcal{W}\right) \boldsymbol{\nabla}_{\hat{\beta} k} L-2 \operatorname{tr}\left(\left(\nabla_{\hat{\alpha}}^{k} \boldsymbol{W}\right) \boldsymbol{\nabla}_{\hat{\beta} k} \boldsymbol{W}\right)\right)
\end{aligned}
$$

where $L$ satisfies the constraints

$$
\begin{gathered}
\boldsymbol{\nabla}_{\hat{\alpha}}^{(i} \nabla_{\hat{\beta}}^{j)} L=\frac{1}{4} \varepsilon_{\hat{\alpha} \hat{\beta}} \nabla^{\hat{\gamma}(i} \nabla_{\hat{\gamma}}^{j)} L, \\
\boldsymbol{\nabla}^{\hat{\gamma}(i} \nabla_{\hat{\gamma}}^{j)}\left(\mathcal{W} L-\operatorname{tr}\left(\boldsymbol{W}^{2}\right)\right)=-2\left(\boldsymbol{\nabla}^{\hat{\gamma}(i} \mathcal{W}\right) \nabla_{\hat{\gamma}}^{j)} L+2 \operatorname{tr}\left(\left(\boldsymbol{\nabla}^{\hat{\gamma}(i} \boldsymbol{W}\right) \boldsymbol{\nabla}_{\hat{\gamma}}^{j)} \boldsymbol{W}\right) .
\end{gathered}
$$

To describe the action for the two-form multiplet one can use the composite linear multiplet ${ }^{29}$

$$
\mathcal{L}^{i j}=\frac{\mathrm{i}}{2}\left(2\left(\boldsymbol{\nabla}^{\hat{\alpha}(i} L\right) \nabla_{\hat{\alpha}}^{j)} L+L \nabla^{\hat{\alpha}(i} \nabla_{\hat{\alpha}}^{j)} L\right)=\frac{\mathrm{i}}{6 L} \nabla^{i j}\left(L^{3}\right) .
$$

Note that it is also possible to construct another linear multiplet

$$
\mathcal{L}^{i j}=\frac{\mathrm{i}}{4}\left(4\left(\boldsymbol{\nabla}^{\hat{\alpha}(i} W\right) \boldsymbol{\nabla}_{\hat{\alpha}}^{j)} L+W \boldsymbol{\nabla}^{\hat{\alpha}(i} \nabla_{\hat{\alpha}}^{j)} L+L \boldsymbol{\nabla}^{\hat{\alpha}(i} \nabla_{\hat{\alpha}}^{j)} W\right),
$$

which couples the two-form multiplet to a vector multiplet $W$. The corresponding component actions can be found in [20,23].

\subsection{Large tensor multiplet}

In [20] it was discovered that there also exists the large tensor multiplet, which consists of $16+16$ degrees of freedom. In superspace the large tensor multiplet may be viewed as a generalization of the gauge two-form multiplet in which the constraints (11.37) are weakened. To show this let $\mathcal{L}$ be a superfield constrained in the same way as eq. (11.37a),

$$
\boldsymbol{\nabla}_{\hat{\alpha}}^{(i} \nabla_{\hat{\beta}}^{j)} \mathcal{L}=\frac{1}{4} \varepsilon_{\hat{\alpha} \hat{\beta}} \nabla^{\hat{\gamma}(i} \nabla_{\hat{\gamma}}^{j)} \mathcal{L}
$$

Requiring only the above constraint, it is possible to show that consistency requires us to have [33]

$$
\begin{aligned}
0 & =\Delta\left\{\boldsymbol{\nabla}^{\hat{\gamma}(i} \boldsymbol{\nabla}_{\hat{\gamma}}^{j)}(\mathcal{W} \mathcal{L})+2\left(\boldsymbol{\nabla}^{\hat{\gamma}(i} \mathcal{W}\right) \boldsymbol{\nabla}_{\hat{\gamma}}^{j)} \mathcal{L}\right\} \\
& =\nabla^{\hat{\gamma}(i} \boldsymbol{\nabla}_{\hat{\gamma}}^{j)}(\mathcal{W} \Delta \mathcal{L})+2\left(\boldsymbol{\nabla}^{\hat{\gamma}(i} \mathcal{W}\right) \boldsymbol{\nabla}_{\hat{\gamma}}^{j)} \Delta \mathcal{L}
\end{aligned}
$$

which is automatically satisfied for the gauge two-form multiplet. Here we will take eq. (11.41) as a second constraint on $\mathcal{L}$. The constraints (11.40) and (11.41) allow us to construct a superform framework describing the large tensor multiplet.

We begin by introducing a two-form ${ }^{30} \mathcal{B}$, transforming as

$$
\delta \mathcal{B}=\Lambda \Delta \mathcal{B}+\mathrm{d} \Xi, \quad \Delta \Xi=0,
$$

\footnotetext{
${ }^{29}$ This superfield Lagrangian first appeared in [33] in Minkowski superspace.

${ }^{30} \mathrm{In}$ this subsection $\mathcal{B}$ will be used for the two-form. It is unrelated to the three-form $\mathcal{B}$ used for the $\mathcal{O}(2)$ multiplet.
} 
and an associated three form $\mathcal{H}$

$$
\mathcal{H}=\nabla \mathcal{B}
$$

Imposing the constraints

$$
\mathcal{H}_{\hat{\alpha}}^{i j k} \hat{\beta} \hat{\gamma}=0, \quad \mathcal{H}_{6 \hat{\alpha} \hat{\beta}}^{i j}=-2 \mathrm{i} \varepsilon^{i j} \varepsilon_{\hat{\alpha} \hat{\beta}} \Delta \mathcal{L}
$$

and solving the Bianchi identities yields the components of $\mathcal{H}$ :

$$
\begin{aligned}
\mathcal{H}_{\hat{a}}^{j k} \hat{\beta} \hat{\gamma} & =-2 \mathrm{i} \varepsilon^{j k}\left(\Gamma_{\hat{a}}\right)_{\hat{\beta} \hat{\gamma}} \mathcal{W} \Delta \mathcal{L} \\
\mathcal{H}_{6 \hat{a}} \hat{a}_{\hat{\beta}}^{j} & =\left(\Gamma_{\hat{a}}\right)_{\hat{\beta}}^{\hat{\gamma}} \nabla_{\hat{\gamma}}^{j} \Delta \mathcal{L} \\
\mathcal{H}_{\hat{a} \hat{b} \hat{\gamma}}^{k} & =2\left(\Sigma_{\hat{a} \hat{b}}\right)_{\hat{\gamma}}^{\hat{\delta}} \nabla_{\hat{\delta}}^{k}(\mathcal{W} \Delta \mathcal{L}) \\
\mathcal{H}_{6 \hat{a} \hat{b}} & =\frac{\mathrm{i}}{4}\left(\Sigma_{\hat{a} \hat{b}}\right)^{\hat{\alpha} \hat{\beta}}\left(\nabla_{\hat{\alpha}}^{k} \nabla_{\hat{\beta} k}-4 \mathrm{i} W_{\hat{\alpha} \hat{\beta}}\right) \Delta \mathcal{L} \\
\mathcal{H}_{\hat{a} \hat{b} \hat{c}} & =-\frac{i}{8} \varepsilon_{\hat{a} \hat{b} \hat{c} \hat{d} \hat{e}}\left(\Sigma^{\hat{d} \hat{e}}\right)^{\hat{\alpha} \hat{\beta}}\left(\left(\nabla_{\hat{\alpha}}^{k} \nabla_{\hat{\beta} k}+4 \mathrm{i} W_{\hat{\alpha} \hat{\beta}}\right)(\mathcal{W} \Delta \mathcal{L})+2\left(\nabla_{\hat{\alpha}}^{k} \mathcal{W}\right) \nabla_{\hat{\beta} k} \Delta \mathcal{L}\right),
\end{aligned}
$$

where $\mathcal{L}$ is constrained by eqs. (11.40) and (11.41) and $\mathcal{H}_{6 \hat{A} \hat{B}}=\Delta \mathcal{B}_{\hat{A} \hat{B}}$. There are still too many component fields and to eliminate them we impose the constraint

$$
\mathcal{B}_{\hat{\alpha} \hat{\beta}}^{i j}=-2 \mathrm{i} \varepsilon^{i j} \varepsilon_{\hat{\alpha} \hat{\beta}} \mathcal{L}
$$

which fixes the remaining components via eq. (11.43) as

$$
\mathcal{B}_{\hat{a} \hat{\beta}}^{j}=\left(\Gamma_{\hat{a}}\right)_{\hat{\beta}}^{\hat{\gamma}} \nabla_{\hat{\gamma}}^{j} \mathcal{L}, \quad \mathcal{B}_{\hat{a} \hat{b}}=\frac{\mathrm{i}}{4}\left(\Sigma_{\hat{a} \hat{b}}\right)^{\hat{\alpha} \hat{\beta}}\left(\nabla_{\hat{\alpha}}^{k} \nabla_{\hat{\beta} k}-4 \mathrm{i} W_{\hat{\alpha} \hat{\beta}}\right) \mathcal{L}
$$

At the highest dimension eq. (11.43) gives

$$
\begin{aligned}
3\left(\nabla_{[\hat{a}}^{\prime} \mathcal{B}_{\hat{b} \hat{c}]}-\mathscr{T}_{[\hat{a} \hat{b} l}^{\hat{\delta}} \mathcal{B}_{c] \hat{\delta}}^{l}\right)=-\frac{\mathrm{i}}{8} \varepsilon_{\hat{a} \hat{b} \hat{c} \hat{d} \hat{e}}\left(\sum^{\hat{d} \hat{e}}\right)^{\hat{\alpha} \hat{\beta}} \Delta & \left(\left(\nabla_{\hat{\alpha}}^{k} \nabla_{\hat{\beta} k}+4 \mathrm{i} W_{\hat{\alpha} \hat{\beta}}\right)(\mathcal{W} \mathcal{L})\right. \\
& \left.+2\left(\nabla_{\hat{\alpha}}^{k} \mathcal{W}\right) \nabla_{\hat{\beta} k} \mathcal{L}\right),
\end{aligned}
$$

where

$$
\nabla_{\hat{a}}^{\prime}=\nabla_{\hat{a}}-\frac{1}{8} \varepsilon_{\hat{a} \hat{b} \hat{c} \hat{d} \hat{e}} W^{\hat{b} \hat{c}} M^{\hat{d} \hat{e}} .
$$

The conditions (11.41) and (11.48) are similar to the ones imposed in [20] from requiring closure of the supersymmetry transformations. In contrast with the gauge two-form multiplet, which was based on the stronger constraints (11.37), the component fields of the large tensor multiplet

$$
\Delta \nabla_{\alpha}^{i} \mathcal{L}\left|, \quad \Delta^{2} \mathcal{L}\right|
$$

are no longer composite.

We should remark that the above constraints can be naturally generalized to include couplings to the Yang-Mills multiplet. Furthermore, since $\mathcal{B}$ possesses the gauge transformation law

$$
\delta \mathcal{B}=\mathrm{d} \Xi, \quad \Delta \Xi=0
$$


one can always shift $\mathcal{L}$ by an abelian vector multiplet

$$
\mathcal{L} \rightarrow \mathcal{L}+c W
$$

where $c$ is an arbitrary real coefficient. One can check that the constraints (11.40) and (11.41) are invariant under such transformations.

We can construct an action for an even number of large tensor multiplets $\mathcal{L}^{I}$. To do so we make use of the superfield Lagrangian

$$
\mathcal{L}^{i j}=\mathcal{L}_{\text {kin }}^{i j}+\mathcal{L}_{\text {mass }}^{i j},
$$

where

$$
\begin{aligned}
\mathcal{L}_{\text {mass }}^{i j} & =\frac{\mathrm{i}}{2} m_{I J}\left(2\left(\boldsymbol{\nabla}^{\hat{\alpha}(i} \mathcal{L}^{I}\right) \nabla_{\hat{\alpha}}^{j)} \mathcal{L}^{J}+\mathcal{L}^{I} \nabla^{\hat{\alpha}(i} \nabla_{\hat{\alpha}}^{j)} \mathcal{L}^{J}\right), & m_{I J}=m_{J I}, \\
\mathcal{L}_{\text {kin }}^{i j} & =\frac{\mathrm{i}}{4} k_{I J}\left(2\left(\boldsymbol{\nabla}^{\hat{\alpha}(i} \mathcal{L}^{I}\right) \overleftrightarrow{\Delta} \nabla_{\hat{\alpha}}^{j)} \mathcal{L}^{J}+\mathcal{L}^{I} \overleftrightarrow{\Delta} \nabla^{\hat{\alpha}(i} \nabla_{\hat{\alpha}}^{j)} \mathcal{L}^{J}\right), & k_{I J}=-k_{J I}
\end{aligned}
$$

The constant matrices $m_{I J}$ and $k_{I J}$ are assumed to be nonsingular. The Lagrangian $\mathcal{L}^{i j}$ may be seen to be a linear multiplet. The component action in supergravity is given in [20].

\section{Off-shell (gauged) supergravity}

We now turn to an off-shell formulation for 5D minimal supergravity obtained by coupling the Weyl multiplet to the following compensators: (i) the vector multiplet; and (ii) the $\mathcal{O}(2)$ multiplet. This is the $5 \mathrm{D}$ analogue of the off-shell formulation for $4 \mathrm{D} \mathcal{N}=2$ supergravity proposed by de Wit, Philippe and Van Proeyen [81]. ${ }^{31}$ We will first describe the construction within superspace and then briefly give the bosonic part of the component action.

\subsection{Superspace formulation}

The superfield Lagrangian for 5D (gauged) supergravity is analogous to the one for 4D $\mathcal{N}=2$ supergravity [83] and reads

$$
\mathcal{L}_{\mathrm{SG}}^{(2)}=\frac{1}{4} V H_{\mathrm{VM}}^{(2)}+G^{(2)} \ln \frac{G^{(2)}}{\mathrm{i}^{(1)} \breve{\Upsilon}^{(1)}}+\kappa V G^{(2)} \equiv \mathcal{L}_{\mathrm{V}}^{(2)}+\mathcal{L}_{\mathrm{L}}^{(2)}+\mathcal{L}_{\mathrm{VL}}^{(2)}
$$

In the first term, $H_{\mathrm{VM}}^{(2)}$ denotes the composite $\mathcal{O}(2)$ multiplet (5.40). The superspace action generated by $\mathcal{L}_{\mathrm{V}}^{(2)}$ then leads to the abelian Chern-Simons action, but normalized with the wrong sign (as usual for a compensator action) and with an additional factor of 3 for later convenience (compare with eq. (9.1)).

Modulo a similar overall sign, the second term in (12.1) denoted by $\mathcal{L}_{\mathrm{L}}^{(2)}$ describes the dynamics of the $\mathcal{O}(2)$ multiplet or, equivalently, linear multiplet without central charge. The superfield $\Upsilon^{(1)}(v)$ is a covariant weight-one arctic multiplet, and $\breve{\Upsilon}^{(1)}(v)$ its smileconjugated antarctic superfield. The action proves to be independent of $\Upsilon^{(1)}$ and $\breve{\Upsilon}^{(1)}$ [83].

\footnotetext{
${ }^{31}$ The $4 \mathrm{D} \mathcal{N}=2$ supergravity formulation of [81] makes use of the $\mathcal{N}=2$ improved tensor multiplet constructed in terms of $\mathcal{N}=1$ superfields in Minkowski superspace [82] and then in terms of component fields in the locally supersymmetric case [81].
} 
The BF term in (12.1) denoted by $\mathcal{L}_{\mathrm{VL}}^{(2)}$ describes a supersymmetric cosmological term. For $\kappa=0$ the Lagrangian (12.1) describes pure Poincaré supergravity, while the case $\kappa \neq 0$ corresponds to gauged or anti-de Sitter supergravity.

Making use of $(7.16)$, the action generated by $\mathcal{L}_{\mathrm{V}}^{(2)}$ may be rewritten as an integral over the full superspace,

$$
S\left[\mathcal{L}_{\mathrm{V}}^{(2)}\right]=\frac{1}{2 \pi} \oint(v, \mathrm{~d} v) \int \mathrm{d}^{5 \mid 8} z E C^{(-4)} \mathcal{L}^{(2)}=\frac{1}{4} \int \mathrm{d}^{5 \mid 8} z E V_{i j} H_{\mathrm{VM}}^{i j},
$$

with $V_{i j}$ being Mezincescu's prepotential. Applying (7.16) once more gives another representation

$$
S\left[\mathcal{L}_{\mathrm{V}}^{(2)}\right]=\frac{1}{4} \int \mathrm{d}^{5 \mid 8} z E \boldsymbol{\Omega}_{\mathrm{VM}} W,
$$

where we have introduced the primary superfield

$$
\boldsymbol{\Omega}_{\mathrm{VM}}=\frac{\mathrm{i}}{4}\left(W \nabla^{i j} V_{i j}-2\left(\nabla^{\hat{\alpha} i} V_{i j}\right) \nabla_{\hat{\alpha}}^{j} W-2 V_{i j} \nabla^{i j} W\right)
$$

which is a prepotential for $H_{\mathrm{VM}}^{(2)}$ in the sense of (7.5b). The representations (12.2) and (12.3) allow us to compute the variation of $S\left[\mathcal{L}_{\mathrm{V}}^{(2)}\right]$ induced by an arbitrary variation of the vector multiplet prepotential, either Mezincescu's or the tropical one,

$$
\begin{aligned}
\delta S\left[\mathcal{L}_{\mathrm{V}}^{(2)}\right] & =\frac{3}{4} \int \mathrm{d}^{5 \mid 8} z E \delta V_{i j} H_{\mathrm{VM}}^{i j} \\
& =\frac{3}{8 \pi} \oint(v, \mathrm{~d} v) \int \mathrm{d}^{5 \mid 8} z E C^{(-4)} \delta V H_{\mathrm{VM}}^{(2)} .
\end{aligned}
$$

Making use of (12.5b), we readily find the equation of motion for the vector multiplet in the supergravity theory (12.1) to be

$$
H_{\mathrm{VM}}^{(2)}+\frac{4 \kappa}{3} G^{(2)}=0 .
$$

We next consider the action generated by $\mathcal{L}_{\mathrm{L}}^{(2)}$. It may be rewritten as an integral over the full superspace

$$
S\left[\mathcal{L}_{\mathrm{L}}^{(2)}\right]=\int \mathrm{d}^{5 \mid 8} z E \Omega \mathbb{W}
$$

where

$$
\mathbb{W}:=-\frac{\mathrm{i}}{16 \pi} \oint(v, \mathrm{~d} v) \nabla^{(-2)} \log \left(\frac{G^{(2)}}{\mathrm{i} \Upsilon^{(1)} \breve{\Upsilon}^{(1)}}\right)
$$

is a composite vector multiplet field strength obeying the Bianchi identity (7.13). The direct evaluation of $\mathbb{W}$ will be given in section 14.2 . The result is

$$
\mathbb{W}=\frac{\mathrm{i}}{16} G \nabla^{\hat{\alpha} i} \nabla_{\hat{\alpha}}^{j}\left(\frac{G_{i j}}{G^{2}}\right) .
$$

It may be seen that varying the prepotential $\Omega$ leads to the following variation of the action:

$$
\delta S\left[\mathcal{L}_{\mathrm{L}}^{(2)}\right]=\int \mathrm{d}^{5 \mid 8} z E \delta \Omega \mathbb{W} .
$$


Finally, we note that the action generated by $\mathcal{L}_{\mathrm{VL}}^{(2)}$ may also be rewritten as an integral over the full superspace

$$
S\left[\mathcal{L}_{\mathrm{VL}}^{(2)}\right]=\kappa \int \mathrm{d}^{5 \mid 8} z E V_{i j} G^{i j}=\kappa \int \mathrm{d}^{5 \mid 8} z E \Omega W
$$

As a result, the complete (gauged) supergravity action becomes

$$
\begin{aligned}
S_{\mathrm{SG}} & =\int \mathrm{d}^{5 \mid 8} z E\left\{\frac{1}{4} V_{i j} H_{\mathrm{VM}}^{i j}+\Omega \mathbb{W}+\kappa V_{i j} G^{i j}\right\} \\
& =\int \mathrm{d}^{5 \mid 8} z E\left\{\frac{1}{4} V_{i j} H_{\mathrm{VM}}^{i j}+\Omega \mathbb{W}+\kappa \Omega W\right\} .
\end{aligned}
$$

Now, from the relations (12.10) and (12.12b) we deduce the supergravity equation of motion for the $\mathcal{O}(2)$ compensator:

$$
\mathbb{W}+\kappa W=0
$$

The equation of motion for the Weyl multiplet is

$$
G-W^{3}=0 .
$$

It may be shown that, modulo gauge freedom, the Weyl multiplet is described by a single unconstrained real prepotential $\mathfrak{U}^{32}$ The equation (12.14) is obtained by varying the supergravity action with respect to $\mathfrak{U}$. The meaning of (12.14) is that the supercurrent of pure supergravity is equal to zero.

In general, given a dynamical system involving (matter) superfields $\varphi^{i}$ coupled to the Weyl multiplet, the supercurrent of this theory is a dimension-3 primary real scalar superfield defined by

$$
\mathcal{T}=\frac{\Delta}{\Delta \mathfrak{U}} S[\varphi]
$$

where $\Delta / \Delta \mathfrak{U}$ denotes a covariantized variational derivative with respect to $\mathfrak{U}$. The variation $\Delta \mathfrak{U}$ is a primary superfield with dimension -2 . The supercurrent turns out to satisfy the conservation equation

$$
\nabla^{i j} \mathcal{T}=0
$$

provided the dynamical superfields obey their equations of motion, $\delta S[\varphi] / \delta \varphi^{i}=0$. This follows from the fact that $\Delta \mathfrak{U}$ is defined modulo gauge transformations

$$
\Delta \mathfrak{U} \rightarrow \Delta \mathfrak{U}+\nabla^{i j} \Omega_{i j}
$$

where gauge parameter $\Omega_{i j}$ is a primary real isovector superfield with dimension -3 .

It is an instructive exercise to prove that the left-hand side of (12.14) obeys the constraint

$$
\nabla^{i j}\left(G-W^{3}\right)=0
$$

provided the equations (12.6) and (12.13) hold.

The supergravity equations of motion (12.6), (12.13) and (12.14) appeared in [85]. They are analogous to the superfield equations for $4 \mathrm{D} \mathcal{N}=2$ (gauged) supergravity $[69,86]$.

\footnotetext{
${ }^{32}$ This can be done in complete analogy with the case of $4 \mathrm{D} \mathcal{N}=2$ supergravity [84].
} 


\subsection{Component formulation}

To complement the superspace discussion, we now present briefly the bosonic part of the component action for gauged supergravity. The three superspace actions given in (12.1) can be analyzed in components easily using results given elsewhere in this paper. The first term $\mathcal{L}_{\mathrm{V}}^{(2)}$ leads to the wrong sign abelian Chern-Simons Lagrangian

$$
\begin{aligned}
\mathcal{L}_{\mathrm{V}}= & \frac{1}{8} \varepsilon^{\hat{a} \hat{b} \hat{c} \hat{d} \hat{e}} v_{\hat{a}} f_{\hat{b} \hat{c}} f_{\hat{d} \hat{e}}+\frac{3}{4} W f_{\hat{a} \hat{b}} f^{\hat{a} \hat{b}}-\frac{3}{4} W X^{i j} X_{i j}+\frac{9}{4} w^{\hat{a} \hat{b}} f_{\hat{a} \hat{b}} W^{2} \\
& +\frac{3}{2} W\left(\hat{\mathcal{D}}^{\hat{a}} W\right) \hat{\mathcal{D}}_{\hat{a}} W-\frac{1}{8} \hat{\mathcal{R}} W^{3}+4 D W^{3}+\frac{39}{32} w^{\hat{a} \hat{b}} w_{\hat{a} \hat{b}} W^{3}
\end{aligned}
$$

where $\hat{\mathcal{D}}_{\hat{a}}$ is defined by eq. (4.32). The second term $\mathcal{L}_{\mathrm{L}}^{(2)}$ leads to the $\mathcal{O}(2)$ multiplet Lagrangian

$$
\begin{aligned}
\mathcal{L}_{\mathrm{L}}= & \frac{1}{4} G^{-1}\left(\hat{\mathcal{D}}_{\hat{a}} G^{i j}\right) \hat{\mathcal{D}}^{\hat{a}} G_{i j}-\frac{1}{2} G^{-1} \phi^{\hat{a}} \phi_{\hat{a}} \\
& +\frac{1}{12} \varepsilon^{\hat{a} \hat{b} \hat{c} \hat{d} \hat{e}} b_{\hat{c} \hat{d} \hat{e}}\left(\frac{1}{2} G^{-3}\left(\hat{\mathcal{D}}_{\hat{a}} G_{i k}\right)\left(\hat{\mathcal{D}}_{\hat{b}} G_{j}{ }^{k}\right) G^{i j}+G^{-1} \hat{R}(J)_{\hat{a} \hat{b}}{ }^{i j} G_{i j}\right) \\
& -\frac{1}{8 G} F^{2}-\frac{3}{8} \hat{\mathcal{R}} G-4 D G-\frac{3}{32} w^{\hat{a} \hat{b}} w_{\hat{a} \hat{b}} G .
\end{aligned}
$$

This Lagrangian is analogous to the 4D improved tensor multiplet Lagrangian [81] and shares similar features. In particular, the second line of (12.20) involves a BF coupling between the three-form $b_{\hat{a} \hat{b} \hat{c}}$ and a composite two-form constructed from the tensor multiplet scalars and the $\mathrm{SU}(2)$ gauge fields. As discussed in [81], this two-form is closed but not exact: it has no $\mathrm{SU}(2)$-invariant one-form potential. The third superspace Lagrangian $\mathcal{L}_{\mathrm{VL}}^{(2)}$ leads to the simple expression

$$
\mathcal{L}_{\mathrm{VL}}=-\kappa W F-\kappa X^{i j} G_{i j}-2 \kappa v_{\hat{a}} \phi^{\hat{a}} .
$$

We now combine all three Lagrangians and eliminate the auxiliary fields using their equations of motion. The equation of motion for $D$ is

$$
W^{3}-G=0
$$

and corresponds to the lowest component of the superfield equation of motion (12.14). Similarly, the equations of motion for the vector multiplet auxiliary $X^{i j}$ and the $\mathcal{O}(2)$ multiplet auxiliary $F$ lead, respectively, to

$$
\begin{aligned}
\frac{3}{2} W X_{i j}+\kappa G_{i j} & =0, \\
\frac{1}{4 G} F+\kappa W & =0,
\end{aligned}
$$

which correspond to the bosonic parts of the lowest components of (12.6) and (12.13), respectively. Finally, we must impose the equation of motion for $w_{\hat{a} \hat{b}}$, which leads to

$$
w_{\hat{a} \hat{b}} W+f_{\hat{a} \hat{b}}=0 .
$$


This is actually the bosonic part of a higher component of the Weyl superfield equation of motion; it can be extracted by applying $\nabla_{(\hat{\alpha}}^{k} \nabla_{\hat{\beta}) k}$ to (12.14) and taking the lowest component.

After imposing each of these equations, we finally choose the Weyl gauge $W=1$. This leads to the component Lagrangian

$$
\begin{aligned}
\mathcal{L}_{\mathrm{SG}}= & -\frac{1}{2} \hat{\mathcal{R}}+\frac{1}{8} \varepsilon^{\hat{a} b \hat{c} \hat{d} \hat{e}} v_{\hat{a}} f_{\hat{b} \hat{c}} f_{\hat{d} \hat{e}}-\frac{3}{8} f_{\hat{a} \hat{b}} f^{\hat{a} \hat{b}}+\frac{8}{3} \kappa^{2} \\
& +\frac{1}{4}\left(\hat{\mathcal{D}}_{\hat{a}} G^{i j}\right) \hat{\mathcal{D}}^{\hat{a}} G_{i j}-\frac{1}{2} \phi^{\hat{a}} \phi_{\hat{a}}-2 \kappa v_{\hat{a}} \phi^{\hat{a}} \\
& +\frac{1}{12} \varepsilon^{\hat{a} \hat{b} \hat{c} \hat{d} \hat{e}} b_{\hat{c} \hat{d} \hat{e}}\left(\frac{1}{2}\left(\hat{\mathcal{D}}_{\hat{a}} G_{i k}\right)\left(\hat{\mathcal{D}}_{\hat{b}} G_{j}{ }^{k}\right) G^{i j}+\hat{R}(J)_{\hat{a} \hat{b}}^{i j} G_{i j}\right) .
\end{aligned}
$$

The terms in the second and third lines turn out to lead to auxiliary fields. The easiest way to see this is to adopt the $\mathrm{SU}(2)$ gauge

$$
G_{\underline{12}}=\mathrm{i}, \quad G_{\underline{11}}=G_{\underline{22}}=0,
$$

which breaks the R-symmetry group to U(1). Using

$$
\hat{\mathcal{D}}_{\hat{a}} G^{11}=2 \mathrm{i} \mathcal{V}_{\hat{a}}^{\underline{11}}, \quad \hat{\mathcal{D}}_{\hat{a}} G^{22}=-2 \mathrm{i} \mathcal{V}_{\hat{a}}^{\underline{22}}, \quad \hat{\mathcal{D}}_{\hat{a}} G^{12}=0,
$$

the supergravity Lagrangian can be rewritten as

$$
\begin{aligned}
& \mathcal{L}_{\mathrm{SG}}=-\frac{1}{2} \hat{\mathcal{R}}+\frac{1}{8} \varepsilon^{\hat{a} \hat{b} \hat{c} \hat{d} \hat{e}} v_{\hat{a}} f_{\hat{b} \hat{c}} f_{\hat{d} \hat{e}}-\frac{3}{8} f_{\hat{a} \hat{b}} f^{\hat{a} \hat{b}}+\frac{8}{3} \kappa^{2} \\
& +2 \mathcal{V}_{\hat{a}}^{\underline{11}} \mathcal{V}^{\hat{a} \underline{22}}-\frac{1}{2} \phi^{\hat{a}} \phi_{\hat{a}}-2\left(\kappa v_{\hat{a}}+\mathrm{i} \mathcal{V}_{\hat{a}}^{\underline{12}}\right) \phi^{\hat{a}} \text {. }
\end{aligned}
$$

Now one introduces a Lagrange multiplier term $\phi^{\hat{a}} \hat{\mathcal{D}}_{\hat{a}} \lambda$ to enforce the constraint on $\phi^{\hat{a}}$; the field $\lambda$ is eaten by $\mathcal{V}_{\hat{a}} \underline{12}$, which fixes the remaining R-symmetry up to a compensating $\kappa$ dependent transformation to counter the graviphoton's gauge transformation. Integrating out $\phi_{\hat{a}}$ then gives

$$
\mathcal{L}_{\mathrm{SG}}=-\frac{1}{2} \hat{\mathcal{R}}+\frac{1}{8} \varepsilon^{\hat{a} \hat{a} \hat{c} \hat{d} \hat{e}} v_{\hat{a}} f_{\hat{b} \hat{c}} f_{\hat{d} \hat{e}}-\frac{3}{8} f_{\hat{a} \hat{b}} f^{\hat{a} \hat{b}}+\frac{8}{3} \kappa^{2}+\left(\mathcal{V}_{\hat{a}}^{i j}+\kappa G^{i j} v_{\hat{a}}\right)^{2}
$$

where we have written the auxiliary one-forms in a way which holds for any choice of constant $G^{i j}$. The equation of motion for this auxiliary then fixes $\mathcal{V}_{\hat{a}}{ }^{i j}=-\kappa G^{i j} v_{\hat{a}}$, which is ultimately responsible for the $\kappa$-dependent minimal coupling between the gravitino and the graviphoton. Note that the cosmological constant is given in these conventions by

$$
\Lambda=-\frac{8}{3} \kappa^{2}<0
$$

\section{Dilaton Weyl multiplets and superforms}

It is possible to construct variant formulations for conformal supergravity by coupling the standard Weyl multiplet, which is described in sections 2 and 4, to an on-shell abelian 
vector multiplet with nowhere vanishing field strength, $W \neq 0$. The field strength $W$ of such a vector multiplet satisfies the Bianchi identity (2.49) as well as the equation of motion

$$
\mathbb{H}^{i j}=0
$$

derived from a gauge invariant action $S[W]$, see section 6.6.

In this section, we consider a special case of the equation of motion (13.1) that originates in 5D minimal supergravity with cosmological term realized as conformal supergravity coupled to two compensators: (i) the vector multiplet; and (ii) the $\mathcal{O}(2)$ multiplet. In this case $\mathbb{H}^{i j} \equiv H_{\mathrm{VM}}^{i j}$, where $H_{\mathrm{VM}}^{i j}$ denotes the composite Yang-Mills $\mathcal{O}(2)$ multiplet (2.54). In the superspace setting, the supergravity equations of motion [85] are given by eqs. (12.6), (12.13) and (12.14). In what follows, we will only use eq. (12.6).

\subsection{The dilaton Weyl multiplet}

The dilaton Weyl multiplet ${ }^{33}[19,21]$ is equivalently described as the standard Weyl multiplet coupled to a vector multiplet compensator obeying the equation of motion

$$
H_{\mathrm{VM}}^{i j}=0 .
$$

The formulation of this multiplet in $\mathrm{SU}(2)$ superspace was given in [3]. Eq. (13.2) in $\mathrm{SU}(2)$ superspace is equivalent to

$$
S^{i j}=\frac{\mathrm{i}}{2 W^{2}}\left\{\left(\mathcal{D}^{\hat{\alpha}(i} W\right) \mathcal{D}_{\hat{\alpha}}^{j)} W+\frac{1}{2} W \mathcal{D}^{i j} W\right\} .
$$

Equation (13.2) tells us that the matter fields of the super Weyl tensor $W_{\hat{a} \hat{b}}$ satisfy certain constraints that allow one to solve $W_{\hat{a} \hat{b}}$ in terms of a gauge two-form. To see this we make use of the equivalence between vector and two-form multiplets on the mass shell. We recall that the two-form multiplet was described in section 11.3 and here we will use its superform realization.

Ignoring the Chern-Simons couplings to Yang-Mills multiplets, a two-form multiplet possesses a gauge two-form $\mathscr{B}$ with corresponding field strength

$$
\mathscr{H}=\mathrm{d} \mathscr{B}-\mathcal{V} \wedge \Delta \mathscr{B} .
$$

Imposing the on-shell condition for a two-form multiplet, $\Delta L=0$, allows one to identify $L$ with a vector multiplet. In fact, we identify both the vector multiplet gauging the central charge and the two-form multiplet with the same vector multiplet. To do this we make the replacements

$$
L \rightarrow-W, \quad \mathcal{W} \rightarrow W
$$

which requires

$$
\Delta \mathscr{B}=F, \quad \mathcal{F}=F, \quad \mathcal{V}=V .
$$

\footnotetext{
${ }^{33}$ The dilaton Weyl multiplet corresponds to the Nishino-Rajpoot version [87] of 5D $\mathcal{N}=1$ Poincaré supergravity.
} 
Note that the gauge transformations become

$$
\delta V=\mathrm{d} \Lambda, \quad \delta \mathscr{B}=\Lambda F+\mathrm{d} \Xi .
$$

The field strength $\mathscr{H}=\mathrm{d} \mathscr{B}-V \wedge F$ satisfies the Bianchi identity

$$
\mathrm{d} \mathscr{H}=-F \wedge F,
$$

or, equivalently,

$$
\nabla_{[\hat{A}} \mathscr{H}_{\hat{B} \hat{C} \hat{D}\}}+\frac{3}{2} \mathscr{T}_{[\hat{A} \hat{B}} \hat{E} \mathscr{H}_{|\hat{E}| \hat{C} \hat{D}\}}+\frac{3}{2} F_{[\hat{A} \hat{B}} F_{\hat{C} \hat{D}\}}=0 .
$$

The solution to the above Bianchi identity may be read off of eq. (11.36):

$$
\begin{aligned}
\mathscr{H}_{\hat{\alpha}}^{i j} \hat{\beta} \hat{\gamma} & =0 \\
\mathscr{H}_{\hat{a}}^{j k} \hat{\beta} & =2 \mathrm{i} \varepsilon^{j k}\left(\Gamma_{\hat{a}}\right)_{\hat{\beta} \hat{\gamma}} W^{2}, \\
\mathscr{H}_{\hat{a} \hat{b} \hat{\gamma}}^{k} & =-2\left(\Sigma_{\hat{a} \hat{b}}\right)_{\hat{\gamma}}^{\hat{\gamma}} \nabla_{\hat{\delta}}^{k} W^{2}, \\
\mathscr{H}_{\hat{a} \hat{b} \hat{c}} & =\frac{\mathrm{i}}{8} \varepsilon_{\hat{a} \hat{b} \hat{c} \hat{d} \hat{e}}\left(\Sigma^{\hat{d} \hat{e}}\right)^{\hat{\alpha} \hat{\beta}}\left(\nabla_{\hat{\alpha}}^{k} \nabla_{\hat{\beta} k} W^{2}+4 \mathrm{i} W_{\hat{\alpha} \hat{\beta}} W^{2}+2\left(\nabla_{\hat{\alpha}}^{k} W\right) \nabla_{\hat{\beta} k} W\right) .
\end{aligned}
$$

The Bianchi identities are satisfied since we have the on-shell condition (13.2), which is equivalent to

$$
\nabla^{\hat{\gamma}(i} \nabla_{\hat{\gamma}}^{j)} W^{2}=-2\left(\nabla^{\hat{\gamma}(i} W\right) \nabla_{\hat{\gamma}}^{j)} W
$$

From the component $\mathscr{H}_{\hat{a} \hat{b} \hat{c}}$ one finds the expression for the super Weyl tensor

$$
W_{\hat{a} \hat{b}}=\frac{1}{3 W^{2}}\left(\frac{1}{6} \varepsilon_{\hat{a} \hat{b} \hat{c} \hat{d} \hat{e}} \mathscr{H}^{\hat{c} \hat{d} \hat{e}}-2 W F_{\hat{a} \hat{b}}-\mathrm{i}\left(\Sigma_{\hat{a} \hat{b}}\right)^{\hat{\alpha} \hat{\beta}} \lambda_{\hat{\alpha}}^{k} \lambda_{\hat{\beta} k}\right) .
$$

Due to the above relation we see that we may instead choose the gauge two-form $\mathscr{B}_{\hat{a} \hat{b}}$ as a fundamental component field. This means that the matter fields in the standard Weyl multiplet become composite. They may be derived directly from the above superspace expression for $W_{\hat{a} \hat{b}}$.

Using the above relations we can replace the matter fields in the standard Weyl multiplet:

$$
\left(W_{\hat{a} \hat{b}}, X_{\hat{\alpha}}^{i}, Y\right) \rightarrow\left(W, \lambda_{\hat{\alpha}}^{i}, V_{\hat{m}}, \mathscr{B}_{\hat{m} \hat{n}}\right) .
$$

This leads to the dilaton Weyl multiplet, which only differs from the standard Weyl multiplet in the matter field content. One can check that both Weyl multiplets contain $32+32$ degrees of freedom.

The construction of actions involving the dilaton Weyl multiplet may be readily obtained from those involving the standard Weyl multiplet upon making the replacements in this subsection. One can further construct actions by replacing any vector multiplet $\hat{W}$ with the components of the dilaton Weyl multiplet as follows:

$$
\left(\hat{W}, \hat{\lambda}_{\hat{\alpha}}^{i}, \hat{V}_{\hat{m}}, \hat{X}^{i j}\right) \rightarrow\left(W, \lambda_{\hat{\alpha}}^{i}, V_{\hat{m}}, \frac{\mathrm{i}}{2 W} \lambda^{i} \lambda^{j}\right)
$$




\subsection{The deformed dilaton Weyl multiplet}

The deformed Weyl multiplet [88] is equivalently described as the standard Weyl multiplet coupled to a vector multiplet compensator obeying the equation of motion

$$
H_{\mathrm{VM}}^{i j}=-\frac{4 \kappa}{3} G^{i j}
$$

which implies

$$
X^{i j}=\frac{\mathrm{i}}{2 W} \lambda^{i} \lambda^{j}-\frac{2 \kappa}{3 W} G^{i j} .
$$

Here the $\mathcal{O}(2)$ compensator $G^{i j}$ is considered as a background field.

Just like in the previous case we can give the constrained system a geometric description. We now modify the superform equation to

$$
\mathrm{d} \mathscr{H}=-F \wedge F-\frac{4 \kappa}{3} \Phi
$$

where

$$
\mathscr{H}=\mathrm{d} \mathscr{B}-V \wedge F-\frac{4 \kappa}{3} \mathcal{B} .
$$

Here $\mathcal{B}$ is the gauge three-form for the $\mathcal{O}(2)$ multiplet. From the above we see that we must modify the gauge transformation of $\mathscr{B}$ to be

$$
\delta \mathscr{B}=\Lambda F+\mathrm{d} \Xi+\frac{4 \kappa}{3} \rho
$$

since

$$
\delta \mathcal{B}=\mathrm{d} \rho
$$

The solution is

$$
\begin{aligned}
\mathscr{H}_{\hat{\alpha} \hat{\beta} \hat{\gamma}}^{i j k} & =0, \\
\mathscr{H}_{\hat{a}}^{j k} \hat{\beta} \hat{\gamma} & =2 \mathrm{i} \varepsilon^{j k}\left(\Gamma_{\hat{a}}\right)_{\hat{\beta} \hat{\gamma}} W^{2}, \\
\mathscr{H}_{\hat{a} \hat{b} \hat{\gamma}}^{k} & =-2\left(\Sigma_{\hat{a} \hat{b}}\right)_{\hat{\gamma}}^{\hat{\gamma}} \nabla_{\hat{\delta}}^{k} W^{2}, \\
\mathscr{H}_{\hat{a} \hat{b} \hat{c}} & =\frac{\mathrm{i}}{8} \varepsilon_{\hat{a} \hat{b} \hat{c} \hat{d} \hat{e}}\left(\Sigma^{\hat{d} \hat{e}}\right)^{\hat{\alpha} \hat{\beta}}\left(\nabla_{\hat{\alpha}}^{k} \nabla_{\hat{\beta} k} W^{2}+4 \mathrm{i} W_{\hat{\alpha} \hat{\beta}} W^{2}+2\left(\nabla_{\hat{\alpha}}^{k} W\right) \nabla_{\hat{\beta} k} W\right) .
\end{aligned}
$$

From the component $\mathscr{H}_{\hat{a} \hat{b} \hat{c}}$ one finds the expression

$$
W_{\hat{a} \hat{b}}=\frac{1}{3 W^{2}}\left(\frac{1}{6} \varepsilon_{\hat{a} \hat{b} \hat{c} \hat{d} \hat{e}} \mathscr{H}^{\hat{c} \hat{d} \hat{e}}-2 W F_{\hat{a} \hat{b}}-\mathrm{i}\left(\Sigma_{\hat{a} \hat{b}}\right)^{\hat{\alpha} \hat{\beta}} \lambda_{\hat{\alpha}}^{k} \lambda_{\hat{\beta} k}\right) .
$$

Again the matter components of the Weyl multiplet may be replaced using the above expression. The above expression for $W_{\hat{a} \hat{b}}$ looks formally the same as eq. (13.12). However, it should be kept in mind that $W$ now satisfies the different on-shell constraint

$$
\nabla^{\hat{\gamma}(i} \nabla_{\hat{\gamma}}^{j)} W^{2}=-2\left(\nabla^{\hat{\gamma}(i} W\right) \nabla_{\hat{\gamma}}^{j)} W+\frac{16 \mathrm{i}}{3} \kappa G^{i j}
$$




\subsection{The deformed dilaton Weyl multiplet with Chern-Simons couplings}

It was mentioned in [26] that one can generalize the construction of the dilaton Weyl multiplet to include a system of abelian vector multiplets. Using a similar idea we generalize the deformed dilaton Weyl multiplet of the previous subsection in the presence of YangMills couplings.

We now modify the superform equation to

$$
\mathrm{d} \mathscr{H}=-F \wedge F-\operatorname{tr}(\boldsymbol{F} \wedge \boldsymbol{F})-\frac{4 \kappa}{3} \Phi,
$$

where

$$
\mathscr{H}=\mathrm{d} \mathscr{B}-V \wedge F-\operatorname{tr}\left(\boldsymbol{V} \wedge \boldsymbol{F}-\frac{\mathrm{i}}{3} \boldsymbol{V} \wedge \boldsymbol{V} \wedge \boldsymbol{V}\right)-\frac{4 \kappa}{3} \mathcal{B} .
$$

From the above we see that we must modify the gauge transformation of $\mathscr{B}$ to be

$$
\delta \mathscr{B}=\Lambda F+\operatorname{tr}(\boldsymbol{\tau} \mathrm{d} \boldsymbol{V})+\mathrm{d} \Xi+\frac{4 \kappa}{3} \rho
$$

since

$$
\delta \mathcal{B}=\mathrm{d} \rho, \quad \delta \boldsymbol{V}=\mathrm{d} \boldsymbol{\tau}-\mathrm{i}[\boldsymbol{V}, \boldsymbol{\tau}] .
$$

The superform equation (13.24) is solved by

$$
\begin{aligned}
\mathscr{H}_{\hat{\alpha} \hat{\beta} \hat{\gamma}}^{i j k} & =0, \\
\mathscr{H}_{\hat{a}}^{j k} \hat{\beta}= & 2 \mathrm{i} \varepsilon^{j k}\left(\Gamma_{\hat{a}}\right)_{\hat{\beta} \hat{\gamma}}\left(W^{2}+\operatorname{tr}\left(\boldsymbol{W}^{2}\right)\right), \\
\mathscr{H}_{\hat{a} \hat{b} \hat{\gamma}}^{k} & =-2\left(\sum_{\hat{a} \hat{b}}\right)_{\hat{\gamma}}^{\hat{\delta}} \nabla_{\hat{\delta}}^{k}\left(W^{2}+\operatorname{tr}\left(\boldsymbol{W}^{2}\right)\right) \\
\mathscr{H}_{\hat{a} \hat{b} \hat{c}} & =\frac{\mathrm{i}}{8} \varepsilon_{\hat{a} \hat{b} \hat{c} \hat{d} \hat{e}}\left(\Sigma^{\hat{d e} \hat{e}}\right)^{\hat{\alpha} \hat{\beta}}\left(\nabla_{\hat{\alpha}}^{k} \nabla_{\hat{\beta} k}\left(W^{2}+\operatorname{tr}\left(\boldsymbol{W}^{2}\right)\right)+4 \mathrm{i} W_{\hat{\alpha} \hat{\beta}} W^{2}\right. \\
& \left.\quad+2 \operatorname{tr}\left(\left(\nabla_{\hat{\alpha}}^{k} \boldsymbol{W}\right) \boldsymbol{\nabla}_{\hat{\beta} k} \boldsymbol{W}\right)+2\left(\nabla_{\hat{\alpha}}^{k} W\right) \nabla_{\hat{\beta} k} W\right)
\end{aligned}
$$

where $W$ satisfies the Bianchi identity

$$
\boldsymbol{\nabla}^{\hat{\gamma}(i} \nabla_{\hat{\gamma}}^{j)}\left(W^{2}+\operatorname{tr}\left(\boldsymbol{W}^{2}\right)\right)=-2\left(\nabla^{\hat{\gamma}(i} W\right) \nabla_{\hat{\gamma}}^{j)} W-2 \operatorname{tr}\left(\left(\boldsymbol{\nabla}^{\hat{\gamma}(i} \boldsymbol{W}\right) \boldsymbol{\nabla}_{\hat{\gamma}}^{j)} \boldsymbol{W}\right)+\frac{16 \mathrm{i}}{3} \kappa G^{i j},
$$

which implies

$$
X^{i j}=\frac{\mathrm{i}}{2 W}\left(\lambda^{i} \lambda^{j}+\operatorname{tr}\left(\boldsymbol{\lambda}^{i} \boldsymbol{\lambda}^{j}\right)\right)-\frac{1}{W} \operatorname{tr}\left(\boldsymbol{W} \boldsymbol{X}^{i j}\right)-\frac{2 \kappa}{3 W} G^{i j} .
$$

From the component $\mathscr{H}_{\hat{a} \hat{b} \hat{c}}$ one finds the expression

$$
\begin{aligned}
& 3\left(W^{2}+\operatorname{tr}\left(\boldsymbol{W}^{2}\right)\right) W_{\hat{a} \hat{b}}=\left(\frac{1}{6} \varepsilon_{\hat{a} \hat{b} \hat{c}} \hat{d} \hat{e}\right. \mathscr{H} \\
&-\mathrm{i}\left(\Sigma_{\hat{a} \hat{b}}\right)^{\hat{c} \hat{e} \hat{e}}-2 W F_{\hat{\alpha} \hat{b}}-2 \operatorname{tr}\left(\boldsymbol{W} \boldsymbol{F}_{\hat{\alpha} \hat{b}}\right) \\
&\left.\lambda_{\hat{\beta} k}-\mathrm{i}\left(\Sigma_{\hat{a} \hat{b}}\right)^{\hat{\alpha} \hat{\beta}} \operatorname{tr}\left(\boldsymbol{\lambda}_{\hat{\alpha}}^{k} \boldsymbol{\lambda}_{\hat{\beta} k}\right)\right) .
\end{aligned}
$$

If $\left(W^{2}+\operatorname{tr}\left(\boldsymbol{W}^{2}\right)\right)$ does not vanish then we can again replace the matter fields of the Weyl multiplet with those of $W$. 
The supersymmetry transformations of the gauge fields may be obtained from eq. (4.42) upon using (13.31). We list the supersymmetry transformations of the matter fields below:

$$
\begin{aligned}
\delta W= & \mathrm{i} \xi_{k} \lambda^{k} \\
\delta \Lambda_{\hat{\alpha}}^{i}= & 2 \xi^{\hat{\beta} i}\left(F_{\hat{\beta} \hat{\alpha}}+W_{\hat{\beta} \hat{\alpha}} W\right)+\frac{\mathrm{i}}{2 W} \xi_{\hat{\alpha} j}\left(\lambda^{(i} \lambda^{j)}+\operatorname{tr}\left(\boldsymbol{\lambda}^{(i} \boldsymbol{\lambda}^{j)}\right)\right)-\frac{1}{W} \xi_{\hat{\alpha} j} \operatorname{tr}\left(\boldsymbol{W} \boldsymbol{X}^{i j}\right) \\
& -\frac{2 \kappa}{3 W} \xi_{\hat{\alpha} j} G^{i j}-\xi^{\hat{\beta} i} \nabla_{\hat{\beta} \hat{\alpha}} W+2 \mathrm{i} \eta_{\hat{\alpha}}^{i} W \\
\delta V_{\hat{m}}= & \mathrm{i} \xi_{k} \Gamma_{\hat{m}} \lambda^{k}+\mathrm{i} \xi_{j} \psi_{\hat{m}}^{j} W \\
\delta \mathscr{B}_{\hat{m} \hat{n}}= & 2 \mathrm{i} \xi_{k} \Gamma_{[\hat{m}} \psi_{\hat{n}]}{ }^{k}\left(W^{2}+\operatorname{tr}\left(\boldsymbol{W}^{2}\right)\right)-4 \mathrm{i} \xi_{k} \Sigma_{\hat{m} \hat{n}} \lambda^{k} W \\
& -4 \mathrm{i} \xi_{k} \Sigma_{\hat{m} \hat{n}} \operatorname{tr}\left(\boldsymbol{\lambda}^{k} \boldsymbol{W}\right)-2 V_{[\hat{m}} \delta V_{\hat{n}]}-2 \operatorname{tr}\left(\boldsymbol{V}_{[\hat{m}} \delta \boldsymbol{V}_{\hat{n}]}\right) .
\end{aligned}
$$

The superconformal field strengths are given by

$$
\begin{aligned}
F_{\hat{a} \hat{b}}= & 2 e_{\hat{a}}^{\hat{m}} e_{\hat{b}}^{\hat{n}} \partial_{[\hat{m}} V_{\hat{n}]}-\mathrm{i} \psi_{[\hat{a} k} \Gamma_{\hat{b}]} \lambda^{k}+\frac{\mathrm{i}}{2} \psi_{[\hat{a} k}^{\hat{\gamma}} \psi_{\hat{b}] \hat{\gamma}}^{k} W \\
\boldsymbol{F}_{\hat{a} \hat{b}}= & 2 e_{\hat{a}}^{\hat{m}} e_{\hat{b}}^{\hat{n}}\left(\partial_{[\hat{m}} \boldsymbol{V}_{\hat{n}]}-\mathrm{i} \boldsymbol{V}_{[\hat{m}} \boldsymbol{V}_{\hat{n}]}\right)-\mathrm{i} \psi_{[\hat{a} k} \Gamma_{\hat{b}]} \boldsymbol{\lambda}^{k}+\frac{\mathrm{i}}{2} \psi_{[\hat{a} k}^{\hat{\gamma}} \psi_{\hat{b}] \hat{\gamma}}^{k} \boldsymbol{W}, \\
\mathscr{H}_{\hat{a} \hat{b} \hat{c}}= & e_{\hat{a}}^{\hat{m}} e_{\hat{b}}^{\hat{n}} e_{\hat{c}}^{\hat{p}}\left(3 \partial_{[\hat{m}} \mathscr{B}_{\hat{n} \hat{p}]}-3 V_{[\hat{m}} F_{\hat{n} \hat{p}]}-\operatorname{tr}\left(3 \boldsymbol{V}_{[\hat{m}} \boldsymbol{F}_{\hat{n} \hat{p}]}+2 \mathrm{i} \boldsymbol{V}_{[\hat{m}} \boldsymbol{V}_{\hat{n}} \boldsymbol{V}_{\hat{p}]}\right)-\frac{4 \kappa}{3} \mathcal{B}_{\hat{m} \hat{n} \hat{p}}\right) \\
& +\frac{3 \mathrm{i}}{2} \psi_{[\hat{a}}^{k} \Gamma_{\hat{b}}^{k} \psi_{\hat{c}] k}\left(W^{2}+\operatorname{tr}\left(\boldsymbol{W}^{2}\right)\right) \\
& -6 \mathrm{i} W \psi_{[\hat{a}}^{k} \Sigma_{\hat{b} \hat{c}]} \lambda_{k}-6 \mathrm{i} \operatorname{tr}\left(\boldsymbol{W} \psi_{[\hat{a}}^{k} \sum_{\hat{b} \hat{c}]} \boldsymbol{\lambda}_{k}\right) .
\end{aligned}
$$

The supersymmetry transformations for the previous two cases (the dilaton Weyl and deformed dilaton Weyl multiplets) may be straightforwardly obtained from the above general results.

\section{Higher derivative couplings}

The superspace formalism developed in this paper offers more general tools to construct composite primary multiplets (that may be used, e.g., to generate higher derivative invariants) than those which have so far been employed within the component approaches [14-23]. This will be demonstrated below.

\subsection{Composite primary multiplets and invariants}

In section 6 we derived two gauge prepotentials for the abelian vector multiplet: (i) the tropical prepotential $V(v)$; and (ii) Mezincescu's prepotential $V_{i j}$. These constructions lead to two different procedures to generate composite vector multiplet field strengths.

Associated with a composite weight- 0 tropical multiplet $\mathbb{V}(v)$ is the following primary real scalar

$$
\mathbb{W}_{\text {tropical }} \equiv W[\mathbb{V}]=-\frac{\mathrm{i}}{16 \pi} \oint(v, \mathrm{~d} v) \nabla^{(-2)} \mathbb{V}(v) .
$$

It obeys the Bianchi identity (7.13). Thus we may think of $\mathbb{W}_{\text {tropical }}$ as the field strength of a composite vector multiplet. An example is provided by

$$
\mathbb{V}=\frac{H^{(2 n)}}{\left[G^{(2)}\right]^{n}}, \quad n=1,2, \ldots,
$$


for an arbitrary real $\mathcal{O}(2 n)$ multiplet $H^{(2 n)}(v)$ and an $\mathcal{O}(2)$ multiplet $G^{(2)}(v)$ such that the scalar $G$ defined by (7.25) is nowhere vanishing. The existence of the latter is assumed in this section.

Associated with a composite real isovector superfield $\mathbb{V}_{i j}$ with dimension -2 is the following primary real scalar

$$
\mathbb{W}_{\text {Mezincescu }} \equiv W\left[\mathbb{V}_{i j}\right]=-\frac{3 \mathrm{i}}{40} \nabla_{i j} \Delta^{i j k l} \mathbb{V}_{k l}
$$

It obeys the Bianchi identity (7.13). As an example, we consider

$$
\mathbb{V}_{i j}=\frac{G_{i j}}{G^{5 / 3}}
$$

In section 7 we derived the unconstrained prepotential $\Omega$ for the $\mathcal{O}(2)$ multiplet. This construction leads to a procedure to generate composite $\mathcal{O}(2)$ multiplets. Associated with a composite primary dimensionless scalar $\mathbb{N}$ is the $\mathcal{O}(2)$ multiplet

$$
\mathbb{G}^{(2)}=v_{i} v_{j} \mathbb{G}^{i j} \equiv G^{(2)}[\mathbb{N}]=-\frac{\mathrm{i}}{8} \Delta^{(4)} \nabla^{(-2)} \mathbb{N} \quad \Longleftrightarrow \quad \mathbb{G}^{i j}=-\frac{3 \mathrm{i}}{40} \Delta^{i j k l} \nabla_{k l} \mathbb{N} .
$$

By construction, $\mathbb{G}^{i j}$ obeys the constraint (6.57). An example is provided by

$$
\mathbb{N}=\left(\frac{W^{\hat{\alpha} \hat{\beta}} W_{\hat{\alpha} \hat{\beta}}}{G^{2 / 3}}\right)^{n}
$$

for a positive integer $n$. Here $W_{\hat{\alpha} \hat{\beta}}$ is the super Weyl tensor.

It is also possible to generate composite $\mathcal{O}(4+n)$ multiplets by making use of the prepotential construction (7.27), for any non-negative integer $n$. As an example, consider the case of an even integer $n=2 m$. Given a composite $\mathcal{O}(4+2 m)$ multiplet, we can introduce a composite tropical multiplet of the form (14.2) and then make use of the latter to generate the composite vector multiplet field strength (14.1).

As concerns the component approaches [14-23], there is essentially only one regular procedure (the vector-tensor embedding) to generate composite primary multiplets. It is defined as follows: given a composite vector multiplet field strength $\mathbb{W}$ constrained by (7.13), the following superfield

$$
\mathbb{H}_{\mathrm{VM}}^{i j} \equiv H_{\mathrm{VM}}^{i j}[\mathbb{W}]=\mathrm{i}\left(\nabla^{\hat{\alpha}(i} \mathbb{W}\right) \nabla_{\hat{\alpha}}^{j)} \mathbb{W}+\frac{\mathrm{i}}{2} \mathbb{W} \nabla^{\hat{\alpha}(i} \nabla_{\hat{\alpha}}^{j)} \mathbb{W}
$$

is a composite $\mathcal{O}(2)$ multiplet.

In addition, there exists the composite $\mathcal{O}(2)$ multiplet constructed by Hanaki, Ohashi and Tachikawa [29] and associated with the Weyl multiplet. ${ }^{34}$ In superspace, it is given in terms of the super Weyl tensor as in eq. (2.41).

We are in a position to generate supersymmetric invariants given primary composite multiplets. If the theory under consideration involves a dynamical vector multiplet, which

\footnotetext{
${ }^{34}$ This $\mathcal{O}(2)$ multiplet was denoted $L^{i j}\left[\mathbf{W}^{2}\right]$ in $[29]$.
} 
is described by a tropical prepotential $V(v)$, and also possesses a composite $\mathcal{O}(2)$ multiplet $\mathbb{G}^{(2)}$, a supersymmetric BF invariant is generated by the Lagrangian

$$
\mathcal{L}_{\mathbb{G}}^{(2)}=V \mathbb{G}^{(2)} .
$$

If the theory involves a dynamical $\mathcal{O}(2)$ multiplet, which is described by a prepotential $\Omega$, and possesses a composite vector multiplet field strength $\mathbb{W}$, then we are able to construct a supersymmetric invariant of the type (7.23) with the Lagrangian

$$
\mathcal{L}_{\mathbb{W}}=\Omega \mathbb{W} .
$$

More generally, the action principles (5.35) and (7.23) provide universal procedures to generate supersymmetric invariants. For instance, supersymmetric $R^{4+2 n}$ terms may be realized as full superspace invariants (7.23) with

$$
\mathcal{L}=\frac{\left(W^{\hat{\alpha} \hat{\beta}} W_{\hat{\alpha} \hat{\beta}}\right)^{2}}{G}\left(\frac{W[\mathbb{V}]}{G^{1 / 3}}\right)^{n}, \quad \mathbb{V}:=\frac{H_{\mathrm{Weyl}}^{(2)}}{G^{(2)}}, \quad n=0,1, \ldots,
$$

where $W[\mathbb{V}]$ is defined by (14.1).

\subsection{Composite vector multiplets}

In this subsection we consider several examples of applying the rule (14.1) to generate composite vector multiplets. Our results are inspired by the four-dimensional analysis in [69]. Below we denote $\mathbb{W}_{\text {tropical }}$ simply as $\mathbb{W}$.

Our first example is

$$
\mathbb{V}=\log \left(\frac{G^{(2)}}{i \Upsilon^{(1)} \breve{\Upsilon}^{(1)}}\right)
$$

where $\Upsilon^{(1)}$ is a weight-one arctic multiplet. The corresponding composite vector multiplet (14.1) has already appeared in (12.8). It constitutes the equation of motion for the theory of a single $\mathcal{O}(2)$ multiplet coupled to conformal supergravity. Evaluating the covariant derivatives gives

$$
\mathbb{W}=-\frac{\mathrm{i}}{16 \pi} \oint(v, \mathrm{~d} v)\left(\frac{\nabla^{(-2)} G^{(2)}}{G^{(2)}}-\frac{\left(\nabla^{(-1) \hat{\alpha}} G^{(2)}\right) \nabla_{\hat{\alpha}}^{(-1)} G^{(2)}}{\left(G^{(2)}\right)^{2}}\right) .
$$

The contour integral can be explicitly evaluated. To do so we make use of the identities

$$
\begin{aligned}
& \nabla_{\hat{\alpha}}^{(-1)} G^{(2)}=2 \varphi_{\hat{\alpha}}^{(1)}=2 \varphi_{\hat{\alpha}}^{i} v_{i}, \\
& \nabla^{(-2)} G^{(2)}=-4 \mathrm{i} F,
\end{aligned}
$$

where we have introduced the descendant superfields (8.8). Then applying the integration identities of [69], we obtain

$$
\begin{aligned}
\mathbb{W} & =-\frac{1}{4 \pi} \oint_{C}(v, \mathrm{~d} v)\left(\frac{F}{G^{(2)}}-\mathrm{i} \frac{\varphi^{(1) \hat{\alpha}} \varphi_{\hat{\alpha}}^{(1)}}{G^{(2)}}\right)=\frac{1}{4 G} F-\frac{\mathrm{i}}{8 G^{3}} \varphi^{\hat{\alpha} i} \varphi_{\hat{\alpha}}^{j} G_{i j} \\
& =\frac{\mathrm{i}}{48 G} \nabla^{\hat{\alpha} i} \nabla_{\hat{\alpha}}^{j} G_{i j}-\frac{\mathrm{i}}{72 G^{3}} G_{i j}\left(\nabla_{k}^{\hat{\alpha}} G^{i k}\right) \nabla_{\hat{\alpha} l} G^{j l} .
\end{aligned}
$$


From the $S$-supersymmetry transformations of $\varphi_{\hat{\alpha}}^{i}$ and $F$,

$$
S_{\hat{\alpha}}^{i} \varphi_{\hat{\beta}}^{j}=-6 \varepsilon_{\hat{\alpha} \hat{\beta}} G^{i j}, \quad S_{\hat{\alpha}}^{i} F=6 \mathrm{i} \varphi_{\hat{\alpha}}^{i},
$$

it is straightforward to explicitly check that $\mathbb{W}$ is primary.

It is an instructive exercise to show that the composite vector multiplet (14.14) can also be rewritten in the following compact form

$$
\mathbb{W}=\frac{\mathrm{i}}{16} G \nabla^{\hat{\alpha} i} \nabla_{\hat{\alpha}}^{j}\left(\frac{G_{i j}}{G^{2}}\right) .
$$

This expression resembles the one in four dimensions [69]. The vector multiplet (14.16) is actually well known. At the component level it was first derived by Zucker [89], using a brute force approach, as an extension of the construction for the improved $\mathcal{N}=2$ tensor multiplet in four dimensions [81].

As another example, we consider a composite tropical prepotential of the form

$$
\mathbb{V}_{n}=\frac{H^{(2 n)}}{\left[G^{(2)}\right]^{n}}, \quad n=1,2, \ldots
$$

where $H^{(2 n)}$ is an arbitrary $\mathcal{O}(2 n)$ multiplet. The corresponding composite vector multiplet

$$
\mathbb{W}_{n}=-\frac{\mathrm{i}}{16 \pi} \oint_{C}(v, \mathrm{~d} v) \nabla^{(-2)}\left(\frac{H^{(2 n)}}{\left(G^{(2)}\right)^{n}}\right)
$$

can be computed in complete analogy with the $4 \mathrm{D} \mathcal{N}=2$ analysis in [69]. Evaluating the covariant derivatives gives

$$
\begin{aligned}
\mathbb{W}_{n}=-\frac{\mathrm{i}}{16 \pi} & \oint_{C}(v, \mathrm{~d} v)\left(\frac{2 n-1}{2 n+1} \frac{h^{(2 n-2)}}{\left(G^{(2)}\right)^{n}}-\frac{8 n^{2}}{2 n+1} \frac{\Psi^{(2 n-1)} \varphi^{(1)}}{\left(G^{(2)}\right)^{n+1}}+4 n \mathrm{i} H^{(2 n)} \frac{F}{\left(G^{(2)}\right)^{n+1}}\right. \\
& \left.+4 n(n+1) H^{(2 n)} \frac{\varphi^{(1)} \varphi^{(1)}}{\left(G^{(2)}\right)^{n+2}}\right)
\end{aligned}
$$

where we made use of the identities (14.13) and

$$
\begin{aligned}
\nabla_{\hat{\alpha}}^{-} H^{(2 n)} & =\frac{2 n}{2 n+1} \Psi_{\hat{\alpha}}^{(2 n-1)}, \\
\nabla^{(-2)} H^{(2 n)} & =\frac{2 n-1}{2 n+1} h^{2 n-2},
\end{aligned}
$$

with

$$
\begin{aligned}
\Psi_{\hat{\alpha}}^{(2 n-1)} & =\nabla_{\hat{\alpha} k} H^{k i_{1} \cdots i_{2 n-1}} v_{i_{1}} \cdots v_{i_{2 n-1}}, \\
h^{(2 n-2)} & =\nabla_{k l} H^{k l i_{1} \cdots i_{2 n-2}} v_{i_{1}} \cdots v_{i_{2 n-2}} .
\end{aligned}
$$

Making use of the integration results of [69] gives

$$
\begin{aligned}
\mathbb{W}_{n}= & \frac{\mathrm{i}(2 n) !}{2^{2 n+2}(n !)^{2}}\left(\frac{n}{2(2 n+1)} \frac{h^{i_{1} \cdots i_{2 n-2}} G_{\left(i_{1} i_{2}\right.} \cdots G_{\left.i_{2 n-3} i_{2 n-2}\right)}}{G^{2 n-1}}\right. \\
& -\frac{2 n^{2}}{2 n+1} \frac{\Psi^{i_{1} \cdots i_{2 n-1}} \varphi^{i_{2 n}} G_{\left(i_{1} i_{2}\right.} \cdots G_{\left.i_{2 n-1} i_{2 n}\right)}}{G^{2 n+1}}
\end{aligned}
$$




$$
\begin{aligned}
& +\mathrm{i} n \frac{F h^{i_{1} \cdots i_{2 n}} G_{\left(i_{1} i_{2}\right.} \cdots G_{\left.i_{2 n-1} i_{2 n}\right)}}{G^{2 n+1}} \\
& \left.+n(2 n+1) \frac{h^{i_{1} \cdots i_{2 n}} \varphi^{i_{2 n+1}} \varphi^{i_{2 n+2}} G_{\left(i_{1} i_{2}\right.} \cdots G_{\left.i_{2 n+1} i_{2 n+2}\right)}}{G^{n+3}}\right) .
\end{aligned}
$$

It turns out the above complex expression may be cast in the following simpler form

$$
\mathbb{W}_{n}=\frac{\mathrm{i}(2 n) !}{2^{2 n+3}(n+1) !(n-1) !} G \nabla_{i j} \mathcal{R}_{n}{ }^{i j},
$$

where

$$
\mathcal{R}_{n}^{i j}=\left(\delta_{k}^{i} \delta_{l}^{j}-\frac{1}{2 G^{2}} G^{i j} G_{k l}\right) \frac{H^{k l i_{1} \cdots i_{2 n-2}} G_{\left(i_{1} i_{2}\right.} \cdots G_{\left.i_{2 n-3} i_{2 n-2}\right)}}{G^{(2 n)}} .
$$

The composite vector multiplets (14.24) are new for $n>1$. The choice $n=1$ is a special case in the family of composite tropical prepotentials of the form

$$
\mathbb{V}=\mathcal{F}\left(H_{A}^{(2)}\right), \quad A=1, \ldots, m,
$$

where $\mathcal{F}\left(z_{A}\right)$ is a homogeneous function of degree zero, $\mathcal{F}\left(\lambda z_{A}\right)=\mathcal{F}\left(z_{A}\right)$, and $H_{A}^{(2)}$ are $\mathcal{O}(2)$ multiplets, $A=1, \ldots, m$. The composite vector multiplet associated with (14.25) can be computed in complete analogy with the $4 \mathrm{D} \mathcal{N}=2$ analysis in [69] (the latter analysis was inspired by [90]).

\subsection{Ricci squared $\mathcal{O}(2)$ multiplet}

As discussed above, associated with the super Weyl tensor is the $\mathcal{O}(2)$ multiplet (2.41). In this subsection we discover one more $\mathcal{O}(2)$ multiplet associated with the supergravity dynamical variables. Our analysis is inspired by the construction of chiral invariants in 4D $\mathcal{N}=2$ supergravity presented in [50].

In section 7.1 we constructed the prepotential formulation for the $\mathcal{O}(2)$ multiplet such that the prepotential is a primary dimensionless real scalar $\Omega$. It turns out that this construction can be generalized by replacing $\Omega$ with $\log \Phi$ defined in terms of a primary nowhere vanishing real scalar $\Phi$ of dimension $q$ :

$$
S_{\hat{\alpha}}^{i} \log \Phi=0, \quad \mathbb{D} \log \Phi=q .
$$

Let us consider the superfield

$$
G^{(2)}[\log \Phi]=-\frac{\mathrm{i}}{8} \Delta^{(4)} \nabla^{(-2)} \log \Phi=-\frac{3 \mathrm{i}}{40} v_{i} v_{j} \Delta^{i j k l} \nabla_{k l} \log \Phi .
$$

It follows that $G^{(2)}[\log \Phi]$ is analytic, $\nabla_{\hat{\alpha}}^{(1)} G^{(2)}[\log \Phi]=0$, and of dimension 3. As demonstrated in section 7.1, the superfield $G^{(2)}:=G^{(2)}[\Omega]$ defined by $(7.5)$ is primary $S_{\hat{\alpha}}^{i} G^{(2)}=0$. We observe that exactly the same derivation holds for $G^{(2)}[\log \Phi]$. Indeed, in the case of $G^{(2)}$ we used the fact that $\mathbb{D} \Omega=0$. In computing $S_{\hat{\alpha}}^{i} G^{(2)}[\log \Phi]$, there may be extra terms due to the fact that $\mathbb{D} \log \Phi=q \neq 0$. But it can be checked that all these terms are 
actually annihilated by some operator acting on the constant $q$. Since $S_{\hat{\alpha}}^{i} G^{(2)}[\log \Phi]=0$, we conclude that $G^{(2)}[\log \Phi]$ is also an $\mathcal{O}^{(2)}$ multiplet.

The reason why $G^{(2)}[\log \Phi]$ is of interest can be made clear once we consider the degauged versions of (7.5) and (14.27). It is a straightforward, although tedious, exercise to apply the degauging procedure of section 3 in order to express (7.5) and (14.27) in $\mathrm{SU}(2)$ superspace. Let us denote by $\mathcal{G}^{(2)}=\mathfrak{O}_{6}^{(2)} \Omega=v_{i} v_{j} \mathfrak{O}_{6}^{i j} \Omega$ the degauged version of (7.5). Here the sixth-order differential operator $\mathfrak{O}_{6}^{i j}=\mathfrak{O}_{6}^{j i}$ is constructed only in terms of $\mathcal{D}_{\hat{A}}$, $M_{\hat{a} \hat{b}}, J^{i j}$ and the torsion tensors of $\mathrm{SU}(2)$ superspace. It can be obtained by iteratively degauging the six $\nabla$-derivatives while moving to the right the $S_{\hat{\alpha}}^{i}, K_{\hat{a}}$ and $\mathbb{D}$ operators to use $S_{\hat{\alpha}}^{i} \Omega=K_{\hat{a}} \Omega=\mathbb{D} \Omega=0$. For the scope of this paper we do not need the explicit expression for $\mathfrak{O}_{6}^{i j}$. Since $G^{(2)}$ is an $\mathcal{O}(2)$ multiplet, it holds by construction that $\mathcal{D}_{\hat{\alpha}}^{(1)} \mathfrak{O}_{6}^{(2)} \Omega=0$.

The result of degauging $G^{(2)}[\log \Phi]$, which we denote $\mathcal{G}^{(2)}[\log \Phi]$, is more interesting. A straightforward but somewhat lengthy calculation leads to the following relation

$$
\mathcal{G}^{(2)}[\log \Phi]=\mathfrak{O}_{6}^{(2)} \log \Phi-q H_{\text {Ric }}^{(2)}
$$

The superfield $H_{\text {Ric }}^{(2)}$ encodes all the contributions that arise from using $\mathbb{D} \log \Phi=q$ and is given by

$$
\begin{aligned}
& H_{\text {Ric }}^{(2)}=-\frac{\mathrm{i}}{128}\left\{\mathcal{D}^{(2)} \mathcal{D}^{(2)} \mathfrak{F}^{(-2) \hat{\alpha}}{ }_{\hat{\alpha}}-12 \mathcal{D}^{(2)}\left(\mathfrak{F}^{(0) \hat{\alpha}}{ }_{\hat{\alpha}} \mathfrak{F}^{(0) \hat{\beta}}{ }_{\hat{\beta}}\right)+12 \mathcal{D}^{(2)}\left(\mathfrak{F}^{(0) \hat{\alpha} \hat{\beta}} \mathfrak{F}_{\hat{\alpha} \hat{\beta}}^{(0)}\right)\right. \\
& +12\left(3\left(\mathcal{D}^{(1) \hat{\alpha}} \mathfrak{F}_{\hat{\alpha}}^{(0)[\hat{\beta}}\right) \varepsilon^{\hat{\gamma} \hat{\delta}]}+\mathrm{i}\left(\Gamma_{\hat{a}}\right)^{\hat{\beta} \hat{\gamma}} \mathfrak{F}^{(1) \hat{\delta} \hat{a}}\right)\left(\mathcal{D}_{\hat{\beta}}^{(1)} \mathfrak{F}_{\hat{\gamma} \hat{\delta}}^{(0)}-\mathrm{i}\left(\Gamma^{\hat{b}}\right)_{\hat{\gamma} \hat{\delta}} \mathfrak{F}_{\hat{\beta} \hat{b}}^{(1)}\right) \\
& -12\left(3 \mathfrak{F}^{(0) \hat{\alpha}[\hat{\beta}} \varepsilon^{\hat{\gamma} \hat{\delta}]}+\mathfrak{F}^{(0) \hat{\delta} \hat{\alpha}} \varepsilon^{\hat{\beta} \hat{\gamma}}\right) \mathcal{D}_{\hat{\alpha}}^{(1)}\left(\mathcal{D}_{\hat{\beta}}^{(1)} \mathfrak{F}_{\hat{\gamma} \hat{\delta}}^{(0)}-\mathrm{i}\left(\Gamma^{\hat{b}}\right)_{\hat{\gamma} \hat{\delta}} \mathfrak{F}_{\hat{\beta} \hat{b}}^{(1)}\right) \\
& +8 \mathcal{D}^{(2)}\left(\mathfrak{F}^{(2) \hat{\alpha}} \mathfrak{F}^{(-2) \hat{\beta}}{ }_{\hat{\beta}}\right)+4 \mathcal{D}^{(1) \hat{\alpha}}\left(\mathfrak{F}_{\hat{\alpha}}^{(2)} \mathcal{D}_{\hat{\beta}}^{(1)} \mathfrak{F}^{(-2) \hat{\gamma}} \hat{\gamma}-6 \mathrm{i}\left(\Gamma^{\hat{a}}\right)^{\hat{\beta} \hat{\gamma}} \mathfrak{F}_{\hat{\alpha} \hat{a}}^{(1)} \mathfrak{F}_{\hat{\beta} \hat{\gamma}}^{(0)}\right) \\
& +16 \mathfrak{F}^{(2) \hat{\alpha} \hat{\beta}}\left(-2 \mathfrak{F}_{\hat{\alpha} \hat{\beta}}^{(2)} \mathfrak{F}^{(-2) \hat{\gamma}} \hat{\gamma}+6 \mathfrak{F}_{\hat{\alpha} \hat{\beta}}^{(0)} \mathfrak{F}^{(0) \hat{\gamma}} \hat{\gamma}-3 \mathfrak{F}_{\hat{\alpha}}^{(0) \hat{\gamma}} \mathfrak{F}_{\hat{\beta} \hat{\gamma}}^{(0)}\right. \\
& \left.+3 \mathfrak{F}^{(0) \hat{\gamma}} \mathfrak{F}_{\hat{\gamma} \hat{\beta}}^{(0)}+12 \mathfrak{F}^{(0)}{ }_{\hat{\alpha}} \mathfrak{F}_{\hat{\gamma} \hat{\beta}}^{(0)}\right) \\
& \left.+16 \mathfrak{F}^{(2) \hat{\alpha}}{ }_{\hat{\alpha}}\left(2 \mathfrak{F}^{(2) \hat{\beta}}{ }_{\hat{\beta}} \mathfrak{F}^{(-2) \hat{\gamma}}{ }_{\hat{\gamma}}+3 \mathfrak{F}^{(0) \hat{\beta} \hat{\gamma}} \mathfrak{F}_{\hat{\beta} \hat{\gamma}}^{(0)}-3 \mathfrak{F}^{(0) \hat{\beta}}{ }_{\hat{\beta}} \mathfrak{F}^{(0) \hat{\gamma}} \hat{\gamma}\right)\right\} \text {. }
\end{aligned}
$$

Here we have introduced the following superfields:

$$
\begin{aligned}
& \mathfrak{F}_{\hat{\alpha} \hat{\beta}}^{(2)}:=v_{i} v_{j} \mathfrak{F}_{\hat{\alpha} \hat{\beta}}^{i j}, \quad \mathfrak{F}_{\hat{\alpha} \hat{\beta}}^{(0)}:=\frac{v_{i} u_{j}}{(v, u)} \mathfrak{F}_{\hat{\alpha} \hat{\beta}}^{i j}, \quad \mathfrak{F}_{\hat{\alpha} \hat{\beta}}^{(-2)}:=\frac{u_{i} u_{j}}{(v, u)^{2}} \mathfrak{F}_{\hat{\alpha} \hat{\beta}}^{i j}, \\
& \mathcal{F}_{\hat{\alpha} \hat{\beta} \hat{\gamma}}^{(1)}:=\mathcal{D}_{\hat{\alpha}}^{(1)} \mathfrak{F}_{\hat{\beta} \hat{\gamma}}^{(0)}-\mathrm{i}\left(\Gamma^{\hat{b}}\right)_{\hat{\beta} \hat{\gamma}} \mathfrak{F}_{\hat{\alpha} \hat{b}}^{(1)} .
\end{aligned}
$$

What is remarkable about (14.28) is that by construction $H_{\text {Ric }}^{(2)}$ is a composite $\mathcal{O}(2)$ multiplet $^{35}$ constructed only in terms of the curvature tensors of $\mathrm{SU}(2)$ superspace; it is completely independent of $\log \Phi$. As will be discussed in the next two subsections, $\mathcal{G}^{(2)}[\log \Phi]$ gives rise to a supersymmetric extension of the Ricci squared action.

\footnotetext{
${ }^{35}$ It should be pointed out that $H_{\mathrm{Ric}}^{(2)}$ is a non-primary $\mathcal{O}(2)$ multiplet, since its super Weyl transformation law is inhomogeneous.
} 
By construction, $H_{\text {Ric }}^{(2)}$ is independent of $u_{i}$ and can be represented in the form $H_{\text {Ric }}^{(2)}=$ $v_{i} v_{j} H_{\text {Ric }}^{i j}$. From (14.29) we deduce

$$
\begin{aligned}
& H_{\text {Ric }}^{i j}=-\frac{\mathrm{i}}{128}\left\{\frac{3}{5} \mathcal{D}^{(i j} \mathcal{D}^{k l)} \mathfrak{F}_{k \hat{\alpha} l}^{\hat{\alpha}}-\frac{36}{5} \mathcal{D}^{(i j}\left(\mathfrak{F}_{\hat{\alpha} k}^{\hat{\alpha} k} \mathfrak{F}^{\hat{\beta} l)}{ }_{\hat{\beta} l}\right)+\frac{24}{5} \mathcal{D}^{(i j}\left(\mathfrak{F}^{\hat{\alpha} k l)} \mathfrak{F}_{k \hat{\beta} l}^{\hat{\beta}}\right)\right. \\
& +\frac{36}{5} \mathcal{D}^{(i j}\left(\mathfrak{F}_{k}^{\hat{\alpha} k \hat{\beta}} \mathfrak{F}_{\hat{\alpha} \hat{\beta} l}^{l)}\right)+6 \mathcal{D}^{\hat{\alpha}(i}\left(\frac{2}{5} \mathfrak{F}_{\hat{\alpha}}^{j} \hat{\beta} k \mathcal{D}_{\hat{\beta}}^{l)} \mathfrak{F}_{k}^{\hat{\gamma}} \hat{\gamma}-3 \mathrm{i}\left(\Gamma^{\hat{a}}\right)^{\hat{\beta} \hat{\gamma}} \mathfrak{F}_{\hat{\alpha} \hat{a}}^{j} \mathfrak{F}_{\hat{\beta}}^{k)} \hat{\gamma} k\right) \\
& +27\left(\mathcal{D}^{\hat{\alpha}(i} \mathfrak{F}_{\hat{\alpha} k}^{j[\hat{\beta}}\right) \varepsilon^{\hat{\gamma} \hat{\delta}]}\left(\frac{4}{5} \mathcal{D}_{\hat{\beta}}^{k} \mathfrak{F}_{\hat{\gamma} \hat{\delta} l}^{l}-\mathrm{i} \mathfrak{F}_{\hat{\beta} \hat{b}}^{k)}\left(\Gamma^{\hat{b}}\right)_{\hat{\gamma} \hat{\delta}}\right) \\
& +9\left(3 \mathfrak{F}_{k}^{\hat{\alpha}(i[\hat{\beta}} \varepsilon^{\hat{\gamma} \hat{\delta}]}+\mathfrak{F}_{k}^{\hat{\delta}\left(i \hat{\alpha} \varepsilon^{\hat{\beta} \hat{\gamma}}\right.}\right)\left(\mathrm{i} \mathcal{D}_{\hat{\alpha}}^{j} \mathfrak{F}_{\hat{\beta} \hat{b}}^{k)}\left(\Gamma^{\hat{b}}\right)_{\hat{\gamma} \hat{\delta}}-\frac{4}{5} \mathcal{D}_{\hat{\alpha}}^{j} \mathcal{D}_{\hat{\beta}}^{k} \mathfrak{F}_{\hat{\gamma} \hat{\delta} l}^{l)}\right) \\
& +9 \mathrm{i}\left(\Gamma_{\hat{a}}\right)^{\hat{\beta} \hat{\gamma}} \mathfrak{F}^{\hat{\delta}(i \hat{a}} \mathcal{D}_{\hat{\beta}}^{j} \mathfrak{F}_{\hat{\gamma} \hat{\delta} k}^{k)}-12\left(\Gamma_{\hat{a}}\right)_{\hat{\beta}}^{\hat{\gamma}}\left(\Gamma_{\hat{b}}\right)_{\hat{\gamma} \hat{\delta}} \mathfrak{F}^{\hat{\delta}(i a \hat{a}} \mathfrak{F}^{\hat{j} j) \hat{b}} \\
& +\frac{48}{5} \mathfrak{F}^{\hat{\alpha}(i \hat{\beta} j}\left(6 \mathfrak{F}_{\hat{\alpha} \hat{\beta} k}^{k} \mathfrak{F}_{\hat{\gamma} l)}^{\hat{\gamma} l)}-2 \mathfrak{F}_{\hat{\alpha} \hat{\beta}}^{k l)} \mathfrak{F}_{k}^{\hat{\gamma}}{ }_{\hat{\gamma} l}-3 \mathfrak{F}_{\hat{\alpha} k}^{k} \hat{F}_{\hat{\beta}}^{l)} \hat{\gamma} l+3 \mathfrak{F}_{\hat{\alpha} k}^{k} \mathfrak{F}_{\hat{\gamma} \hat{\beta} l}^{l)}+12 \mathfrak{F}_{\hat{\alpha} k}^{k} \mathfrak{F}_{\hat{\gamma} \hat{\beta} l}^{l)}\right) \\
& \left.+\frac{48}{5} \mathfrak{F}_{\hat{\alpha}}^{\hat{\alpha}(i j}\left(2 \mathfrak{F}_{\hat{\beta}}^{\hat{\beta} k l)} \mathfrak{F}_{k}^{\hat{\gamma}}{ }_{\hat{\gamma} l}+3 \mathfrak{F}_{k}^{\hat{\beta} k \hat{\gamma}} \mathfrak{F}_{\hat{\beta}}^{l)} \hat{\gamma} l-3 \mathfrak{F}^{\hat{\beta} k}{ }_{\hat{\beta} k} \mathfrak{F}^{\hat{F} l)}{ }_{\hat{\gamma} l}\right)\right\} \text {. }
\end{aligned}
$$

On the other hand, the condition that the expression (14.29) is independent of $u_{i}$ gives the constraints

$$
\begin{aligned}
& 0=\mathcal{D}^{(i j} \mathcal{D}^{k l} \mathfrak{F}_{\hat{\alpha}}^{\hat{\alpha} p q)}-4 \mathcal{D}^{(i j}\left(\mathfrak{F}_{\hat{\alpha}}^{\hat{\alpha} k l} \mathfrak{F}_{\hat{\beta}}^{\hat{\beta} p q)}\right)+12 \mathcal{D}^{(i j}\left(\mathfrak{F}^{\hat{k} k \hat{\beta} l} \mathfrak{F}_{\hat{\alpha} \hat{\beta}}^{p q)}\right) \\
& +4 \mathcal{D}^{\hat{\alpha}(i}\left(\mathfrak{F}_{\hat{\alpha}}^{j \hat{\beta} k} \mathcal{D}_{\hat{\beta}}^{l} \mathfrak{F}_{\hat{\gamma}}^{p q)}\right)+36\left(\mathcal{D}^{\hat{\alpha}(i} \mathfrak{F}_{\hat{\alpha}}^{j[\hat{\beta} k}\right) \varepsilon^{\hat{\gamma} \hat{\delta}]} \mathcal{D}_{\hat{\beta}}^{l} \mathfrak{F}_{\hat{\gamma} \hat{\delta}}^{p q)} \\
& -12\left(3 \mathfrak{F}^{\hat{\alpha}(i[\hat{\beta} j} \varepsilon^{\hat{\gamma} \hat{\delta}]}+\mathfrak{F}^{\hat{\delta}(i \hat{\alpha} j} \varepsilon^{\hat{\beta} \hat{\gamma}}\right) \mathcal{D}_{\hat{\alpha}}^{k} \mathcal{D}_{\hat{\beta}}^{l} \mathfrak{F}_{\hat{\gamma} \hat{\delta}}^{p q)} \\
& +16\left(7 \mathfrak{F}^{\hat{\alpha}(i \hat{\beta} j} \mathfrak{F}_{\hat{\alpha} \hat{\beta}}^{k l} \mathfrak{F}_{\hat{\gamma}}^{\hat{\gamma} p q)}-\mathfrak{F}_{\hat{\alpha}}^{\hat{\alpha}(i j} \mathfrak{F}_{\hat{\beta}}^{\hat{\beta} k l} \mathfrak{F}_{\hat{\gamma}}^{\hat{\gamma} p q)}+12 \mathfrak{F}^{\hat{\alpha}(i \hat{\beta} j} \mathfrak{F}_{\hat{\alpha}}^{k \hat{\gamma} l} \mathfrak{F}_{\hat{\gamma} \hat{\beta}}^{p q)}\right)
\end{aligned}
$$

and

$$
\begin{aligned}
& 0=\frac{5}{3} \mathcal{D}^{(i j} \mathcal{D}^{k l} \mathfrak{F}_{\hat{\alpha} p}^{\hat{\alpha} p)}-\frac{20}{3} \mathcal{D}^{(i j}\left(\mathfrak{F}_{\hat{\alpha}}^{\hat{\alpha} k l} \mathfrak{F}_{\hat{\beta} p}^{\hat{\beta} p)}\right)+20 \mathcal{D}^{(i j}\left(\mathfrak{F}^{\hat{\alpha} k \hat{\beta} l} \mathfrak{F}_{\hat{\alpha} \hat{\beta} p}^{p)}\right) \\
& +4 \mathcal{D}^{\hat{\alpha}(i}\left(\frac{5}{3} \mathfrak{F}_{\hat{\alpha}}^{j \hat{\beta} k} \mathcal{D}_{\hat{\beta}}^{l} \mathfrak{F}^{\hat{\gamma} p)} \hat{\gamma} p-6 \mathrm{i}\left(\Gamma^{\hat{a}}\right)^{\hat{\beta} \hat{\gamma}} \mathfrak{F}_{\hat{\alpha} \hat{a}}^{j} \mathfrak{F}_{\hat{\beta} \hat{\gamma}}^{k l)}\right) \\
& +6\left(\mathcal{D}^{\hat{\alpha}(i} \mathfrak{F}_{\hat{\alpha}}^{j[\hat{\beta} k}\right) \varepsilon^{\hat{\gamma} \hat{\delta}]}\left(5 \mathcal{D}_{\hat{\beta}}^{l} \mathfrak{F}_{\hat{\gamma} \hat{\delta} p}^{p)}-6 \mathrm{i} \mathfrak{F}_{\hat{\beta} \hat{b}}^{l)}\left(\Gamma^{\hat{b}}\right)_{\hat{\gamma} \hat{\delta}}\right)+30\left(\mathcal{D}^{\hat{\alpha}(i} \mathfrak{F}_{\hat{\alpha} p}^{j[\hat{\beta}}\right) \varepsilon^{\hat{\gamma} \hat{\delta}]} \mathcal{D}_{\hat{\beta}}^{k} \mathfrak{F}_{\hat{\gamma} \hat{\delta}}^{l p)} \\
& +2\left(3 \mathfrak{F}^{\hat{\alpha}(i[\hat{\beta} j} \varepsilon^{\hat{\gamma} \hat{\delta}]}+\mathfrak{F}^{\hat{\delta}(i \hat{\alpha} j} \varepsilon^{\hat{\beta} \hat{\gamma}}\right)\left(6 \mathrm{i} \mathcal{D}_{\hat{\alpha}}^{k} \mathfrak{F}_{\hat{\beta} \hat{b}}^{l)}\left(\Gamma^{\hat{b}}\right)_{\hat{\gamma} \hat{\delta}}-5 \mathcal{D}_{\hat{\alpha}}^{k} \mathcal{D}_{\hat{\beta}}^{l} \mathfrak{F}_{\hat{\gamma} \hat{\delta} p}^{p)}\right) \\
& -10\left(3 \mathfrak{F}_{p}^{\hat{\alpha}(i[\hat{\beta}} \varepsilon^{\hat{\gamma} \hat{\delta}]}+\mathfrak{F}_{p}^{\hat{\delta}(i \hat{\alpha}} \varepsilon^{\hat{\beta} \hat{\gamma}}\right) \mathcal{D}_{\hat{\alpha}}^{j} \mathcal{D}_{\hat{\beta}}^{k} \mathfrak{F}_{\hat{\gamma} \hat{\delta}}^{l p}+12 \mathrm{i}\left(\Gamma_{\hat{a}}\right)^{\hat{\beta} \hat{\gamma}} \mathfrak{F}^{\hat{\delta}(i \hat{a}} \mathcal{D}_{\hat{\beta}}^{j} \mathfrak{F}_{\hat{\gamma} \hat{\delta}}^{k l)} \\
& +\frac{80}{3} \mathfrak{F}^{\hat{\alpha}(i \hat{\beta} j}\left(\mathfrak{F}_{\hat{\alpha} \hat{\beta}}^{k l} \mathfrak{F}^{\hat{\gamma} p)} \hat{\gamma} p_{p}+3 \mathfrak{F}_{\hat{\alpha} \hat{\beta} p}^{k} \mathfrak{F}_{\hat{\gamma}}^{\hat{\gamma} l p)}-9 \mathfrak{F}_{\hat{\alpha}}^{k \hat{\gamma} l} \mathfrak{F}_{\hat{\beta}}^{p)} \hat{\gamma} p+3 \mathfrak{F}_{\hat{\alpha}}^{k} \hat{\gamma} l \mathfrak{F}_{\hat{\gamma} \hat{\beta} p}^{p)}\right) \\
& +\frac{80}{3} \mathfrak{F}_{\hat{\alpha}}^{\hat{\alpha}(i j}\left(3 \mathfrak{F}^{\hat{\beta} k \hat{\gamma} l} \mathfrak{F}_{\hat{\beta}}^{p)} \hat{\gamma} p-\mathfrak{F}_{\hat{\beta}}^{\hat{\beta} k l} \mathfrak{F}_{\hat{\gamma} p}^{\hat{\gamma} p)}\right),
\end{aligned}
$$

which have to be satisfied identically.

\subsection{Supersymmetric $R^{2}$ invariants}

Supersymmetric extensions of the $R^{2}$ terms may be realized using the BF action principle (8.1), in which the tropical prepotential corresponds to the vector multiplet compen- 
sator. There are three invariants associated with the Lagrangians

$$
\begin{aligned}
\mathcal{L}_{\text {Weyl }}^{(2)} & =V H_{\mathrm{Weyl}}^{(2)}, \\
\mathcal{L}_{\text {Ric }}^{(2)} & =-V G^{(2)}[\log W], \\
\mathcal{L}_{\text {scal }}^{(2)} & =V H_{\mathrm{VM}}^{(2)}[\mathbb{W}], \quad \mathbb{W}=\frac{\mathrm{i}}{16} G \nabla^{\hat{\alpha} i} \nabla_{\hat{\alpha}}^{j}\left(\frac{G_{i j}}{G^{2}}\right) .
\end{aligned}
$$

The supersymmetric invariants associated with (14.33a) and (14.33c) are known in the literature [29-32]. At the component level, they generate the Weyl tensor squared and scalar curvature squared terms, respectively. The invariant associated with (14.33b) is new. At the component level, it turns out to generate the Ricci tensor squared term. In order to achieve a better understanding of this invariant, it is useful to consider a special case when the vector multiplet compensator $W$ obeys the equation (13.2). As discussed in section 13.1, this case corresponds to the dilaton Weyl multiplet.

\subsection{The supersymmetric Ricci squared term and the dilaton Weyl multiplet}

When dealing with the vector multiplet compensator, it is often convenient to impose the gauge condition (3.2) which fixes the local special conformal symmetry and eliminates the dilatation connection entirely, thus leading us to $\mathrm{SU}(2)$ superspace. In addition, the local dilatation symmetry can also be fixed by making the gauge choice

$$
W=1 .
$$

We recall that the Bianchi identity for the vector multiplet (2.49) takes the following form in $\mathrm{SU}(2)$ superspace [26]

$$
\mathcal{D}_{\hat{\alpha}}^{(i} \mathcal{D}_{\hat{\beta}}^{j)} W-\frac{1}{4} \varepsilon_{\hat{\alpha} \hat{\beta}} \mathcal{D}^{\hat{\gamma}(i} \mathcal{D}_{\hat{\gamma}}^{j)} W=\frac{i}{2} C_{\hat{\alpha} \hat{\beta}}{ }^{i j} W
$$

Then choosing the gauge condition (14.34) gives

$$
C_{\hat{a}}^{i j}=0 .
$$

We also recall that the equation of motion for the vector multiplet (13.2) turns into (13.3) in $\mathrm{SU}(2)$ superspace. Then imposing the gauge condition (14.34) gives

$$
S^{i j}=0 \text {. }
$$

Under the conditions (14.36), the algebra of covariant derivatives in $\mathrm{SU}(2)$ superspace simplifies drastically. In particular, the anti-commutator of two spinor covariant derivatives becomes

$$
\begin{aligned}
\left\{\mathcal{D}_{\hat{\alpha}}^{i}, \mathcal{D}_{\hat{\beta}}^{j}\right\}= & -2 \mathrm{i} \varepsilon^{i j} \mathcal{D}_{\hat{\alpha} \hat{\beta}}-\mathrm{i} \varepsilon_{\hat{\alpha} \hat{\beta}} \varepsilon^{i j}\left(W^{\hat{c} \hat{d}}+Y^{\hat{c} \hat{d}}\right) M_{\hat{c} \hat{d}}+\frac{\mathrm{i}}{2} \varepsilon^{i j} \varepsilon^{\hat{a} \hat{b} \hat{c} \hat{d} \hat{e}}\left(\Gamma_{\hat{a}}\right)_{\hat{\alpha} \hat{\beta}} Y_{\hat{b} \hat{c}} M_{\hat{d} \hat{e}} \\
& -12 \mathrm{i} Y_{\hat{\alpha} \hat{\beta}} J^{i j}
\end{aligned}
$$

where $W_{\hat{a} \hat{b}}$ and $Y_{\hat{a} \hat{b}}$ satisfy the Bianchi identities

$$
\mathcal{D}_{\hat{\gamma}}^{k} W_{\hat{a} \hat{b}}=W_{\hat{a} \hat{b} \hat{\gamma}}^{k}-\frac{5}{2}\left(\Sigma_{\hat{a} \hat{b}}\right) \hat{\gamma} \mathcal{H}_{\hat{\delta}}^{k},
$$




$$
\mathcal{D}_{\hat{\gamma}}^{k} Y_{\hat{a} \hat{b}}=2\left(\Gamma_{[\hat{a}}\right)_{\hat{\gamma}}^{\hat{\delta}} \mathcal{Y}_{\hat{b}] \hat{\delta}}^{k}+\left(\Sigma_{\hat{a} \hat{b}}\right)_{\hat{\gamma}}^{\hat{\delta}} \mathcal{Y}_{\hat{\delta}}^{k}
$$

Using the Bianchi identities, at dimension 2 we find the relations

$$
\begin{aligned}
\mathcal{D}_{\hat{\alpha}}^{(i} \mathcal{D}_{\hat{\beta}}^{j)} W_{\hat{a} \hat{b}} & =\frac{5}{2} \varepsilon_{\hat{\alpha} \hat{\beta}}\left(\Sigma_{\hat{a} \hat{b}}\right)^{\hat{\gamma} \hat{\delta}} \mathcal{D}_{\hat{\gamma}}^{(i} \mathcal{Y}_{\hat{\delta}}^{j)}-\frac{5}{8} \varepsilon_{\hat{a} \hat{a} \hat{c} \hat{d} \hat{e}}\left(\Gamma^{\hat{e}}\right)_{\hat{\alpha} \hat{\beta}}\left(\Sigma^{\hat{c} \hat{d}}\right)^{\hat{\delta} \hat{\rho}} \mathcal{D}_{\hat{\delta}}^{(i} \mathcal{Y}_{\hat{\rho}}^{j)} \\
\mathcal{D}_{\hat{\alpha}}^{(i} \mathcal{D}_{\hat{\beta}}^{j)} Y_{\hat{a} \hat{b}} & =\frac{5}{4} \varepsilon_{\hat{\alpha} \hat{\beta}}\left(\Sigma_{\hat{a} \hat{b}}\right)^{\hat{\gamma} \hat{\delta}} \mathcal{D}_{\hat{\gamma}}^{(i} \mathcal{Y}_{\hat{\delta}}^{j)}+\frac{5}{8} \varepsilon_{\hat{a} \hat{b} \hat{c} \hat{d} \hat{e}}\left(\Gamma^{\hat{e}}\right)_{\hat{\alpha} \hat{\beta}}\left(\Sigma^{\hat{c} \hat{d}}\right)^{\hat{\gamma} \hat{\delta}} \mathcal{D}_{\hat{\gamma}}^{(i} \mathcal{Y}_{\hat{\delta}}^{j)}
\end{aligned}
$$

Furthermore, at dimension $5 / 2$ we derive

$$
\mathcal{D}_{\hat{\alpha}}^{(i} \mathcal{D}^{j k)} Y_{\hat{a} \hat{b}}=0, \quad \mathcal{D}_{\hat{\alpha}}^{(i} \mathcal{D}^{j k)} W_{\hat{a} \hat{b}}=0 .
$$

It can also be seen that the bivector $X_{\hat{a} \hat{b}}:=Y_{\hat{a} \hat{b}}+W_{\hat{a} \hat{b}}$ satisfies

$$
\mathcal{D}_{\hat{\alpha}}^{(i} \mathcal{D}_{\hat{\beta}}^{j)} X_{\hat{a} \hat{b}}=\frac{1}{4} \varepsilon_{\hat{\alpha} \hat{\beta}} \mathcal{D}^{i j} X_{\hat{a} \hat{b}} .
$$

This relation is reminiscent of the Bianchi identity for the vector multiplet, eq. (2.49). In the remainder of this section, we will refer to the superspace geometry described as dilaton $\mathrm{SU}(2)$ superspace.

In the dilaton $\mathrm{SU}(2)$ superspace, the expressions (14.28) and (14.29) for the $\mathcal{O}(2)$ multiplet on the right of (14.33b) proves to simplify drastically and takes the form:

$$
-\mathcal{G}^{(2)}[\log W]=H_{\mathrm{Ric}}^{(2)}=\frac{15 \mathrm{i}}{8}\left\{Y^{\hat{\alpha} \hat{\beta}} \mathcal{D}_{\hat{\alpha}}^{(1)} \mathcal{Y}_{\hat{\beta}}^{(1)}-\frac{2}{5} \mathcal{Y}^{(1) \hat{a} \hat{\alpha}} \mathcal{Y}_{\hat{a} \hat{\alpha}}^{(1)}-\frac{1}{2} \mathcal{Y}^{(1) \hat{\alpha}} \mathcal{Y}_{\hat{\alpha}}^{(1)}\right\}
$$

It is now easy to check that the constraints (14.32) are identically satisfied. Now we are going to show that $H_{\text {Ric }}^{(2)}$ can be represented as a linear combination of two different $\mathcal{O}(2)$ multiplets.

First of all, let us consider the Weyl squared $\mathcal{O}(2)$ multiplet (2.41). In the dilaton $\mathrm{SU}(2)$ superspace it may be rewritten as

$$
H_{\mathrm{Weyl}}^{(2)}=-\frac{15 \mathrm{i}}{4}\left\{W^{\hat{\alpha} \hat{\beta}} \mathcal{D}_{\hat{\alpha}}^{(1)} \mathcal{Y}_{\hat{\beta}}^{(1)}+\frac{1}{15} W^{(1) \hat{a} \hat{b} \hat{\alpha}} W_{\hat{a} \hat{b} \hat{\alpha}}^{(1)}+\frac{5}{4} \mathcal{Y}^{(1) \hat{\alpha}} \mathcal{Y}_{\hat{\alpha}}^{(1)}\right\}
$$

For the dilaton Weyl multiplet, the BF Lagrangian (14.33a) generates a supersymmetric extension of the $\left(C_{\hat{a} \hat{b} \hat{c} \hat{d}}\right)^{2}+\frac{1}{6} R^{2}$ Lagrangian of $[29,31,32]$.

A remarkable feature of the dilaton $\mathrm{SU}(2)$ superspace is that the relations (14.40) and (14.41) imply the existence of one more $\mathcal{O}(2)$ multiplet. It is

$$
\begin{aligned}
H_{\text {Riem }}^{(2)} & :=\frac{\mathrm{i}}{4}\left\{X^{\hat{a} \hat{b}} \mathcal{D}^{(2)} X_{\hat{a} \hat{b}}+2\left(\mathcal{D}^{(1) \hat{\alpha}} X^{\hat{a} \hat{b}}\right) \mathcal{D}_{\hat{\alpha}}^{(1)} X_{\hat{a} \hat{b}}\right\} \\
& =\frac{15 \mathrm{i}}{2}\left\{X^{\hat{\gamma} \hat{\delta}} \mathcal{D}_{\hat{\gamma}}^{(i} \mathcal{Y}_{\hat{\delta}}^{j)}+\frac{1}{15} W^{(1) \hat{a} \hat{b} \hat{\gamma}} W_{\hat{a} \hat{b} \hat{\gamma}}^{(1)}-\frac{2}{5} \mathcal{Y}^{(1) \hat{a} \hat{\alpha}} \mathcal{Y}_{\hat{a} \hat{\alpha}}^{(1)}+\frac{3}{4} \mathcal{Y}^{(1) \hat{\alpha}} \mathcal{Y}_{\hat{\alpha}}^{(1)}\right\} .
\end{aligned}
$$

One may check that $\mathcal{D}_{\hat{\alpha}}^{(1)} H_{\text {Riem }}^{(2)}=0$. The structure of $H_{\text {Riem }}^{(2)}$ resembles the composite $\mathcal{O}(2)$ multiplet built from a vector multiplet, eq. (14.7). It turns out that the $\mathcal{O}(2)$ multiplet (14.44) generates the supersymmetric extension of the Riemann squared term, $\left(R_{\hat{a} \hat{b} \hat{c} \hat{d}}\right)^{2}$, 
constructed in [30]. The construction of [30] was based on a map between the dilaton Weyl multiplet and the vector multiplet applied to the non-abelian Chern-Simons action.

From the relations $(14.42)-(14.44)$ we deduce

$$
-\mathcal{G}^{(2)}[\log W]=\frac{1}{2} H_{\mathrm{Weyl}}^{(2)}+\frac{1}{4} H_{\mathrm{Riem}}^{(2)} .
$$

The important point is that the construction of [30] and related works [31, 32] is defined only for the dilaton Weyl multiplet. Our Ricci squared $\mathcal{O}(2)$ multiplet $-G^{(2)}[\log W]$, eq. (14.27), and the corresponding supersymmetric invariant generated by (14.33b) makes use of the standard Weyl multiplet coupled to the off-shell vector multiplet compensator. Eq. (14.45) allows us to define $H_{\text {Riem }}^{(2)}$ for the standard Weyl multiplet coupled to the off-shell vector multiplet compensator:

$$
H_{\mathrm{Riem}}^{(2)}=-4\left(G^{(2)}[\log W]+\frac{1}{2} H_{\mathrm{Weyl}}^{(2)}\right)
$$

\section{Concluding remarks}

The conformal superspace formalism in five dimensions presented in this work combines the powerful features of the SU(2) superspace approach [26] and the superconformal tensor calculus [19-22]. Using this formalism we have reproduced practically all off-shell constructions derived so far. Most importantly, since the superspace setting offers more general off-shell multiplets than those employed in [19-22], we have developed novel tools to construct composite primary multiplets and, as a consequence, to generate new higher-order off-shell invariants in supergravity. In addition to full superspace integrals, we have introduced general techniques to build composite $\mathcal{O}(2)$ and vector multiplets, which in turn can be used in the universal BF action. One particular example is the Ricci squared $\mathcal{O}(2)$ multiplet constructed in section 14.3. ${ }^{36}$

Prior to this paper, the superconformal tensor calculus was used to construct supersymmetric completions of $R^{2}$ terms. Hanaki, Ohashi and Tachikawa [29] constructed the supersymmetric Weyl tensor squared term, while Ozkan and Pang [32] constructed the supersymmetric scalar curvature squared term. These invariants are generated by the Lagrangians (14.33a) and (14.33c) respectively. An important feature of these invariants is that they make use of the standard Weyl multiplet coupled to one or two conformal compensators, one of which is always the vector multiplet. Choosing the vector multiplet to be on-shell leads one to a formulation in the dilaton Weyl multiplet. As concerns a supersymmetric completion of the Riemann squared term, it was constructed by Bergshoeff, Rosseel and Sezgin [30] only in the dilaton Weyl multiplet realization. However, a description of the supersymmetric Riemann squared action in the standard Weyl multiplet was completely unknown. Our paper has solved this problem with the use of the $\mathcal{O}(2)$ multiplet $G^{(2)}[\log W]$, eq. (14.27), which describes a supersymmetric Ricci squared invariant

\footnotetext{
${ }^{36}$ The construction of the Ricci squared $\mathcal{O}(2)$ multiplet is analogous to that of the nonlinear kinetic multiplet presented in [50].
} 
using the Lagrangian (14.33b). This invariant completes the description of the supersymmetric $R^{2}$ invariants within the standard Weyl multiplet. In particular, the analogue of the supersymmetric Riemann squared action constructed in [30] is generated by (14.46). We hope to elaborate further the component structure of the action generated by the Lagrangian (14.33b) in another publication.

The main virtue of the $\mathrm{SU}(2)$ superspace approach [26] and its extension given in our paper is that it offers off-shell descriptions for the most general supergravity-matter systems. Here we briefly comment on such off-shell descriptions. In section 12, we discussed the two-derivative supergravity action, corresponding to an $\mathcal{O}(2)$ multiplet and an abelian vector multiplet compensator. It is easy to generalize this to include off-shell hypermultiplets. One takes the same approach as in four dimensions [83] and adds to the pure supergravity Lagrangian (12.1) a sigma model term ${ }^{37}$ resulting in

$$
\mathcal{L}_{\text {linear }}^{(2)}=\frac{1}{4} V H_{\mathrm{VM}}^{(2)}+G^{(2)} \ln \frac{G^{(2)}}{\mathrm{i}^{(1)} \breve{\Upsilon}^{(1)}}+\kappa V G^{(2)}+\frac{1}{2} G^{(2)} K(\Upsilon, \breve{\Upsilon})
$$

where $K(\Upsilon, \breve{\Upsilon})$ depends on $n$ weight-zero arctic multiplets $\Upsilon^{\mathcal{I}}$ and their smile-conjugate antarctic multiplets $\breve{\Upsilon} \overline{\mathcal{I}}$. Here $K\left(\varphi^{\mathcal{I}}, \bar{\varphi}^{\overline{\mathcal{I}}}\right)$ is chosen to be a real analytic function of $n$ ordinary complex variables $\varphi^{\mathcal{I}}$ and their conjugates. The action generated by the Lagrangian (15.1) proves to be invariant under the Kähler transformations

$$
K \rightarrow K+\Lambda(\Upsilon)+\bar{\Lambda}(\breve{\Upsilon})
$$

in accordance with eq. (7.17). This permits the identification of $K$ as the Kähler potential of a $2 n$-dimensional Kähler manifold $\mathcal{M}^{2 n}$.

The Lagrangian (15.1) is reminiscent of the general $4 \mathrm{D} \mathcal{N}=1$ new minimal supergravity-matter Lagrangian, which similarly involves a linear multiplet compensator coupled to a matter sector described by a Kähler potential, see [64] for a review. As in that situation, it is possible here to perform a duality transformation exchanging $G^{(2)}$ for a weight-one arctic multiplet $\Upsilon^{(1)}$ and its smile-conjugate antarctic $\breve{\Upsilon}^{(1)}$. The analogous consideration in the case of $4 \mathrm{D} \mathcal{N}=2$ supergravity was given in [83]. Following [83], the Lagrangian dual to (15.1) is

$$
\mathcal{L}_{\text {hyper }}^{(2)}=\frac{1}{4} V H_{\mathrm{VM}}^{(2)}-2 \mathrm{i} \breve{\Upsilon}^{(1)} \mathrm{e}^{-\kappa V-\frac{1}{2} K(\Upsilon, \breve{\Upsilon})} \Upsilon^{(1)}
$$

Here the compensator $\Upsilon^{(1)}$ is charged under the $\mathrm{U}(1)$ gauge group and transforms under the Kähler transformations (15.2) as $\Upsilon^{(1)} \rightarrow \mathrm{e}^{\Lambda / 2} \Upsilon^{(1)}$.

This supergravity-matter system may equivalently be described in terms of $(n+1)$ weight-one arctic multiplets $\Upsilon^{(1) I}$ and their conjugates $\breve{\Upsilon}^{(1) \bar{I}}$ defined by $\Upsilon^{(1) I}=\Upsilon^{(1)} \times$ $\left(1, \Upsilon^{\mathcal{I}}\right)$ for $I=0, \cdots, n$. The corresponding Lagrangian is

$$
\mathcal{L}_{\text {hyper }}^{(2)}=\frac{1}{4} V H_{\mathrm{VM}}^{(2)}-2 \mathrm{i} \mathrm{e}^{-\kappa V} \mathcal{K}\left(\Upsilon^{(1)}, \breve{\Upsilon}^{(1)}\right) .
$$

\footnotetext{
${ }^{37}$ The normalization of (15.1) is chosen so that in the super Weyl gauge $G=1$, it reproduces a canonically normalized sigma model.
} 
Here $\mathcal{K}\left(\Upsilon^{(1)}, \breve{\Upsilon}^{(1)}\right)$ obeys the homogeneity conditions

$$
\Upsilon^{(1) I} \frac{\partial}{\partial \Upsilon^{(1) I}} \mathcal{K}=\mathcal{K}, \quad \breve{\Upsilon}^{(1) \bar{I}} \frac{\partial}{\partial \breve{\Upsilon}^{(1) \bar{I}}} \mathcal{K}=\mathcal{K} .
$$

In addition, $\mathcal{K}\left(\varphi^{I}, \bar{\varphi}^{\bar{I}}\right)$ is required to be real as a function of $(n+1)$ ordinary complex variables $\varphi^{I}$ and their conjugates. Moreover, the action generated by the Lagrangian (15.4a) is invariant under the gauge transformations

$$
\delta V=\lambda+\breve{\lambda}, \quad \delta \Upsilon^{(1) I}=\kappa \lambda \Upsilon^{(1) I},
$$

with the gauge parameter $\lambda$ being an arbitrary weight-zero arctic multiplet.

The Lagrangian (15.1) and each of its dual versions, (15.3) and (15.4), actually describes a large class of $4 n$-dimensional quaternion-Kähler sigma models that admit a maximal $2 n$-dimensional Kähler submanifold with Kähler potential $K$ [91]. These sigma models also automatically possess a quaternionic $\mathrm{U}(1)$ isometry. To see this latter feature, one observes that the Lagrangian (15.1) describes a superconformal sigma model coupling the linear multiplet $G^{(2)}$ to the $n$ weight-zero polar multiplets. When the three-form in the linear multiplet is dualized, the resulting scalar manifold is a hyperkähler cone with a triholomorphic $\mathrm{U}(1)$ isometry. When $G^{(2)}$ is gauge-fixed, the $(4 n+4)$-dimensional hyperkähler cone becomes a $4 n$-dimensional quaternion-Kähler space, and the triholomorphic isometry descends to a quaternionic one. ${ }^{38}$

The most general $4 n$-dimensional quaternion-Kähler sigma model is described by a very similar supergravity-matter Lagrangian (for simplicity we switch off the cosmological constant)

$$
\mathcal{L}_{\text {hyper }}^{(2)}=\frac{1}{4} V H_{\mathrm{VM}}^{(2)}-2 \mathcal{F}\left(\Upsilon^{(1)}, \breve{\Upsilon}^{(1)}\right),
$$

where $\mathcal{F}\left(\Upsilon^{(1)}, \breve{\Upsilon}^{(1)}\right)$ obeys the homogeneity condition

$$
\left(\Upsilon^{(1) I} \frac{\partial}{\partial \Upsilon^{(1) I}}+\breve{\Upsilon}^{(1) \bar{I}} \frac{\partial}{\partial \breve{\Upsilon}^{(1) \bar{I}}}\right) \mathcal{F}=2 \mathcal{F}
$$

The dynamical system defined by eqs. (15.4a) and (15.4b) with $\kappa=0$ is a special case of the system under consideration. In the flat superspace limit, the Lagrangian $\mathcal{L}^{(2)}=$ $\mathcal{F}\left(\Upsilon^{(1)}, \breve{\Upsilon}^{(1)}\right)$ describes the most general superconformal sigma model, with its target space being an arbitrary hyperkähler cone. If the stronger homogeneity conditions (15.4b) hold, then the corresponding hyperkähler cone possesses a triholomorphic isometry, which is associated with the rigid U(1) symmetry of the superfield Lagrangian $\Upsilon^{(1) I} \rightarrow \mathrm{e}^{\mathrm{i} \varphi} \Upsilon^{(1) I}$, with $\varphi \in \mathbb{R}$. Similar issues have been discussed in the case of the $(3,0)$ supersymmetric sigma models in $\mathrm{AdS}_{3}$ [94].

The Lagrangian (15.6) can be generalized to include additional abelian vector multiplets in a straightforward way,

$$
\mathcal{L}^{(2)}=\frac{1}{4} C_{\mathrm{abc}} V^{\mathrm{a}} H^{(2) \mathrm{bc}}-2 \mathcal{F}\left(\Upsilon^{(1)}, \breve{\Upsilon}^{(1)}\right)
$$

\footnotetext{
${ }^{38}$ The link between triholomorphic isometries on the hyperkähler cone (or Swann bundle) and quaternionic isometries on the quaternion-Kähler space is known from the mathematics literature [92]. It was discussed in a physics context in [93].
} 


$$
H^{(2) \mathrm{ab}}:=\mathrm{i}\left(\nabla^{(1) \hat{\alpha}} W^{\mathrm{a}}\right) \nabla_{\hat{\alpha}}^{(1)} W^{\mathrm{b}}+\frac{\mathrm{i}}{2} W^{(\mathrm{a}} \nabla^{(1) \hat{\alpha}} \nabla_{\hat{\alpha}}^{(1)} W^{\mathrm{b})},
$$

for real constants $C_{\mathrm{abc}}=C_{(\mathrm{abc})}$, as is well-known from the component literature. The numerical factors chosen in front of the two terms in (15.8) ensure that the Weyl multiplet equation of motion and the canonical Weyl gauge are respectively given by

$$
C(W):=C_{\mathrm{abc}} W^{\mathrm{a}} W^{\mathrm{b}} W^{\mathrm{c}}=\mathbb{K}, \quad C(W)=1,
$$

where $\mathbb{K}$ is the hyperkähler potential constructed from $\mathcal{F}{ }^{39}$ The component reduction of the vector multiplet Lagrangian in (15.8) can be derived from the general result for the nonabelian vector multiplet action given in section 10 . The component reduction of the hypermultiplet sigma model can be carried out similarly to the $4 \mathrm{D} \mathcal{N}=2$ case worked out in [95].

The SU(2) superspace approach to $5 \mathrm{D}$ conformal supergravity coupled to general matter systems [26] has been extended to locally supersymmetric theories in diverse dimensions: $4 \mathrm{D} \mathcal{N}=2$ supergravity [47], 2D $\mathcal{N}=(4,4)$ supergravity [96, 97], 3D $\mathcal{N}=3$ and $\mathcal{N}=4$ supergravity theories [98], and $6 \mathrm{D} \mathcal{N}=(1,0)$ supergravity [99]. In four dimensions, $\mathcal{N}=2$ conformal superspace was formulated in [39], see also [55]. In three dimensions, $\mathcal{N}$-extended conformal superspace was described in [40]. Interesting open problems are to develop conformal superspace settings in other cases such as the $2 \mathrm{D} \mathcal{N}=(4,4)$ and $6 \mathrm{D} \mathcal{N}=(1,0)$ ones.

\section{Acknowledgments}

The work of DB was supported by ERC Advanced Grant No. 246974, "Supersymmetry: a window to non-perturbative physics" and by the European Commission Marie Curie International Incoming Fellowship grant no. PIIF-GA-2012-627976. The work of SMK and JN was supported by the Australian Research Council, project No. DP0103925. The work of GT-M and JN was supported by the Australian Research Council's Discovery Early Career Award (DECRA), project No. DE120101498. The work of SMK, JN and GT-M was also supported by the Australian Research Council project No. DP140103925.

\section{A Notation and conventions}

Throughout the paper we follow the 5D notation and conventions in [2]. We summarize them here and include a number of useful identities.

The $5 \mathrm{D}$ gamma-matrices $\Gamma_{\hat{a}}=\left(\Gamma_{a}, \Gamma_{5}\right)$, with $a=0,1,2,3$, are defined by

$$
\left\{\Gamma_{\hat{a}}, \Gamma_{\hat{b}}\right\}=-2 \eta_{\hat{a} \hat{b}} \mathbb{1}, \quad\left(\Gamma_{\hat{a}}\right)^{\dagger}=\Gamma_{0} \Gamma_{\hat{a}} \Gamma_{0},
$$

where the Minkowski metric is

$$
\eta_{\hat{a} \hat{b}}=\operatorname{diag}(-1,1,1,1,1) .
$$

\footnotetext{
${ }^{39}$ Our conventions for relating the hyperkähler potential to the Lagrangian $\mathcal{F}$ are the same as in [95]. There the potential was denoted $K$.
} 
We may choose a representation in which the gamma-matrices take the form $[62,64]$

$$
\left(\Gamma_{a}\right)_{\hat{\alpha}}^{\hat{\beta}}=\left(\begin{array}{cc}
0 & \left(\sigma_{a}\right)_{\alpha \dot{\beta}} \\
\left(\tilde{\sigma}_{a}\right)^{\dot{\alpha} \beta} & 0
\end{array}\right), \quad\left(\Gamma_{5}\right)_{\hat{\alpha}}^{\hat{\beta}}=\left(\begin{array}{cc}
-\mathrm{i} \delta_{\alpha}^{\beta} & 0 \\
0 & \mathrm{i} \delta_{\dot{\beta}}^{\dot{\alpha}}
\end{array}\right)
$$

and $\Gamma_{0} \Gamma_{1} \Gamma_{2} \Gamma_{3} \Gamma_{5}=\mathbb{1}$. The charge conjugation matrix, $C=\left(\varepsilon^{\hat{\alpha} \hat{\beta}}\right)$, and its inverse, $C^{-1}=$ $C^{\dagger}=\left(\varepsilon_{\hat{\alpha} \hat{\beta}}\right)$ are defined by

$$
C \Gamma_{\hat{a}} C^{-1}=\left(\Gamma_{\hat{a}}\right)^{\mathrm{T}}, \quad \varepsilon^{\hat{\alpha} \hat{\beta}}=\left(\begin{array}{cc}
\varepsilon^{\alpha \beta} & 0 \\
0 & -\varepsilon_{\dot{\alpha} \dot{\beta}}
\end{array}\right), \quad \varepsilon_{\hat{\alpha} \hat{\beta}}=\left(\begin{array}{cc}
\varepsilon_{\alpha \beta} & 0 \\
0 & -\varepsilon^{\dot{\alpha} \dot{\beta}}
\end{array}\right),
$$

where $\varepsilon^{\hat{\alpha} \hat{\beta}}$ and $\varepsilon_{\hat{\alpha} \hat{\beta}}$ are antisymmetric tensors which are used to raise and lower the fourcomponent spinor indices.

A Dirac spinor, $\Psi=\left(\Psi_{\hat{\alpha}}\right)$, and its Dirac conjugate, $\bar{\Psi}=\left(\bar{\Psi}^{\hat{\alpha}}\right)=\Psi^{\dagger} \Gamma_{0}$, decompose into two-component spinors as follows

$$
\Psi_{\hat{\alpha}}=\left(\begin{array}{c}
\psi_{\alpha} \\
\bar{\phi}^{\dot{\alpha}}
\end{array}\right), \quad \bar{\Psi}_{\hat{\alpha}}=\left(\phi^{\alpha}, \bar{\psi}_{\dot{\alpha}}\right) .
$$

One can combine $\bar{\Psi}^{\hat{\alpha}}=\left(\phi^{\alpha}, \bar{\psi}_{\dot{\alpha}}\right)$ and $\Psi^{\hat{\alpha}}=\varepsilon^{\hat{\alpha} \hat{\beta}} \Psi_{\hat{\beta}}=\left(\psi^{\alpha},-\bar{\phi}_{\dot{\alpha}}\right)$ into a SU(2) doublet,

$$
\Psi_{i}^{\hat{\alpha}}=\left(\Psi_{i}^{\alpha},-\bar{\Psi}_{\dot{\alpha} i}\right), \quad \overline{\left(\Psi_{i}^{\alpha}\right)}=\bar{\Psi}^{\dot{\alpha} i}, \quad i=\underline{1}, \underline{2},
$$

with $\Psi_{1}^{\alpha}=\phi^{\alpha}$ and $\Psi_{2}^{\alpha}=\psi^{\alpha}$. It is understood that the $\mathrm{SU}(2)$ indices are raised and lowered by $\varepsilon^{i j}$ and $\varepsilon_{i j}, \varepsilon^{\underline{12}}=\varepsilon_{\underline{21}}=1$, in the standard fashion: $\Psi^{\hat{\alpha} i}=\varepsilon^{i j} \Psi_{j}^{\hat{\alpha}}$. The Dirac spinor $\Psi^{i}=\left(\Psi_{\hat{\alpha}}^{i}\right)$ satisfies the pseudo-Majorana reality condition $\bar{\Psi}_{i}^{\mathrm{T}}=C \Psi_{i}$. This can be concisely written as

$$
\left(\Psi_{\hat{\alpha}}^{i}\right)^{*}=\Psi_{i}^{\hat{\alpha}} .
$$

In defining products of spinors, we occasionally suppress spinor indices. In such cases, the spinor indices should be understood as contracted from top left to bottom right; that is, given $\chi_{\hat{\alpha}}$ and $\Psi_{\hat{\alpha}}$, we define

$$
\chi \Psi:=\chi^{\hat{\alpha}} \Psi_{\hat{\alpha}}, \quad \chi \Gamma^{\hat{a}} \Psi:=\chi^{\hat{\alpha}}\left(\Gamma^{\hat{a}}\right)_{\hat{\alpha}}^{\hat{\beta}} \Psi_{\hat{\beta}}, \quad \chi \Gamma^{\hat{a}} \Gamma^{\hat{b}} \Psi:=\chi^{\hat{\alpha}}\left(\Gamma^{\hat{a}}\right)_{\hat{\alpha}}^{\hat{\beta}}\left(\Gamma^{\hat{b}}\right)_{\hat{\beta}}^{\hat{\gamma}} \Psi_{\hat{\gamma}},
$$

and so forth.

With the definition $\Sigma_{\hat{a} \hat{b}}=-\Sigma_{\hat{b} \hat{a}}=-\frac{1}{4}\left[\Gamma_{\hat{a}}, \Gamma_{\hat{b}}\right]$, the matrices $\left\{\mathbb{1}, \Gamma_{\hat{a}}, \Sigma_{\hat{a} \hat{b}}\right\}$ form a basis in the space of $4 \times 4$ matrices. The matrices $\varepsilon_{\hat{\alpha} \hat{\beta}}$ and $\left(\Gamma_{\hat{a}}\right)_{\hat{\alpha} \hat{\beta}}$ are antisymmetric (with $\left.\varepsilon^{\hat{\alpha} \hat{\beta}}\left(\Gamma_{\hat{a}}\right)_{\hat{\alpha} \hat{\beta}}=0\right)$, while the matrices $\left(\Sigma_{\hat{a} \hat{b}}\right)_{\hat{\alpha} \hat{\beta}}$ are symmetric.

It is useful to write explicitly the $4 \mathrm{D}$ reduction of these matrices

$$
\begin{aligned}
\left(\Gamma_{a}\right)_{\hat{\alpha} \hat{\beta}}=\left(\begin{array}{cc}
0 & -\left(\sigma_{a}\right)_{\alpha} \dot{\beta} \\
\left(\sigma_{a}\right)_{\beta} \dot{\alpha} & 0
\end{array}\right), & \left(\Gamma_{5}\right)_{\hat{\alpha} \hat{\beta}}=\left(\begin{array}{cc}
\mathrm{i} \varepsilon_{\alpha \beta} & 0 \\
0 & \mathrm{i} \varepsilon^{\dot{\alpha} \dot{\beta}}
\end{array}\right), \\
\left(\Sigma_{a b}\right)_{\hat{\alpha}}^{\hat{\beta}}=\left(\begin{array}{cc}
\left(\sigma_{a b}\right)_{\alpha}{ }^{\beta} & 0 \\
0 & \left(\tilde{\sigma}_{a b}\right)^{\dot{\alpha}}{ }_{\dot{\beta}}
\end{array}\right), & \left(\Sigma_{a 5}\right)_{\hat{\alpha}}^{\hat{\beta}}=\left(\begin{array}{cc}
0 & -\frac{\mathrm{i}}{2}\left(\sigma_{a}\right)_{\alpha \dot{\beta}} \\
\frac{\mathrm{i}}{2}\left(\tilde{\sigma}_{a}\right)^{\dot{\alpha} \beta} & 0
\end{array}\right),
\end{aligned}
$$




$$
\left(\Sigma_{a b}\right)_{\hat{\alpha} \hat{\beta}}=\left(\begin{array}{cc}
\left(\sigma_{a b}\right)_{\alpha \beta} & 0 \\
0 & -\left(\tilde{\sigma}_{a b}\right)^{\dot{\alpha} \dot{\beta}}
\end{array}\right), \quad\left(\Sigma_{a 5}\right)_{\hat{\alpha} \hat{\beta}}=\left(\begin{array}{cc}
0 & \frac{\mathrm{i}}{2}\left(\sigma_{a}\right)_{\alpha}^{\dot{\beta}} \\
\frac{\mathrm{i}}{2}\left(\sigma_{a}\right)_{\beta}{ }^{\dot{\alpha}} & 0
\end{array}\right)
$$

where $\left(\sigma_{a b}\right)_{\alpha}{ }^{\beta}=-\frac{1}{4}\left(\sigma_{a} \tilde{\sigma}_{b}-\sigma_{b} \tilde{\sigma}_{a}\right)_{\alpha}^{\beta}$ and $\left(\tilde{\sigma}_{a b}\right)_{\dot{\beta}}^{\dot{\alpha}}=-\frac{1}{4}\left(\tilde{\sigma}_{a} \sigma_{b}-\tilde{\sigma}_{b} \sigma_{a}\right)^{\dot{\alpha}} \dot{\beta}$.

A 5-vector $V^{\hat{a}}$ and an antisymmetric tensor $F^{\hat{a} \hat{b}}=-F^{\hat{b} \hat{a}}$ can be equivalently represented as the bi-spinors $V=V^{\hat{a}} \Gamma_{\hat{a}}$ and $F=\frac{1}{2} F^{\hat{a} \hat{b}} \Sigma_{\hat{a} \hat{b}}$ respectively with the following symmetry properties

$$
V_{\hat{\alpha} \hat{\beta}}=-V_{\hat{\beta} \hat{\alpha}}, \quad \varepsilon^{\hat{\alpha} \hat{\beta}} V_{\hat{\alpha} \hat{\beta}}=0, \quad F_{\hat{\alpha} \hat{\beta}}=F_{\hat{\beta} \hat{\alpha}} .
$$

The equivalent descriptions of $V_{\hat{a}}$ and $F_{\hat{a} \hat{b}}$ by $V_{\hat{\alpha} \hat{\beta}}$ and $F_{\hat{\alpha} \hat{\beta}}$ are explicitly related as follows:

$$
\begin{array}{ll}
V_{\hat{\alpha} \hat{\beta}}=V^{\hat{a}}\left(\Gamma_{\hat{a}}\right)_{\hat{\alpha} \hat{\beta}}, & V_{\hat{a}}=-\frac{1}{4}\left(\Gamma_{\hat{a}}\right)^{\hat{\alpha} \hat{\beta}} V_{\hat{\alpha} \hat{\beta}}, \\
F_{\hat{\alpha} \hat{\beta}}=\frac{1}{2} F^{\hat{a} \hat{b}}\left(\Sigma_{\hat{a} \hat{b}}\right)_{\hat{\alpha} \hat{\beta}}, & F_{\hat{a} \hat{b}}=\left(\Sigma_{\hat{a} \hat{b}}\right)^{\hat{\alpha} \hat{\beta}} F_{\hat{\alpha} \hat{\beta}} .
\end{array}
$$

This means that we may decompose an arbitrary tensor with two spinor indices, $T_{\hat{\alpha} \hat{\beta}}$, as follows

$$
T_{\hat{\alpha} \hat{\beta}}=\frac{1}{2}\left(\Sigma^{\hat{a} \hat{b}}\right)_{\hat{\alpha} \hat{\beta}}\left(\Sigma_{\hat{a} \hat{b}}\right)^{\hat{\gamma} \hat{\delta}} T_{\hat{\gamma} \hat{\delta}}-\frac{1}{4}\left(\left(\Gamma^{\hat{a}}\right)_{\hat{\alpha} \hat{\beta}}\left(\Gamma_{\hat{a}}\right)^{\hat{\gamma} \hat{\delta}}+\varepsilon_{\hat{\alpha} \hat{\beta}} \varepsilon^{\hat{\gamma} \hat{\delta}}\right) T_{\hat{\gamma} \hat{\delta}}
$$

These results may be checked using the identities

$$
\begin{aligned}
\varepsilon_{\hat{\alpha} \hat{\beta} \hat{\gamma} \hat{\delta}} & =\varepsilon_{\hat{\alpha} \hat{\beta}} \varepsilon_{\hat{\gamma} \hat{\delta}}+\varepsilon_{\hat{\alpha} \hat{\gamma}} \varepsilon_{\hat{\delta} \hat{\beta}}+\varepsilon_{\hat{\alpha} \hat{\delta}} \varepsilon_{\hat{\beta} \hat{\gamma}} \\
& =\frac{1}{2}\left(\Gamma^{\hat{a}}\right)_{\hat{\alpha} \hat{\beta}}\left(\Gamma_{\hat{a}}\right)_{\hat{\gamma} \hat{\delta}}+\frac{1}{2} \varepsilon_{\hat{\alpha} \hat{\beta}} \varepsilon_{\hat{\gamma} \hat{\delta}},
\end{aligned}
$$

where $\varepsilon_{\hat{\alpha} \hat{\beta} \hat{\gamma} \hat{\delta}}$ is the completely antisymmetric fourth-rank tensor.

The conjugation rules give

$$
\left(\varepsilon_{\hat{\alpha} \hat{\beta}}\right)^{*}=-\varepsilon^{\hat{\alpha} \hat{\beta}}, \quad\left(V_{\hat{\alpha} \hat{\beta}}\right)^{*}=V^{\hat{\alpha} \hat{\beta}}, \quad\left(F_{\hat{\alpha} \hat{\beta}}\right)^{*}=F^{\hat{\alpha} \hat{\beta}},
$$

provided $V^{\hat{a}}$ and $F^{\hat{a} \hat{b}}$ are real.

One can derive a number of identities involving the contraction of vector indices. These are listed below:

$$
\begin{aligned}
& \left(\Gamma^{\hat{a}}\right)_{\hat{\alpha} \hat{\beta}}\left(\Gamma_{\hat{a}}\right)_{\hat{\gamma} \hat{\delta}}=\varepsilon_{\hat{\alpha} \hat{\beta}} \varepsilon_{\hat{\gamma} \hat{\delta}}-2 \varepsilon_{\hat{\alpha} \hat{\gamma}} \varepsilon_{\hat{\beta} \hat{\delta}}+2 \varepsilon_{\hat{\alpha} \hat{\delta}} \varepsilon_{\hat{\beta} \hat{\gamma}}, \\
& \left(\Sigma_{\hat{a} \hat{b}}\right)_{\hat{\alpha} \hat{\beta}}\left(\Gamma^{\hat{b}}\right)_{\hat{\gamma} \hat{\delta}}=\frac{1}{2}\left(\left(\Gamma_{\hat{a}}\right)_{\hat{\alpha} \hat{\delta}} \varepsilon_{\hat{\beta} \hat{\gamma}}-\left(\Gamma_{\hat{a}}\right)_{\hat{\alpha} \hat{\gamma}} \varepsilon_{\hat{\beta} \hat{\delta}}+\left(\Gamma_{\hat{a}}\right)_{\hat{\beta} \hat{\delta}} \varepsilon_{\hat{\alpha} \hat{\gamma}}-\left(\Gamma_{\hat{a}}\right)_{\hat{\beta} \hat{\gamma}} \varepsilon_{\hat{\alpha} \hat{\delta}}\right) \text {, } \\
& \left(\Sigma^{\hat{a} \hat{b}}\right)_{\hat{\alpha} \hat{\beta}}\left(\Sigma_{\hat{a} \hat{b}}\right)_{\hat{\gamma} \hat{\delta}}=\varepsilon_{\hat{\alpha} \hat{\gamma}} \varepsilon_{\hat{\beta} \hat{\delta}}+\varepsilon_{\hat{\alpha} \hat{\delta}} \varepsilon_{\hat{\beta} \hat{\gamma}}, \\
& \varepsilon_{\hat{a} \hat{b} \hat{c} \hat{d} \hat{e}}\left(\Gamma^{\hat{c}}\right)_{\hat{\alpha} \hat{\beta}}\left(\Sigma^{\hat{d} \hat{e}}\right)_{\hat{\gamma} \hat{\delta}}=2 \varepsilon_{\hat{\alpha} \hat{\beta}}\left(\Sigma_{\hat{a} \hat{b}}\right)_{\hat{\gamma} \hat{\delta}}+2 \varepsilon_{\hat{\gamma} \hat{\alpha}}\left(\Sigma_{\hat{a} \hat{b}}\right)_{\hat{\beta} \hat{\delta}}+2 \varepsilon_{\hat{\delta} \hat{\alpha}}\left(\Sigma_{\hat{a} \hat{b}}\right)_{\hat{\beta} \hat{\gamma}} \\
& -2 \varepsilon_{\hat{\gamma} \hat{\beta}}\left(\Sigma_{\hat{a} \hat{b}}\right)_{\hat{\alpha} \hat{\delta}}-2 \varepsilon_{\hat{\delta} \hat{\beta}}\left(\Sigma_{\hat{a} \hat{b}}\right)_{\hat{\alpha} \hat{\gamma}},
\end{aligned}
$$

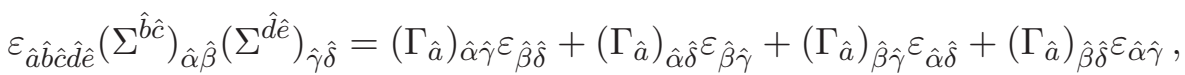

where the Levi-Civita tensor $\varepsilon_{\hat{a} \hat{b} \hat{c} \hat{d} \hat{e}}$ is defined to be completely antisymmetric with normalization

$$
\varepsilon_{01235}=-\varepsilon^{01235}=1 .
$$


The Levi-Civita tensor also satisfies the useful identity

$$
\varepsilon^{\hat{a}_{1} \cdots \hat{a}_{r} \hat{a}_{r+1} \cdots a_{5}} \varepsilon_{\hat{b}_{1} \cdots \hat{b}_{r} \hat{a}_{r+1} \cdots a_{5}}=-r !(5-r) ! \delta_{\hat{b}_{1}}^{\left[\hat{a}_{1}\right.} \cdots \delta_{\hat{b}_{r}}^{\left.\hat{a}_{r}\right]} .
$$

Some other useful relations are given by

$$
\begin{gathered}
\left(\Gamma^{[\hat{a}}\right)_{\hat{\alpha} \hat{\beta}}\left(\Gamma^{\hat{b}]}\right)_{\hat{\gamma} \hat{\delta}}=\varepsilon_{\hat{\alpha} \hat{\gamma}}\left(\Sigma^{\hat{a} \hat{b}}\right)_{\hat{\beta} \hat{\delta}}+\varepsilon_{\hat{\beta} \hat{\delta}}\left(\sum^{\hat{a} \hat{b}}\right)_{\hat{\alpha} \hat{\gamma}}-\varepsilon_{\hat{\alpha} \hat{\delta}}\left(\sum^{\hat{a} \hat{b}}\right)_{\hat{\beta} \hat{\gamma}}-\varepsilon_{\hat{\beta} \hat{\gamma}}\left(\sum^{\hat{a} \hat{b}}\right)_{\hat{\alpha} \hat{\delta}}, \\
\left(\Sigma^{\hat{c}[\hat{a}}\right)_{\hat{\alpha} \hat{\beta}}\left(\sum_{\hat{c}}^{\hat{b}]}\right)_{\hat{\gamma} \hat{\delta}}=-\frac{1}{4}\left(\varepsilon_{\hat{\alpha} \hat{\gamma}}\left(\Sigma^{\hat{a} \hat{b}}\right)_{\hat{\beta} \hat{\delta}}+\varepsilon_{\hat{\alpha} \hat{\delta}}\left(\Sigma^{\hat{a} \hat{b}}\right)_{\hat{\beta} \hat{\gamma}}+\varepsilon_{\hat{\beta} \hat{\gamma}}\left(\Sigma^{\hat{a} \hat{b}}\right)_{\hat{\alpha} \hat{\delta}}+\varepsilon_{\hat{\beta} \hat{\delta}}\left(\Sigma^{\hat{a} \hat{b}}\right)_{\hat{\alpha} \hat{\gamma}}\right),
\end{gathered}
$$

and

$$
\begin{aligned}
& \left(\Gamma^{\hat{a}} \Gamma^{\hat{b}}\right)_{\hat{\alpha}}^{\hat{\beta}}=\left(\Gamma^{\hat{a}}\right)_{\hat{\alpha}} \hat{\gamma}\left(\Gamma^{\hat{b}}\right)_{\hat{\gamma}}^{\hat{\beta}}=-\eta^{\hat{a} \hat{b}} \delta_{\hat{\alpha}}^{\hat{\beta}}-2\left(\Sigma^{\hat{a} \hat{b}}\right)_{\hat{\alpha}}^{\hat{\beta}}, \\
& \left.\left(\Gamma^{\hat{a}} \Gamma^{\hat{b}} \Gamma^{\hat{c}}\right)_{\hat{\alpha}}^{\hat{\beta}}=\left(-\eta^{\hat{a} \hat{b}} \eta^{\hat{c} \hat{d}}+\eta^{\hat{c} \hat{a}} \eta^{\hat{b} \hat{d}}-\eta^{\hat{b} \hat{c}} \eta^{\hat{a} \hat{d}}\right)\left(\Gamma_{\hat{d}}\right)_{\hat{\alpha}}^{\hat{\beta}}+\varepsilon^{\hat{a} b \hat{b} \hat{d} \hat{e}}\left(\Sigma_{\hat{d} \hat{e}}\right)\right)_{\hat{\alpha}}^{\hat{\beta}}, \\
& \left(\Gamma^{\hat{a}} \Gamma^{\hat{b}} \Gamma^{\hat{c}} \Gamma^{\hat{d}}\right)_{\hat{\alpha}}^{\hat{\beta}}=\left(\eta^{\hat{a} \hat{b}} \eta^{\hat{c} \hat{d}}-\eta^{\hat{a} \hat{c}} \eta^{\hat{b} \hat{d}}+\eta^{\hat{a} \hat{d}} \eta^{\hat{b} \hat{c}}\right) \delta_{\hat{\alpha}}^{\hat{\beta}}-\varepsilon^{\hat{a} \hat{b} \hat{c} \hat{d} \hat{e}}\left(\Gamma_{\hat{e}}\right)_{\hat{\alpha}} \hat{\beta} \\
& +2 \eta^{\hat{a} \hat{b}}\left(\Sigma^{\hat{c} \hat{d}}\right)_{\hat{\alpha}} \hat{\beta}-2 \eta^{\hat{a} \hat{c}}\left(\Sigma^{\hat{b} \hat{d}}\right)_{\hat{\alpha}} \hat{\beta}+2 \eta^{\hat{b} \hat{c}}\left(\Sigma^{\hat{a} \hat{d}}\right)_{\hat{\alpha}}^{\hat{\beta}} \\
& +2 \eta^{\hat{d} \hat{c}}\left(\Sigma^{\hat{a} \hat{b}}\right)_{\hat{\alpha}}{ }_{\hat{\beta}}-2 \eta^{\hat{d} \hat{b}}\left(\Sigma^{\hat{a} \hat{c}}\right)_{\hat{\alpha}}{ }_{\hat{\beta}} \hat{\beta}+2 \eta^{\hat{d} \hat{a}}\left(\Sigma^{\hat{b} \hat{c}}\right)_{\hat{\alpha}} \hat{\beta}^{\hat{\beta}}, \\
& \left(\Gamma^{\hat{a}} \Gamma^{\hat{b}} \Gamma^{\hat{c}} \Gamma^{\hat{d}} \Gamma^{\hat{e}}\right)_{\hat{\alpha}}^{\hat{\beta}}=\varepsilon^{\hat{a} b \hat{b} \hat{c} \hat{e} \hat{e}} \delta_{\hat{\alpha}}^{\hat{\beta}}+\left(\Gamma^{\hat{a}}\right)_{\hat{\alpha}}^{\hat{\beta}}\left(\eta^{\hat{b} \hat{c}} \eta^{\hat{d} \hat{e}}-\eta^{\hat{b} \hat{d}} \eta^{\hat{c} \hat{e}}+\eta^{\hat{c} \hat{d}} \eta^{\hat{b} \hat{e}}\right) \\
& +\left(\Gamma^{\hat{b}}\right)_{\hat{\alpha}}^{\hat{\beta}}\left(-\eta^{\hat{c} \hat{d}} \eta^{\hat{e} \hat{a}}+\eta^{\hat{c} \hat{e}} \eta^{\hat{d} \hat{a}}-\eta^{\hat{d} \hat{e}} \eta^{\hat{c} \hat{a}}\right) \\
& +\left(\Gamma^{\hat{c}}\right)_{\hat{\alpha}}^{\hat{\beta}}\left(\eta^{\hat{d} \hat{e}} \eta^{\hat{a} \hat{b}}-\eta^{\hat{d} \hat{a}} \eta^{\hat{e} \hat{b}}+\eta^{\hat{e} \hat{a}} \eta^{\hat{d} \hat{b}}\right) \\
& +\left(\Gamma^{\hat{d}}\right)_{\hat{\alpha}}^{\hat{\beta}}\left(-\eta^{\hat{e} \hat{a}} \eta^{\hat{b} \hat{c}}+\eta^{\hat{e} \hat{b}} \eta^{\hat{a} \hat{c}}-\eta^{\hat{a} \hat{b}} \eta^{\hat{e} \hat{c}}\right) \\
& +\left(\Gamma^{\hat{e}}\right)_{\hat{\alpha}}^{\hat{\beta}}\left(\eta^{\hat{a} \hat{b}} \eta^{\hat{c} \hat{d}}-\eta^{\hat{c} \hat{a}} \eta^{\hat{b} \hat{d}}+\eta^{\hat{b} \hat{c}} \eta^{\hat{a} \hat{d}}\right)+2 \varepsilon^{\hat{a} \hat{b} \hat{c} \hat{d} \hat{m}}\left(\Sigma_{\hat{m}}{ }^{\hat{e}}\right)_{\hat{\alpha}}^{\hat{\beta}} \\
& +\left(\Sigma_{\hat{m} \hat{n}}\right)_{\hat{\alpha}}^{\hat{\beta}}\left(-\eta^{\hat{a} \hat{b}} \varepsilon^{\hat{c} \hat{d} \hat{e} \hat{m} \hat{n}}+\eta^{\hat{c} \hat{a}} \varepsilon^{\hat{b} \hat{d} \hat{e} \hat{m} \hat{n}}-\eta^{\hat{b} \hat{c}} \varepsilon^{\hat{a} \boldsymbol{d} \hat{e} \hat{m} \hat{n}}\right. \\
& \left.-\eta^{\hat{d} \hat{a}} \varepsilon^{\hat{b} c \hat{e} \hat{m} \hat{n}}+\eta^{\hat{d} \hat{b}} \varepsilon^{\hat{a} \hat{c} \hat{c} \hat{m} \hat{n}}-\eta^{\hat{d} \hat{c}} \varepsilon^{\hat{a} \hat{b} \hat{e} \hat{m} \hat{n}}\right) .
\end{aligned}
$$

\section{B The conformal Killing supervector fields of $\mathbb{R}^{5 \mid 8}$}

The 5D superconformal algebra $\mathrm{F}^{2}(4)$ [27] can be identified with the algebra of conformal Killing supervector fields of $5 \mathrm{D} \mathcal{N}=1$ Minkowski superspace [33]. In this appendix we spell out this construction.

Simple Minkowski superspace in five dimensions, $\mathbb{R}^{5 \mid 8}$, is parametrized by coordinates $z^{\hat{A}}=\left(x^{\hat{a}}, \theta_{i}^{\hat{\alpha}}\right)$. The flat covariant derivatives $D_{\hat{A}}=\left(\partial_{\hat{a}}, D_{\hat{\alpha}}^{i}\right)$

$$
\partial_{\hat{a}}:=\frac{\partial}{\partial x^{\hat{a}}}, \quad D_{\hat{\alpha}}^{i}:=\frac{\partial}{\partial \theta_{i}^{\hat{\alpha}}}-\mathrm{i}\left(\Gamma^{\hat{b}}\right)_{\hat{\alpha} \hat{\beta}} \theta^{\hat{\beta} i} \partial_{\hat{b}},
$$

satisfy the algebra:

$$
\left\{D_{\hat{\alpha}}^{i}, D_{\hat{\beta}}^{j}\right\}=-2 \mathrm{i}\left(\Gamma^{\hat{a}}\right)_{\hat{\alpha} \hat{\beta}} \partial_{\hat{a}}, \quad\left[\partial_{\hat{a}}, D_{\hat{\beta}}^{j}\right]=0, \quad\left[\partial_{\hat{a}}, \partial_{\hat{b}}\right]=0 .
$$

The spinor covariant derivatives satisfy the reality condition $\left(D_{\hat{\alpha}}^{i} F\right)^{*}=-(-1)^{\varepsilon(F)} D_{i}^{\hat{\alpha}} \bar{F}$ with $F$ an arbitrary superfield of Grassmann parity $\varepsilon(F)$. 
According to [33], the conformal Killing supervector fields

$$
\xi=\bar{\xi}=\xi^{\hat{a}}(z) \partial_{\hat{a}}+\xi_{i}^{\hat{\alpha}}(z) D_{\hat{\alpha}}^{i}
$$

are defined to satisfy

$$
\left[\xi, D_{\hat{\alpha}}^{i}\right]=-\left(D_{\hat{\alpha}}^{i} \xi_{j}^{\hat{\beta}}\right) D_{\hat{\beta}}^{j},
$$

which implies the fundamental equation

$$
D_{\hat{\alpha}}^{i} \xi_{\hat{a}}=2 \mathrm{i}\left(\Gamma_{\hat{a}}\right)_{\hat{\alpha}}^{\hat{\beta}} \xi_{\hat{\beta}}^{i}
$$

From eq. (B.5) one finds

$$
\varepsilon^{i j}\left(\Gamma_{\hat{a}}\right)_{\hat{\alpha} \hat{\beta}} \partial^{\hat{a}} \xi^{\hat{b}}=\left(\Gamma^{\hat{b}}\right)_{\hat{\alpha} \hat{\gamma}} D_{\hat{\beta}}^{j} \xi^{\hat{\gamma} i}+\left(\Gamma^{\hat{b}}\right)_{\hat{\beta} \hat{\gamma}} D_{\hat{\alpha}}^{i} \xi^{\hat{\gamma} j}
$$

which gives us the usual equation for a conformal Killing vector field

$$
\partial_{(\hat{a}} \xi_{\hat{b})}=\frac{1}{5} \eta_{\hat{a} \hat{b}} \partial^{\hat{c}} \xi_{\hat{c}}
$$

The conformal Killing vector acts on the spinor covariant derivatives as

$$
\left[\xi, D_{\hat{\alpha}}^{i}\right]=-\omega_{\hat{\alpha}}^{\hat{\beta}} D_{\hat{\beta}}^{i}+\Lambda^{i j} D_{\hat{\alpha} j}-\frac{1}{2} \sigma D_{\hat{\alpha}}^{i},
$$

where the parameters $\omega_{\hat{\alpha} \hat{\beta}}, \sigma$ and $\Lambda^{i j}$ are given by the following expressions:

$$
\begin{aligned}
\omega_{\hat{\alpha} \hat{\beta}} & :=\frac{1}{2} D_{(\hat{\alpha}}^{k} \xi_{\hat{\beta}) k}=\frac{1}{2}\left(\Sigma^{\hat{a} \hat{b}}\right)_{\hat{\alpha} \hat{\beta}} \partial_{\hat{a}} \xi_{\hat{b}}, \\
\sigma & :=\frac{1}{4} D_{k}^{\hat{\alpha}} \xi_{\hat{\alpha}}^{k}=\frac{1}{5} \partial^{\hat{a}} \xi_{\hat{a}}, \\
\Lambda^{i j} & :=\frac{1}{4} D_{\hat{\gamma}}^{(i} \xi^{\hat{\gamma} j)} .
\end{aligned}
$$

As a consequence of eq. (B.7) we find the parameters satisfy the identities

$$
\begin{aligned}
\partial_{\hat{a}} \omega_{\hat{b} \hat{c}} & =-2 \eta_{\hat{a}[\hat{b}} \partial_{\hat{c}]} \sigma, \\
\partial_{\hat{a}} \partial_{\hat{b}} \xi_{\hat{c}} & =-\eta_{\hat{a} \hat{b}} \partial_{\hat{c}} \sigma+2 \eta_{\hat{c}(\hat{a}} \partial_{\hat{b})} \sigma .
\end{aligned}
$$

Furthermore, as a consequence of eq. (B.5) we also find

$$
\begin{aligned}
& D_{\hat{\gamma}}^{k} \omega_{\hat{\alpha} \hat{\beta}}=-2 \varepsilon_{\hat{\gamma}(\hat{\alpha}} D_{\hat{\beta})}^{k} \sigma, \\
& D_{\hat{\alpha}}^{i} \Lambda^{j k}=3 \varepsilon^{i(j} D_{\hat{\alpha}}^{k)} \sigma,
\end{aligned}
$$

where $\sigma$ obeys

$$
D_{\hat{\alpha}}^{i} D_{\hat{\beta}}^{j} \sigma=-\mathrm{i} \varepsilon^{i j}\left(\Gamma^{\hat{a}}\right)_{\hat{\alpha} \hat{\beta}} \partial_{\hat{a}} \sigma
$$

and

$$
\partial_{\hat{a}} D_{\hat{\beta}}^{j} \sigma=0 .
$$

The above results tell us that we can parametrize superconformal Killing vectors as follows

$$
\xi \equiv \xi\left(\Lambda(P)^{\hat{a}}, \Lambda(Q)_{i}^{\hat{\alpha}}, \Lambda(M)_{\hat{a} \hat{b}}, \Lambda(\mathbb{D}), \Lambda(K)^{\hat{a}}, \Lambda(S)^{\hat{\alpha} i}\right)
$$


where we have defined

$$
\begin{array}{rlrl}
\Lambda(P)^{\hat{a}} & :=-\left.\xi^{\hat{a}}\right|_{x=\theta=0}, & \Lambda(Q)_{i}^{\hat{\alpha}} & =-\left.\xi_{i}^{\hat{\alpha}}\right|_{x=\theta=0}, \\
\Lambda(M)_{\hat{a} \hat{b}} & :=\left.\omega_{\hat{a} \hat{b}}\right|_{x=\theta=0}, & \Lambda(\mathbb{D}) & :=\left.\sigma\right|_{x=\theta=0}, \\
\Lambda(K)_{\hat{a}} & :=-\left.\frac{1}{2} \partial_{\hat{a}} \sigma\right|_{x=\theta=0}, & \Lambda(S)^{\hat{\alpha} i}:=-\left.\frac{1}{2} D^{\hat{\alpha} i} \sigma\right|_{x=\theta=0} .
\end{array}
$$

The commutator of two superconformal Killing vectors,

$$
\xi=\xi\left(\Lambda(P)^{\hat{a}}, \Lambda(Q)_{i}^{\hat{\alpha}}, \Lambda(M)_{\hat{a} \hat{b}}, \Lambda(\mathbb{D}), \Lambda(K)^{\hat{a}}, \Lambda(S)^{\hat{\alpha} i}\right)
$$

and

$$
\tilde{\xi}=\xi\left(\tilde{\Lambda}(P)^{\hat{a}}, \tilde{\Lambda}(Q)_{i}^{\hat{\alpha}}, \tilde{\Lambda}(M)_{\hat{a} \hat{b}}, \tilde{\Lambda}(\mathbb{D}), \tilde{\Lambda}(K)_{\hat{a}}, \tilde{\Lambda}(S)^{\hat{\alpha} i}\right),
$$

is another superconformal Killing vector given by

$$
\begin{aligned}
{[\xi, \tilde{\xi}]=} & \left(\xi^{\hat{a}} \partial_{\hat{a}} \tilde{\xi}^{\hat{b}}-\tilde{\xi}^{\hat{a}} \partial_{\hat{a}} \xi^{\hat{b}}+\xi_{i}^{\hat{\alpha}} D_{\hat{\alpha}}^{i} \tilde{\xi}^{\hat{b}}-\tilde{\xi}_{i}^{\hat{\alpha}} D_{\hat{\alpha}}^{i} \xi^{\hat{b}}+2 \mathrm{i} \xi_{k}^{\hat{\alpha}} \tilde{\xi}^{\hat{\beta} k}\left(\Gamma^{\hat{b}}\right)_{\hat{\alpha} \hat{\beta}}\right) \partial_{\hat{b}} \\
& +\left(\xi^{\hat{a}} \partial_{\hat{a}} \tilde{\xi}_{j}^{\hat{\beta}}-\tilde{\xi}^{\hat{a}} \partial_{\hat{a}} \xi_{j}^{\hat{\beta}}+\xi_{i}^{\hat{\alpha}} D_{\hat{\alpha}}^{i} \tilde{\xi}_{j}^{\hat{\beta}}-\tilde{\xi}_{i}^{\hat{\alpha}} D_{\hat{\alpha}}^{i} \xi_{j}^{\hat{\beta}}\right) D_{\hat{\beta}}^{j} \\
\equiv & \xi\left(\hat{\Lambda}^{\hat{a}}(P), \hat{\Lambda}_{i}^{\hat{\alpha}}(Q), \hat{\Lambda}(M)_{\hat{a} \hat{b}}, \hat{\Lambda}(\mathbb{D}), \hat{\Lambda}(K)^{\hat{a}}, \hat{\Lambda}(S)^{\hat{\alpha} i}\right),
\end{aligned}
$$

where

$$
\begin{aligned}
& \hat{\Lambda}^{\hat{a}}(P):=\Lambda(P)^{\hat{b}} \tilde{\Lambda}_{\hat{b}}^{\hat{a}}+\Lambda(P)^{\hat{a}} \tilde{\Lambda}(\mathbb{D})-2 \operatorname{i} \Lambda(Q)_{k}^{\hat{\alpha}} \tilde{\Lambda}(Q)^{\hat{\beta} k}\left(\Gamma^{\hat{a}}\right)_{\hat{\alpha} \hat{\beta}} \\
& -\tilde{\Lambda}(P)^{\hat{b}} \Lambda_{\hat{b}}^{\hat{a}}-\tilde{\Lambda}(P)^{\hat{a}} \Lambda(\mathbb{D}), \\
& \hat{\Lambda}_{i}^{\hat{\alpha}}(Q):=-\mathrm{i}\left(\Gamma_{\hat{a}}\right)^{\hat{\alpha} \hat{\beta}} \Lambda(P)^{\hat{a}} \tilde{\Lambda}(S)_{\hat{\beta} i}+\Lambda(Q)_{i}^{\hat{\beta}} \tilde{\Lambda}(M)_{\hat{\beta}}^{\hat{\alpha}}+\frac{1}{2} \Lambda(Q)_{i}^{\hat{\alpha}} \tilde{\Lambda}(\mathbb{D})+\Lambda(Q)_{j}^{\hat{\alpha}} \tilde{\Lambda}(J)^{j}{ }_{i} \\
& +\mathrm{i}\left(\Gamma_{\hat{a}}\right)^{\hat{\alpha} \hat{\beta}} \tilde{\Lambda}(P)^{\hat{a}} \Lambda(S)_{\hat{\beta} i}-\tilde{\Lambda}(Q)_{i}^{\hat{\beta}} \Lambda(M)_{\hat{\beta}}^{\hat{\alpha}}-\frac{1}{2} \tilde{\Lambda}(Q)_{i}^{\hat{\alpha}} \Lambda(\mathbb{D})-\tilde{\Lambda}(Q)_{j}^{\hat{\alpha}} \Lambda(J)_{i}^{j}, \\
& \hat{\Lambda}(M)_{\hat{a} \hat{b}}:=2 \Lambda(M)^{\hat{c}}{ }_{[\hat{a}} \tilde{\Lambda}(M)_{\hat{b}] \hat{c}}-4 \Lambda(P)_{[\hat{a}} \tilde{\Lambda}(K)_{\hat{b}]}+4 \tilde{\Lambda}(P)_{[\hat{a}} \Lambda(K)_{\hat{b}]}, \\
& \hat{\Lambda}(\mathbb{D}):=2 \Lambda(P)^{\hat{a}} \tilde{\Lambda}(K)_{\hat{a}}-2 \tilde{\Lambda}(P)^{\hat{a}} \Lambda(K)_{\hat{a}}+2 \Lambda(S)^{\hat{\alpha} i} \tilde{\Lambda}(Q)_{\hat{\alpha} i}-2 \tilde{\Lambda}(S)^{\hat{\alpha} i} \Lambda(Q)_{\hat{\alpha} i}, \\
& \hat{\Lambda}(K)^{\hat{a}}:=\Lambda(M)^{\hat{a} \hat{b}} \tilde{\Lambda}(K)_{\hat{b}}+\Lambda(\mathbb{D}) \tilde{\Lambda}(K)^{\hat{a}}-2 \mathrm{i} \Lambda(S)_{k}^{\hat{\alpha}} \tilde{\Lambda}(S)^{\hat{\beta} k}\left(\Gamma^{\hat{a}}\right)_{\hat{\alpha} \hat{\beta}} \\
& -\tilde{\Lambda}(M)^{\hat{a} \hat{b}} \Lambda(K)_{\hat{b}}-\tilde{\Lambda}(\mathbb{D}) \Lambda(K)^{\hat{a}}, \\
& \hat{\Lambda}(S)^{\hat{\alpha} i}:=\mathrm{i}\left(\Gamma_{\hat{a}}\right)^{\hat{\alpha} \hat{\beta}} \Lambda(K)^{\hat{a}} \tilde{\Lambda}(Q)_{\hat{\beta}}^{i}+\Lambda(S)^{\hat{\beta} i} \tilde{\Lambda}(M)_{\hat{\beta}}^{\hat{\alpha}}-\frac{1}{2} \Lambda(S)^{\hat{\alpha} i} \tilde{\Lambda}(\mathbb{D})+\Lambda(S)_{j}^{\hat{\alpha}} \tilde{\Lambda}(J)^{j i} \\
& -\mathrm{i}\left(\Gamma_{\hat{a}}\right)^{\hat{\alpha} \hat{\beta}} \tilde{\Lambda}(K)^{\hat{a}} \Lambda(Q)_{\hat{\beta}}^{i}-\tilde{\Lambda}(S)^{\hat{\beta} i} \Lambda(M)_{\hat{\beta}}^{\hat{\alpha}}+\frac{1}{2} \tilde{\Lambda}(S)^{\hat{\alpha} i} \Lambda(\mathbb{D})-\tilde{\Lambda}(S)_{j}^{\hat{\alpha}} \Lambda(J)^{j i} .
\end{aligned}
$$

Associating with the superconformal Killing vector $\xi$ the transformation

$$
\delta_{\xi}=\Lambda(P)^{\hat{a}} P_{\hat{a}}+\Lambda(Q)_{i}^{\hat{\alpha}} Q_{\hat{\alpha}}^{i}+\frac{1}{2} \Lambda(M)^{\hat{a} \hat{b}} M_{\hat{a} \hat{b}}+\Lambda(\mathbb{D}) \mathbb{D}+\Lambda(K)^{\hat{a}} K_{\hat{a}}+\Lambda(S)^{\hat{\alpha} i} S_{\hat{\alpha} i}
$$

and comparing to the above gives us the superconformal algebra (2.1). 


\section{Modified superspace algebra}

In section 4, we introduced a modified definition of the composite vector connections. It is actually possible to introduce this redefinition directly within the context of superspace. The modified superspace vector derivative is

$$
\begin{aligned}
\hat{\nabla}_{\hat{a}}= & \nabla_{\hat{a}}-\frac{1}{4} \tilde{W}_{\hat{a} \hat{b} \hat{c}} M^{\hat{b} \hat{c}}+\frac{1}{8} X^{\hat{\beta} i}\left(\Gamma_{\hat{a}}\right)_{\hat{\beta}}^{\hat{\alpha}} S_{\hat{\alpha} i}+\frac{1}{64}\left(Y+3 W^{\hat{b} \hat{c}} W_{\hat{b} \hat{c}}\right) K_{\hat{a}} \\
& -\frac{1}{4}\left(\nabla^{\hat{c}} \tilde{W}_{\hat{c} \hat{b}}{ }^{\hat{b}}\right) K_{\hat{b}}-\frac{1}{4} W_{\hat{a} \hat{d}} W^{\hat{b} \hat{d}} K_{\hat{b}} .
\end{aligned}
$$

The new vector derivative possesses a deformed $S$-supersymmetry transformation, but it retains the original $K$-transformation,

$$
\begin{aligned}
{\left[S_{\hat{\beta} i}, \hat{\nabla}_{\hat{a}}\right] } & =\mathrm{i}\left(\Gamma_{\hat{a}}\right)_{\hat{\beta}}{ }^{\hat{\alpha}} \nabla_{\hat{\alpha} i}-\frac{1}{2} W_{\hat{a}}^{\hat{b}}\left(\Gamma_{\hat{b}}\right)_{\hat{\beta}}{ }^{\hat{\alpha}} S_{\hat{\alpha} i}+\frac{\mathrm{i}}{8}\left(\Gamma_{\hat{a}} \Gamma^{\hat{b}}\right)_{\hat{\beta}}^{\hat{\gamma}} X_{\hat{\gamma} i} K_{\hat{b}}-\frac{\mathrm{i}}{4} W_{\hat{a} \hat{b} \hat{\beta} i} K^{\hat{b}}, \\
{\left[K_{\hat{b}}, \hat{\nabla}_{\hat{a}}\right] } & =2 \eta_{\hat{a} \hat{b}} \mathbb{D}+2 M_{\hat{a} \hat{b}} .
\end{aligned}
$$

The spinor derivative remains unchanged, $\hat{\nabla}_{\hat{\alpha}}^{i}=\nabla_{\hat{\alpha}}^{i}$.

The new curvature tensors, given in their general form as

$$
\begin{aligned}
{\left[\hat{\nabla}_{\hat{A}}, \hat{\nabla}_{\hat{B}}\right]=} & -\hat{\mathscr{T}}_{\hat{A} \hat{B}} \hat{C} \hat{\nabla}_{\hat{C}}-\frac{1}{2} \hat{\mathscr{R}}(M)_{\hat{A} \hat{B}}{ }^{\hat{c} \hat{d}} M_{\hat{c} \hat{d}}-\hat{\mathscr{R}}(D)_{\hat{A} \hat{B}} \mathbb{D} \\
& -\hat{\mathscr{R}}(J)_{\hat{A} \hat{B}}{ }^{i j} J_{i j}-\hat{\mathscr{R}}(S)_{\hat{A} \hat{B}}{ }^{\hat{\alpha} i} S_{\hat{\alpha} i}-\hat{\mathscr{R}}(K)_{\hat{A} \hat{B}}{ }^{\hat{c}} K_{\hat{c}}
\end{aligned}
$$

can be found by direct computation. For the algebra of two spinor derivatives, we find

$$
\begin{aligned}
\hat{\mathscr{T}}_{\hat{\alpha} \hat{\beta}}^{i j} \hat{c}= & 2 \mathrm{i} \varepsilon^{i j}\left(\Gamma^{\hat{c}}\right)_{\hat{\alpha} \hat{\beta}}, \\
\hat{\mathscr{T}}_{\hat{\alpha} \hat{\beta}}^{i j \hat{\gamma}}= & 0, \\
\hat{\mathscr{R}}(M)_{\hat{\alpha} \hat{\beta}}^{i j \hat{c} \hat{d}}= & 2 \mathrm{i} \varepsilon^{i j} \varepsilon_{\hat{\alpha} \hat{\beta}} W^{\hat{c} \hat{d}}+\mathrm{i} \varepsilon^{i j}\left(\Gamma_{\hat{b}}\right)_{\hat{\alpha} \hat{\beta}} \tilde{W}^{\hat{b} \hat{c} \hat{d}}, \\
\hat{\mathscr{R}}(\mathbb{D})_{\hat{\alpha} \hat{\beta}}^{i j}= & 0, \\
\hat{\mathscr{R}}(J)_{\hat{\alpha} \hat{\beta}}^{i j k l}= & 0 \\
\hat{\mathscr{R}}(S)_{\hat{\alpha} \hat{\beta}}^{i j \hat{\gamma} k}= & \frac{3 \mathrm{i}}{4} \varepsilon^{i j} \varepsilon_{\hat{\alpha} \hat{\beta}} X^{\hat{\gamma} k}+\mathrm{i} \varepsilon^{i j} \delta_{[\hat{\alpha}}^{\hat{\gamma}} X_{\hat{\beta}]}^{k}, \\
\hat{\mathscr{R}}(K)_{\hat{\alpha} \hat{\beta}}^{i j \hat{c}}= & -\frac{\mathrm{i}}{2} \varepsilon^{i j} \varepsilon_{\hat{\alpha} \hat{\beta}} \hat{\nabla}^{\hat{b}} W_{\hat{b}}^{\hat{c}}+\frac{\mathrm{i}}{2} \varepsilon^{i j}\left(\Gamma^{\hat{a}}\right)_{\hat{\alpha} \hat{\beta}} \hat{\nabla}^{\hat{d}} \tilde{W}_{\hat{d} \hat{a}}^{\hat{c}}-\frac{\mathrm{i}}{32} \varepsilon^{i j}\left(\Gamma^{\hat{c}}\right)_{\hat{\alpha} \hat{\beta}} Y \\
& +\frac{\mathrm{i}}{4} \varepsilon^{i j} \varepsilon_{\hat{\alpha} \hat{\beta}} \tilde{W}_{\hat{c}} \hat{\hat{d} \hat{e}} W_{\hat{d} \hat{e}}+\frac{\mathrm{i}}{2} \varepsilon^{i j}\left(\Gamma^{\hat{a}}\right)_{\hat{\alpha} \hat{\beta}}\left(W_{\hat{a} \hat{d}} W^{\hat{c} \hat{d}}-\frac{3}{16} W^{\hat{b} \hat{d}} W_{\hat{b} \hat{d}} \delta_{\hat{a}}^{\hat{c}}\right) .
\end{aligned}
$$

The spinor-vector commutators lead to

$$
\begin{aligned}
\hat{\mathscr{T}}_{\hat{b} \hat{\alpha}}^{i} \hat{c} & =0, \\
\hat{\mathscr{T}}_{\hat{b} \hat{\alpha} k}^{i \hat{\gamma}} & =\frac{1}{4} \delta_{k}^{i}\left(3\left(\Gamma_{\hat{b}}\right)_{\hat{\alpha}}^{\hat{\beta}} W_{\hat{\beta}}^{\hat{\gamma}}-W_{\hat{\alpha}}^{\hat{\beta}}\left(\Gamma_{\hat{b}}\right)_{\hat{\beta}}^{\hat{\gamma}}\right), \\
\hat{\mathscr{R}}(D)_{\hat{b} \hat{\alpha}}^{i} & =-\frac{1}{4}\left(\Gamma_{\hat{b}}\right)_{\hat{\alpha}}{ }^{\hat{\gamma}} X_{\hat{\gamma}}^{i},
\end{aligned}
$$




$$
\begin{aligned}
& \hat{\mathscr{R}}(J)_{\hat{b} \hat{\alpha}}^{i j k}=-\frac{3}{4}\left(\Gamma_{\hat{b}}\right)_{\hat{\alpha}}^{\hat{\gamma}} \varepsilon^{i(j} X_{\hat{\gamma}}^{k)}, \\
& \hat{\mathscr{R}}(M)_{\hat{b} \hat{\alpha}}^{i \hat{c} \hat{d}}=-\left(\Gamma_{\hat{b}}\right)_{\hat{\alpha}}^{\hat{\gamma}} W_{\hat{\gamma}}^{\hat{c} \hat{d} i}-\frac{1}{4} \varepsilon_{\hat{b}}^{\hat{c} \hat{d} \hat{e} \hat{f}} W_{\hat{e} \hat{f} \hat{\alpha}}^{i}+\frac{1}{2} \delta_{\hat{b}}^{[\hat{c}}\left(\Gamma^{\hat{d}]}\right)_{\hat{\alpha}}^{\hat{\gamma}} X_{\hat{\gamma}}^{i}, \\
& \hat{\mathscr{R}}(S)_{\hat{b} \hat{\alpha}}^{i \hat{\gamma} j}=\frac{1}{16} X_{\hat{c} \hat{d}}^{i j}\left(\Sigma^{\hat{c} \hat{d}} \Gamma_{\hat{b}}-2 \Gamma_{\hat{b}} \Sigma^{\hat{c} \hat{d}}\right)_{\hat{\alpha}}^{\hat{\gamma}} \\
& -\frac{3 \mathrm{i}}{8} \varepsilon^{i j} \hat{\nabla}_{[\hat{b}} W_{\hat{c} \hat{d}]}\left(\Sigma^{\hat{c} \hat{d}}\right)_{\hat{\alpha}} \hat{\gamma}-\frac{\mathrm{i}}{8} \varepsilon^{i j} \hat{\nabla}_{\hat{d}} W^{\hat{d} \hat{c}}\left(\Sigma_{\hat{c} \hat{b}}\right)_{\hat{\alpha}} \hat{\gamma} \\
& +\frac{3 \mathrm{i}}{16} \varepsilon^{i j} \hat{\nabla}^{\hat{d}} W_{\hat{d} \hat{b}} \delta_{\hat{\alpha}}^{\hat{\gamma}}-\frac{\mathrm{i}}{8} \varepsilon^{i j} \hat{\nabla}^{\hat{c}} \tilde{W}_{\hat{c} \hat{b}} \hat{d}_{\left(\Gamma_{\hat{d}}\right) \hat{\alpha}}^{\hat{\gamma}} \\
& +\frac{\mathrm{i}}{16} \varepsilon^{i j} \tilde{W}^{\hat{c} \hat{d} \hat{e}} W_{\hat{d} \hat{e}}\left(\Sigma_{\hat{c} \hat{b}}\right) \hat{\alpha}^{\hat{\gamma}}-\frac{3 \mathrm{i}}{32} \varepsilon^{i j} \tilde{W}_{\hat{b} \hat{d} \hat{e}} W^{\hat{d} \hat{e}} \delta_{\hat{\alpha}}^{\hat{\gamma}} \\
& +\frac{\mathrm{i}}{4} \varepsilon^{i j} W_{\hat{b} \hat{d}} W^{\hat{c} \hat{d}}\left(\Gamma_{\hat{c}}\right)_{\hat{\alpha}}^{\hat{\gamma}}-\frac{3 \mathrm{i}}{64} \varepsilon^{i j} W^{\hat{c} \hat{d}} W_{\hat{c} \hat{d}}\left(\Gamma_{\hat{b}}\right)_{\hat{\alpha}}^{\hat{\gamma}}, \\
& \hat{\mathscr{R}}(K)_{\hat{b} \hat{\alpha}}^{i \hat{c}}=\frac{1}{6}\left(\Gamma^{\hat{c}}\right)_{\hat{\alpha}}^{\hat{\beta}} \hat{\nabla}^{\hat{d}} W_{\hat{d} \hat{b} \hat{\beta}}^{i}+\frac{1}{12}\left(\Gamma_{\hat{b}}\right)_{\hat{\alpha}}^{\hat{\beta}} \hat{\nabla}^{\hat{d}} W_{\hat{d} \hat{\beta}}^{\hat{c} i}+\frac{1}{6} \hat{\nabla}_{\hat{\alpha}}^{\hat{\beta}} W_{\hat{b} \hat{\beta}}^{\hat{c} i}-\frac{1}{24} \varepsilon_{\hat{b}}^{\hat{c} \hat{d} \hat{e} \hat{f}} \hat{\nabla}_{\hat{d}} W_{\hat{e} \hat{f} \hat{\alpha}}^{i} \\
& +\frac{1}{8}\left(\Gamma^{\hat{c}}\right)_{\hat{\alpha}}^{\hat{\beta}} \hat{\nabla}_{\hat{b}} X_{\hat{\beta}}^{i}+\frac{1}{64} W^{\hat{d} \hat{e}}\left(3 \Gamma_{\hat{b}} \Sigma_{\hat{d} \hat{e}} \Gamma^{\hat{c}}-\Sigma_{\hat{d} \hat{e}} \Gamma_{\hat{b}} \Gamma^{\hat{c}}\right)_{\hat{\alpha}}^{\hat{\beta}} X_{\hat{\beta}}^{i} \\
& -\frac{1}{48} \tilde{W}_{\hat{b} \hat{d} \hat{e}}\left(\Gamma^{\hat{c}}\right)_{\hat{\alpha}}^{\hat{\beta}} W_{\hat{\beta}}^{\hat{d} \hat{e} i}+\frac{1}{8} \delta_{\hat{b}}^{\hat{c}} W^{\hat{d} \hat{e}} W_{\hat{d} \hat{e} \hat{\alpha}}^{i} \\
& +\frac{1}{12}\left(\Sigma_{\hat{b}}^{\hat{c}}\right)_{\hat{\alpha}}^{\hat{\beta}} W_{\hat{d} \hat{e} \hat{\beta}}^{i} W^{\hat{d} \hat{e}}-\frac{1}{12} W^{\hat{d} \hat{e}}\left(\Sigma_{\hat{d} \hat{e}}\right)_{\hat{\alpha}}^{\hat{\beta}} W_{\hat{b}}^{\hat{c}}{ }_{\hat{\beta}}^{i} \\
& +\frac{13}{48} W_{\hat{b} \hat{d}} W_{\hat{\alpha}}^{\hat{d} \hat{c} i}+\frac{11}{48} W_{\hat{b} \hat{d} \hat{\alpha}}^{i} W^{\hat{d} \hat{c}}-\frac{13}{96}\left(\Gamma_{\hat{b}}\right)_{\hat{\alpha}}^{\hat{\beta}} W_{\hat{d} \hat{e} \hat{\beta}}^{i} \tilde{W}^{\hat{d} \hat{e} \hat{c}} .
\end{aligned}
$$

The vector-vector commutator is given by

$$
\begin{aligned}
& \hat{\mathscr{T}}_{\hat{a} \hat{b}} \hat{c}=0, \\
& \hat{\mathscr{T}}_{\hat{a} \hat{b} i}^{\hat{\alpha}}=-\frac{i}{2} W_{\hat{a} \hat{b} i}^{\hat{\alpha}}, \\
& \hat{\mathscr{R}}(D)_{\hat{a} \hat{b}}=0 \text {, } \\
& \hat{\mathscr{R}}(J)_{\hat{a} \hat{b}}{ }^{i j}=-\frac{3 \mathrm{i}}{4} X_{\hat{a} \hat{b}}{ }^{i j}, \\
& \hat{\mathscr{R}}(M)_{\hat{a} \hat{b}} \hat{c}^{\hat{d}}=-\frac{1}{4}\left(\Sigma_{\hat{a} \hat{b}}\right)^{\hat{\alpha} \hat{\beta}}\left(\sum^{\hat{c} \hat{d}}\right)^{\hat{\gamma} \hat{\delta}}\left(\mathrm{i} W_{\hat{\alpha} \hat{\beta} \hat{\gamma} \hat{\delta}}+3 W_{(\hat{\alpha} \hat{\beta}} W_{\hat{\gamma} \hat{\delta})}\right), \\
& \hat{\mathscr{R}}(S)_{\hat{a} \hat{b} \hat{\alpha}}^{i}=-\frac{1}{2} \hat{\nabla}_{\hat{\alpha}}^{\hat{\beta}} W_{\hat{a} \hat{b} \hat{\beta}}^{i}-\frac{1}{2}\left(\Gamma_{[\hat{a}}\right)_{\hat{\alpha}}^{\hat{\beta}} \hat{\nabla}^{\hat{c}} W_{\hat{b}] \hat{c} \hat{\beta}}{ }^{i} \\
& -\frac{1}{8} W_{\hat{\alpha}}^{\hat{\beta}} W_{\hat{a} \hat{b} \hat{\beta}}^{i}+\frac{1}{16}\left(\Sigma_{\hat{a} \hat{b}}\right)_{\hat{\alpha}}^{\hat{\beta}} W^{\hat{c} \hat{d}} W_{\hat{c} \hat{d} \hat{\beta}}{ }^{i}+\frac{3}{8} W_{[\hat{a}}^{\hat{c}} W_{\hat{b}] \hat{c} \hat{\alpha}}{ }^{i}, \\
& \hat{\mathscr{R}}(K)_{\hat{a} \hat{b}}^{\hat{c}}=\frac{1}{4} \hat{\nabla}_{d} \hat{\mathscr{R}}(M)_{\hat{a} \hat{b}}{ }_{\hat{c}}^{\hat{d}}-\frac{\mathrm{i}}{16} W_{\hat{a} b j}^{\hat{\alpha}}\left(\Gamma^{\hat{c}}\right)_{\hat{\alpha}}^{\hat{\beta}} X_{\hat{\beta}}^{j}-\frac{\mathrm{i}}{8} W_{\hat{d}[\hat{a} j}^{\hat{\alpha} j}\left(\Gamma_{\hat{b}]}\right)_{\hat{\alpha}}^{\hat{\beta}} W_{\hat{\beta}}^{\hat{c} \hat{j} j} \\
& +\frac{\mathrm{i}}{8} W_{\hat{a} \hat{d}^{i}}^{\hat{\alpha}}\left(\Gamma^{\hat{c}}\right)_{\hat{\alpha}}^{\hat{\beta}} W_{\hat{b} \hat{\beta}}^{\hat{d}}{ }^{i} \text {. }
\end{aligned}
$$

\section{Conventions for 5D conformal supergravity}

For the convenience of the reader, we provide in table 1 a brief translation scheme between our conventions and the other groups'. A similar table may be found in [32]. 


\begin{tabular}{|c|c|c|c|}
\hline Our conventions & de Wit and Katmadas & Bergshoeff et al. & Fujita et al. \\
\hline$\eta^{\hat{a} \hat{b}}$ & $\eta^{a b}$ & $\eta^{a b}$ & $-\eta^{a b}$ \\
\hline$\Gamma^{\hat{a}}$ & $-\mathrm{i} \gamma^{a}$ & $\mathrm{i} \gamma^{a}$ & $\gamma^{a}$ \\
\hline$\sum^{\hat{a} \hat{b}}$ & $\frac{1}{2} \gamma^{a b}$ & $\frac{1}{2} \gamma^{a b}$ & $-\frac{1}{2} \gamma^{a b}$ \\
\hline$\varepsilon^{\hat{a} \hat{b} \hat{b} \hat{d} \hat{e}}$ & $-\mathrm{i} \varepsilon^{a b c d e}$ & $-\varepsilon^{a b c d e}$ & $\varepsilon^{a b c d e}$ \\
\hline$\psi_{\hat{m}}^{i}$ & $\psi_{\mu}^{i}$ & $\psi_{\mu}^{i}$ & $2 \psi_{\mu}^{i}$ \\
\hline $\mathcal{V}_{\hat{m}}{ }^{i}{ }_{j}$ & $-\frac{1}{2} V_{\mu j}{ }^{i}$ & $-V_{\mu}{ }^{i}{ }_{j}$ & $V_{\mu}{ }^{i}{ }_{j}$ \\
\hline$\hat{\omega}_{\hat{m}}^{\hat{a} \hat{b}}$ & $\omega_{\mu}^{a b}$ & $-\omega_{\mu}^{a b}$ & $-\omega_{\mu}^{a b}$ \\
\hline $\mathrm{i} \hat{\phi}_{\hat{m}}^{i}$ & $\phi_{\mu}^{i}$ & $-\phi_{\mu}{ }^{i}+\frac{1}{3} T_{a b} \gamma^{a b} \psi_{\mu}{ }^{i}$ & $2 \phi_{\mu}^{i}-\frac{2}{3} v_{a b} \gamma^{a b} \psi_{\mu}^{i}$ \\
\hline$\hat{f}_{\hat{m}} \hat{a}$ & $-f_{\mu}^{a}+\frac{1}{3} \psi_{\mu i} \gamma^{a} \chi^{i}$ & $-f_{\mu}^{a}+\frac{1}{3} \psi_{\mu}^{i} \gamma^{a} \chi_{i}$ & $-f_{\mu}^{a}+\frac{\mathrm{i}}{24} \psi_{\mu}^{i} \gamma^{a} \chi_{i}$ \\
\hline$w^{\hat{a} \hat{b}}$ & $-4 T^{a b}$ & $\frac{16}{3} T^{a b}$ & $\frac{4}{3} v^{a b}$ \\
\hline$\chi^{i}$ & $\chi^{i}$ & $\chi^{i}$ & $\frac{1}{32} \chi^{i}$ \\
\hline$D$ & $D$ & $D$ & $\frac{1}{16}\left(D-\frac{8}{3} v^{a b} v_{a b}\right)$ \\
\hline$\hat{R}(Q)_{\hat{a} \hat{b}}^{i}$ & $\frac{1}{2} R(Q)_{a b}^{i}$ & $\frac{1}{2} \hat{R}(Q)_{a b}^{i}$ & $\hat{R}(Q)_{a b}^{i}$ \\
\hline$\hat{R}(M)_{\hat{a} \hat{b}}^{\hat{b} \hat{d}}$ & $R(M)_{a b}^{c d}$ & $-\hat{R}(M)_{a b}{ }^{c d}$ & $-\hat{R}(M)_{a b}{ }^{c d}$ \\
\hline$\hat{R}(J)_{\hat{a} \hat{b}{ }_{j}^{i}}$ & $-\frac{1}{2} R(\mathcal{V})_{a b j}^{i}$ & $-\hat{R}(V)_{a b}{ }^{i}{ }_{j}$ & $\hat{R}(U)_{a b}{ }^{i}{ }_{j}$ \\
\hline $\mathrm{i} \hat{R}(S)_{\hat{a} \hat{b}}^{i}$ & $\frac{1}{2} R(S)_{a b}^{i}$ & $-\frac{1}{2} \hat{R}(S)_{a b}{ }^{i}+\frac{1}{6} T_{c d} \gamma^{c d} \hat{R}(Q)_{a b}^{i}$ & $\hat{R}(S)_{a b}{ }^{i}-\frac{1}{3} v_{c d} \gamma^{c d} \hat{R}(Q)_{a b}{ }^{i}$ \\
\hline$\hat{R}(K)_{\hat{a} \hat{b}}^{\hat{c}}$ & $-R(K)_{a b}{ }^{c}+\frac{1}{3} R(Q)_{a b i} \gamma^{c} \chi^{i}$ & $-\hat{R}(K)_{a b}{ }^{c}+\frac{1}{3} \hat{R}(Q)_{a b^{i}} \gamma^{c} \chi_{i}$ & $-\hat{R}(K)_{a b}{ }^{c}+\frac{\mathrm{i}}{24} \hat{R}(Q)_{a b}{ }^{i} \gamma^{c} \chi_{i}$ \\
\hline
\end{tabular}

Table 1. Conventions for Weyl multiplet.

\begin{tabular}{|ccc|}
\hline de Wit and Katmadas & Bergshoeff et al. & Fujita et al. \\
\hline$\varepsilon^{i}=2 \xi^{i}$ & $\varepsilon^{i}=2 \xi^{i}$ & $\varepsilon^{i}=\xi^{i}$ \\
$\eta^{i}=2 \mathrm{i} \eta^{i}$ & $\eta^{\prime i}=-2 \mathrm{i} \eta^{i}+\frac{2}{3} T_{a b} \gamma^{a b} \xi^{i}$ & $\eta^{i}=\mathrm{i} \eta^{i}+\frac{1}{3} v_{a b} \gamma^{a b} \xi^{i}$ \\
$\Lambda_{K}^{\prime a}=-\Lambda_{K}^{a}+\frac{2}{3} \xi_{i} \gamma^{a} \chi^{i}$ & $\Lambda_{K}^{\prime a}=-\Lambda_{K}^{a}+\frac{2}{3} \xi^{i} \gamma^{a} \chi_{i}$ & $\Lambda_{K}^{a}=-\Lambda_{K}^{a}+\frac{\mathrm{i}}{24} \xi^{i} \gamma^{a} \chi_{i}$ \\
\hline
\end{tabular}

Table 2. Conventions for $\delta_{Q}+\delta_{S}+\delta_{K}$.

We must be careful to note that the definitions of supersymmetry are different between the various groups, with the differences amounting not only to normalizations but also to additional field-dependent $S$ and $K$ transformations in the definition of $\delta_{Q}$. In other words, given a transformation $\delta_{Q}+\delta_{S}+\delta_{K}$ in our conventions with respective parameters $\xi_{\hat{\alpha}}^{i}, \eta_{\hat{\alpha}}^{i}$ and $\Lambda_{K}^{\hat{a}}$, we will find a transformation $\delta_{Q}^{\prime}+\delta_{S}^{\prime}+\delta_{K}^{\prime}$ with new parameters $\varepsilon^{i}, \eta^{i}$ and $\Lambda_{K}^{\prime a}$ given in table 2 .

It should be emphasized that each group uses the same vector derivative $D_{a}$, corresponding to our $\hat{\nabla}_{\hat{a}}$, modulo differing overall normalizations of the superconformal generators. The additional gravitino-dependent terms in the $S$-supersymmetry and special conformal connections in table 1 cancel against additional terms found within $\delta_{Q}$, so that the vector derivative is unchanged. 


\begin{tabular}{|cccc|}
\hline Our conventions & de Wit and Katmadas & Bergshoeff et al. & Fujita et al. \\
\hline$W$ & $\sigma$ & $-\sigma$ & $M$ \\
$\lambda^{i}$ & $\Omega^{i}$ & $\psi^{i}$ & $-2 \Omega^{i}$ \\
$X^{i j}$ & $2 Y^{i j}$ & $-2 Y^{i j}$ & $2 Y^{i j}$ \\
\hline
\end{tabular}

Table 3. Conventions for vector multiplet.

For completeness, we also give in table 3 the relation between our conventions for the vector multiplet and the other groups.

\section{E The $\mathcal{O}(2)$ multiplet prepotential from harmonic superspace}

In this appendix we use the harmonic superspace techniques [59] extended to the $5 \mathrm{D} \mathcal{N}=1$ super-Poincaré case (see $[2,51]$ for the technical details regarding the $\mathcal{N}=1$ harmonic superspace in five dimensions) to derive a prepotential formulation for the $\mathcal{O}(2)$ multiplet. In appendix $\mathrm{G}$, the same techniques will be used to derive unconstrained prepotentials for the $\mathcal{O}(4+n)$ multiplets, $n=0,1, \ldots$, in $5 \mathrm{D} \mathcal{N}=1$ Minkowski superspace. ${ }^{40}$

We consider an $\mathcal{O}(2)$ multiplet $G^{i j}(z)$ in $5 \mathrm{D} \mathcal{N}=1$ Minkowski superspace and associate with it the analytic superfield $G^{++}\left(z, u^{+}\right)=G^{i j}(z) u_{i}^{+} u_{j}^{+}$. The latter is constrained by

$$
D_{\hat{\alpha}}^{+} G^{++}=0, \quad D^{++} G^{++}=0,
$$

where $D^{++}:=u^{+i} \partial / \partial u^{-i}$. As in the $4 \mathrm{D} \mathcal{N}=2$ super-Poincaré case [101], the analytic projector on the space of $\mathcal{O}(2)$ multiplets $^{41}$ is

$$
\Pi_{\mathrm{L}}^{(2,2)}\left(\zeta_{1}, \zeta_{2}\right)=-\left(\hat{D}_{1}^{+}\right)^{4}\left(\hat{D}_{2}^{+}\right)^{4} \frac{1}{\square} \frac{\delta^{5 \mid 8}\left(z_{1}-z_{2}\right)}{\left(u_{1}^{+} u_{2}^{+}\right)^{2}},
$$

where

$$
\left(\hat{D}^{+}\right)^{4}=-\frac{1}{32}\left(\hat{D}^{+}\right)^{2}\left(\hat{D}^{+}\right)^{2}, \quad\left(\hat{D}^{+}\right)^{2}=D^{+\hat{\alpha}} D_{\hat{\alpha}}^{+},
$$

and $\zeta$ denotes the coordinates of the analytic subspace. The properties of $\Pi_{\mathrm{T}}^{(2,2)}\left(\zeta_{1}, \zeta_{2}\right)$ are:

$$
\begin{aligned}
D_{1}^{+\hat{\alpha}} \Pi_{\mathrm{T}}^{(2,2)}\left(\zeta_{1}, \zeta_{2}\right) & =D_{2}^{+\hat{\alpha}} \Pi_{\mathrm{T}}^{(2,2)}\left(\zeta_{1}, \zeta_{2}\right)=0, \\
D_{1}^{++} \Pi_{\mathrm{T}}^{(2,2)}\left(\zeta_{1}, \zeta_{2}\right) & =D_{2}^{++} \Pi_{\mathrm{T}}^{(2,2)}\left(\zeta_{1}, \zeta_{2}\right)=0, \\
\int \mathrm{d} \zeta_{3}^{(-4)} \Pi_{\mathrm{T}}^{(2,2)}\left(\zeta_{1}, \zeta_{3}\right) \Pi_{\mathrm{T}}^{(2,2)}\left(\zeta_{3}, \zeta_{2}\right) & =\Pi_{\mathrm{T}}^{(2,2)}\left(\zeta_{1}, \zeta_{2}\right), \\
\left(\Pi_{\mathrm{T}}^{(2,2)}\left(\zeta_{1}, \zeta_{2}\right)\right)^{\mathrm{T}} & =\Pi_{\mathrm{T}}^{(2,2)}\left(\zeta_{2}, \zeta_{1}\right) .
\end{aligned}
$$

\footnotetext{
${ }^{40}$ The harmonic and projective superspace descriptions of the $\mathcal{O}(n)$ multiplets are completely equivalent [100].

${ }^{41}$ This projector plays an important role in computing the one-loop effective action for $\mathcal{N}=4 \mathrm{SYM}$ in four dimensions [102].
} 
For any $\mathcal{O}(2)$ multiplet $G^{++}$we have

$$
G^{++}\left(z_{1}, u_{1}^{+}\right) \equiv G^{++}\left(\zeta_{1}\right)=\int \mathrm{d} \zeta_{2}^{(-4)} \Pi_{\mathrm{L}}^{(2,2)}\left(\zeta_{1}, \zeta_{2}\right) G^{++}\left(\zeta_{2}\right)
$$

Introduce a superfield $\Xi^{--}(z, u)$ such that $\left(\hat{D}^{+}\right)^{4} \Xi^{--}=G^{++}$. Then we can rewrite (E.5) as follows

$$
G^{++}\left(\zeta_{1}\right)=\int \mathrm{d}^{5 \mid 8} z_{2} \mathrm{~d} u_{2} \Pi_{\mathrm{L}}^{(2,2)}\left(\zeta_{1}, \zeta_{2}\right) \Xi^{--}\left(z_{2}, u_{2}\right) .
$$

In the expression (E.2) we represent

$$
\begin{aligned}
\left(\hat{D}_{2}^{+}\right)^{4} \delta^{5 \mid 8}\left(z_{1}-z_{2}\right) & =-\frac{1}{32}\left(\hat{D}_{2}^{+}\right)^{2}\left(\hat{D}_{2}^{+}\right)^{2} \delta^{5 \mid 8}\left(z_{1}-z_{2}\right) \\
& =-\frac{1}{32}\left(\hat{D}_{2}^{+}\right)^{2} u_{2}^{+i} u_{2}^{+j} \hat{D}_{2 i j} \delta^{5 \mid 8}\left(z_{1}-z_{2}\right) \\
& =-\frac{1}{32}\left(\hat{D}_{2}^{+}\right)^{2} u_{2}^{+i} u_{2}^{+j} \hat{D}_{1 i j} \delta^{5 \mid 8}\left(z_{1}-z_{2}\right) \\
& =-\frac{1}{32} u_{2}^{+i} u_{2}^{+j} \hat{D}_{1 i j}\left(\hat{D}_{2}^{+}\right)^{2} \delta^{5 \mid 8}\left(z_{1}-z_{2}\right) .
\end{aligned}
$$

We plug this expression in (E.2) and make use of the identity

$$
\Psi_{2}^{+}=\left(u_{1}^{+} u_{2}^{+}\right) \Psi_{1}^{-}-\left(u_{1}^{-} u_{2}^{+}\right) \Psi_{1}^{+}, \quad \Psi^{ \pm}=\Psi^{i} u_{i}^{ \pm}
$$

in conjunction with $D_{1}^{\hat{\alpha}+}\left(\hat{D}_{1}^{+}\right)^{4}=\left(\hat{D}_{1}^{+}\right)^{4} D_{1}^{\hat{\alpha}+}=0$. This gives

$$
\Pi_{\mathrm{L}}^{(2,2)}\left(\zeta_{1}, \zeta_{2}\right)=\frac{1}{32}\left(\hat{D}_{1}^{+}\right)^{4}\left(\hat{D}_{1}^{-}\right)^{2}\left(\hat{D}_{2}^{+}\right)^{2} \frac{1}{\square} \delta^{5 \mid 8}\left(z_{1}-z_{2}\right) .
$$

As a result, relation (E.6) becomes equivalent to

$$
G^{++}\left(z, u^{+}\right)=\left(\hat{D}^{+}\right)^{4}\left(\hat{D}^{-}\right)^{2} \Omega(z) .
$$

\section{F Gauge freedom for the $\mathcal{O}(2)$ multiplet}

Let us show that the gauge transformation of the $\mathcal{O}(2)$ multiplet prepotential $\Omega$, eq. (7.10), leaves invariant the superfield $G^{(2)}$ defined by (7.5). We need to prove that the superfield

$$
\Omega(B)=-\frac{\mathrm{i}}{2} \nabla_{\hat{\alpha}}^{k} \nabla_{\hat{\beta}}^{l} B_{k l}^{\hat{\alpha} \hat{\beta}}, \quad B_{\hat{\alpha} \hat{\beta}}^{i j}=\left(\Gamma^{\hat{a}}\right)_{\hat{\alpha} \hat{\beta}} B_{\hat{a}}^{i j},
$$

is annihilated by the operator $\mathrm{i} \Delta^{i j k l} \nabla_{k l}$. It is useful to employ the equivalent expression for $\Omega(B)$ given by

$$
\begin{aligned}
\Omega(B)= & -\frac{\mathrm{i}}{2} \nabla_{\hat{\alpha}}^{(1)}\left(\nabla_{\hat{\beta}}^{(1)} B^{\hat{\alpha} \hat{\beta}(-2)}-2 \nabla_{\hat{\beta}}^{(-1)} B^{\hat{\alpha} \hat{\beta}(0)}\right) \\
& -\frac{\mathrm{i}}{2} \nabla_{\hat{\alpha}}^{(-1)} \nabla_{\hat{\beta}}^{(-1)} B^{\hat{\alpha} \hat{\beta}(2)}+\nabla_{\hat{\alpha} \hat{\beta}} B^{\hat{\alpha} \hat{\beta}(0)} \\
B_{\hat{a}}^{(2)}:= & v_{i} v_{j} B_{\hat{a}}^{i j}, \quad B_{\hat{a}}^{(0)}:=\frac{v_{i} u_{j}}{(v, u)} B_{\hat{a}}^{i j}, \quad B_{\hat{a}}^{(-2)}:=\frac{u_{i} u_{j}}{(v, u)^{2}} B_{\hat{a}}{ }^{i j} .
\end{aligned}
$$


By using

$$
\nabla^{(-2)} \nabla_{\hat{\alpha}}^{(-1)} \nabla_{\hat{\beta}}^{(-1)}=-\frac{1}{4} \varepsilon_{\hat{\alpha} \hat{\beta}} \nabla^{(-2)} \nabla^{(-2)},
$$

and $\Delta^{(4)} \nabla_{\hat{\alpha}}^{(1)}=0$, we obtain

$$
\begin{aligned}
\mathrm{i} \Delta^{(4)} \nabla^{(-2)} \Omega(B)= & -\frac{1}{2} \Delta^{(4)}\left[\nabla_{\hat{\alpha}}^{(1)}, \nabla^{(-2)}\right]\left(\nabla_{\hat{\beta}}^{(1)} B^{\hat{\alpha} \hat{\beta}(-2)}-2 \nabla_{\hat{\beta}}^{(-1)} B^{\hat{\alpha} \hat{\beta}(0)}\right) \\
& +\mathrm{i} \Delta^{(4)} \nabla^{(-2)} \nabla_{\hat{\alpha} \hat{\beta}} B^{\hat{\alpha} \hat{\beta}(0)} .
\end{aligned}
$$

By making use of

$$
\left[\nabla_{\hat{\alpha} \hat{\beta}}, \nabla_{\hat{\gamma}}^{j}\right]=\left(\Gamma^{\hat{a}}\right)_{\hat{\alpha} \hat{\beta}}\left(\Gamma_{\hat{a}}\right)_{\hat{\gamma} \hat{\delta}}\left[\mathscr{W}, \nabla^{\hat{\delta} j}\right], \quad\left[\nabla_{\hat{\alpha} \hat{\beta}}, \nabla^{\hat{\beta} j}\right]=-5\left[\mathscr{W}, \nabla_{\hat{\alpha}}^{j}\right],
$$

it can be seen that

$$
\left[\nabla_{\hat{\alpha}}^{(1)}, \nabla^{(-2)}\right]=-4 \mathrm{i}\left(\nabla^{\hat{\gamma}(-1)} \nabla_{\hat{\alpha} \hat{\gamma}}+\nabla_{\hat{\alpha}}^{(-1)} \mathscr{W}-2\left[\mathscr{W}, \nabla_{\hat{\alpha}}^{(-1)}\right]\right) .
$$

Note that in performing this calculation we will keep implicit as long as possible the expression (2.30) for the operator $\mathscr{W}$ in the covariant derivative algebra (2.27). Plugging eq. (F.6) into (F.4), after some algebra one can obtain

$$
\begin{aligned}
& \mathrm{i} \Delta^{(4)} \nabla^{(-2)} \Omega(B)=\mathrm{i} \Delta^{(4)}\left\{\operatorname{tr}\left[\Gamma^{\hat{a}} \Gamma^{\hat{b}} \Gamma^{\hat{c}}\right]\left(4 \mathrm{i} \nabla_{\hat{a}} \nabla_{\hat{b}} B_{\hat{c}}^{(-2)}-\left(\Gamma_{\hat{b}}\right)^{\hat{\gamma}^{\prime} \hat{\beta}^{\prime}} \nabla_{\hat{\gamma}^{\prime}}^{(-1)} \nabla_{\hat{\beta}^{\prime}}^{(-1)} \nabla_{\hat{a}} B_{\hat{c}}^{(0)}\right)\right. \\
& -\frac{1}{2} \operatorname{tr}\left[\Gamma^{\hat{a}} \Gamma^{\hat{b}} \Gamma_{\hat{a}}\right] \nabla^{\hat{\rho}(-1)}\left(\left[\mathscr{W}, \nabla_{\hat{\rho}}^{(1)}\right] B_{\hat{b}}^{(-2)}-2\left[\mathscr{W}, \nabla_{\hat{\rho}}^{(-1)}\right] B_{\hat{b}}^{(0)}\right) \\
& -\frac{1}{2} \operatorname{tr}\left[\Gamma^{\hat{a}} \Gamma^{\hat{b}} \Gamma_{\hat{a}} \Gamma^{\hat{c}}\right]\left(\Gamma_{\hat{b}}\right) \hat{\rho} \hat{\tau} \nabla^{\hat{\rho}(-1)}\left(\left[\mathscr{W}, \nabla^{\hat{\tau}(1)}\right] B_{\hat{c}}^{(-2)}-2\left[\mathscr{W}, \nabla^{\hat{\tau}(-1)}\right] B_{\hat{c}}^{(0)}\right) \\
& -4 \mathrm{i}\left[\mathscr{W}, \nabla^{\hat{\alpha} \hat{\beta}}\right] B_{\hat{\alpha} \hat{\beta}}^{(-2)}-8 \mathrm{i} \nabla^{\hat{\alpha} \hat{\beta}} \mathscr{W} B_{\hat{\alpha} \hat{\beta}}^{(-2)}+2 \nabla_{\hat{\alpha}}^{(-1)}\left[\mathscr{W}, \nabla_{\hat{\beta}}^{(1)}\right] B^{\hat{\alpha} \hat{\beta}(-2)} \\
& -4 \nabla_{\hat{\alpha}}^{(-1)} \nabla_{\hat{\beta}}^{(-1)} \mathscr{W} B^{\hat{\alpha} \hat{\beta}(0)}-4\left\{\nabla_{\hat{\alpha}}^{(1)},\left[\nabla_{\hat{\beta}}^{(-1)}, \mathscr{W}\right]\right\} B^{\hat{\alpha} \hat{\beta}(-2)} \\
& \left.+4 \nabla_{\hat{\alpha}}^{(-1)}\left[\mathscr{W}, \nabla_{\hat{\beta}}^{(-1)}\right] B^{\hat{\alpha} \hat{\beta}(0)}-8\left\{\left[\mathscr{W}, \nabla_{\hat{\alpha}}^{(-1)}\right], \nabla_{\hat{\beta}}^{(-1)}\right\} B^{\hat{\alpha} \hat{\beta}(0)}\right\} .
\end{aligned}
$$

Some terms in the previous expression are identically zero. First of all note that due to (A.21b) we have

$$
\operatorname{tr}\left[\Gamma_{\hat{a}} \Gamma_{\hat{b}} \Gamma_{\hat{c}}\right]=0 .
$$

Then the first two lines in (F.7) are zero. Moreover, the Bianchi identity (2.28) implies

$$
\left(\Gamma_{\hat{a}}\right)^{\hat{\alpha} \hat{\beta}}\left\{\left[\mathscr{W}, \nabla_{\hat{\alpha}}^{(-1)}\right], \nabla_{\hat{\beta}}^{(-1)}\right\}=0,
$$

which removes the last term in (F.7). Once we use

$$
\operatorname{tr}\left[\Gamma^{\hat{a}} \Gamma^{\hat{b}} \Gamma^{\hat{c}} \Gamma^{\hat{d}}\right]=4\left(\eta^{\hat{a} \hat{b}} \eta^{\hat{c} \hat{d}}-\eta^{\hat{a} \hat{c}} \eta^{\hat{b} \hat{d}}+\eta^{\hat{a} \hat{d}} \eta^{\hat{b} \hat{c}}\right), \quad \operatorname{tr}\left[\Gamma^{\hat{a}} \Gamma^{\hat{b}} \Gamma_{\hat{a}} \Gamma^{\hat{c}}\right]=-12 \eta^{\hat{b} \hat{c}}
$$

which follow from (A.21c), (F.7) can be brought to the following form:

$$
\begin{aligned}
\mathrm{i} \Delta^{(4)} \nabla^{(-2)} \Omega(B)=\mathrm{i} \Delta^{(4)}\left\{-4 \mathrm{i}\left[\mathscr{W}, \nabla^{\hat{\alpha} \hat{\beta}}\right] B_{\hat{\alpha} \hat{\beta}}^{(-2)}-8 \mathrm{i} \nabla^{\hat{\alpha} \hat{\beta}} \mathscr{W} B_{\hat{\alpha} \hat{\beta}}^{(-2)}\right. \\
+8 \nabla_{\hat{\alpha}}^{(-1)}\left[\mathscr{W}, \nabla_{\hat{\beta}}^{(1)}\right] B^{\hat{\alpha} \hat{\beta}(-2)}-4\left\{\nabla_{\hat{\alpha}}^{(1)},\left[\nabla_{\hat{\beta}}^{(-1)}, \mathscr{W}\right]\right\} B^{\hat{\alpha} \hat{\beta}(-2)}
\end{aligned}
$$




$$
\left.-8 \nabla_{\hat{\alpha}}^{(-1)}\left[\mathscr{W}, \nabla_{\hat{\beta}}^{(-1)}\right] B^{\hat{\alpha} \hat{\beta}(0)}-4 \nabla_{\hat{\alpha}}^{(-1)} \nabla_{\hat{\beta}}^{(-1)} \mathscr{W} B^{\hat{\alpha} \hat{\beta}(0)}\right\} .
$$

As a next step, we can simplify the second term in the second line. In fact, the Bianchi identity (2.28) implies

$$
\left(\Gamma_{\hat{a}}\right)^{\hat{\alpha} \hat{\beta}}\left\{\nabla_{\hat{\alpha}}^{(1)},\left[\nabla_{\hat{\beta}}^{(-1)}, \mathscr{W}\right]\right\}=-\left(\Gamma_{\hat{a}}\right)^{\hat{\alpha} \hat{\beta}}\left\{\nabla_{\hat{\alpha}}^{(-1)},\left[\nabla_{\hat{\beta}}^{(1)}, \mathscr{W}\right]\right\},
$$

which together with the super-Jacobi identity, can be used to derive the following result

$$
\left(\Gamma_{\hat{a}}\right)^{\hat{\alpha} \hat{\beta}}\left\{\nabla_{\hat{\alpha}}^{(1)},\left[\nabla_{\hat{\beta}}^{(-1)}, \mathscr{W}\right]\right\}=-\frac{1}{2}\left(\Gamma_{\hat{a}}\right)^{\hat{\alpha} \hat{\beta}}\left[\mathscr{W},\left\{\nabla_{\hat{\alpha}}^{(1)}, \nabla_{\hat{\beta}}^{(-1)}\right\}\right]=-\mathrm{i}\left(\Gamma_{\hat{a}}\right)^{\hat{\alpha} \hat{\beta}}\left[\mathscr{W}, \nabla_{\hat{\alpha} \hat{\beta}}\right]
$$

If we use this expression in (F.11), we arrive at the simple result

$$
\begin{aligned}
\mathrm{i} \Delta^{(4)} \nabla^{(-2)} \Omega(B)=\mathrm{i} \Delta^{(4)}\{ & 8 \nabla_{\hat{\alpha}}^{(-1)}\left[\mathscr{W}, \nabla_{\hat{\beta}}^{(1)}\right] B^{\hat{\alpha} \hat{\beta}(-2)}-8 \nabla_{\hat{\alpha}}^{(-1)}\left[\mathscr{W}, \nabla_{\hat{\beta}}^{(-1)}\right] B^{\hat{\alpha} \hat{\beta}(0)} \\
& \left.+4 \nabla_{\hat{\alpha}}^{(-1)} \nabla_{\hat{\beta}}^{(1)} \mathscr{W} B^{\hat{\alpha} \hat{\beta}(-2)}-4 \nabla_{\hat{\alpha}}^{(-1)} \nabla_{\hat{\beta}}^{(-1)} \mathscr{W} B^{\hat{\alpha} \hat{\beta}(0)}\right\} \\
\mathrm{i} \Delta^{(4)} \nabla^{(-2)} \Omega(B)=- & \frac{4 \mathrm{i} u_{i} u_{j}}{(v, u)^{2}} \Delta^{(4)}\left\{2 \nabla_{\hat{\alpha}}^{(i}\left[\mathscr{W}, \nabla_{\hat{\beta} k}\right] B^{\hat{\alpha} \hat{\beta} j) k}+\nabla_{\hat{\alpha}}^{(i} \nabla_{\hat{\beta} k} \mathscr{W} B^{\hat{\alpha} \hat{\beta} j) k}\right\} .
\end{aligned}
$$

Now we use the explicit expression of $\mathscr{W}$ and obtain

$$
\begin{aligned}
\mathscr{W} B^{\hat{\alpha} \hat{\beta} i j} & =2 W^{[\hat{\alpha}}{ }_{\hat{\gamma}} B^{\hat{\beta}] \hat{\gamma} i j}, \\
{\left[\mathscr{W}, \nabla_{\hat{\beta} k}\right] B^{\hat{\alpha} \hat{\beta} j k} } & =-W_{\hat{\beta} \hat{\delta}} \nabla_{k}^{\hat{\delta}} B^{\hat{\alpha} \hat{\beta} j k}-5 X_{\hat{\beta} k} B^{\hat{\alpha} \hat{\beta} j k}=-\nabla_{k}^{\hat{\gamma}} W_{\hat{\gamma} \hat{\beta}} B^{\hat{\alpha} \hat{\beta} j k} .
\end{aligned}
$$

Equation (F.14) then becomes

$$
\begin{aligned}
& \mathrm{i} \Delta^{(4)} \nabla^{(-2)} \Omega(B)=-\frac{8 \mathrm{i} u_{i} u_{j}}{(v, u)^{2}} \Delta^{(4)} \nabla_{\hat{\alpha}}^{i} \nabla_{\hat{\beta} k} W^{(\hat{\alpha}} B^{\hat{\beta}) \hat{\gamma} j k} \\
& \mathrm{i} \Delta^{(4)} \nabla^{(-2)} \Omega(B)=8 \mathrm{i} \Delta^{(4)} \nabla_{(\hat{\alpha}}^{(1)} \nabla_{\hat{\beta})}^{(-1)} W_{\hat{\gamma}}^{(\hat{\alpha}} B^{\hat{\beta}) \hat{\gamma}(-2)} \equiv 0 .
\end{aligned}
$$

This completes the proof that the operator $\Delta^{(4)} \nabla^{(-2)}$ annihilates the superfield (F.1).

\section{G Prepotentials for $\mathcal{O}(4+n)$ multiplets, $n=0,1, \ldots$, from harmonic superspace}

Here we consider an $\mathcal{O}(4)$ multiplet $G^{(4)}(z, u)=G^{i j k l}(z) u_{i}^{+} u_{j}^{+} u_{k}^{+} u_{l}^{+}$realized in $5 \mathrm{D} \mathcal{N}=1$ harmonic superspace,

$$
D_{\hat{\alpha}}^{+} G^{(4)}=0, \quad D^{++} G^{(4)}=0 .
$$

It may be represented as

$$
G^{(4)}(u)=\left(\hat{D}^{+}\right)^{4} V(u)
$$

where

$$
V(u)=V_{0}+\sum_{n=1}^{\infty} V^{\left(i_{1} \ldots i_{2 n}\right)} u_{i_{1}}^{+} \ldots u_{i_{n}}^{+} u_{i_{n+1}}^{-} \ldots u_{i_{2 n}}^{-} \equiv V_{0}+\mathfrak{V}(u)
$$

obeys the equation

$$
D^{++} V=D^{++} \mathfrak{V}=D^{+\hat{\alpha}} \Sigma_{\hat{\alpha}}^{+}
$$


for some spinor superfield $\Sigma_{\hat{\alpha}}^{+}(u)$. We note that $V(u)$ is defined modulo abelian gauge transformations of the form:

$$
V \rightarrow \widetilde{V}=\widetilde{V}_{0}+\widetilde{\mathfrak{V}}:=V+D^{+\hat{\alpha}} \lambda_{\hat{\alpha}}^{-},
$$

where $\lambda_{\hat{\alpha}}^{-}(u)$ is arbitrary. We now consider the following harmonic equation

$$
D^{++} \lambda_{\hat{\alpha}}^{-}=-\Sigma_{\hat{\alpha}}^{+},
$$

with $\Sigma_{\hat{\alpha}}^{+}$given. This equation proves to have a unique solution $\lambda_{\hat{\alpha}}^{-}(u)$. Upon applying the above gauge transformation, we obtain

$$
D^{++} \widetilde{V}=0 \Longrightarrow \tilde{V}=\widetilde{V}_{0} .
$$

As a result, the $\mathcal{O}(4)$ multiplet can always be represented in the form

$$
G^{(4)}(u)=\left(\hat{D}^{+}\right)^{4} V,
$$

with the prepotential $V$ being harmonic independent.

Given a non-negative integer $n=1,2, \ldots$, consider an $\mathcal{O}(4+n)$ multiplet

$$
G^{(4+n)}(z, u)=G^{i_{1} \ldots i_{4+n}}(z) u_{i_{1}}^{+} \ldots u_{i_{4+n}}^{+},
$$

realized in $5 \mathrm{D} \mathcal{N}=1$ harmonic superspace,

$$
D_{\hat{\alpha}}^{+} G^{(4+n)}=0, \quad D^{++} G^{(4+n)}=0 .
$$

The superfield $G^{(4+n)}$ may be represented as

$$
G^{(4+n)}(u)=\left(\hat{D}^{+}\right)^{4} V^{(n)}(u),
$$

where

$$
\begin{aligned}
V^{(n)}(u) & =V_{0}^{i_{1} \ldots i_{n}} u_{i_{1}}^{+} \ldots u_{i_{n}}^{+}+\sum_{m=1}^{\infty} V^{\left(i_{1} \ldots i_{n+2 m}\right)} u_{i_{1}}^{+} \ldots u_{i_{n+m}}^{+} u_{i_{n+m+1}}^{-} \ldots u_{i_{n+2 m}}^{-} \\
& \equiv V_{0}^{(n)}(u)+\mathfrak{V}^{(n)}(u)
\end{aligned}
$$

obeys the equation

$$
D^{++} V^{(n)}=D^{++} \mathfrak{V}^{(n)}=D^{+\hat{\alpha}} \Sigma_{\hat{\alpha}}^{(n+1)},
$$

for some harmonic superfield $\Sigma_{\hat{\alpha}}^{(n+1)}(u)$. By construction, the prepotential $V^{(n)}$ is defined modulo gauge transformations

$$
V^{(n)} \rightarrow \widetilde{V}^{(n)}=\widetilde{V}_{0}^{(n)}+\widetilde{\mathfrak{V}}^{(n)}:=V^{(n)}+D^{+\hat{\alpha}} \lambda_{\hat{\alpha}}^{(n-1)},
$$

for an arbitrary harmonic superfield $\lambda_{\hat{\alpha}}^{(n-1)}(u)$. It is possible to choose the gauge parameter $\lambda_{\hat{\alpha}}^{(n-1)}(u)$ to be a solution of the harmonic equation

$$
D^{++} \lambda_{\hat{\alpha}}^{(n-1)}=-\Sigma_{\hat{\alpha}}^{(n+1)} .
$$

Such a solution always exists and is not unique for $n>0$. Upon applying such a finite gauge transformation, we observe that the transformed prepotential $\widetilde{V}^{(n)}(u)$ is characterized by

$$
D^{++} \widetilde{\mathfrak{V}}^{(n)}=0 .
$$

We conclude that the $\mathcal{O}(4+n)$ multiplet can be represented in the form:

$$
G^{(4+n)}(u)=\left(\hat{D}^{+}\right)^{4} V^{(n)}(u), \quad V^{(n)}(u)=V^{i_{1} \ldots i_{n}} u_{i_{1}}^{+} \ldots u_{i_{n}}^{+} .
$$


Open Access. This article is distributed under the terms of the Creative Commons Attribution License (CC-BY 4.0), which permits any use, distribution and reproduction in any medium, provided the original author(s) and source are credited.

\section{References}

[1] J.P. Gauntlett, J.B. Gutowski, C.M. Hull, S. Pakis and H.S. Reall, All supersymmetric solutions of minimal supergravity in five-dimensions, Class. Quant. Grav. 20 (2003) 4587 [hep-th/0209114] [INSPIRE].

[2] S.M. Kuzenko and W.D. Linch III, On five-dimensional superspaces, JHEP 02 (2006) 038 [hep-th/0507176] [INSPIRE].

[3] S.M. Kuzenko and G. Tartaglino-Mazzucchelli, Five-dimensional $N=1$ AdS superspace: geometry, off-shell multiplets and dynamics, Nucl. Phys. B 785 (2007) 34 [arXiv:0704.1185] [INSPIRE].

[4] E. Cremmer, Supergravities in 5 dimensions, in Supergravity and superspace, S.W. Hawking and M. Roček eds., Cambridge University Press, Cambridge U.K. (1981), pg. 267 [INSPIRE].

[5] A.H. Chamseddine and $\mathrm{H}$. Nicolai, Coupling the $\mathrm{SO}(2)$ supergravity through dimensional reduction, Phys. Lett. B 96 (1980) 89 [INSPIRE].

[6] P.S. Howe, Off-shell $N=2$ and $N=4$ supergravity in five-dimensions, in Quantum structure of space and time, M.J. Duff and C.J. Isham eds., Cambridge University Press, Cambridge U.K. (1982), pg. 239.

[7] P.S. Howe and U. Lindström, The supercurrent in five-dimensions, Phys. Lett. B 103 (1981) 422 [INSPIRE].

[8] P. Breitenlohner and M.F. Sohnius, Superfields, auxiliary fields and tensor calculus for $N=2$ extended supergravity, Nucl. Phys. B 165 (1980) 483 [InSPIRE].

[9] P. Breitenlohner and M.F. Sohnius, An almost simple off-shell version of SU(2) Poincaré supergravity, Nucl. Phys. B 178 (1981) 151 [INSPIRE].

[10] M. Günaydin, G. Sierra and P.K. Townsend, The geometry of $N=2$ Maxwell-Einstein supergravity and Jordan algebras, Nucl. Phys. B 242 (1984) 244 [INSPIRE].

[11] M. Günaydin, G. Sierra and P.K. Townsend, Gauging the $D=5$ Maxwell-Einstein supergravity theories: more on Jordan algebras, Nucl. Phys. B 253 (1985) 573 [INSPIRE].

[12] M. Günaydin and M. Zagermann, The gauging of five-dimensional, $N=2$ Maxwell-Einstein supergravity theories coupled to tensor multiplets, Nucl. Phys. B 572 (2000) 131 [hep-th/9912027] [INSPIRE].

[13] A. Ceresole and G. Dall'Agata, General matter coupled $N=2, D=5$ gauged supergravity, Nucl. Phys. B 585 (2000) 143 [hep-th/0004111] [INSPIRE].

[14] M. Zucker, Minimal off-shell supergravity in five-dimensions, Nucl. Phys. B 570 (2000) 267 [hep-th/9907082] [INSPIRE].

[15] M. Zucker, Gauged N=2 off-shell supergravity in five-dimensions, JHEP 08 (2000) 016 [hep-th/9909144] [INSPIRE].

[16] M. Zucker, Off-shell supergravity in five-dimensions and supersymmetric brane world scenarios, Fortsch. Phys. 51 (2003) 899 [INSPIRE]. 
[17] T. Kugo and K. Ohashi, Supergravity tensor calculus in $5 D$ from $6 D$, Prog. Theor. Phys. 104 (2000) 835 [hep-ph/0006231] [INSPIRE].

[18] T. Kugo and K. Ohashi, Off-shell D = 5 supergravity coupled to matter Yang-Mills system, Prog. Theor. Phys. 105 (2001) 323 [hep-ph/0010288] [INSPIRE].

[19] T. Fujita and K. Ohashi, Superconformal tensor calculus in five-dimensions, Prog. Theor. Phys. 106 (2001) 221 [hep-th/0104130] [INSPIRE].

[20] T. Kugo and K. Ohashi, Gauge and nongauge tensor multiplets in $5 D$ conformal supergravity, Prog. Theor. Phys. 108 (2003) 1143 [hep-th/0208082] [INSPIRE].

[21] E. Bergshoeff et al., Weyl multiplets of $N=2$ conformal supergravity in five-dimensions, JHEP 06 (2001) 051 [hep-th/0104113] [INSPIRE].

[22] E. Bergshoeff et al., Superconformal $N=2, D=5$ matter with and without actions, JHEP 10 (2002) 045 [hep-th/0205230] [INSPIRE].

[23] E. Bergshoeff et al., $N=2$ supergravity in five-dimensions revisited, Class. Quant. Grav. 21 (2004) 3015 [Corrigendum ibid. 23 (2006) 7149] [hep-th/0403045] [INSPIRE].

[24] S.M. Kuzenko and G. Tartaglino-Mazzucchelli, Five-dimensional superfield supergravity, Phys. Lett. B 661 (2008) 42 [arXiv:0710.3440] [INSPIRE].

[25] S.M. Kuzenko and G. Tartaglino-Mazzucchelli, 5D supergravity and projective superspace, JHEP 02 (2008) 004 [arXiv:0712.3102] [INSPIRE].

[26] S.M. Kuzenko and G. Tartaglino-Mazzucchelli, Super-Weyl invariance in $5 D$ supergravity, JHEP 04 (2008) 032 [arXiv:0802.3953] [InSPIRE].

[27] W. Nahm, Supersymmetries and their representations, Nucl. Phys. B 135 (1978) 149 [INSPIRE].

[28] D.Z. Freedman and A. Van Proeyen, Supergravity, Cambridge University Press, Cambridge U.K. (2012).

[29] K. Hanaki, K. Ohashi and Y. Tachikawa, Supersymmetric completion of an $R^{2}$ term in five-dimensional supergravity, Prog. Theor. Phys. 117 (2007) 533 [hep-th/0611329] [INSPIRE].

[30] E.A. Bergshoeff, J. Rosseel and E. Sezgin, Off-shell $D=5, N=2$ Riemann squared supergravity, Class. Quant. Grav. 28 (2011) 225016 [arXiv:1107.2825] [INSPIRE].

[31] M. Ozkan and Y. Pang, Supersymmetric completion of Gauss-Bonnet combination in five dimensions, JHEP 03 (2013) 158 [Erratum ibid. 07 (2013) 152] [arXiv:1301.6622] [INSPIRE].

[32] M. Ozkan and Y. Pang, All off-shell $R^{2}$ invariants in five dimensional $N=2$ supergravity, JHEP 08 (2013) 042 [arXiv:1306.1540] [INSPIRE].

[33] S.M. Kuzenko, On compactified harmonic/projective superspace, $5 D$ superconformal theories and all that, Nucl. Phys. B 745 (2006) 176 [hep-th/0601177] [INSPIRE].

[34] S.M. Kuzenko, On superconformal projective hypermultiplets, JHEP 12 (2007) 010 [arXiv:0710.1479] [INSPIRE].

[35] A. Karlhede, U. Lindström and M. Roček, Selfinteracting tensor multiplets in $N=2$ superspace, Phys. Lett. B 147 (1984) 297 [INSPIRE]. 
[36] U. Lindström and M. Roček, New hyper-Kähler metrics and new supermultiplets, Commun. Math. Phys. 115 (1988) 21 [INSPIRE].

[37] U. Lindström and M. Roček, $N=2$ super Yang-Mills theory in projective superspace, Commun. Math. Phys. 128 (1990) 191 [inSPIRE].

[38] D. Butter, $N=1$ conformal superspace in four dimensions, Annals Phys. 325 (2010) 1026 [arXiv:0906.4399] [INSPIRE].

[39] D. Butter, $N=2$ conformal superspace in four dimensions, JHEP 10 (2011) 030 [arXiv:1103.5914] [INSPIRE].

[40] D. Butter, S.M. Kuzenko, J. Novak and G. Tartaglino-Mazzucchelli, Conformal supergravity in three dimensions: new off-shell formulation, JHEP 09 (2013) 072 [arXiv:1305.3132] [INSPIRE].

[41] P.A.M. Dirac, Wave equations in conformal space, Annals Math. 37 (1936) 429 [INSPIRE].

[42] O. Veblen, Geometry of four-component spinors, Proc. Nat. Acad. Sci. 19 (1933) 503 [INSPIRE].

[43] Yu.I. Manin, Holomorphic supergeometry and Yang-Mills superfields, J. Sov. Math. 30 (1985) 1927.

[44] Yu.I. Manin, Gauge field theory and complex geometry, Springer, Berlin Germany (1988) [INSPIRE].

[45] S.M. Kuzenko, J.-H. Park, G. Tartaglino-Mazzucchelli and R. Unge, Off-shell superconformal nonlinear $\sigma$-models in three dimensions, JHEP 01 (2011) 146 [arXiv: 1011.5727] [INSPIRE].

[46] P.S. Howe, Supergravity in superspace, Nucl. Phys. B 199 (1982) 309 [InSPIRE].

[47] S.M. Kuzenko, U. Lindström, M. Roček and G. Tartaglino-Mazzucchelli, On conformal supergravity and projective superspace, JHEP 08 (2009) 023 [arXiv:0905.0063] [INSPIRE].

[48] D. Butter, S.M. Kuzenko, J. Novak and G. Tartaglino-Mazzucchelli, Conformal supergravity in three dimensions: off-shell actions, JHEP 10 (2013) 073 [arXiv:1306.1205] [INSPIRE].

[49] S.M. Kuzenko, J. Novak and G. Tartaglino-Mazzucchelli, $N=6$ superconformal gravity in three dimensions from superspace, JHEP 01 (2014) 121 [arXiv: 1308.5552] [INSPIRE].

[50] D. Butter, B. de Wit, S.M. Kuzenko and I. Lodato, New higher-derivative invariants in $N=2$ supergravity and the Gauss-Bonnet term, JHEP 12 (2013) 062 [arXiv:1307.6546] [INSPIRE].

[51] B. Zupnik, Harmonic superpotentials and symmetries in gauge theories with eight supercharges, Nucl. Phys. B 554 (1999) 365 [Erratum ibid. B 644 (2002) 405] [hep-th/9902038] [INSPIRE].

[52] B. de Wit and S. Katmadas, Near-horizon analysis of $D=5$ BPS black holes and rings, JHEP 02 (2010) 056 [arXiv:0910.4907] [INSPIRE].

[53] L. Baulieu, M.P. Bellon and R. Grimm, BRS symmetry of supergravity in superspace and its projection to component formalism, Nucl. Phys. B 294 (1987) 279 [INSPIRE].

[54] P. Binetruy, G. Girardi and R. Grimm, Supergravity couplings: a geometric formulation, Phys. Rept. 343 (2001) 255 [hep-th/0005225] [INSPIRE]. 
[55] D. Butter and J. Novak, Component reduction in $N=2$ supergravity: the vector, tensor and vector-tensor multiplets, JHEP 05 (2012) 115 [arXiv:1201.5431] [INSPIRE].

[56] D. Butter, A new approach to curved projective superspace, arXiv:1406.6235 [INSPIRE].

[57] A.A. Rosly, Super Yang-Mills constraints as integrability conditions, in Proceedings of the International Seminar Group Theoretical Methods in Physics, Zvenigorod USSR (1982), vol. 1, M.A. Markov ed., Nauka, Moscow USSR (1983), pg. 263, english translation in Group Theoretical Methods in Physics, vol. 3, M.A. Markov, V.I. Man'ko and A.E. Shabad eds., Harwood Academic Publishers, London U.K. (1987), pg. 587.

[58] A. Galperin, E. Ivanov, S. Kalitsyn, V. Ogievetsky and E. Sokatchev, Unconstrained $N=2$ matter, Yang-Mills and supergravity theories in harmonic superspace, Class. Quant. Grav. 1 (1984) 469 [Corrigendum ibid. 2 (1985) 127] [INSPIRE].

[59] A.S. Galperin, E.A. Ivanov, V.I. Ogievetsky and E.S. Sokatchev, Harmonic superspace, Cambridge University Press, Cambridge U.K. (2001).

[60] F. Gonzalez-Rey, M. Roček, S. Wiles, U. Lindström and R. von Unge, Feynman rules in $N=2$ projective superspace: 1. Massless hypermultiplets, Nucl. Phys. B 516 (1998) 426 [hep-th/9710250] [INSPIRE].

[61] S.M. Kuzenko, U. Lindström, M. Roček and G. Tartaglino-Mazzucchelli, 4D N=2 supergravity and projective superspace, JHEP 09 (2008) 051 [arXiv: 0805.4683] [INSPIRE].

[62] J. Wess and J. Bagger, Supersymmetry and supergravity, Princeton University Press, Princeton U.S.A. (1992).

[63] S.J. Gates Jr., M.T. Grisaru, M. Roček and W. Siegel, Superspace or one thousand and one lessons in supersymmetry, Front. Phys. 58 (1983) 1, Benjamin/Cummings, Reading MA U.S.A. (1983) [hep-th/0108200] [INSPIRE].

[64] I.L. Buchbinder and S.M. Kuzenko, Ideas and methods of supersymmetry and supergravity or a walk through superspace, IOP, Bristol U.K. (1998).

[65] S.M. Kuzenko and G. Tartaglino-Mazzucchelli, $N=4$ supersymmetric Yang-Mills theories in $A d S_{3}$, JHEP 05 (2014) 018 [arXiv:1402.3961] [INSPIRE].

[66] B.M. Zupnik, The action of the supersymmetric $N=2$ gauge theory in harmonic superspace, Phys. Lett. B 183 (1987) 175 [INSPIRE].

[67] L. Mezincescu, On the superfield formulation of $O(2)$ supersymmetry, Dubna preprint JINR-P2-12572, USSR (1979) [INSPIRE].

[68] P.S. Howe, K.S. Stelle and P.K. Townsend, Supercurrents, Nucl. Phys. B 192 (1981) 332 [INSPIRE].

[69] D. Butter and S.M. Kuzenko, New higher-derivative couplings in $4 D N=2$ supergravity, JHEP 03 (2011) 047 [arXiv:1012.5153] [INSPIRE].

[70] D. Butter, S.M. Kuzenko and J. Novak, The linear multiplet and ectoplasm, JHEP 09 (2012) 131 [arXiv:1205.6981] [INSPIRE].

[71] L. Castellani, R. D'Auria and P. Fre, Supergravity and superstrings: a geometric perspective. Vol. 2: supergravity, World Scientific, Singapore (1991), pg. 680.

[72] M.F. Hasler, The three form multiplet in $N=2$ superspace, Eur. Phys. J. C 1 (1998) 729 [hep-th/9606076] [INSPIRE]. 
[73] S.J. Gates Jr., Ectoplasm has no topology: the prelude, in Supersymmetries and quantum symmetries, J. Wess and E.A. Ivanov eds., Springer, Berlin Germany (1999), pg. 46 [hep-th/9709104] [INSPIRE].

[74] S.J. Gates Jr., M.T. Grisaru, M.E. Knutt-Wehlau and W. Siegel, Component actions from curved superspace: normal coordinates and ectoplasm, Phys. Lett. B 421 (1998) 203 [hep-th/9711151] [INSPIRE].

[75] S.M. Kuzenko and G. Tartaglino-Mazzucchelli, Conformal supergravities as Chern-Simons theories revisited, JHEP 03 (2013) 113 [arXiv: 1212.6852] [INSPIRE].

[76] S.M. Kuzenko and J. Novak, Supergravity-matter actions in three dimensions and Chern-Simons terms, JHEP 05 (2014) 093 [arXiv: 1401.2307] [INSPIRE].

[77] S.M. Kuzenko and J. Novak, On supersymmetric Chern-Simons-type theories in five dimensions, JHEP 02 (2014) 096 [arXiv: 1309.6803] [INSPIRE].

[78] S.M. Kuzenko, Five-dimensional supersymmetric Chern-Simons action as a hypermultiplet quantum correction, Phys. Lett. B 644 (2007) 88 [hep-th/0609078] [INSPIRE].

[79] J. Novak, Superform formulation for vector-tensor multiplets in conformal supergravity, JHEP 09 (2012) 060 [arXiv:1205.6881] [INSPIRE].

[80] J. Novak, Variant vector-tensor multiplets in supergravity: classification and component reduction, JHEP 03 (2013) 053 [arXiv: 1210.8325] [INSPIRE].

[81] B. de Wit, R. Philippe and A. Van Proeyen, The improved tensor multiplet in $N=2$ supergravity, Nucl. Phys. B 219 (1983) 143 [INSPIRE].

[82] U. Lindström and M. Roček, Scalar tensor duality and $N=1, N=2$ nonlinear $\sigma$-models, Nucl. Phys. B 222 (1983) 285 [InSPIRE].

[83] S.M. Kuzenko, On $N=2$ supergravity and projective superspace: dual formulations, Nucl. Phys. B 810 (2009) 135 [arXiv:0807.3381] [INSPIRE].

[84] S.M. Kuzenko and S. Theisen, Correlation functions of conserved currents in $N=2$ superconformal theory, Class. Quant. Grav. 17 (2000) 665 [hep-th/9907107] [INSPIRE].

[85] S.M. Kuzenko, J. Novak and G. Tartaglino-Mazzucchelli, Symmetries of curved superspace in five dimensions, JHEP 10 (2014) 175 [arXiv: 1406.0727] [INSPIRE].

[86] D. Butter and S.M. Kuzenko, $N=2$ AdS supergravity and supercurrents, JHEP 07 (2011) 081 [arXiv:1104.2153] [INSPIRE].

[87] H. Nishino and S. Rajpoot, Alternative $N=2$ supergravity in five-dimensions with singularities, Phys. Lett. B 502 (2001) 246 [hep-th/0011066] [INSPIRE].

[88] F. Coomans and M. Ozkan, An off-shell formulation for internally gauged $D=5, N=2$ supergravity from superconformal methods, JHEP 01 (2013) 099 [arXiv:1210.4704] [INSPIRE].

[89] M. Zucker, Supersymmetric brane world scenarios from off-shell supergravity, Phys. Rev. D 64 (2001) 024024 [hep-th/0009083] [INSPIRE].

[90] B. de Wit and F. Saueressig, Off-shell $N=2$ tensor supermultiplets, JHEP 09 (2006) 062 [hep-th/0606148] [INSPIRE].

[91] S.M. Kuzenko, U. Lindström and R. von Unge, New extended superconformal $\sigma$-models and quaternion Kähler manifolds, JHEP 09 (2009) 119 [arXiv:0906.4393] [INSPIRE]. 
[92] A. Swann, Hyper-Kähler and quaternionic Kähler geometry, Math. Ann. 289 (1991) 421.

[93] B. de Wit, M. Roček and S. Vandoren, Hypermultiplets, hyper-Kähler cones and quaternion Kähler geometry, JHEP 02 (2001) 039 [hep-th/0101161] [INSPIRE].

[94] D. Butter, S.M. Kuzenko and G. Tartaglino-Mazzucchelli, Nonlinear $\sigma$-models with AdS supersymmetry in three dimensions, JHEP 02 (2013) 121 [arXiv:1210.5906] [INSPIRE].

[95] D. Butter, Projective multiplets and hyper-Kähler cones in conformal supergravity, arXiv: 1410.3604 [INSPIRE].

[96] G. Tartaglino-Mazzucchelli, $2 D N=(4,4)$ superspace supergravity and bi-projective superfields, JHEP 04 (2010) 034 [arXiv:0911.2546] [INSPIRE].

[97] G. Tartaglino-Mazzucchelli, On $2 D N=(4,4)$ superspace supergravity, Phys. Part. Nucl. Lett. 8 (2011) 251 [arXiv:0912.5300] [INSPIRE].

[98] S.M. Kuzenko, U. Lindström and G. Tartaglino-Mazzucchelli, Off-shell supergravity-matter couplings in three dimensions, JHEP 03 (2011) 120 [arXiv:1101.4013] [INSPIRE].

[99] W.D. Linch III and G. Tartaglino-Mazzucchelli, Six-dimensional supergravity and projective superfields, JHEP 08 (2012) 075 [arXiv:1204.4195] [INSPIRE].

[100] S.M. Kuzenko, Projective superspace as a double punctured harmonic superspace, Int. J. Mod. Phys. A 14 (1999) 1737 [hep-th/9806147] [INSPIRE].

[101] A. Galperin, E.A. Ivanov, V. Ogievetsky and E. Sokatchev, Harmonic supergraphs: Green functions, Class. Quant. Grav. 2 (1985) 601 [InSPIRE].

[102] S.M. Kuzenko and I.N. McArthur, Effective action of $N=4$ super Yang-Mills: $N=2$ superspace approach, Phys. Lett. B 506 (2001) 140 [hep-th/0101127] [INSPIRE]. 